Spectral Analysis of the

Turbulent Mixing of Two Fluids 
This thesis was accepted by the Graduate College, University of Illinois at Urbana-Champaign, Illinois, in partial fulfillment of the requirements for the degree of Doctor of Philosophy. The text and illustrations are the independent work of the author and only the front matter has been edited by the CIC-1 staff to conform with Department of Energy and Los Alamos National Laboratory publication policies.

An Affirmative Action/Equal Opportunity Employer

This report was prepared as an account of work sponsored by an agency of the United States Government. Neither The Regents of the University of Califormia, the United States

Government nor any agency thereof, nor any of their employees, makes any warranty, express or implied, or assumes any legal liability or responsibility for the accuracy, completeness, or usefulness of any information, apparatus, product, or process disclosed, or represents that its use would not infringe privately owned rights. Reference herein to any specific commercial product, process, or service by trade name, trademark, manufacturer, or otherwise, does not necessarily constitute or imply its endorsement, recommendation, or favoring by The Regents of the University of California, the United States Government, or any agency thereof. The views and opinions of authors expressed herein do not necessarily state or reflect those of The Regents of the University of California, the United States Government, or any agency thereof. The Los Alamos National Laboratory strongly supports academic freedom and a researcher's right to publish; therefore, the Laboratory as an institution does not endorse the viewpoint of a publication or guarantee its technical correctness. 
Spectral Analysis of the

Turbulent Mixing of Two Fluids

Michael James Steinkamp 


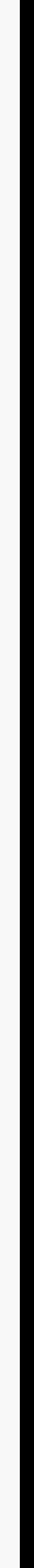




\title{
SPECTRAL ANALYSIS OF THE TURBULENT MIXING OF TWO FLUIDS
}

by

\author{
Michael James Steinkamp
}

\section{ABSTRACT}

We describe a spectral approach to the investigation of fluid instability, generalized turbulence, and the interpenetration of fluids across an interface. The technique also applies to a single fluid with large variations in density. Departures of fluctuating velocity components from the local mean are far subsonic, but the mean Mach number can be large. Validity of the description is demonstrated by comparisons with experiments on turbulent mixing due to the late stages of Rayleigh-Taylor instability, when the dynamics become approximately self-similar in response to a constant body force. Generic forms for anisotropic spectral structure are described and used as a basis for deriving spectrally integrated moment equations that can be incorporated into computer codes for scientific and engineering analyses. 


\section{Table of Contents}

List of Tables. ..viii

List of Figures ix

Nomenclature. xviii

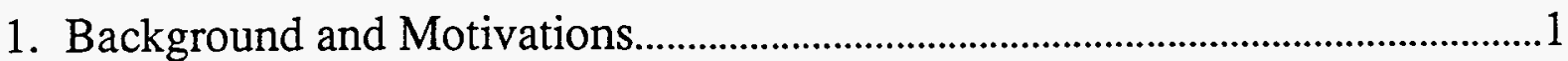

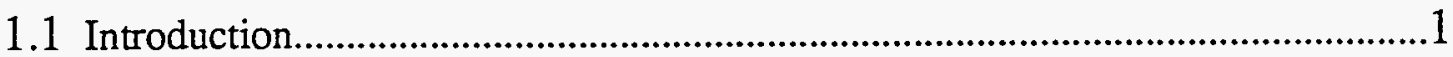

1.2 Research summary ..........................................................................................

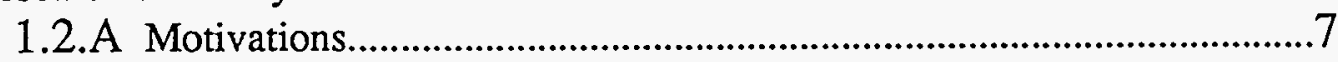

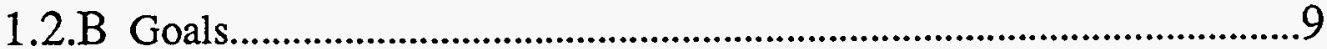

1.2.C Strategy ............................................................................................. 10

1.2.D Contributions.......................................................................................11

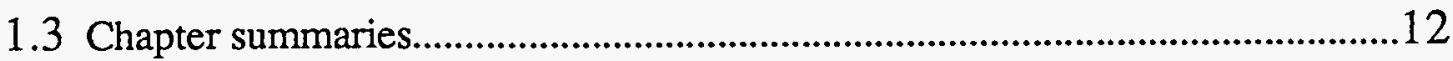

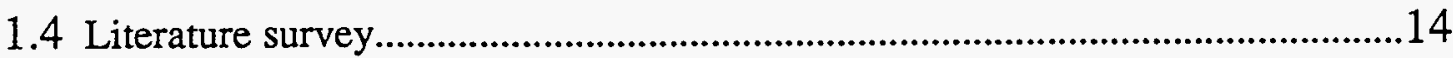

1.4.A Two-fluid turbulent mixing due to the Rayleigh-Taylor instability ..14

1.4.B Turbulence transport modeling..............................................................24

2. Configurational Definition of the Transported Variables Describing the

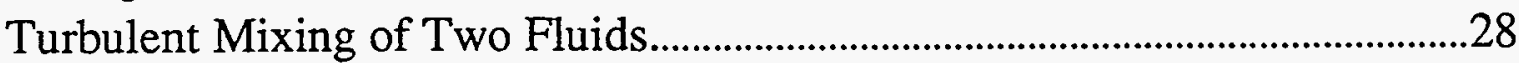

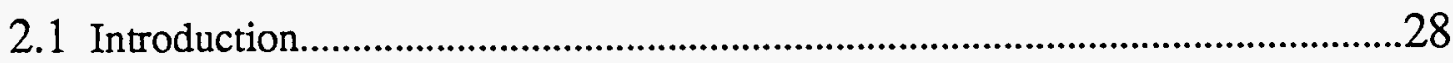

2.2 Description of the flow field variables.........................................................29

2.3 Summary of the flow field variables....................................................................38

3. Single-Point Turbulence Transport Modeling..........................................................39

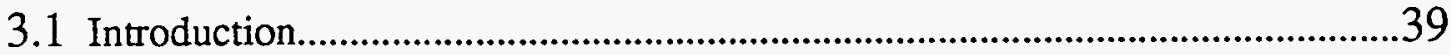

3.2 Single-point turbulence transport equations......................................................

3.3 Numerical results for the single-point turbulence transport approach................50

3.4 Summary of the single-point turbulence transport approach................................57

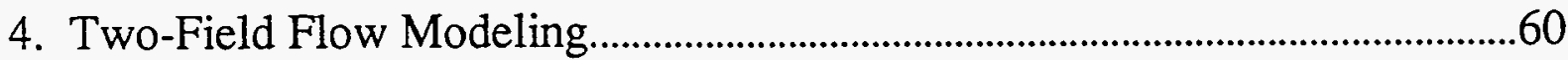

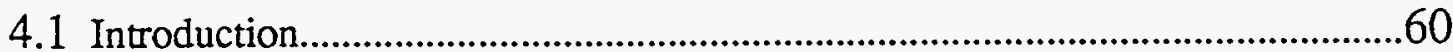

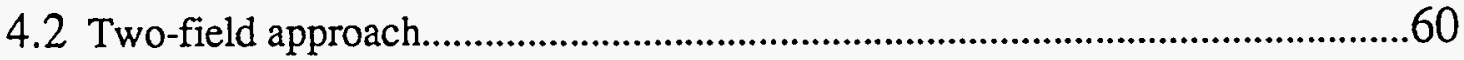

4.3 Numerical results for the two-field approach...................................................65

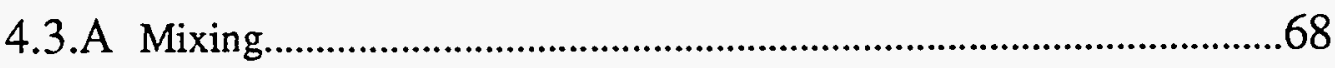

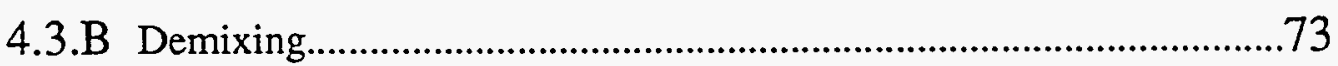

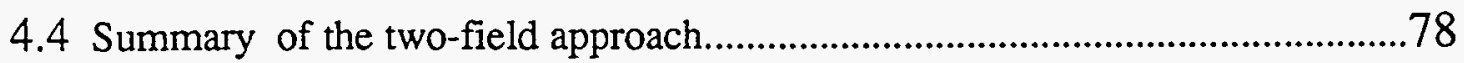

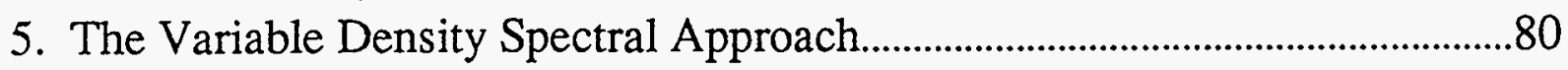

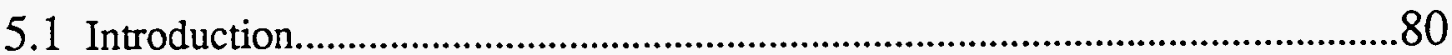

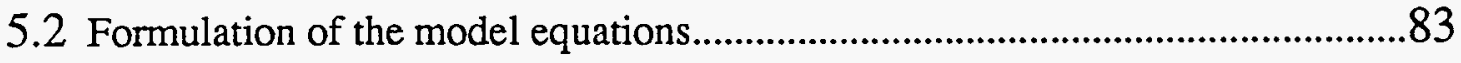

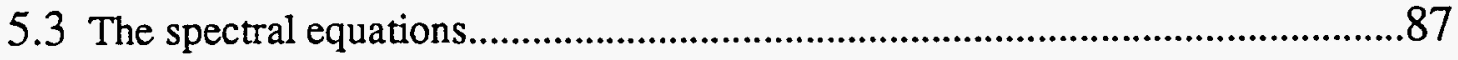


6. Behavior of the Model Terms in both Physical Space

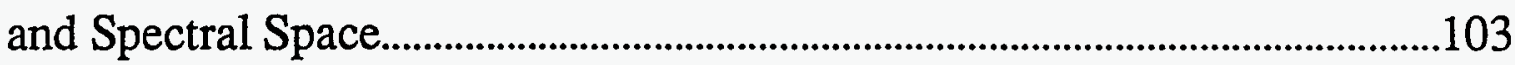

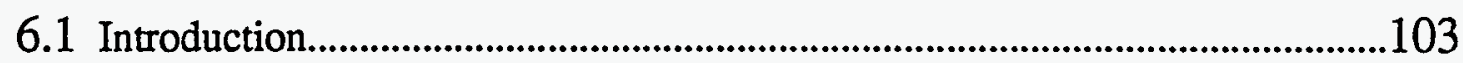

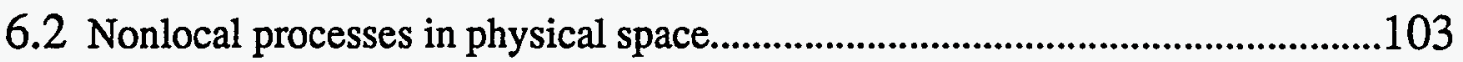

6.3 Spectral behavior of the model terms................................................................110

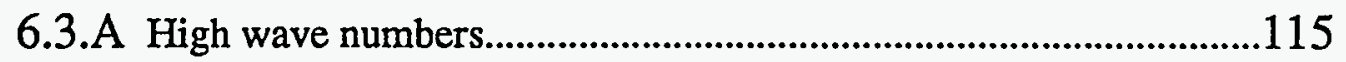

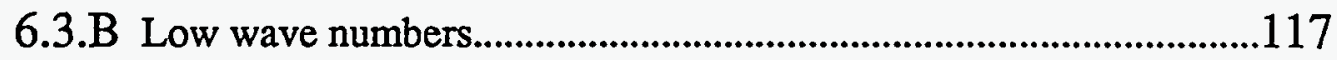

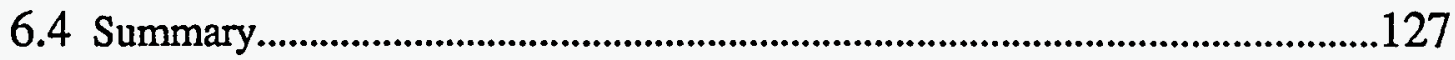

7. Numerical Results for the Spectral Turbulence Transport Equations............128

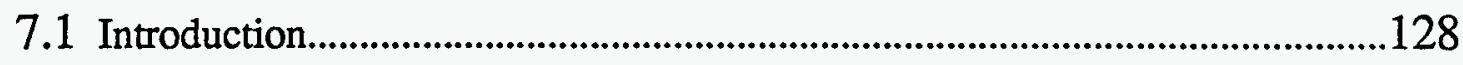

7.2 Mixing due to an acceleration...........................................................................129

7.3 Numerical results for a rapid transient................................................................

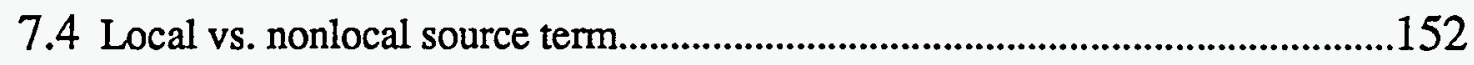

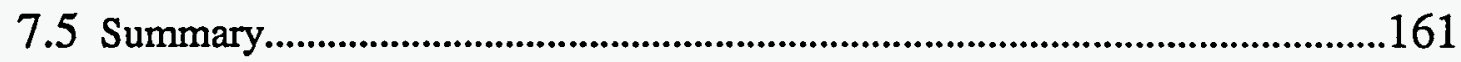

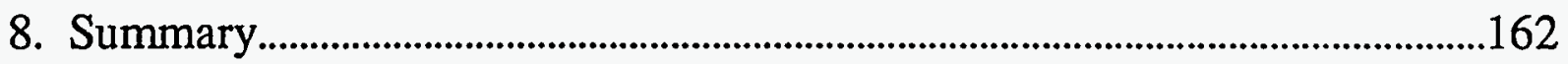

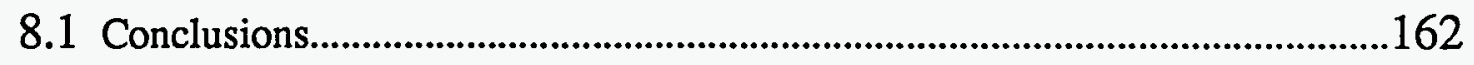

8.2 Future research directions.....................................................................................165

Appendix A. Conservation of Energy for Single-Point Transport Equations..................................................168

Appendix B. Closure for the Single-Point b-Equation...........................................174

Appendix C. Categorizing Two-Fluid Interpenetration..........................................178

Appendix D. Self-Similar Form Functions...............................................................182

Appendix E. Moments of the Spectral Equations...................................................187

Appendix F. Numerical Schemes............................................................................195

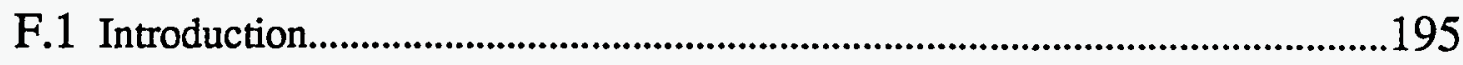

F.2 Solution procedure for the single-point turbulence transport equations...........196

F.3 Solution procedure for the two-field transport equations....................................204

F.4 Solution procedure for the spectral turbulence transport equations..................213

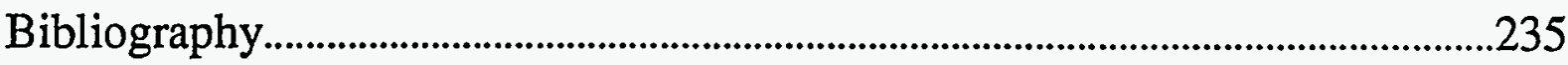

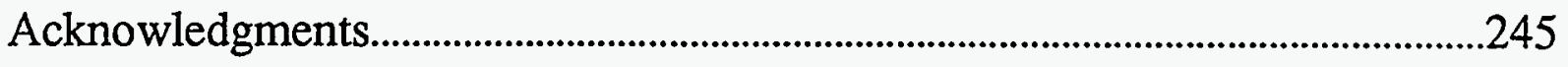




\section{List of Tables}

\begin{tabular}{|l|c|r|}
\hline Table & Title & Page \\
\hline 7.3.1. & $\begin{array}{l}\text { Power law behavior for both the low and high wave } \\
\text { numbers for the transported spectral variables. }\end{array}$ & 152 \\
\hline 7.4.1. & $\begin{array}{c}\text { Self-similar coefficient, } \alpha, \text { and the asymmetry of the } \\
\text { TMZ for the two numerical runs and experiments. }\end{array}$ & 153 \\
\hline
\end{tabular}




\section{List of Figures}

\begin{tabular}{|c|c|c|}
\hline Figure & Title & Page \\
\hline 1.1.1. & $\begin{array}{l}\text { Flow chart defining the ancestry of the our spectral } \\
\text { transport model. }\end{array}$ & 4 \\
\hline 1.1.2. & $\begin{array}{l}\text { Diagram showing the "family tree" for turbulence } \\
\text { models. }\end{array}$ & 6 \\
\hline 2.2.1. & $\begin{array}{l}\text { Idealized schematic of an interface between two } \\
\text { interpenetrating fluids (top) with the corresponding } \\
\text { density profile (bottom) found in the thin horizontal strip } \\
\text { through the mixing layer. }\end{array}$ & 33 \\
\hline 3.3.1. & Density profile across TMZ at $t=30$. & 54 \\
\hline 4.3.1. & $\begin{array}{l}\text { Examples of various two-field variables plotted across } \\
\text { the TMZ throughout the mixing process of the Rayleigh- } \\
\text { Taylor instability. Plots (a), (c) and (d) illustrate the time } \\
\text { evolution of the variables by plotting a profile every } 10 \\
\text { time units from a time of } 0 \text { to } 50 \text {. Plots (b) and (e) show } \\
\text { the variables at a time of } 50 \text {. Plot (f) illustrates the effects } \\
\text { of cell size on the physical instability that is becoming } \\
\text { significant by a time of } 55 .\end{array}$ & 70 \\
\hline 4.3.2. & $\begin{array}{l}\text { Comparison to experiment of the volume fraction of the } \\
\text { top fluid for a mixing calculation. The solid line is the } \\
\text { numerical results and the symbols are data from Fig. } 25 \\
\text { (exp. \#94) of Smeeton \& Youngs (1987). }\end{array}$ & 72 \\
\hline
\end{tabular}




\begin{tabular}{|c|c|c|}
\hline Figure & Title & Page \\
\hline 4.3.3. & $\begin{array}{l}\text { Plots (a) and (b) illustrate the evolution of the } \\
\text { penetration depth for the bubbles, } h_{1} \text {, spikes, } h_{2} \text {, the } \\
\text { overall width of the TMZ, and the scale that is calculated } \\
\text { for these equations. Note, the profiles grow quadratically } \\
\text { in time in plot (a) and linearly in X in plot (b). Plot (c) } \\
\text { shows the spike to bubble ratio, which has leveled off to } \\
1.25 \text {, and plot (d) shows the self-similarity coefficient, } \alpha \text {, } \\
\text { which is the value of the slope of the } h_{1} \text { profile in plot (b). }\end{array}$ & 72 \\
\hline 4.3.4. & $\begin{array}{l}\text { Schematic of the computational grid. Pure fluid \#1 is } \\
\text { below the TMZ, which is comprised of both fluids. The } \\
\text { lower edge of the TMZ is located in cell \#j. }\end{array}$ & 74 \\
\hline 4.3.5. & $\begin{array}{l}\text { Plots (a), (c), and (e) show the variable profiles through } \\
\text { a time of } 30 \text {, which corresponds to the time at which the } \\
\text { acceleration is reversed, and plots (b), (d), and (f) } \\
\text { illustrate the demixing that results from the acceleration } \\
\text { reversal. }\end{array}$ & 75 \\
\hline 4.3.6. & $\begin{array}{l}\text { Mass-fluxing velocity profiles for (a) times of } 10,20 \text {, } \\
\text { and } 30 \text {, to show mixing, (b) times of every } 0.5 \text { from } 30 \\
\text { through } 35 \text {, to show the reversal due to the acceleration } \\
\text { reversal at } t=30 \text {, and (c) times of every } 5 \text { from } 35 \\
\text { through } 50 \text {, to show the decrease in velocity due to the } \\
\text { diminishing TMZ. }\end{array}$ & 76 \\
\hline
\end{tabular}




\begin{tabular}{|c|c|c|}
\hline Figure & Title & Page \\
\hline 4.3 .7 . & $\begin{array}{l}\text { Time evolution of the (a) width of the TMZ, amplitude } \\
\text { of the scale of the flow, and the depth of penetration of } \\
\text { the spikes, } \mathrm{h}_{2} \text {, and the bubbles, } \mathrm{h}_{1},(\mathrm{~b}) \text { spike to bubble } \\
\text { ratio, and (c) self-similarity coefficient for the entire } \\
\text { mixing and demixing process. }\end{array}$ & 77 \\
\hline 5.2.1. & Flow chart describing steps of the spectral formulation & 84 \\
\hline 5.2.2. & $\begin{array}{l}\text { Flow chart describing all of the potential uses of a } \\
\text { spectral formulation. }\end{array}$ & 85 \\
\hline 6.2.1. & $\begin{array}{l}\text { Schematic to demonstrate the growth and saturation of } \\
\text { initial perturbations of the Rayleigh-Taylor instability. }\end{array}$ & 109 \\
\hline 6.3.1. & $\begin{array}{l}\text { Schematic of the local source term across the TMZ as a } \\
\text { function of } y \text { and } k \text {. The maximum source corresponds to } \\
\text { the wave number } k_{1} \text { where the spectrum of } a(k) \text { attains an } \\
\text { extremum. }\end{array}$ & 119 \\
\hline 6.3.2. & $\begin{array}{l}\text { Schematic representation of the shape of the } Q \text { function. } \\
\text { Notice how the base in the } y \text { direction becomes more } \\
\text { narrow for larger values of } k \text {. The area contained by each } \\
\text { curve is the same due to normalization. }\end{array}$ & 121 \\
\hline 6.3.3. & $\begin{array}{l}\text { Schematic representation of the effect of the } Q \text {-function } \\
\text { on the local source, } S_{\mathrm{ij}}^{\mathrm{L}} \text {, to } R_{\mathrm{ij}}(k) \text { for a generic wave } \\
\text { numbers. }\end{array}$ & 121 \\
\hline 6.3.4. & $\begin{array}{l}\text { Schematic to represent the different structures through } \\
\text { the TMZ of the nonlocal source term to } \partial R_{i j} / \partial t \text { due to } \\
\text { the dependence of the nonlocality on wave number } k \text {. }\end{array}$ & 124 \\
\hline
\end{tabular}




\begin{tabular}{|c|c|c|}
\hline Figure & Title & Page \\
\hline 6.3.5. & $\begin{array}{l}\text { Schematic representation of the spectral modifications to } \\
\text { the time rate of change of the Reynolds stress tensor due } \\
\text { to a nonlocal source term for: (a) the edge of the TMZ } \\
\text { and (b) the center of the TMZ. }\end{array}$ & 125 \\
\hline 7.2.1. & $\begin{array}{l}\text { Spectra at times } t=10,20,30,40,50 \text {, and } 60 \text {, at the } \\
\text { position of the initial fluid interface: (a) the net mass-flux } \\
\text { velocity, } a,(b) \text { the specific volume-density correlation, } b \text {, } \\
\text { (c) the contraction of the Reynolds stress tensor, } R_{n n} \text {, and } \\
\text { (d) the } R_{\mathrm{yy}} \text { component of the Reynolds stress tensor. }\end{array}$ & 131 \\
\hline 7.2.2. & $\begin{array}{l}\text { Normalized spectra for } t=30,35,40,45,50,55,60 \text {, and } \\
65 \text {, at the position of the initial fluid interface: (a) the net } \\
\text { mass-flux velocity, a, (b) the specific volume-density } \\
\text { correlation, } b \text {, (c) the contraction of the Reynolds stress } \\
\text { tensor, } R_{n n} \text {, and (d) the } R_{y y} \text { component of the Reynolds } \\
\text { stress tensor. }\end{array}$ & 134 \\
\hline 7.2.3. & $\begin{array}{l}\text { Profiles of the power law exponent across the TMZ at } \\
t=65 \text { for (a) the low wave numbers, and (b) the high } \\
\text { wave numbers. }\end{array}$ & 134 \\
\hline 7.2.4. & $\begin{array}{l}\text { Normalized spectra for } t=30,35,40,45,50,55,60 \text {, } \\
\text { and } 65 \text {, a distance of } 50 \text { units of length below the } \\
\text { centerline of the TMZ: (a) the net mass-flux velocity, } \\
\text { (b) the specific volume-density correlation, (c) the } \\
\text { contraction of the Reynolds stress tensor, and (d) the } \mathrm{R}_{\mathrm{yy}} \\
\text { component of the Reynolds stress tensor. }\end{array}$ & 135 \\
\hline
\end{tabular}




\begin{tabular}{|l|l|r|}
\hline Figure & \multicolumn{1}{|c|}{ Title } & Page \\
\hline 7.2.5. & $\begin{array}{l}\text { Comparison of normalized spectra at } \mathrm{y}=0 \text { and } \mathrm{y}=-50 \\
\text { for } \mathrm{t}=65 \text { for the variables: (a) the net mass-flux velocity, } \\
\text { (b) the specific volume-density correlation, (c) the } \\
\text { contraction of the Reynolds stress tensor, and (d) the } \mathrm{R}_{\mathrm{yy}} \\
\text { component of the Reynolds stress tensor. }\end{array}$ & \\
\hline 7.2.6. & $\begin{array}{l}\text { Comparison of normalized spectra at } \mathrm{y}=0 \text { and } \mathrm{y}=50 \\
\text { for } \mathrm{t}=65 \text { for the variables: (a) the net mass-flux velocity, } \\
\text { (b) the specific volume-density correlation, (c) the } \\
\text { contraction of the Reynolds stress tensor, and (d) the } \mathrm{R}_{\mathrm{yy}} \\
\text { component of the Reynolds stress tensor. }\end{array}$ & \\
\hline 7.2.7. & $\begin{array}{l}\text { Profiles of the length scales across the TMZ for } \mathrm{t}=5, \\
15,25,35,45,55, \text { and } 65: \text { (a) the net mass-flux velocity, } \\
\text { (b) the specific volume-density correlation, (c) the } \\
\text { contraction of the Reynolds stress tensor, and (d) the } \mathrm{R}_{\mathrm{yy}} \\
\text { component of the Reynolds stress tensor. }\end{array}$ & \\
\hline 7.2 .8$. & $\begin{array}{l}\text { Profiles of spectrally integrated variables across the } \\
\text { TMZ for } \mathrm{t}=5,15,25,35,45,55, \text { and } 65: \text { (a) the net. } \\
\text { mass-flux velocity, (b) the specific volume-density } \\
\text { correlation, (c) the contraction of the Reynolds stress } \\
\text { tensor, and (d) the } \mathrm{R}_{\mathrm{yy}} \text { component of the Reynolds stress } \\
\text { tensor. }\end{array}$ & \\
\hline
\end{tabular}




\begin{tabular}{|c|c|c|}
\hline Figure & Title & Page \\
\hline 7.2.9. & $\begin{array}{l}\text { (a) Profiles of density across the TMZ for } t=0,5,15 \text {, } \\
25,35,45,55 \text {, and } 65 \text {. (b) Mass and volume fractions of } \\
\text { the heavy fluid across the TMZ at } t=65 \text {. (c) Comparison } \\
\text { of numerical results with AWE experimental data } \\
\text { [Smeeton and Youngs, } 1987 \text { (Fig. } 24 \text { and 25)]. } \\
\text { (d) Comparison of numerical results with Chelyabinsk-70 } \\
\text { experimental data [Kucherenko et al. } 1991 \text { (Fig. 11)] }\end{array}$ & 140 \\
\hline 7.2.10. & $\begin{array}{l}\text { Spikes, } h_{2} \text {, and the bubbles, } h_{1}, \text { (a) as functions of time } \\
\text { and (b) as functions of the similarity variable, } X \text {. }\end{array}$ & 141 \\
\hline 7.2.11 & $\begin{array}{l}\text { Comparison of the trace of the Reynolds stress tensor } \\
\text { with } R_{y y}(a) \text { at the centerline at } t=65 \text { and (b) in spectrally } \\
\text { integrated form across the TMZ. }\end{array}$ & 143 \\
\hline 7.2.12. & $\begin{array}{l}\text { Profile of } b \text { across the TMZ for the case of a spectrally } \\
\text { unblocked calculation and a spectrally blocked } \\
\text { calculation compared with the configurational calculation } \\
\text { of } b \text {, at } t=65 \text {. }\end{array}$ & 145 \\
\hline 7.3.1. & $\begin{array}{l}\text { Spectral contributions of the dominant sources and sinks } \\
\text { to the a equation for: (a) mixing }(g=-1) \text {, and } \\
\text { (b) demixing, immediately after acceleration reversal } \\
(\mathrm{g}=1) \text {. }\end{array}$ & 147 \\
\hline
\end{tabular}




\begin{tabular}{|c|c|c|}
\hline Figure & Title & Page \\
\hline 7.3.2. & $\begin{array}{l}\text { Spectra at times } t=65 \text { and } 80 \text {, at the position of the } \\
\text { initial fluid interface, for: (a) the net mass-flux velocity, a } \\
\text { (including plots for every } \Delta t=1.5 \text { ), (b) the specific } \\
\text { volume-density correlation, } b \text {, (c) the contraction of the } \\
\text { Reynolds stress tensor, } R_{n n} \text {, and (d) the } R_{y y} \text { component of } \\
\text { the Reynolds stress tensor. }\end{array}$ & 149 \\
\hline 7.3.3. & $\begin{array}{l}\text { Normalized spectra for } t=65 \text { and } 80 \text {, at the position of } \\
\text { the initial fluid interface, for: (a) the net mass-flux } \\
\text { velocity, } a \text {, (b) the specific volume-density correlation, } b \text {, } \\
\text { (c) the contraction of the Reynolds stress tensor, } R_{n n} \text {, and, } \\
\text { (d) the } R_{\mathrm{yy}} \text { component of the Reynolds stress tensor. }\end{array}$ & 150 \\
\hline 7.3.4. & $\begin{array}{l}\text { Profiles of spectrally integrated variables across the } \\
\text { TMZ for } t=65 \text { and } 80 \text { of (a) the net mass-flux velocity, a } \\
\text { (including plots for every } \Delta t=1.5 \text { ), (b) the specific } \\
\text { volume-density correlation, } b \text {, (c) the contraction of the } \\
\text { Reynolds stress tensor, } R_{n n} \text {, and (d) the } R_{y y} \text { component of } \\
\text { the Reynolds stress tensor. }\end{array}$ & 151 \\
\hline 7.3.5. & Profiles of density across the TMZ for $t=65$ and 80 . & 151 \\
\hline 7.4.1. & $\begin{array}{l}\text { Comparison of the density across the TMZ for a local } \\
\text { source to } \mathrm{R}_{\mathrm{ij}} \text { and a nonlocal source to } \mathrm{R}_{\mathrm{ij}} \text {. }\end{array}$ & 154 \\
\hline
\end{tabular}




\begin{tabular}{|c|c|c|}
\hline Figure & Title & Page \\
\hline 7.4.2. & $\begin{array}{l}\text { Comparison of the low wave number power law } \\
\text { behavior of } k \text { at } t=65 \text { for a local and nonlocal run for: } \\
\text { (a) the net mass-flux velocity, a, (b) the specific volume- } \\
\text { density correlation, } b,(c) \text { the contraction of the Reynolds } \\
\text { stress tensor, } R_{n n} \text {, and, (d) the } R_{y y} \text { component of the } \\
\text { Reynolds stress tensor. }\end{array}$ & 154 \\
\hline 7.4.3. & $\begin{array}{l}\text { Comparison of the high wave number power law } \\
\text { behavior of } k \text { at } t=65 \text { for a local and nonlocal run for: } \\
\text { (a) the net mass-flux velocity, a, (b) the specific volume- } \\
\text { density correlation, } b \text {, (c) the contraction of the Reynolds } \\
\text { stress tensor, } R_{n n} \text {, and, (d) the } R_{y y} \text { component of the } \\
\text { Reynolds stress tensor. }\end{array}$ & 155 \\
\hline 7.4.4. & $\begin{array}{l}\text { Normalized spectra for } t=30,35,40,45,50,55,60 \text {, and } \\
65 \text {, a distance of } 50 \text { units of length below the centerline of } \\
\text { the TMZ for: (a) the net mass-flux velocity, a, (b) the } \\
\text { specific volume-density correlation, } b \text {, (c) the contraction } \\
\text { of the Reynolds stress tensor, } R_{n n} \text {, and, (d) the } R_{y y} \\
\text { component of the Reynolds stress tensor. }\end{array}$ & 156 \\
\hline 7.4.5. & $\begin{array}{l}\text { Comparison of the profiles of the length scales across } \\
\text { the TMZ for a local and nonlocal run at } t=65 \text { for: } \\
\text { (a) the net mass-flux velocity, } a \text {, (b) the specific volume- } \\
\text { density correlation, } b,(c) \text { the contraction of the Reynolds } \\
\text { stress tensor, } R_{n n} \text {, and, (d) the } R_{y y} \text { component of the } \\
\text { Reynolds stress tensor. }\end{array}$ & 158 \\
\hline
\end{tabular}




\begin{tabular}{|c|c|c|}
\hline Figure & Title & Page \\
\hline 7.4.6. & $\begin{array}{l}\text { Experimental data from Chelyabinsk-70 (Kucherenko } \\
\text { et al. 1991) for: (a) concentration profile through TMZ, } \\
\text { (b) bubble and spike penetration depth, (c) asymmetry of } \\
\text { spike to bubble ratio, and (d) comparison of numerical } \\
\text { results to experiments (Kucherenko et al. 1991; Smeeton } \\
\text { and Youngs, 1987). }\end{array}$ & 159 \\
\hline 7.4.7. & $\begin{array}{l}\text { Comparison of the low wave number power law } \\
\text { behavior of } k \text { at } t=65 \text { for two different values of } C_{f b} \text { for: } \\
\text { (a) the net mass-flux velocity, a, (b) the specific volume- } \\
\text { density correlation, } b \text {, (c) the contraction of the Reynolds } \\
\text { stress tensor, } R_{n n} \text {, and, (d) the } R_{y y} \text { component of the } \\
\text { Reynolds stress tensor. }\end{array}$ & 160 \\
\hline A.1. & Schematic of an idealized TMZ & 169 \\
\hline C.1. & $\begin{array}{l}\text { Schematic of a two-dimensional box filled with sandy } \\
\text { liquid on the right side and pure liquid on the left side } \\
\text { separated by a vertical partition. }\end{array}$ & 179 \\
\hline C.2. & $\begin{array}{l}\text { Schematic of a two-dimensional box filled with pure } \\
\text { liquid on the left side of the partition and sandy liquid on } \\
\text { the right side of the partition. Gravity produces a pressure } \\
\text { gradient across the interface such that when the partition } \\
\text { is removed the highest pressure will be in the pure liquid. }\end{array}$ & 180 \\
\hline F.1. & $\begin{array}{l}\text { Logic scheme for the numerical solution of the BHR } \\
\text { single-point turbulence transport model. }\end{array}$ & 197 \\
\hline F.2. & $\begin{array}{l}\text { Logic scheme for the numerical solution of the two-field } \\
\text { transport model. }\end{array}$ & 205 \\
\hline F.3. & $\begin{array}{l}\text { Logic schematic for the numerical solution of the two- } \\
\text { point spectral transport equations. }\end{array}$ & 215 \\
\hline
\end{tabular}




\section{Nomenclature}

A Amplitude of perturbations (Rayleigh-Taylor linearized analysis).

$\mathbf{a}, \mathrm{a}_{\mathbf{i}} \quad$ Velocity of the net mass flux.

At Dimensionless Atwood number.

b Density-specific volume correlation.

B Density-density correlation.

$\mathrm{C}_{\mathrm{d}}$ Diffusion constant for spectral model pertaining to triple correlations.

CDR Diffusion constant for the single-point (BHR) model.

$\mathrm{C}_{\mathrm{db}} \quad$ Constant for molecuar diffusion term in spectral b-equation.

$\mathrm{C}_{\mathrm{fb}}$ Model constant pertaining to mean flow gradient coupling to eddy distortion.

$c_{1} c_{2}$ Mass fractions of fluid \#1 and fluid \#2 respectively.

$\mathrm{C}_{1} \mathrm{C}_{2}$ Model constants pertaining to the triple correlations goverining Fourier space cascade.

$\mathrm{C}_{1 \mathrm{R}} \mathrm{C}_{2 \mathrm{R}} \quad \mathrm{BHR}$ model constants for the pressure-velocity gradient correlations.

$\mathrm{C}_{\mathrm{m}} \quad$ Model constant pertaining to the slow part of the fluctuating pressure-strain correlation.

$\mathrm{C}_{\mathrm{rp} 1} \mathrm{C}_{\mathrm{rp} 2}$

Model constants pertaining to interfluid drag.

$\mathrm{C}_{\mathrm{S} 1} \mathrm{C}_{\mathrm{S} 2} \mathrm{C}_{\mathrm{S} 3} \mathrm{C}_{\mathrm{S} 4} \quad$ Model constants for the $\mathrm{S}$-equation in the single-point BHR model.

$\mathrm{C}_{1 \varepsilon} \mathrm{C}_{2 \varepsilon} \mathrm{C}_{3 \varepsilon} \mathrm{C}_{\mathrm{D \varepsilon}} \quad$ Model constants for the e-equation in the single-point BHR model.

$\mathrm{C}_{1 \mathrm{a}} \mathrm{C}_{2 \mathrm{a}} \mathrm{CDa}_{\mathrm{Da}} \quad$ Model constants for the a-equation in the single-point BHR model.

D Diffusion coefficient for molecular mixing of fluid species.

$\mathrm{d} \Omega_{\mathrm{k}} \quad$ Differential area of sphere in Fourier wavenumber space.

E Turbulent kinetic energy spectrum.

F Force vector.

$F_{\mathrm{a}} \quad$ Self-similar spectral shape function for $\mathbf{a}$.

$F_{b} \quad$ Self-similar spectral shape function for $b$. 
$F_{n n} \quad$ Self-similar spectral shape function for $R_{n n}$.

$F_{y y} \quad$ Self-similar spectral shape function for $R_{y y}$.

g Acceleration.

go Acceleration due to gravity $\left(980 \mathrm{~cm} / \mathrm{sec}^{2}\right)$.

$h_{1}$ Depth of bubble penetration into heavier fluid in a two-fluid mixing layer.

$\mathrm{h}_{2}$ Depth of spike penetration into lighter fluid in a two-fluid mixing layer.

$\mathrm{K} \quad$ Turbulent kinetic energy per unit mass.

$\mathrm{K}_{0} \quad$ Fraction of ordered part of $\mathrm{K}$ (two-phase flow model).

$\mathrm{K}_{\mathrm{D}} \quad$ Momentum exchange term in the Navier-Stokes equations.

$\mathrm{K}_{\mathrm{D}}^{*}$ Drag term in the two-phase flow a-equation.

$\mathrm{K}^{\mathfrak{c}} \quad$ Configurational expression for the ordered turbulent kinetic energy per unit mass.

$\mathrm{K}_{\text {diss }}$ Disordered part of theturbulent kinetic energy per unit mass.

k Fourier wavenumber vector.

k Fourier wavenumber magnitude (a scalar).

$k_{\max }$ Value of wavenumber $k$ at which the spectrum attains an extremum.

k0 Reference wavenumber used to nondimensionalize the spectral equations.

$\mathrm{k}_{1} \quad$ Wavenumber at which $\mathrm{a}$ attains an extremum.

$\mathrm{L}_{\mathrm{a}} \quad$ Single-point length scale for single-point $\mathbf{a}$.

$L_{b} \quad$ Single-point length scale for single-point $b$.

$L_{R} \quad$ Single-point length scale for single-point $R_{i j}$.

M Total mass of fluids in a given volume.

$\mathrm{m}_{1} \mathrm{~m}_{2} \quad$ Mass of fluids $\# 1$ and $\# 2$ in a differential volume element.

$\mathrm{n} \quad$ Power law for initial conditions of $\mathrm{b}$ spectrum (at low wavenumbers).

p Instantaneous pressure.

$\overline{\mathrm{p}} \quad$ Average pressure.

$\mathrm{p}^{\prime} \quad$ Fluctuating pressure.

Q Spreading function for nonlocal model source term to $R_{i j}$ transport equation. 
r Separation vector for two-point spectral variables defined as $\mathbf{x}_{1}-\mathbf{x}_{2}$.

$R_{\mathrm{ij}} \quad$ Reynolds stress tensor.

$R_{i j}^{c} \quad$ Configurational expression for the Reynolds stress tensor.

$\mathrm{R}_{\mathrm{ijk}} \quad$ Triple velocity correlation.

$S \quad$ Single-point length scale of turbulence.

$S_{i j}^{L} \quad$ Local model source term to $R_{i j}$ transport equation.

$\mathrm{S}_{\mathrm{ij}}^{\mathrm{NL}} \quad$ Nonlocal model source term to $\mathrm{R}_{\mathrm{ij}}$ transport equation.

I Time.

u Instantaneous velocity.

$\tilde{\mathbf{u}}, \tilde{u}_{\mathrm{i}} \quad$ Mass-weighted ensemble averaged velocity (via Favre decomposition).

$\mathbf{u}^{\prime \prime}, \mathrm{u}_{\mathrm{i}}^{\prime \prime}$ Fluctuations in the mass-weighted velocity, $\tilde{\mathbf{u}}$.

$\overline{\mathbf{u}}, \bar{u}_{\mathrm{i}} \quad$ Volume-weighted average velocity (via Reynolds decomposition).

$\mathbf{u}^{\prime}, \mathrm{u}_{\mathrm{i}}^{\prime}$ Fluctuations in the volume-weighted velocity, $\overline{\mathbf{u}}$.

$\mathbf{u}_{1} \quad$ Average velocity of fluid \#1 in a two-field flow.

$\mathbf{u}_{2}$ Average velnsity of fluid \#2 in a two-field flow.

W Width of turbulent mixing zone.

X Self-similar variable for the turbulent mixing layer.

$\mathbf{x}_{1}, \mathbf{x}_{2}$ Spatial positions of variables for the two-point spectral turbulence model.

$\mathbf{x}$ Position vector for two-point spectral variables defined as $\left(\mathbf{x}_{1}+\mathbf{x}_{2}\right) / 2$.

y Spatial independent variable.

z Nondimensional independent variable $\left(\ln \left(\mathrm{k} / \mathrm{k}_{0}\right)\right)$ of spectral equations.

Greek symbols

$\alpha \quad$ Self similar coefficient for evolution of bubble interpenetration depth.

$\alpha_{i} \quad$ Instantaneous volume fraction of the $i$-th fluid.

$\beta \quad$ Constant to regulate the growth of the length scale in the two-fluid model.

$\Delta \quad$ Change. 
$\delta \quad$ Value of volume fraction at edge of $\mathrm{TMZ}$ at which the Chelyabinsk-70 experimental measurements are taken.

$\delta$ V Differential volume element.

$\delta \mathrm{x} \quad$ Computational grid size.

$\varepsilon \quad$ Dissipation rate for the turbulent kinetic energy per unit mass, $\mathrm{K}$ (single-point).

$\checkmark \quad$ Specific volume.

$\xi$ A coordinate in the invariant coordinate system (self-similar variable).

$\eta \quad$ A coordinate in the invariant coordinate system (self-similar variable).

$\rho \quad$ Instantaneous fluid density.

$\bar{\rho} \quad$ Average fluid density.

$\rho^{\prime} \quad$ Fluctuation of density away from average fluid density.

$\rho_{i} \quad$ Microscopic density of the $i-t h$ fluid.

$\sigma$ Diffusion coefficient to regulate the stability of the two-fluid equations.

$\sigma_{\mathrm{ij}}$ Stress tensor.

$\sigma_{\varepsilon}$ Model constant in the single-point S-equation pertaining to the Prandtl number.

$\tau_{\mathrm{ij}} \quad$ Viscous stress tensor.

$v \quad$ Kinematic viscosity.

$v_{\mathfrak{t}}$ Turbulent, or eddy, viscosity.

$\mu \quad$ Molecular viscosity.

$\Xi \quad$ Variable used by Kucherenko et al. (1991) to plot the volume fraction throung the mixing zone, effectively reduces the influence of the long tails of fluid.

$\omega$ Scaling group parameter. 
. 


\section{Background and Motivations}

\subsection{Introduction}

Turbulence, which occurs in many circumstances of fluid flow, is driven or sustained by the conversion of large-scale mean flow energy to intermediate-scale fluctuations and dissipated by the entropy-increasing process of cascade to small scales, ultimately to the molecular level where it is manifested in the form of heat.

Our goal is to investigate the nature of turbulence by means of extended transport approaches by using two-point (spectral) techniques. In particular, we seek "generic" spectral form functions by which to describe the turbulence structure, especially in circumstances of inhomogeneous, anisotropic drive. These functions may be exact or approximate, depending on circumstances to be described below. They are usually revealed in greatest clarity under circumstances that are described by self-similar combinations of the physical and spectral variables, but they may also occur to a significant level of approximation in localized regions that are continually approaching self-similarity despite the shifting nature of the mean-flow drive.

This work is focused on flows with large variations in fluid density (e.g., two-field fluid interpenetration). The starting point for analysis is the set of Navier-Stokes equations, for which we assume relevance in our investigations, even in the presence of sharp density variations between fluids. Models for two-field analysis with drag representations for momentum exchange can also be used and are discussed in Chapter 4.

In this report departures from mean flow are included in the stochastic concept of turbulence. Reynolds decomposition into mean and fluctuating parts is carried out in the spirit of this generalized concept, which is meaningful despite arbitrariness as to which scales are identified as mean flow and which are identified as fluctuations. (We actually derive much useful guidance by shifting our viewpoint in this regard as discussed in Section 5.2.)

Insertion of Reynolds decomposition into the Navier-Stokes equations and ensemble averaging (using mass-weighted averages), leads to an unclosed hierarchy of transport equa- 
tions for correlations at all orders. Written for a single point in space and supplemented by closure derivations or postulates, the results have proved useful for describing numerous circumstances of two-fluid turbulence (Andronov et al. 1982; Besnard, Harlow, and Rauenzahn, 1987). [This single-point model proposed by Besnard et al. (1987) is hereafter referred to as the BHR model.] There are, however, some significant elements missing from this approach.

A clue to these missing elements lies in considering the much better known turbulence transport equations for constant-density fluid flow. It has long been recognized that a singlepoint transport equation for Reynolds stress must be supplemented by a transport equation for an auxiliary quantity, often chosen to be the dissipation tensor, $\varepsilon_{i j}$, or its contraction, $\varepsilon$. Previously identified (Daly and Harlow, 1970) as the Reynolds-stress sink caused by molecular viscosity, $\varepsilon$ is more accurately associated with the cascade flux of energy from low to high wave numbers. Recognizing the necessity for a transport equation of this dissipation tensor provides motivation for considering a two-point generalization of the Reynolds stress, from which we can derive the spectral transport equations by Fourier transformation. This spectral representation does not require a supplemental transport equation for scale because it describes the continuously evolving distribution of Reynolds stress across all wave numbers. In particular it confirms the interpretation of $\varepsilon$ as the flux from the dominant lower-wave-number parts of the spectrum through a more-or-less inertial (or Kolmogorov, 1941) middle range to high wave numbers. Viscous dissipation may occur at even higher wave numbers at a rate that is not necessarily in equilibrium with the cascade flux. A transport equation for $\varepsilon$ can be derived as a moment of the full spectral equations provided that an appropriate spectral form can be discovered for the spectral tensor, as discussed in Appendix E.

With large density differences in the fluid, the spectral analysis becomes much more complicated, especially because we include density variations that arise from the interpenetration of two completely immiscible materials: bubbles in a liquid, solid grains in a gas, or two immiscible fluids interacting in a general configuration of swirling filaments and droplets. The Navier-Stokes equations are assumed to be relevant for all three of these cases. For the case of 
solid grains dispersed in a gas, the regime of validity for this study is limited to the case where the grains of solids are small enough to treat the cloud of dispersed phase particles in the gas as a separate fluid.

At this stage of theoretical development, the attainment of a rigorous formulation even for constant density flows is not possible. During the last three decades of constant-density turbulence-transport developments, the derivations have had to combine empiricism with rigor, and the same is true for the variable-density extensions. Constraints (dimensionality, preservation of conservation, tensor form, Galilean invariance, simplicity of form, and similar general considerations) serve to limit the choices for modeling. Comparisons with experiments likewise furnish guidance.

In this report we describe a spectral turbulence transport formulation for variable-density turbulence in circumstances of inhomogeneity and anisotropy. We show that moments of these equations introduce some auxiliary single-point variables. These are some of the elements that are missing from previous single-point formulations for variable-density turbulence (e.g., the BHR model). We also describe some comparisons with experiments of the spectral formulation and indicate numerous directions for refinement, analogous to those that are required for constant-density turbulence transport.

The family of model-development work into which these variable-density turbulence activities fit is shown in the diagram of Fig. 1.1.1. The starting point for all directions is the set of Navier-Stokes equations.

Guidance for this spectral model is taken from these three branches of transport modeling, namely (1) the simple (local) closures describing transfers in k-space from the two-point branch [Besnard, Harlow, Rauenzahn, and Zemach, 1990 (hereafter called the BHRZ model); Clark and Spitz, 1995], (2) the single-point, single fluid, turbulence transport models, and (3) the single-point, two-field models. Another branch from the two-point box which could be shown handles the transfers of energy in k-space with much detail, but no use is made of 
that approach for this model so it is not shown. Likewise, other branches that exist are not shown, since the ancestry of this model is composed of only these three branches.

Another branch that evolves directly from the Navier-Stokes equations leads to the twofield (or multifield) flow equations. Explicit modeling describes the principal interaction between discrete entities and fluid in terms of drag. Within the two-field formulation, the fluid interpenetration is described by terms in the equations that contain no contributions from

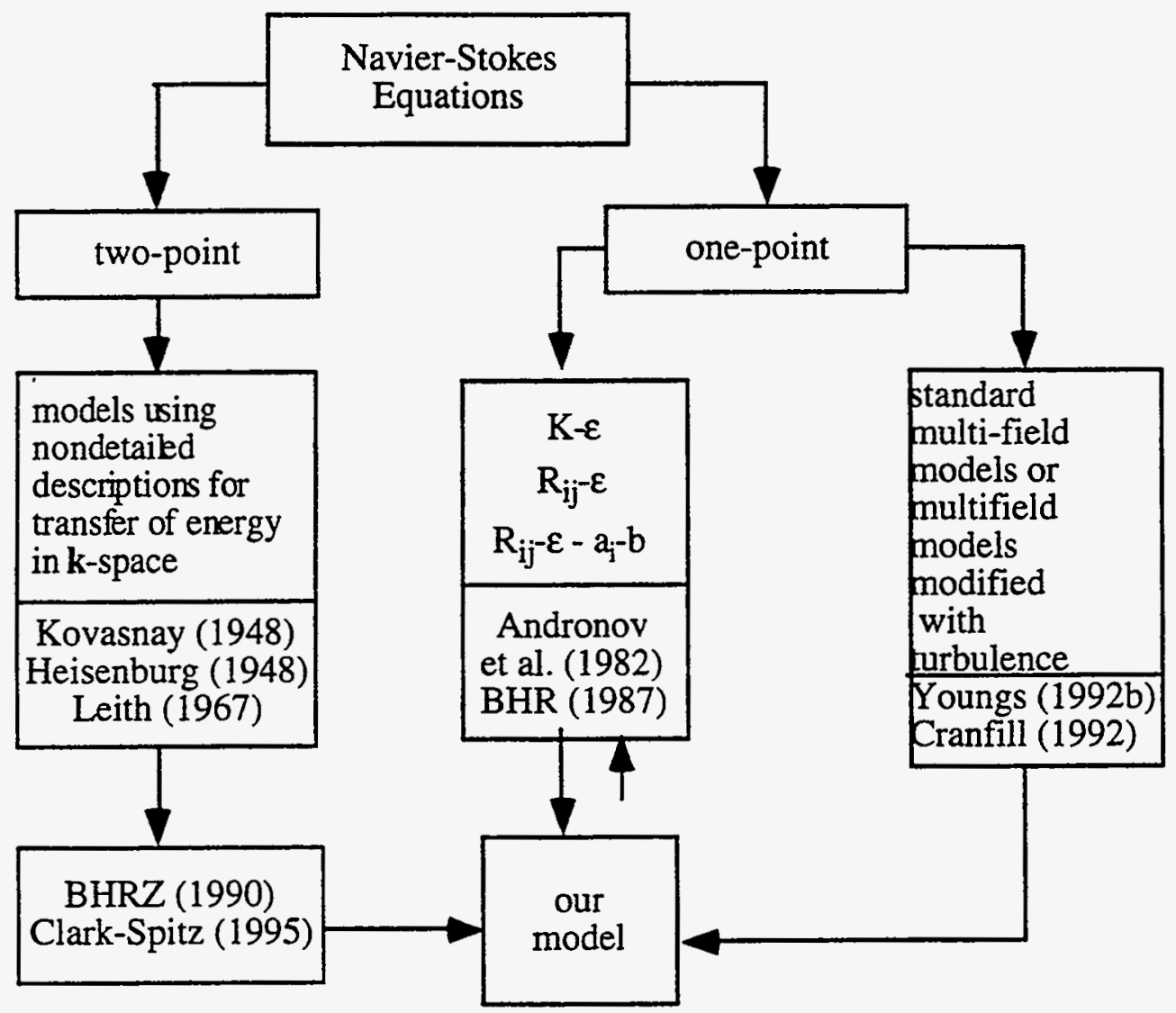

Figure 1.1.1. Flow chart defining the ancestry of the our spectral transport model .

fluctuations away from the mean within each field. This interpenetration is an "ordered" process (see Appendix C). In addition, a "disordered" component of the interpenetration can be expected to arise as a result of fluctuations away from the mean flow. The turbulence transport equations are capable of representing this "disordered" component of interpenetration. The two-field equations are capable of exhibiting the origin of these fluctuations. They are unstable whenever there is relative motion between the two fluids. Thus, the two-field formulations 
contain hints of a disordered component to interpenetrating flow but require further modeling for practical exploitation of this feature. As usually written, two-field models are incapable of representing crucial aspects of the general turbulent dynamics that we seek to describe.

Directly descendent from the two-field models are those that have been extended to include the missing processes. These modifications are derived through empirical and heuristic arguments. They include

1. enhancement (by an order of magnitude with respect to a single sphere) of inter-field momentum exchange (drag) coefficients;

2. incorporation of an evolution equation for the effective length scale, which represents the effects of disperse phase clumping into larger and larger sizes during interpenetration;

3. the addition of a disordered component, and the diffusive effects thereof, with energy source proportional to the loss that results from drag.

Another branch in the diagram of Fig. 1.1.1 shows the development of single-point turbulence transport equations, also directly from the Navier-Stokes equations. These representations of the fine-scale fluctuational behavior of the fluid bear a close analogy to the descriptions of molecular dynamics expressed by the Navier-Stokes equations themselves. Just as the equations for gas dynamics are obtained from moments of a Boltzmann equation, so also the turbulence transport equations are formulated by means of moments of the Navier-Stokes equations. The parallel is illustrated in Fig. 1.1.2.

Much work has been directed over the last three or more decades towards development of the single-point turbulence transport branch for a single fluid with constant density. The two-point (spectral) branch, which represents the principal focus for this report, has its roots in the work of Heisenberg (1948), Kovasnay (1948), and Leith (1967, 1971), and more recently Cambon (1979), Cambon, Jeandel, and Mathieu (1981), Bertoglio (1982), BHR (1987), and Clark and Spitz (1995).

At the bottom of the chart of Fig. 1.1.2 we show one of the ultimate products to which this research can be directed, the generalized single-point transport equations with the 
necessary additional features for realistic and practical problems solving over a wide scope of circumstances. Achievement of this goal requires extensive consideration of issues related to the spectral structure of "turbulence," in its most general sense.

- ordered and disordered components of the behavior;

- anisotropic structures in both physical space and wave-number space;

- self-similarity.

\begin{tabular}{|c|c|c|}
\hline & Molecular Dynamics & Turbulence Transport \\
\hline $\mathrm{A}$ & \begin{tabular}{|l|} 
Many-particle distribution-function trans- \\
port, based on $d x_{i} / d t=u_{i}$ and \\
$m d u_{i} / d t=F_{i}$ \\
\end{tabular} & $\begin{array}{l}\text { The "exact" equations for fluid dynamics, the } \\
\text { Navier-Stokes equations }\end{array}$ \\
\hline $\mathrm{B}$ & $\begin{array}{l}\text { BBGKY reduction to transport equations } \\
\text { for } N \text {-particle distribution functions in- } \\
\text { volving integrals over } N+1 \text {-particle dis- } \\
\text { tribution functions (exact but unclosed; } \\
\text { but is the process convergent?) } \\
\downarrow\end{array}$ & $\begin{array}{l}\text { Reynolds decomposition (two-point) with en- } \\
\text { semble-averaged transport of } \mathrm{N}^{\text {th }} \text {-order correla- } \\
\text { tions involving integrals of } \mathrm{N}+1^{\text {st }} \text { order cor- } \\
\text { relations (exact but unclosed; but is the process } \\
\text { convergent? }\end{array}$ \\
\hline $\mathrm{C}$ & $\begin{array}{|lc|}\text { "Chaotic" assumption; reduction to } \\
\text { Boltzmann equation } & \downarrow \\
\end{array}$ & $\begin{array}{l}\text { QN approximations plus closure modeling } \\
\text { techniques }\end{array}$ \\
\hline $\mathrm{D}$ & $\begin{array}{l}\text { Single-relaxation-time model for integral } \\
\text { terms; often serves as a good approxima- } \\
\text { tion for Boltzmann equation } \\
\downarrow\end{array}$ & $\begin{array}{l}\text { Spectrally local representations for cascade, re- } \\
\text { turn to isotropy, and other processes }\end{array}$ \\
\hline$E$ & $\begin{array}{l}\text { Small-departures from equilibrium to give } \\
\text { distribution-function forms for moment } \\
\text { equations (Chapman-Enskog for the stress } \\
\text { tensor) leading to Navier-Stokes equations } \\
\text { (requires experimental determination of } \\
\text { transport functions) }\end{array}$ & $\begin{array}{l}\text { Locally self-similar form functions for spectral } \\
\text { moment integrals to give single-point transport } \\
\text { equations (e.g., } R_{\mathrm{ij}}-\varepsilon_{\mathrm{ij}} ; \mathrm{K}-\varepsilon \text {; BHR forms) } \\
\text { (requires experimental determination of trans- } \\
\text { port functions) }\end{array}$ \\
\hline $\mathrm{F}$ & $\begin{array}{l}\text { Becomes the starting point for the right } \\
\text { column }\end{array}$ & $\begin{array}{l}\text { Quasi-equilibrium approximations for local } \\
\text { point-functional representations (e.g., mixing- } \\
\text { length forms). (Resembles closure modeling } \\
\text { techniques for gradient-flux expressions and for } \\
\text { approx. of triple-correlation transport in } \\
\text { EDQNM) }\end{array}$ \\
\hline
\end{tabular}

Figure 1.1.2. Diagram showing the "family tree" for turbulence models. 


\subsection{Research summary}

This section serves to summarize the motivations, goals, and contributions of the research herein. Coupled with Section 1.3 and the literature survey of Section 1.4, this section sets a perspective as to which issues of turbulence transport modeling we address, the strategies we use, and the technical contributions of this work.

\subsection{A Motivations}

Turbulent flows contain a wide range of length scales and time scales. The production of turbulent kinetic energy is identified with the relatively large scales of the flow. Turbulent kinetic energy is transferred from these relatively large scale structures, or eddies, to increasingly smaller and smaller structures until finally the molecular viscosity of the fluid dissipates the energy to heat at the smallest scales of the flow. This process by which energy cascades from the large eddies down through the intermediate scales (inertial range) to the fine (viscous dissipation range) scale structures is very complicated. This cascade process is identified as the primary physical mechanism by which turbulence is removed from the size scales of significance.

As opposed to the single-point models, two-point models carry spectral information about each turbulence variable. The information provided in these spectral distributions represents a vast enhancement over the amount of information provided by single-point models. Spectral models have been developed for constant density turbulence, and even variable density homogeneous turbulence, but never for the case of variable density inhomogeneous turbulence.

Spectral self-similarity is a powerful concept. This allows for a turbulent flow field that is composed of eddies of many different length scales and different strengths to be represented by one length scale, one kinetic energy, and a tensor structure that is uniquely determined by the geometrical structure of the driver. Von Karman and Howarth (1938) were the first to suggest a self-similar form function for the turbulent kinetic energy spectrum of isotropic turbulence, $E_{n n}(k, t)=K_{n n}(t) L(t) f(\xi)$. In this expression $E_{n n}(k, t)$ is the turbulent kinetic energy 
spectrum, $K_{n n}(t)$ is the turbulent kinetic energy, $L(t)$ is the length scale, which is equal to the reciprocal of $\mathrm{k}_{\max }$ (the wave number at which $\mathrm{E}_{\mathrm{nn}}(\mathrm{k}, \mathrm{t})$ attains a maximum), $\mathrm{k}$ is the Fourier wave number, $\mathrm{f}$ is a spectral form function, and $\xi=\mathrm{kL}(\mathrm{t})$ is the self-similarity variable. In $\xi$ space, $f(\xi)$ is independent of time.

Given an entire spectrum of wave numbers, a distribution of the Reynolds stress tensor can be identified over this spectrum. We call this the spectral form of the Reynolds stress tensor. For a turbulent flow that has reached equilibrium, the spectral form of $R_{i j}$ remains unchanged. That is to say, a balance is established among the different production and cascade terms. In this case the turbulence might also be in a state of spectral self-similarity. After appropriate scaling, the evolution of the entire $R_{i j}(k, t)$ spectrum can often be adequately represented by one spectral form function (referred to here as a generic form function). The shape of the generic form function is constant in time.

In order to approach spectral self-similarity (Besnard et al. 1990), three conditions must be satisfied: (1) the flow must contain turbulent Reynolds numbers large enough to accommodate a well defined inertial range in the wave number spectrum, (2) the characteristic time scales for distortions in the spectral form functions due to changes in the mean flow strain rates must be much greater than the eddy turnover times for the dominant scales in the turbulence, and (3) the mean flow must respond to the turbulence in a manner that is consistent with the self-similarity.

The identification of self-similar form functions for the turbulence allows for the derivation of simpler one-point models from the two-point models, as discussed in Appendix E. The result is a single-point model that has been enhanced with physical insight not available through the conventional manner of derivation of single-point models. Singlepoint models are more appealing to the engineering community for reasons of computational efficiency, whereas two-point models require the resolution of the additional dimension in kspace. 
Although spectral modeling has been developed for constant density turbulence as well as variable density homogeneous turbulence, a two-point model for variable density inhomogeneous turbulence has not previously been developed. In this study we have developed such a spectral model. We consider this new formulation and the study of its consequences to be major contributions. The flavor of our model lies between the rigor of formal mathematical derivations on one side and the more simple engineering type single-point models on the other. We also derive from this spectral formulation a single-point model for inhomogeneous variable density turbulence. This is done through the identification of self-similar form functions and spectral integration of the two-point model equations. A single-point model developed in this fashion is strengthened by the physical enhancements because of its spectral ancestry.

\subsection{B Goals}

- Use every relevant facet of previous developments in transport modeling as a basis for constructing a tractable two-point spectral model for inhomogeneous variable density turbulent mixing.

- Enhance the physics of the model by incorporating a nonlocal pressure source term into the $\mathrm{R}_{\mathrm{ij}}$ transport equation.

- Demonstrate validity of the spectral models by comparison with experiment.

- Identify spectral self-similarity in the mixing layer problem.

- Identify departures from self-similarity due to rapid transients in the flow.

- Show applicability of the model to circumstances with rapid transients in the external drive when the spectra are far from equilibrium.

- Identify a whole new class of generic self-similar form functions for inhomogeneous anisotropic turbulence.

- Develop a new single-point model through spectral integration of the new spectral model. 


\subsection{Strategy}

- Come to grips with some troubling mixing issues in the single-point arena.

- Examine single-point modeling strategies for guidance in two-point closures.

- Review the effects of two-fluid modeling on single-point turbulence transport closures.

- Use a hybrid model which combines a single-point turbulence transport model with a two-fluid transport model to resolve some mixing problems of the single-point turbulence transport model.

- Examine and extract any possible guidance from single-point modeling strategies for two-point closures.

- Examine the exact unclosed two-point turbulence transport equations for variable density inhomogeneous flows.

- Realize that a rigorous theoretical derivation is impossible at this time.

- Identify all of the constraints imposed by nature that one must adhere to in constructing a credible model of such a physical processes.

- Adopt the convention of simplicity when examining candidate model terms.

- The resulting model should represent the "bare bones" example of what will capture a majority of the physics.

- Collect and apply all relevant well-tested modeling strategies from previous investigators .

- Develop a formalism to handle the nonlocal effects of pressure for an incompressible flow.

- Validate the model by comparison to experiment.

- Identify self-similarity behavior from the model.

- Physical space.

- Spectral space.

- Use spectral moments to derive an improved single-point engineering model. 


\subsection{Contributions}

- For the first time, a working tractable spectral model for variable density inhomogeneous turbulent mixing.

- Provides a basis upon which to extend spectral investigations of nonlocal process in both wave number and physical space. Provides a direction for future theoretical work to bridge the gap between rigorous derivations and this model.

- New information on spectral self-similarity in the presence of inhomogenieties and strong anisotropies.

-We supply evidence that a principle exists to explain the phenomena we are observing; self-similar form functions in anisotropic inhomogeneous flows in which the spectral forms of the different components of the anisotropic $R_{i j}$ are all roughly the same, independent of the position in the mixing layer.

- A new single-point model for variable density inhomogeneous turbulence that has inherited an enrichment of physical information from the spectral model.

- Previous single-point models have no previous basis for describing flows out of spectral equilibrium. The single-point model derived in this dissertation is an advancement over previous models due to the fact that our new single-point model transports a length scale for each variable that has been derived from spectral information.

- Formulation of an enhancement to the pressure gradient source term to the $R_{i j}$ transport equation. This model enables the pressure to influence the flow in a global fashion, a fact which accounts for the consequences of infinite signal propagation rate in an incompressible flow, and thereby, corrects a large error in previous spectral formulations and allows for proper representation of linear instability growth at small wave numbers (e.g., classical Rayleigh-Taylor instability). 
- Produced spectral data that can be used by experimentalists to guide data collection and interpretation, for example, in the new equipment used by researchers at Lawrence Livermore National Laboratory for material mix studies.

\subsection{Chapter summaries}

Chapter 1 introduces the topic of this dissertation. Section 1.2 discusses the motivations behind the formulation of a spectral turbulence transport model for mixing. We discuss the assumption of spectral self-similarity which is implicit in the formulation of a single-point transport model and describe the circumstances in which a single-point model breaks down. In Section 1.2 we point out the advantages of a spectral model. The survey of Chapter 1 reviews the literature for the Rayleigh-Taylor instability and turbulence transport modeling. The literature survey serves to put the spectral model of this dissertation in the proper context with respect to the other turbulence transport models. The survey also introduces the experimental data that we compare to in this dissertation.

In Chapter 2 we define the turbulence variables that are transported in the models discussed in this dissertation. The single-point BHR turbulence transport model (described in Chapter 3) and the two-point spectral model formulated in Chapter 5. Both transport the same quantities. The variables are derived in their two-field equivalent forms. We call these the configurational form of the variables. We use the simple one-dimensional mixing layer to introduce and attach physical meaning to these variables. Hence, the configurational equivalent of each variable is derived for the case of two interpenetrating immiscible fluids.

Chapter 3 is used to demonstrate the strategies behind single-point closures for variable density turbulence. This chapter is include because some of these strategies were consequently incorporated into the development of the spectral model of Chapter 5. The single-point BHR model (1987) is used for this demonstration. As background investigation for this dissertation, much time was spent trying to improve single-point modeling by trying two-fluid model closures for the transport model. Numerical solutions for the Rayleigh-Taylor mixing problem are 
discussed. In Chapter 3 we also point out the assumptions that go into single-point modeling which present as motivation for a spectral model.

As part of the background investigations, we attempted to improve the single-point turbulence transport model of Chapter 3. Diffusive closures are used to close the higher-order correlations in the BHR model. The closures of two-field flow equations are discussed in Chapter 4. We show that with certain modifications, the two-field flow equations can be made to give decent results and can properly describe demixing. We summarize by explaining that even though two-field results can be made to give good results for the mixing layer, the appeal of a density fluctuation as opposed to volume fractions used to identify parcels of fluid motivate use to stick with the turbulence transport equations for the basis of a spectral model.

Chapter 5 is dedicated to the discussion of the formulation and origin of each term in our spectral model. We discuss the strategy we use in the formulation as well as the constraints that must be followed in order to keep the model credible.

Chapter 6 gives a discussion on the terms in the model, for both physical space as well as spectral space.

Numerical results are given in Chapter 7 for the case of a Rayleigh-Taylor mixing layer. We also compare favorably to the experiments of Smeeton et al. at the British Atomic Weapons Establishment (AWE) and of Kucherenko et al at Chelyabinsk-70.

Chapter 8 summarizes the work presented in this dissertation.

The appendices are used to enhance the text with issues that would make reading the main text of this dissertation cumbersome if they were to be included. Appendix A demonstrates that the single-point BHR model conserves energy. Since the spectral model is developed to be conservative in k-space and the same strategies are used for closure in both models than the conservative nature of the BHR model can be extended to the model developed in Chapter 5. A discussion is given in Appendix B on the derivation of the of an enhanced source term to the single-point b-equation of the BHR model. Appendix C attempts to explain the concept of an ordered and a disordered component of an interpenetrating flow. 
Appendix D demonstrates that by dimensional arguments we can formulate the self-similar form functions for our Reynolds stress tensor. This formulation is then used in Appendix $E$ to take the spectral moments of the spectral equations to derive a single-point set of equations. The numerical schemes are given in Appendix F for the numerical solution of : (1) the BHR turbulence transport model discussed in Chapter 3, (2) the two-field flow model discussed in Chapter 4, and (3) the spectral model developed in Chapter 5.

\subsection{Literature survey}

In Chapter 5 of this report, we present a set of spectral turbulence transport equations used to describe the turbulent mixing of two incompressible fluids. In Chapter 6 we discuss the properties of these equations. As a benchmark problem, in Chapter 7 we analyze the nonlinear, fully developed self-similar stage of turbulent mixing of two fluids initiated by the Rayleigh-Taylor instability. We also use this same problem in the single-point discussions of Chapters 3, and 4. Data also exists for the incompressible turbulent mixing of two fluids due to a free shear layer (Brown and Roshko, 1974), but the scope of this work is limited to the turbulent mixing due to the Rayleigh-Taylor instability.

This literature survey is presented in two parts. The first part is used to review the previous investigations of the Rayleigh-Taylor instability and the consequential two-fluid turbulent mixing. Within the first part, we review the literature of relevant experimental data of Rayleigh-Taylor mixing that is available for comparison. We also use this survey to define important concepts for the rest of this report. In the second part, we survey the evolution of turbulence modeling to set a perspective for the contributions of this work with respect to other turbulence models.

\subsection{A Two-fluid turbulent mixing due to the Rayleigh-Taylor instability}

The Rayleigh-Taylor instability occurs at the interface of two fluids of different densities configured such that the lighter fluid is accelerated by a pressure gradient into the heavier fluid, i. e., whenever the pressure gradient opposes the density gradient $(\nabla \bar{p} \cdot \nabla \bar{\rho}<0)$. This 
can occur, for example, in ICF targets during the laser implosion of a dense shell (pusher) filled with deuterium-tritium gas. The effects of this instability during the acceleration phase are of current interest to the ICF community (Takabe, et al. 1985). For this study, we consider the incompressible case of the Rayleigh-Taylor instability where a body force due to an acceleration produces a pressure gradient across the fluid-fluid interface such that the higher pressure occurs in the lighter fluid. The reason that this point is emphasized here is that a similar instability can also occur at an interface due to the passage of a shock wave (Richtmyer, 1954 \& 1960; Meshkov, 1969; Andronov et al. 1976). Richtmyer investigated the small amplitude theory for this case, and Meshkov performed the related shock tube instability experiments. The distinction is made because we investigate the case of a continuous (pressure gradient) driver with incompressible fluids (Rayleigh-Taylor instability) rather than an instantaneous (shock wave) driver with compressible fluids (Richtmyer-Meshkov instability).

Given an interface between two different fluids with gravity oriented so as to create a higher pressure in the lighter fluid, the lighter fluid could, in theory, support the heavier fluid with no mixing if the boundary interface is perfectly smooth with no perturbations. In nature, however, a perfectly smooth interface is practically impossible. Neglecting surface tension or viscosity, all interfacial perturbations begin to grow exponentially, and it is the smallest perturbations (those corresponding to the largest wave numbers) that dominate the growth of the Rayleigh-Taylor instability at the interface.

Using the linearized form of the dynamical equations (which is only possible for small perturbations), Chandrasekhar (1961) shows that in the absence of viscosity the amplitude, a, of a small sinusoidal perturbation of wavelength $\lambda$ on the interface between two fluids grows according to the formula

$$
a=a_{0}^{+} \exp \left(n_{\lambda} t\right)+a_{0}^{-} \exp \left(-n_{\lambda} t\right),
$$

where the growth rate $n_{\lambda}$ is given by

$$
\mathrm{n}_{\lambda}^{2}=\frac{2 \pi \mathrm{g}}{\lambda} \frac{\rho_{1}-\rho_{2}}{\rho_{1}+\rho_{2}}
$$


For a configuration of fluid\#2 sitting over fluid\#1, the configuration is unstable, i.e., it grows exponentially, for two cases: (1) $\rho_{2}>\rho_{1}$ with $g<0$; or, (2) $\rho_{1}>\rho_{2}$ with $g>0$. The configuration is stable, i.e., the solution oscillates, for two cases: (1) $\rho_{1}>\rho_{2}$ with $g<0$; or, (2) $\rho_{2}>\rho_{1}$ with $g>0$. Chandrasekhar (1961) also shows that, for the case with viscosity, $\mathrm{n}_{\lambda}$ reaches a maximum value, $\mathrm{n}_{\mathrm{m}}$,

$$
\mathrm{n}_{\mathrm{m}}=\sqrt{\frac{\pi \mathrm{g}}{\lambda_{\mathrm{m}}}\left(\frac{\rho_{1}-\rho_{2}}{\rho_{1}+\rho_{2}}\right)}
$$

where $\lambda_{\mathrm{m}}$,which corresponds to a most unstable wavelength, is given by

$$
\lambda_{\mathrm{m}} \approx 4 \pi\left\{\frac{v^{2}}{\mathrm{~g}} \frac{\rho_{1}+\rho_{2}}{\rho_{1}-\rho_{2}}\right\}^{1 / 3} .
$$

Here, the mean kinematic viscosity, $v$, is given by

$$
v=\frac{\mu_{1}+\mu_{2}}{\rho_{1}+\rho_{2}} .
$$

From the above solutions, it can be seen that for the case where random perturbations of many wavelengths are present on the interface, it will be the shorter wavelengths that will dominate the growth. Thus for the inviscid case, the short wavelength, small perturbations dominate the long wavelength small perturbations in the growth rate of the instability. This growth rate goes to infinity as $\lambda \rightarrow 0$. For the viscous case, the minimum size of the perturbation wavelength, $\lambda_{m}$, is mitigated by the effect of viscosity, which prohibits the growth rate of the instability from going to infinity.

Youngs (1984) describes the "basic picture of the mixing process" due to the RayleighTaylor instability in which he identifies three stages of evolution for the instability. The first stage that he identifies corresponds to the appearance of the most unstable perturbation of wavelength $\lambda_{\mathrm{m}}$, occurring about two or three times the characteristic time, $\tau_{\mathrm{m}}$, after the body force has been applied to the system, where $\tau_{\mathrm{m}}=1 / \mathrm{n}_{\mathrm{m}}$. Stage two of the evolution is characterized by a slowing of the growth rate of the amplitudes of the perturbations that penetrate the denser fluid from exponential to a rate proportional to $\sqrt{\mathrm{g} \lambda}$. Stage two is characterized by the 
classical Rayleigh-Taylor picture of interpenetrating spikes and bubbles and commences when the height of the perturbation is approximately half the value of the wavelength. If the initial perturbations are small, then it is the nonlinear coupling between the small scale structures that creates larger wavelengths. These interactions facilitate the loss of memory of the initial conditions. All memory of the initial conditions is lost when the dominant wavelengths of the perturbations are about 10 times larger than $\lambda_{m}$. During this period of the mixing, spikes of the heavier fluid penetrate the lighter fluid while round ended columns of bubbles of the lighter fluid penetrate the heavier fluid. This marks the beginning of the final stage of evolution. The effects of viscosity diminish as the scale structures continue to grow.

Once the perturbation amplitudes have reached stage two, the experiments of Lewis (1950) and Emmons et al. (1960) show that it is a competition between bubbles that leads to a bubble amalgamation process where the larger bubbles crowd out the smaller ones. The velocity derived in the theory by Davies and Taylor (1949), $\mathrm{v}=(2 / 3) \sqrt{\mathrm{gR}}$ also shows that the bubbles of larger radius will dominate the flow. This process by which dominant wavelengths increase to infinity is also referred to as bubble doubling.

In the final stage, the flow (for the mixing region) is defined as completely turbulent, and it is this mixing zone between the two fluids that is referred to hereafter as the Turbulent Mixing Zone (TMZ). Since all memory of the initial conditions is lost at the beginning of this stage, the flow is said to be self-similar. Consequently, the only remaining identifiable length scale of the flow is the quantity gt ${ }^{2}$. Thus, the width $\mathrm{W}$, of the TMZ can only be expressed as a product of this quantity and some function of the density, namely,

$$
W=F\left(\frac{\rho_{1}}{\rho_{2}}\right) g t^{2} .
$$

Youngs (1984) cites a simple argument to qualitatively support this dependence on the density ratio. He supposes that throughout the final stage of growth, the dominant wavelength take $\mathrm{N}$ exponential growth periods to appear. Then from Eq. (1.4.2) and Eq. (1.4.1) it follows that 


$$
\mathrm{W} \propto \lambda_{d}=\frac{2 \pi}{N^{2}} \frac{\rho_{1}-\rho_{2}}{\rho_{1}+\rho_{2}}\left(\mathrm{gt}^{2}\right) .
$$

The previous two paragraphs (due to Youngs (1984)) serve us well in illustrating the stage of the Rayleigh-Taylor instability that is of interest to this study. It is the final self-similar (stage 3) stage of turbulent mixing on which this work is focused. It also leads nicely into the identification of three very important variables that are used throughout this report, and they are $\mathrm{h}_{1}, \mathrm{~h}_{2}$, and $\alpha$.

By definition, the width, $W$, of the TMZ is the algebraic sum of $h_{1}$ and $h_{2}$ such that, $W=h_{1}+h_{2}$. The definition of $h_{1}$ is the difference in height between the position of the original undisturbed interface and the position where the volume fraction of the heavy fluid has decreased from 1.0 to something near 0.95 , i.e., near the edge of the bubble envelop; whereas $\mathrm{h}_{2}$ is defined as the height difference between the position of the original undisturbed interface and the position where the volume fraction of the light fluid has decreased from 1.0 to something near 0.05 (Smeeton \& Youngs, 1987; Read \& Youngs, 1983; Youngs, 1992a). The exact volume fraction that is used to determine $h_{1}$ and $h_{2}$ differs among experimenters. Kucherenko et al. (1991) define $L_{21}$ for the British value of $h_{2}$ and $L_{12}$ for the British value of $h_{1}$, but for simplicity, the data of Kucherenko, et al. is re expressed in terms of $h_{1}$ and $h_{2}$ within this report.

Both numerical simulations (Youngs, $1984 \& 1991$ ) and experimental results (Smeeton \& Youngs, 1987; Youngs, 1992a; Read, 1984; Read \& Youngs, 1983) indicate that Eq. (1.4.6) is more applicable to the depth of penetration of the lighter fluid (bubble envelop) into the heavier fluid, $\mathrm{h}_{1}$, than for the total width of the TMZ, W. Read and Youngs (1983) and Burrows et al.. (1984) show that if the mixing between the two fluids evolves from small random perturbations, the penetration of the mixing region into the denser fluid, $h_{1}$, is given by

$$
\mathrm{h}_{1}=\alpha \frac{\rho_{1}-\rho_{2}}{\rho_{1}+\rho_{2}} \mathrm{gt}^{2},
$$

where the self-similarity coefficient $\alpha$ was found to be insensitive to the density ratio and varied little from a value of about 0.06 among different experiments. One final definition that is 
used throughout this report is that of the variable $X$, the scaled acceleration used in the above experiments, which is given by

$$
\mathrm{X}=\frac{\rho_{1}-\rho_{2}}{\rho_{1}+\rho_{2}} \mathrm{gt}^{2}
$$

or, $X=(A t) g t^{2}$, where $(A t)$ is the Atwood number and $\rho_{1}>\rho_{2}$.

Many authors have investigated the early stages of the Rayleigh-Taylor instability. A quantitative overview of the Rayleigh-Taylor instability which describes the phenomenology that occurs at an unstable interface is given by Sharp (1984). Lord Rayleigh (1883) was one of the first to investigate the equilibrium of a stratified inviscid fluid. Lamb (1931) discusses some of the problems related to Rayleigh's paper. Taylor (1950) generalized the linear analysis which took into account inertial and body forces to include the effects of surface tension. Lewis (1950) experimentally confirmed Taylor's findings by accelerating an initially stably stratified fluid downward at a rate 50 times greater than the acceleration due to gravity. This study by Lewis showed that, for an air-liquid interface, the linear analysis was correct for the initial stages of the instability. The experiment also shed some light on the large amplitude evolution of the instability.

This instability has been investigated using infinitesimal perturbations on the initial interface (Lamb, 1931; Taylor, 1950; Allred \& Blount, 1953; Birkhoff, 1954 \&1956), as well as finite amplitude initial perturbations (Birkhoff, 1954 \&1956; Emmons, et al. 1960). The investigations have been extended to include the effects of surface tension (Bellman \& Penningon, 1954), viscosity (Chandrasekhar, 1954; Hide, 1955), gradual density gradients (LeLevier, et al. 1955; Case, 1960), as well as diffusion (Duff, et al. 1962). Mitchner, et al. (1964) extend the above work to include the effects of compressibility.

Experiments that have concentrated on the growth of a single wavelength perturbation due to the Rayleigh-Taylor instability include the work of Emmons, et al. (1960), Cole and Tankin (1973), Ratafia (1973), and Popil and Curzon (1979). Ratafia (1973) experimentally 
demonstrated the existence of Kelvin-Helmholtz roll up structures on the sides of the interpenetrating spikes of the heavier fluid.

The first successful numerical calculations of the early stages of the Rayleigh-Taylor instability were performed Harlow and Welch (1965) using their MAC method (Marker and Cell) for free surface calculations. Welch, et al. (1966) describes a multi-fluid extension to the MAC method which was then used by Daly (1967) to study the influence of density variations and viscosity on the rate of growth and shape of the Rayleigh-Taylor instability. Daly found agreement with the predictions of Chandrasekhar (1954) for the growth rates in the linear regime. Daly investigated the evolution of a single wavelength initial velocity disturbance for various density ratios and established the now well-known behavior of a single wave at large amplitudes. For small density differences, Daly showed how the interface rolls up into two counter rotating vortices. For large density differences, the classical picture of the spikes of the heavier fluid are seen penetrating the lighter, which in turn, bubbles up around the interpenetrating spikes. Daly (1969) extended his work of 1967 to include the influence of surface tension on both the linear and the non-linear phase of the instability.

All of the above investigations have focused on the early stages of the Rayleigh-Taylor instability. This stage, which is amenable to analytic solution, is ideal for model verification and numerical validations. However, the emphasis of this report is on the late-stage, self-similar, turbulent mixing of two fluids which has been initiated by the early stages of the RayleighTaylor instability. Experimental data for the last stage of the instability exists for the evolution of the width of the TMZ, the profile of the density across the TMZ, and the rate at which the bubbles of the lighter fluid penetrate the heavier fluid.

Read and Youngs (1983) and Read (1984) use a rocket-rig apparatus at the British Atomic Weapons Establishment (AWE) in Aldermaston to investigate the behavior of mixing induced by the Rayleigh-Taylor instability. Also, Kucherenko, et al. (1991) use a similar apparatus at the Soviet All-Union Research Institute of Technical Physics at Chelyabinsk-70. Rocket motors are used to accelerate a glass walled tank of a stably stratified solution vertically 
downward constrained by a rail of about 1.5 meters in length. The track is backlit, and pictures of the TMZ are analyzed with a densitometer to determine the evolution of the fluid profiles across the TMZ. Nominal experiments last about $80 \mathrm{~ms}$, and, typically, a series of about six pictures are taken for each experiment. Accelerations as high as $45 \mathrm{~g}_{0}$ are achieved at AWE, and Chelyabinsk-70 claim accelerations as high as $2 \times 10^{4} \mathrm{~g}_{0}$, where $\mathrm{g}_{0}=9.8 \mathrm{~m} / \mathrm{s}^{2}$. Two types of tanks are used in the AWE experiments. One tank is designed for ambient pressures, and one is pressurized to achieve larger density ratios (pressures as high as 10 bars are reported). AWE has the capability of tilting the rig and studying the two-dimensional motion of the interface set up by the acceleration (none of these experiments are considered in this report). Six different combinations of working fluids are used in the AWE experiments, but the only data that is used to compare our TMZ profiles with in this report is the combination of aqueous solution of calcium chloride/hexane (Atwood $=0.267$ ). The AWE apparatus is capable of acceleration, acceleration/coast, and acceleration/deceleration profiles.

Data from both sets of the above experiments (AWE and Chelyabinsk-70) are used as a basis for comparison in this report. These experiments and numerical studies (Youngs, 1984) indicate that the instability becomes self-similar during this latest stage of growth. That is to say, the width of a TMZ that grows from an initial interface of small random perturbations spreads in proportion to $\mathrm{gt}^{2}$, where $\mathrm{t}$ is time and $\mathrm{g}$ is the acceleration of the apparatus. The depth of penetration of the bubbles of lighter fluid, $\mathrm{h}_{1}$, into the heavier fluid is described empirically (Smeeton \& Youngs, 1987) as $h_{1}=\alpha X$, where $X=$ Atwood*g*t ${ }^{2}$. The Atwood number is a dimensionless number between zero and one used to describe the density ratio: Atwood $=\left(\rho_{2}-\rho_{1}\right) /\left(\rho_{2}+\rho_{1}\right) . X$ is the self-similarity variable. This relation for $h_{1}$ is valid only after the TMZ has reached self-similarity, i.e., all memory of the initial conditions is lost. In the literature, the depth of penetration of the spikes of heavy fluid into the lighter fluid is defined as $h_{2}$. The self-similar coefficient (acceleration constant), $\alpha$, which defines the slope of $h_{1}$ for the self-similar stage of growth, is found to be insensitive to the density ratio and varies 
little from experiment to experiment (Linden, et al. 1992; Read, 1984; Read \& Youngs, 1983; Andrews, 1992).

The best experimental estimates for $\alpha$ in a truly three-dimensional situation are approximately 0.07 . Youngs (1992a) gives a range of values from 0.050 to 0.077 for $\alpha$. Linden, et al. (1992) report a value of 0.070 for $\alpha$ when using a relatively large, three-dimensional, tank. Andrews and Spalding (1990) cite a value of 0.04 for $\alpha$. Snider and Andrews (1994), who report values of $\alpha=0.070 \pm 0.011$, explain that the container used to hold the mixing fluids of the experiment of Andrews and Spalding (1990) was narrow (approximately "two-dimensional"), and that this could account for the smaller value of $\alpha$. On the average, the values of $\alpha$ are higher for three-dimensional tests than those values of $\alpha$ found in "two-dimensional" tests. Youngs confirms this numerically; for two-dimensional numerical calculations, Youngs (1991,1992a) reports values of a between 0.04 and 0.05 , while the early time values $\alpha$ are reported as higher for the three-dimensional case (Youngs, 1991). Others have numerically investigated the evolution of the TMZ (Andrews, 1992; Gardner, et al. 1988; Glimm, et al. 1992; $\mathrm{Li}, 1993$; Tryggvason \& Unverdi, 1990), and have all reported values of $\alpha$ close to the above values reported by Youngs. The lower extreme of the range being $\alpha=0.038$ and the upper extreme being $\alpha=0.071$.

It should be mentioned that, in the literature, Kucherenko, et al. use a self-similarity variable, $\mathrm{S}$, defined as $\mathrm{S}=\mathrm{gt}^{2} / 2$, but the data from Chelyabinsk-70. that we use in this report is for a density ratio of 3 , which coincides with an Atwood number of 0.5 . Thus, the selfsimilarity variable $\mathrm{X}$ from the British and the $\mathrm{S}$ from the Soviets are identical for the results that are presented in this report.

Demixing has also been investigated (Smeeton \& Youngs, 1987; Kucherenko et al. 1994; Stafford, 1982). Kucherenko, et al., as well as Smeeton and Youngs, accomplish this by changing the acceleration during the stage of self-similar growth of the TMZ. They observe a partial contraction in the width of the TMZ and define a constant to accompany this process which is similar to the acceleration constant of $\alpha$ as described above. They cite values for this 
constant on the order of $1 / 7$ of the values of $\alpha$ given above. Other variants of the change in acceleration are reported by Smeeton and Youngs (1987). Stafford (1982) achieved unstable stratification by heating an initially stably stratified solution of water over saline solution. Buoyancy forces mixed the two fluids, and eventually a partial de-mixing was observed due to the fact that the two fluids did not completely mix at the molecular level. Spalding (1985b) used a direct numerical simulation to study this de-mixing process. Andrews (1986), as suggested by Spalding (1985a) used a two-fluid model to predict both the Rayleigh-Taylor mixing and the late time de-mixing.

Andrews (1986) states that the use of a two-fluid model is necessary because the TMZ is comprised of fragments of different density fluids. A body force induces different accelerations on each fluid fragment causing different relative fluid velocities, and, hence, different inter fluid drags. For a comprehensive summary on two-phase flow modeling, the reader is referred to Drew (1983). Andrews paraphrases Spalding (1985a) by referring to this phenomena as "sifting", and suggests the use of a two-fluid model to capture correctly the relative motion of the fluid fragments. A two-fluid model was necessary as opposed to a turbulence model which uses a diffusive type gradient approximation for closure of the triple terms to capture the effects of a counter-gradient flux. Youngs also uses a two-fluid model to investigate the turbulent mixing of the late stages of the Rayleigh-Taylor instability (1992b). He finds good agreement with his own experiments. For these same reasons, we also incorporate a two-fluid model into our investigations as described later in this report.

Many investigators have used single-point variable density turbulence transport models to investigate the late stages of the Rayleigh-Taylor instability (Anchina, et al. 1978; Andrews, 1986; Anisimov \& Polyonov, 1989; Kucherenko, et al. 1986; Nikiforov, 1991; Polyonov, 1989b; Belenkii, 1965; Neuvazhaev, 1989; Neuvazhaev \& Yakovlev, 1984). Decent agreement with experiment is easily achievable with the adjustment of parameters, but their results are not universal due to the implicit assumptions behind single-point models. Another problem with single-point models is the diffusive nature of the closure assumptions. These diffusive 
closures do not allow for the proper representation of de-mixing (Andrews, 1986) due to any types of transients in the flow. To address this problem, investigators have tried to form hybrid models using both the single-point transport models combined with the equations of twofluid models (Cranfill, 1991; Youngs, 1989 and 1992b).

An avenue that has proved useful in simulating the early stages of the Rayleigh-Taylor instability is numerical simulation using front tracking (or interface tracking) methods (Youngs, 1994; Tryggvason, 1988; Linden, et al. 1994; Li, 1993; Glimm, et al. 1987). Also, the presence of widely varying length scales makes high Reynolds number turbulence very expensive, if not impossible, to simulate using Direct Numerical Simulations. The investigator must have a sufficiently resolved grid in order to compute the transfer of energy to the finest scales of the flows correctly. Due to the complexity of the structures after the self-similar stage has been reached, these methods tend to become very expensive numerically, and one finds motivation to approach the problem from a statistical point of view.

To this date, however, only the investigations of Steinkamp, et al. (1995) have developed a statistical spectral model to examine the self-similar phases of mixing. This spectral model is capable of describing a turbulent flow that is far from self-similarity. This capability goes beyond that which any single-point models may claim.

\subsection{B Turbulence transport modeling}

There is a long legacy of turbulence modeling upon which the model developed in this study is based. This section of the literature survey is intended to give an overview of the evolution of turbulence modeling and the subsequent increase in sophistication as to how one is able to handle the turbulence problem. This section serves to place the model developed in this work in context with other turbulence transport models in the literature, and it also shows a need for a spectral model instead of a typical single-point engineering model. The model developed in this study is by no means the most mathematically sophisticated when compared to other spectral models. However, when developing this model, an attempt is made to capture 
the essence of the physics present in a variable density inhomogeneous mixing situation. In order to capture the transients in general flows such as these one is forced to go to a spectral model, and, in this sense, the model developed herein is more sophisticated than the current single-point turbulence transport models.

For a comprehensive overview of the evolution of turbulent modeling the reader is referred to Markatos (1986) and Launder and Spalding (1972); for further comments on secondorder closures (single-point), see Launder (1990).

The simplest way to close the Reynolds stress tensor in the mean flow equation (after the Navier-Stokes equations have been decomposed into mean and fluctuating parts and ensemble averaged) is to use a simple algebraic expression that relates the mean field behavior to the turbulence. The most well known of these types of models are those that use the Prandtl's mixing length hypothesis.(Anderson, et al. 1984; Bradshaw, et al. 1981). Obviously, since these types of models do not transport any turbulence variables, they quickly break down in situations with flow transients. These models are only useful for flows in which the total effect of the turbulence on the mean flow is small.

For constant density turbulence there is a hierarchy of single-point modeling strategies ranging from the very simple algebraic closure as described above to the two-equation transport models such as the "two-equation-eddy-viscosity" models or the "second order" models. The two turbulence quantities that are typically chosen to be transported in the two-equation models are the turbulent kinetic energy, $\mathrm{K}$, and the dissipation rate of $\mathrm{K}$, namely $\varepsilon$. These models are known as the $K-\varepsilon$ models. Second order models transport the entire Reynolds stress tensor, $\mathrm{R}_{\mathrm{ij}}$, and the kinetic energy dissipation rate, $\varepsilon$.

A single-point two-equation $\mathrm{K}-\varepsilon$ transport model was first proposed by Harlow and Nakayama $(1967,1968)$. Some of the variants of this model that have been proposed include the work by Jones and Launder (1972, 1973), Chien (1982), and Nagano and Hishida (1987). Other two-equation models exist such as models that transport $\mathrm{K}$ times a length scale instead of 
$\varepsilon$ (Ng \& Spalding, 1972), and models that transport the second moment of the vorticity fluctuation instead of $\varepsilon$ (Saffman, 1970; Saffman \& Wilcox, 1974; Ilgebusi \& Spalding, 1985).

Second order two-equation transport models (Rotta, 1951; Daly \& Harlow, 1970; Hanjalic \& Launder, 1972) represent the next step in complexity above the two-equation models just discussed. These types of models transport the full Rij tensor as opposed to only the trace of $\mathrm{Rij}$, where $\mathrm{K}$ is equal to one half the trace of Rij. An advantage of over the K-e type models is that these types of models are able to describe flows in which the off-diagonal components of $R_{i j}$ are significant. Variable density extensions to these models also exist (BHR, 1987; Andronov, et al. 1982).

In an attempt to compensate for flows departing from spectral self-similarity, secondorder models that transport $R_{i j}$ and $\varepsilon$ at both the energy containing eddy scales and the energy cascade length scales are known as multi-scale models (Hanjalic, Launder \& Schiestel, 1980). These types of models represent the first indications that it is necessary to go to a two-point spectral model in order capture the dynamics of the turbulent kinetic energy spectrum.

Spectral models have been proposed for constant density homogeneous flow circumstances. In these models an exact but unclosed $R_{i j}$ transport equation is derived for two points in spaces as opposed to one (as in the discussions above). An $\varepsilon$ transport equation is no longer necessary in these models. Strategies for closing the triple correlations range from the relatively simple diffusion type approximation models of Leith (1967) and Kovasnay (1948) and the Eddy-Damped Quasi-Normal Markovian (EDQNM) model of Orsag (1970) to the more complicated Direct Interaction Approximation (DIA) family of closures (Kraichnan, 1958; 1959; 1964; 1965), the Random Coupling Model (RCM) (Kraichnan, 1961), and the Test Field Model (TFM) (Kraichnan, 1971; 1972). The work of Leith (1967), which follows the approach often used in radiation and neutron transport, and Kovasnay (1948) treat the transfer of energy in k-space as a local process while the rest of the previously mentioned models attempt to capture the nonlocal transfers of energy in k-space. Kraichnan (1966) showed quantitatively that a local approximation captures the essence of the physics. The BHRZ (1990) 
model for homogeneous constant density turbulence implements this local version of k-space dynamics .

Other two-point constant density turbulence transport models include those proposed by Cambon (1979), Cambon, et al. (1981), and Bertoglio (1982). Bertoglio and Jeandel (1987) use a spectral closure (EDQNM) for boundary layer calculations with constant density. Jeandel, Brison and Mathieu (1978) as well as Besnard et al. (1990) show how a spectral model can be integrated over $\mathrm{k}$-space by assuming self-similar form functions in $\mathrm{k}$-space. This integration reduces the spectral model to a single-point model, i.e., a $\mathrm{K}-\varepsilon$ type of model. Jeandel, et al. have applied their resulting single-point model to homogeneous and inhomogeneous turbulence and have had some success.

All of the spectral work mentioned to this point has been for constant density flows. Clark (1992) used the BHRZ spectral transport model to examine constant density anisotropic flows and the spectral behavior of return to isotropy. Clark and Spitz (1995) have developed a spectral transport model for homogeneous variable density flows. The work described herein addresses variable density inhomogeneous turbulence. 


\section{Configurational Definition of the Transported Variables Describing the Turbulent Mixing of Two Fluids}

\subsection{Introduction}

This chapter introduces and defines the flow field variables that are used in this study to describe the mixing of two immiscible fluids. These variables are used in the single-point BHR turbulence transport model discussed in Chapter 3, and the single-point two-fluid model discussed in Chapter 4. The two-point extension of the variables discussed herein are used in the spectral turbulence transport model developed in Chapter 5.

At this point we introduce the concept of an "ordered" and a "disordered" component of the flow. This concept fits in nicely with the ideas associated with the configurational variables discussed in this chapter. We associate the ordered component of the flow with the configurational variables discussed in this chapter. We have found that in keeping this conceptual detail of the flow in mind, it has aided us in the conceptual formulation of the spectral model of Chapter 5. For brevity we have elected to present the discussion on ordered and disordered components of the flow in Appendix C.

The next two chapters present two very different approaches for the theoretical analysis of fluid interpenetration across a nominal interface. One of these, based on a single-field approach (Chapter 3), is a single-point turbulence transport description, which we expand to a two-point (spectral) description in Chapter 5. The other is based on the equations for twofield flow (Chapter 4), from which we also use ideas and concepts in formulating the spectral transport model of Chapter 5.

The main thrust of this report is to develop a more firmly based foundation for turbulence transport calculations that are relevant for a broad scope of circumstances. The techniques for this endeavor are based on significant extensions of two-point spectral analysis to circumstances with large variations in properties from point to point throughout the fluid. This work is described in Chapter 5. However, in so doing, a firm understanding of the sin- 
gle-point modeling techniques for variable density turbulence must be established. Therefore, this chapter examines the relevant transported variables of a single-point turbulence transport model (BHR) and attaches physical meaning to these variables. The following two chapters then describe modeling techniques used to close transport equations for the variables discussed in this chapter.

\subsection{Description of the flow field variables}

In this study of variable-density turbulent mixing, it is necessary to define variables and concepts in addition to those used for the constant density case. In this section we describe the variables we work with in this report and use the configuration of a simplified onedimensional mixing layer between two incompressible immicible fluids to illustrate their definitions. For simplicity we use the linear stage of the Rayleigh-Taylor mixing layer as a basis for the example, but the concepts apply equally well to other mixing scenarios such as Kelvin-Helmholtz shears (Brown \& Roshko, 1974).

The conservation of mass and momentum equations are

$$
\frac{\partial \rho}{\partial t}+\frac{\partial \rho u_{n}}{\partial x_{n}}=0
$$

and

where

$$
\frac{\partial \rho u_{i}}{\partial t}+\frac{\partial \rho u_{i} u_{n}}{\partial x_{n}}=\frac{\partial \sigma_{i n}}{\partial x_{n}}+\rho g_{i}
$$

$$
\sigma_{\text {in }}=-\mathrm{p} \delta_{\text {in }}+\tau_{\text {in }} .
$$

The viscous stress is taken as

$$
\tau_{\text {in }}=\mu\left(\frac{\partial \mathrm{u}_{\mathrm{i}}}{\partial \mathrm{x}_{\mathrm{n}}}+\frac{\partial \mathrm{u}_{\mathrm{n}}}{\partial \mathrm{x}_{\mathrm{i}}}-\frac{2}{3} \delta_{\text {in }} \frac{\partial \mathrm{u}_{\ell}}{\partial \mathrm{x}_{\ell}}\right)
$$

where $\mu$ is the molecular viscosity.

We are interested in the ensemble averages of these equations. The definition of ensemble averaging that we use is analogous to that used by Hinze (1987) for $\mathrm{N}$ identical experiments. There exists a strong motivation to identify a velocity associated with the average mass flux, $\overline{\rho \mathbf{u}}$ (the overbar denotes an average), since this quantity occurs in both the 
averaged conservation of momentum equation as well as the averaged conservation of mass equation. If we apply the standard Reynolds decomposition, i.e., $\mathbf{u}=\overline{\mathbf{u}}+\mathbf{u}^{\prime}$ where $\overline{\mathbf{u}^{\prime}}=0$, to this momentum density, $\overrightarrow{\rho \mathbf{u}}$, we get

$$
\overline{\rho u_{i}}=\overline{\left(\bar{\rho}+\rho^{\prime}\right)\left(\bar{u}_{i}+u_{i}^{\prime}\right)}=\bar{\rho} \bar{u}_{i}+\overline{\rho^{\prime} u_{i}^{\prime}} .
$$

In order to identify a velocity that is responsible for fluxing the mass, we can use the Favre mass weighted velocity, $\tilde{\mathbf{u}}$, where $\mathbf{u}=\tilde{\mathbf{u}}+\mathbf{u}^{\prime \prime}$ with $\overline{\rho \mathbf{u}^{\prime \prime}}=0$. (Favre decomposition for the case of variable density flows contains many potential benefits over Reynolds decomposition, which incorporates many complexities into the averaged governing equations.) Thus we incorporate the mass weighted velocity,

into Eq. (2.2.5) to get

$$
\tilde{u}_{i}=\frac{\overline{\rho u_{i}}}{\bar{\rho}}
$$

$$
\tilde{u}_{i}=\bar{u}_{i}+\frac{\overline{\rho^{\prime} u_{i}^{\prime}}}{\bar{\rho}}
$$

We define another important variable here, namely the velocity a, associated with the net mass flux relative to the $\overline{\mathbf{u}}$ frame of reference. From Eq. $(2.2 .7)$ we can see that this velocity associated with this mass flux is just

$$
\mathbf{a}=\frac{\overline{\rho^{\prime} \mathbf{u}^{\prime}}}{\bar{\rho}} .
$$

This is a very important quantity as far as this study is concerned. It is this quantity that describes the fluxing of mass relative to the $\overline{\mathbf{u}}$ frame of reference, and this idea is quantified by the resulting expression,

$$
\tilde{\mathbf{u}}=\overline{\mathbf{u}}+\mathbf{a}
$$

There are two key concepts to identify in the above relationships. The first is the idea that from the quantity $\bar{\rho} \tilde{\mathbf{u}}$ we see that it is the velocity $\tilde{\mathbf{u}}$ that carries the mass flux, and the second is that it is this same velocity that carries the total momentum per unit volume. Here, it is instructive to note that a relationship between $\mathbf{a}$ and the mass weighted velocity 
fluctuation, $\mathbf{u}^{\prime \prime}$, can be established by inserting the Favre and Reynolds decomposition into Eq. (2.2.7) and ensemble averaging to obtain

$$
\mathbf{a}=-\overline{\mathbf{u}^{\prime \prime}} \text {. }
$$

(Note here that the ensemble average of the fluctuating mass weighted velocity is nonzero).

A transport equation for $\mathbf{a}$ is derived from the Navier-Stokes equations in the singlepoint model that is discussed in this Chapter 3. A transport equation for a is also formulated for the spectral model of Chapter 5.

We will now define the particular form of the Reynolds stress tensor, $R_{i j}$, that is used in this study. Decomposing the flow field variables of the mass and momentum equations into their mean and fluctuating parts followed by the ensemble averaging yields

and

$$
\frac{\partial \bar{\rho}}{\partial t}+\frac{\partial \bar{\rho} \tilde{u}_{n}}{\partial x_{n}}=0
$$

$$
\frac{\partial \bar{\rho} \tilde{u}_{i}}{\partial t}+\frac{\partial}{\partial x_{n}}\left(\bar{\rho} \tilde{u}_{i} \tilde{u}_{n}+R_{i n}\right)=\frac{\partial \bar{\sigma}_{\text {in }}}{\partial x_{i}}+\bar{\rho} g_{i}
$$

where, the Reynolds stress tensor, $R_{i n}$, in the momentum equation is

$$
R_{i j}=\overline{\rho u_{i}^{\prime \prime} u_{j}^{\prime \prime}}=\bar{\rho} \overline{u_{i}^{\prime} u_{j}^{\prime}}-\bar{\rho} a_{i} a_{j}+\overline{\rho^{\prime} u_{i}^{\prime} u_{j}^{\prime}} .
$$

Here the added complexity due to volume weighted variables as compared to mass weighted variables can be seen in the definition of $R_{\mathrm{ij}}$. It should also be noted that the process of Favre decomposition allows us to express both the momentum and mass equations in conservative form. These considerations of conservation also reinforce the motivation behind the identification of this generalized Reynolds stress tensor.

With the above expression for $R_{\mathrm{ij}}$, it is instructive to look at the expression for the kinetic energy per unit volume, $\bar{\rho} \mathrm{KE}$, hereafter referred to as just the kinetic energy of the flow. By definition, the total kinetic energy of a flow is

$$
\bar{\rho} \mathrm{KE}=\frac{1}{2} \overline{\mathrm{pu}_{\mathrm{n}} \mathrm{u}_{\mathrm{n}}} .
$$


The total kinetic energy receives contributions from the mean flow associated with the center of mass as well as the turbulent component of the flow. To demonstrate this we substitute the mass-weighted decomposition of the velocity field, $\mathbf{u}=\tilde{\mathbf{u}}+\mathbf{u}^{\prime \prime}$, into Eq. (2.2.13),

$$
\bar{\rho} \mathrm{KE}=\frac{1}{2} \overline{\rho\left(\tilde{\mathrm{u}}_{\mathrm{n}}+\mathrm{u}_{\mathrm{n}}^{\prime \prime}\right)\left(\tilde{\mathrm{u}}_{\mathrm{n}}+\mathrm{u}_{\mathrm{n}}^{\prime \prime}\right)},
$$

and, upon ensemble averaging, the expression for the total kinetic energy becomes

$$
\bar{\rho} K E=\frac{1}{2}\left[\bar{\rho} \tilde{u}_{\mathfrak{n}} \tilde{u}_{n}+2 \tilde{u}_{n} \overline{\rho u_{n}^{\prime \prime}}+\overline{\rho u_{n}^{\prime \prime} u_{n}^{\prime \prime}}\right] .
$$

Since (by definition) $\overline{\rho u^{\prime \prime}}=0$, the expression for the kinetic energy simplifies to

or,

$$
\bar{\rho} K E=\frac{1}{2}\left[\bar{\rho} \tilde{u}_{n} \tilde{u}_{n}+\overline{\rho u_{n}^{\prime \prime} u_{n}^{\prime \prime}}\right],
$$

$$
\bar{\rho} K E=\frac{1}{2}\left[\bar{\rho} \tilde{u}_{\mathrm{n}} \tilde{\mathrm{u}}_{\mathrm{n}}+\mathrm{R}_{\mathrm{nn}}\right] .
$$

From this expression it is easy to identify the only two contributions to the total kinetic energy of this particular turbulent flow. Here $R_{n n}$ represents the total kinetic energy per unit volume that is not associated with the movement of the center of mass, i.e., it represents the turbulent contributions only.

Two more important quantities to be identified here, which appear in the transport equation for $\mathbf{a}$, are the density-specific volume correlation, $b$, and the density-density self correlation, B, where

and,

$$
b=-\overline{\rho^{\prime} v^{\prime}}
$$

The specific volume, $\mathrm{v}$, is defined by

$$
B=\overline{\rho^{\prime} \rho^{\prime}} .
$$

$$
\mathrm{v}=\frac{1}{\rho} \text {. }
$$

Here for the idealization of a smooth interface between two interpenetrating liquids, we attach physical significance to the variables used in this study by examining this highly idealized mixing interface. The following figure used to describe the mixing situation is highly idealized, but nonetheless, it serves a useful purpose. 


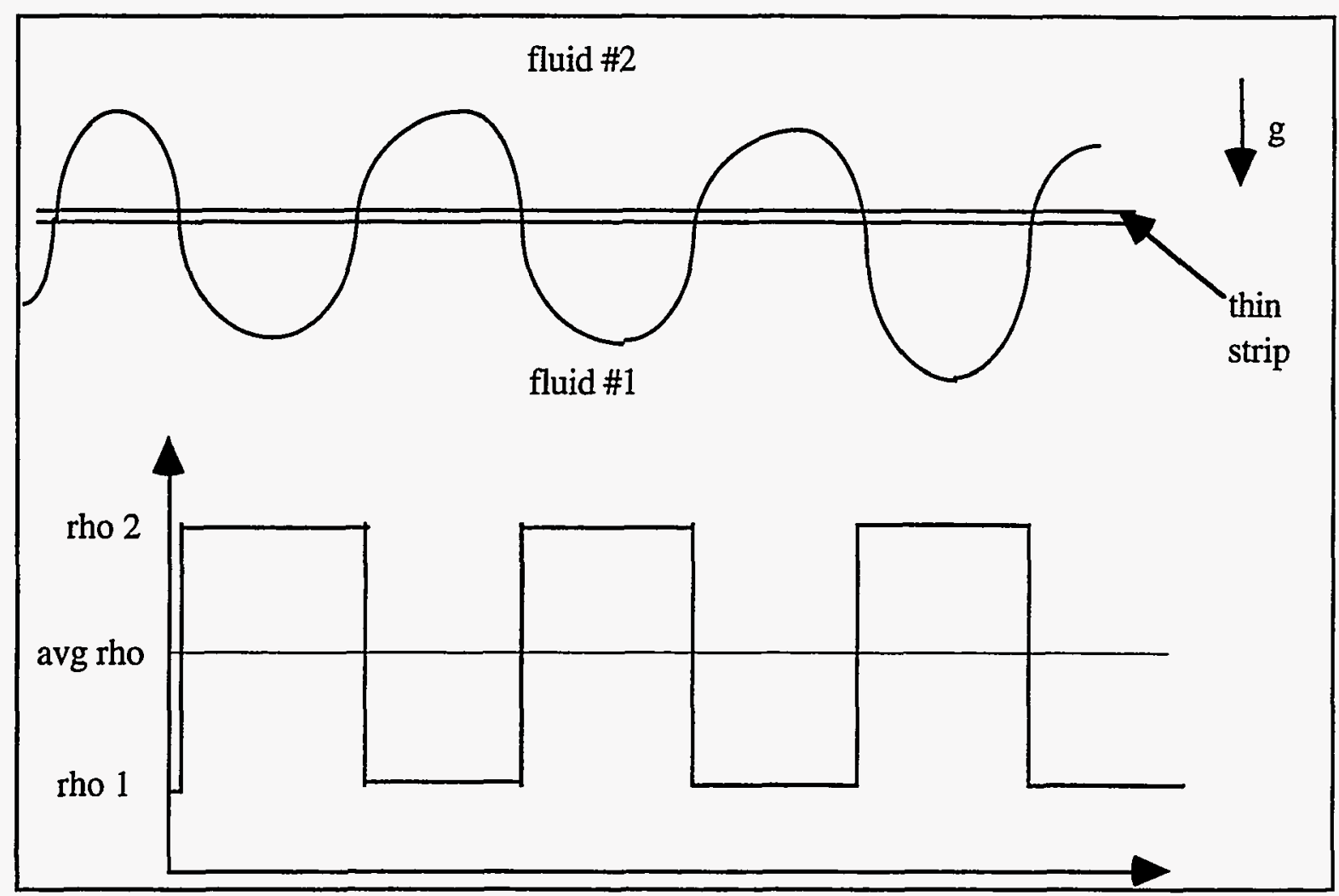

Figure 2.2.1. Idealized schematic of an interface between two interpenetrating fluids (top) with the corresponding density profile (bottom) found in the thin horizontal strip through the mixing layer.

Given the mixing interfacial configuration of Fig. 2.2.1, both materials are in the liquid phase in order to simplify this explanation. In this figure a heavy fluid is situated over a lighter fluid with gravity pointing down. The fluids are constrained to be at rest far above and below the interface. Thus a vertical pressure gradient is created by the presence of gravity, and the configuration is unstable. The perturbed interface is shown with a thin strip drawn horizontally through it. The bottom half of the figure shows the spatial density distribution found within the strip. For our purpose, imagine this slice of the interface extending forever in the horizontal direction. A spatial average across the strip can be thought of as an ensemble average of a large number of experiments describing fluid mixing.

To identify average values of density, velocity and other variables it is instructive to take a strip average as shown in the above configuration. As we traverse the strip, it can be seen that there will exist a fraction of circumstance, $\alpha_{1}$, when we are in fluid 1 and a differ- 
ent fraction of circumstance, $\alpha_{2}$, when we are in fluid 2. From this process it follows that we can define a volume averaged density, $\bar{\rho}$, and a volume average velocity, $\overline{\mathbf{u}}$, for the strip as

and

$$
\bar{\rho}=\alpha_{1} \rho_{1}+\alpha_{2} \rho_{2} \text {, }
$$

where,

$$
\overline{\mathbf{u}}=\alpha_{1} \mathbf{u}_{1}+\alpha_{2} \mathbf{u}_{2},
$$

$$
\alpha_{1}+\alpha_{2}=1
$$

Here $\alpha_{1}$, and $\alpha_{2}$, are the volume fractions, $\rho_{1}$ and $\rho_{2}$ are the microscopic densities of fluid 1 and 2 , respectively, while $\mathbf{u}_{1}$ and $\mathbf{u}_{2}$ are the separately averaged velocities of fluid 1 and fluid 2, respectively found in the thin strip. Note that for the special case when $\mathbf{u}_{1}=-\mathbf{u}_{2}$, at the center of the mixing layer where $\alpha_{1}=\alpha_{2}=0.5$, then the condition of incompressibility ensures the condition $\overline{\mathbf{u}}=0$, for the whole mixing layer. It should also be noted that these two averages can be generalized for a mixture involving a large number, $n$, of fluids as

$$
\begin{aligned}
& \bar{\rho}=\sum_{i=1}^{n} \alpha_{i} \rho_{i}, \\
& \overline{\mathbf{u}}=\sum_{i=1}^{n} \alpha_{i} \mathbf{u}_{i},
\end{aligned}
$$

and,

$$
\sum_{i=1}^{n} \alpha_{i}=1
$$

In the same light that we define a volume fraction for fluids 1 and 2 , we also define mass fractions, $c_{1}$ and $c_{2}$, of fluids one and two such that

Here

$$
c_{1}+c_{2}=1
$$

and,

$$
\mathrm{m}_{1}=\rho_{1} \alpha_{1} \delta \mathrm{V}=\mathrm{c}_{1} \mathrm{M}
$$

$$
\mathrm{m}_{2}=\rho_{2} \alpha_{2} \delta \mathrm{V}=\mathrm{c}_{2} \mathrm{M} \text {, }
$$

where $M$ is the total mass of all fluids in a $\delta \mathrm{V}$ volume segment of the strip. Thus $c_{1} \bar{\rho}=\alpha_{1} p_{1}$ and $c_{2} \bar{\rho}=\alpha_{2} \rho_{2}$ 
It is very useful to describe the momentum of the strip of fluid as the product of the overall density times the velocity, $\tilde{\mathbf{u}}$. If one observes the flow field in the frame of reference in which the strip is at rest, the volumetric flux through the strip will be zero due to incompressibility. For the incompressible case of two non-equal fluids mixing, $\rho_{1}$ is different from $\rho_{2}$, and there is a net mass flux across the strip. This mass fluxing velocity is clearly not $\overline{\mathbf{u}}$, since $\overline{\mathbf{u}}$ is zero in this case. Upon careful examination of the strip, we realize that the momentum per unit volume for fluid one and two is $\rho_{1} \mathbf{u}_{1}$ and $\rho_{2} \mathbf{u}_{2}$, respectively, and the total momentum density of the strip is just $\alpha_{1} \rho_{1} \mathbf{u}_{1}+\alpha_{2} \rho_{2} \mathbf{u}_{2}$. This allows for the very useful and intuitive identification for the mass-fluxing velocity of the strip, such that

$$
\bar{\rho} \tilde{\mathbf{u}}=\rho_{1} \alpha_{1} \mathbf{u}_{1}+\rho_{2} \alpha_{2} \mathbf{u}_{2}
$$

The relationship provides a bridge between the two-fluid flow variables and the total momentum of a single combined field.

We can also determine equivalent configurational expressions for $a_{i}$, the velocity of the net mass flux, and $\mathrm{R}_{\mathrm{ij}}$, the Reynolds stress tensor. While horizontally traversing the strip of fluid in Fig. 2.2.1, fluctuating quantities can be expressed as deviations of that field away from the overall averages of the flow in the strip. That is to say, the density fluctuation, $\rho^{\prime}$, can be expressed as either $\rho_{1}-\bar{\rho}$ or $\rho_{2}-\bar{\rho}$ depending on whether we are in fluid one or fluid two. Similar expressions can be formulated for the following two representations of the fluctuating velocity field: (1) the quantities $\mathbf{u}_{1}-\overline{\mathbf{u}}$ or $\mathbf{u}_{2}-\overline{\mathbf{u}}$, depending on which field we are in, for the fluctuating velocity $\mathbf{u}^{\prime}$, or (2) the quantities $\mathbf{u}_{1}-\tilde{\mathbf{u}}$ or $\mathbf{u}_{2}-\tilde{\mathbf{u}}$, again depending on which field we are in, for the fluctuating velocity $\mathbf{u}^{\prime \prime}$. As we horizontally traverse the strip we are in fluid one $\alpha_{1}$ fraction of the traverse and fluid two $\alpha_{2}$ fraction of the traverse.

Thus, upon substitution of these expressions into Eq. (2.2.8) we find that

$$
a_{i}=\frac{\alpha_{1}\left(\rho_{1}-\bar{\rho}\right)\left(u_{1}-\bar{u}\right)_{i}+\alpha_{2}\left(\rho_{2}-\bar{\rho}\right)\left(u_{2}-\bar{u}\right)_{i}}{\bar{\rho}}
$$


An exercise in algebra leads to the following configurational formulation for the velocity of the net mass flux, $\mathrm{a}_{\mathrm{i}}$ :

$$
a_{i}=\frac{\alpha_{1} \alpha_{2}\left(\rho_{1}-\rho_{2}\right)\left(u_{1}-u_{2}\right)_{i}}{\bar{\rho}} .
$$

From the bottom configuration of Fig. 2.2.1 it can be seen that the density changes as we transverse the thin strip. These fluctuations away from the average value of density are once again defined as $\left(\rho_{1}-\bar{\rho}\right)$ or $\left(\rho_{2}-\bar{\rho}\right)$, depending upon which fluid we are in. Upon substitution of the expressions for the fluctuating quantities into the specific volume-density correlation, $b$, we get

$$
\mathrm{b}=-\left[\alpha_{1}\left(\rho_{1}-\bar{\rho}\right)\left(\frac{1}{\rho_{1}}-\overline{\mathrm{v}}\right)+\alpha_{2}\left(\rho_{2}-\bar{\rho}\right)\left(\frac{1}{\rho_{2}}-\overline{\mathrm{v}}\right)\right] .
$$

An exercise in algebra leads to the following configurational expression for $b$ :

$$
b=\frac{\alpha_{1} \alpha_{2}\left(\rho_{1}-\rho_{2}\right)^{2}}{\rho_{1} \rho_{2}} .
$$

Likewise, a similar expression can be derived for the density-density self-correlation, B.

Substitution of the expressions for the fluctuating densities into the definition of $B$, results in

$$
B=\alpha_{1}\left(\rho_{1}-\bar{\rho}\right)\left(\rho_{1}-\bar{\rho}\right)+\alpha_{2}\left(\rho_{2}-\bar{\rho}\right)\left(\rho_{2}-\bar{\rho}\right) .
$$

Once again, after some algebra, the expression for the density-density self correlation within the thin strip of Fig. 2.2.1 becomes

$$
B=\alpha_{1} \alpha_{2}\left(p_{1}-p_{2}\right)^{2} .
$$

In this study, we work with $b$ as one of our primary variables.

Substitution of the configurational expressions for the mass weighted fluctuating velocities into Eq. (2.2.12) results in a similar configurational expression (denoted by the superscript, c) for $\mathrm{R}_{\mathrm{ij}}$, namely,

$$
R_{i j}^{c}=\alpha_{1} \rho_{1}\left(u_{1}-\tilde{u}\right)_{i}\left(u_{1}-\tilde{u}\right)_{j}+\alpha_{2} \rho_{2}\left(u_{2}-\tilde{u}\right)_{i}\left(u_{2}-\tilde{u}\right)_{j},
$$


which, after some algebraic manipulation, becomes

$$
R_{i j}^{c}=\frac{\alpha_{1} \alpha_{2} \rho_{1} \rho_{2}\left(u_{1}-u_{2}\right)_{i}\left(u_{1}-u_{2}\right)_{j}}{\bar{\rho}}=\frac{a_{i} a_{j} \bar{\rho}}{b} .
$$

This expression for the Reynolds stress also allows for the description of the turbulent kinetic energy per unit mass, $K$, via the two field variables, where

$$
\mathrm{K}^{\mathrm{c}}=\frac{\mathrm{R}_{\mathrm{nn}}^{\mathrm{c}}}{2 \overline{\mathrm{\rho}}}
$$

namely,

$$
K^{c}=\frac{\alpha_{1} \alpha_{2} \rho_{1} \rho_{2}\left(u_{1}-u_{2}\right)_{n}\left(u_{1}-u_{2}\right)_{n}}{2 \bar{\rho}^{2}}=\frac{a_{n} a_{n}}{2 b} .
$$

Here, the repeated indices are summed in the usual fashion. Because $\mathbf{u}_{1}$ and $\mathbf{u}_{2}$ are average velocities within each fluid, the $R_{i j}$ and $K$ calculated in this manner carry none of the effects of the intrafluid fluctuations. We are, therefore, led to the idea that these configurational descriptions carry, in some sense, only the effects of the ordered part of the interpenetration. To be more illustrative of the idea, consider the decomposition of the kinetic energy in Eq. (2.2.15) to be further subdivided into that part which represents the fluctuations from blob to blob and the part which represents the fluctuations of velocity within fluid 1 and fluid 2 .

This suggests an identification of the ordered and disordered parts of the turbulent kinetic energy such that Eq. (2.2.39) is appropriately recognized as the ordered part and the disordered part of the turbulent kinetic energy per unit mass, $K_{\text {diss }}$, is represented by the expression

$$
K_{\text {diss }}=\frac{R_{n n}}{2 \bar{\rho}}-\frac{R_{n n}^{c}}{2 \bar{\rho}}
$$

In this section definitions of important variables and their associated configurational basis that are to be used for the rest of this report have been given. 


\subsection{Summary of the flow field variables}

In this chapter we discussed the variables used for the rest of this study. The variables are derived from a configurational point of view for a two-fluid flow configuration. This chapter has been included to give a physical feel for the significance of the variables that are transported in the spectral turbulence transport model developed in Chapter 5 and aiso the single-point discussions of Chapters 3 and 4.

The variables in this chapter were described in a single-point context to give an intuitive feel for the physics that we wish to represent with each of them. These are all important concepts when one thinks about the ideas of mass-flux and density correlations as being the primary sources for variable density flows. 


\section{Single-Point Turbulence Transport Modeling}

\subsection{Introduction}

This chapter presents a single-point turbulence transport approach for the theoretical analysis of fluid interpenetration across a nominal interface. This approach, based on a single-field description, is a single-point turbulence transport model (BHR), which we expand to a two-point (spectral) description in Chapter 5. The purpose of this chapter, along with Chapter 4, is to present some of the strategies that are used as a basis for the formulation of the spectral approach to the theoretical analysis of fluid interpenetration that is presented in Chapter 5.

Here, when we refer to the single-point turbulence transport model, we are referring to a model developed directly from the Navier-Stokes equations which treats the entire flow field at a single point in space. The flow field variables are decomposed into mean and fluctuating parts, and through the process of ensemble averaging a mean flow field and a turbulent flow field can then be identified. This differs from the two-field approach in that the two-field flow model discussed in Chapter 4 follows the standard convention of keeping two separate velocity fields and the mixture fraction at every point for a nonturbulent highly dispersed flow. Both models come from the Navier-Stokes equations and require much modeling, incorporate much empiricism, and neither is capable of describing all the circumstances that are currently of interest.

There are many scenarios that can be described in the variable density context or the two-field flow context. An example for each is, respectively, (1) two interpenetrating fluids, and, (2) widely dispersed marbles falling through a fluid. These two examples are given only to represent the extreme cases which might be described either by the single-point turbulence transport equations for a variable density fluid or the two-field flow equations. The flow that

this study is concerned with is the case for two immiscible interpenetrating fluids under the 
influence of an acceleration with vanishing viscosity or the case of blobs of fine particles in a fluid mixing into the pure fluid. In all cases, the issue of changing flow scales is of extreme significance. A model must be capable of capturing the experimentally observed flow scale growth. For the case of two interpenetrating immiscible fluids, the quadratic growth of bubbles can be observed, and for the case of particulate mixing within a pure fluid the coalescence or fragmentation of particle-liquid blobs can be observed; both represent examples of scale growth.

In this chapter we present a discussion of single-point turbulence transport modeling for variable density mixing of two fluids. The guidance one can obtain from two-field flow when attempting to model single-point turbulence transport equations will be discussed. We also investigate the various modeling techniques used in the single-point theory. In the literature (Polyonov, 1989a, 1989b \& 1989c; ) it is demonstrated that current single-point transport models can be adjusted to agree rather well with a restricted subset of interesting problems of fluid-interface instability and turbulent mix, and the single-point transport models give qualitatively useful results for a somewhat greater set of problems. These agreements with experiments and numerical simulations come at the expense of requiring inconsistencies in the formulations, like the elimination of terms that seem to arise properly from the modeling but are deleterious when tested in calculations. In this chapter it will also be shown that there are major, fundamental elements of formulation that are missing from the current single-point transport formulations. These elements cannot be found with the guidance of two-field flow formulations, because they are associated with the changing scales of fluctuation that twofield flow theories are incapable of describing without empirical supplementation.

Thus the purpose of this chapter is two-fold. It demonstrates a basis for partial validity of current single-point transport modeling and indicates some major directions for improvements that can be made to increase the breadth of that validity. In this chapter we address many of the philosophical shortcomings of single-point turbulence transport theories to 
motivate the need for the enhancements of a spectral theory. It establishes a perspective for the spectral developments and comparisons discussed in Chapter 5.

\subsection{Single-point turbulence transport equations}

There are many single-point theories for constant density turbulence. As stated in the literature survey of Chapter 1 , these theories range from the very simple zero-equation models to the more complicated multi-equation models. For two-fluid mixing problems, there are many fewer single-point transport models, and these consist mainly of variations of the model developed by the authors Besnard, Harlow and Rauenzahn (Besnard et al. 1992), and the models developed by Nikiforov (1991) and Launder, et al. (1975). Jones and Launder (1972) introduced a two-equation, K- $\varepsilon$, single-point turbulence transport model for constant density recirculating flows. They make use of gradient-diffusion models for the turbulent flux of scalar quantities. Kuo (1986) proposes an extension of this model to account for variable density but points out that these extensions have not been tested and, according to Jones and Whitelaw (1982), more than likely will not be tested in the near future. The current status of variable density turbulence transport research is adequately described in the rest of this section. A more complete overview (up to 1980) of the current state of variable density turbulence transport models is given by Jones (1980). This was presented as part of a lecture series on turbulent flows.

The purpose of this section is to establish an overview on the current state of singlepoint theory as applied to variable density problem of interface mixing. Since the emphasis of this report is on the extension to two-point spectral turbulence theory, this discussion emphasizes the single-point model (BHR) used as a basis for the spectral model developed in Chapter 5 and indicates some of the deficiencies of the BHR single-point model in terms of scale considerations. This serves as an indicator of current modeling techniques used for variable density flows. 
The BHR single-point turbulence transport model is applicable to multimaterial compressible turbulent flows. In this section, in compliance with the strategy of BHR, a set of transport equations for second order correlations, namely $\mathbf{a}, \mathrm{b}$, and $\mathrm{R}_{\mathrm{ij}}$, appropriate to variable-density turbulence as described earlier in Chapter 2 are derived from the Navier-Stokes equations. Gradient-type diffusion approximations developed from mean flow variables are applied to some but not all of the other second and higher order correlations to properly close the system of model equations. The BHR model is developed for compressible turbulent flows whose fluctuating velocities are far subsonic. This scenario is very common in nature.

In Appendix A, we show that this single-point turbulence transport model (BHR) conserves energy. This is an important and necessary condition for a credible transport model. This conservation of energy consideration is also very important in the formulation of the spectral model of Chapter 5. It can be shown that the spectral model of Chapter 5 , when fully integrated in $\mathrm{k}$-space, also conserves energy.

In this section a derivation for the transport equations of the BHR single-point turbulence transport model is presented to point out some of the modeling techniques for variabledensity single-point theory. The modeling techniques associated with this model are used as a basis on which to build the spectral model for variable-density turbulence presented in Chapter 5.

The BHR model allows for the mean flow to be compressible, and only the turbulent velocity field is subsonic. For the purposes of this report we assume that the entire flow field is divergence-free, so that $\nabla \cdot \overline{\mathbf{u}}=0$ and $\nabla \cdot \mathbf{u}^{\prime}=0$. Since our applications are to the turbulent mixing of incompressible fluids the internal energy equation, equation of state, and the species transport equation are not required here. For the incompressible case involving only two species, a species transport equation is derived directly from the conservation of mass equation. The different types of modeling used to close the system are discussed. The derivation rests on the assumptions that the Navier-Stokes equations completely describe a 
fluid flow field in which there can be large discontinuities in density and that this flow field can be separated into mean and fluctuating components.

The equations for the conservation of mass and momentum, restated from Chapter 2 here for convenience, are

and

$$
\frac{\partial \rho}{\partial t}+\frac{\partial \rho u_{n}}{\partial x_{n}}=0
$$

where

$$
\frac{\partial \rho u_{i}}{\partial t}+\frac{\partial \rho u_{i} u_{n}}{\partial x_{n}}=\frac{\partial \sigma_{i n}}{\partial x_{n}}+\rho g_{i} \text {, }
$$

$$
\sigma_{\mathrm{in}}=-\mathrm{p} \delta_{\mathrm{in}}+\mu\left(\frac{\partial \mathrm{u}_{\mathrm{i}}}{\partial \mathrm{x}_{\mathrm{n}}}+\frac{\partial \mathrm{u}_{\mathrm{n}}}{\partial \mathrm{x}_{\mathrm{i}}}-\frac{2}{3} \delta_{\mathrm{in}} \frac{\partial \mathrm{u}_{\ell}}{\partial \mathrm{x}_{\ell}}\right) .
$$

For this study, the fluids are taken as immiscible; therefore, no account must be taken for molecular diffusion. Also, no account is taken of phase transitions, and molecular viscosity, $\mu$, is considered to be vanishingly small, since this effect is negligible for the case of the Rayleigh-Taylor instability (Youngs, 1984). The Reynolds decomposition, as discussed in Chapter 2, is applied to these equations in the conventional manner by decomposing the flow field variables into their mean and fluctuating components. Once these decompositions are inserted into the above conservation relations, the resulting equations are ensemble averaged. At this point we make use of the Favre decomposition allowing for a convenient description for the momentum of the flow field. As shown in Chapter 2, the Favre decomposition separates the velocity field into the mass weighted ensemble averaged component and the fluctuating component.

After introducing the above decompositions, ensemble averaging, and applying the applicable relations from Chapter 2, we get the following conservation relations:

and

$$
\frac{\partial \bar{\rho} \tilde{u}_{i}}{\partial t}+\frac{\partial}{\partial x_{n}}\left(\bar{\rho} \tilde{u}_{i} \tilde{u}_{n}+R_{i n}\right)=\frac{\partial \bar{p}}{\partial x_{i}}+\bar{\rho} g_{i},
$$

$$
\frac{\partial \bar{\rho}}{\partial t}+\frac{\partial \bar{\rho} \tilde{u}_{n}}{\partial x_{n}}=0
$$


Notice here that the Favre decomposition process allows us to express the mass and the momentum equations in conservative form and allows for the simple identification of the generalized Reynolds stress, $\overline{\rho \mathbf{u}^{\prime \prime} \mathbf{u}^{\prime \prime}}$. Here it is observed that, when written in this form, the Reynolds stress represents that part of the flow which is not mean flow.

In the BHR model a transport equation is derived for $\mathrm{R}_{\mathrm{ij}}$. This transport equation is first derived in rigorous but unclosed form from the Navier-Stokes equations. To construct a transport equation for $\mathrm{R}_{\mathrm{ij}}$, the variables in the Navier-Stokes equations are decomposed into mean and fluctuating parts and transport equations are derived for $\rho \mathbf{u}^{\prime \prime}$ and $\mathbf{u}^{\prime \prime}$. The transport equation for $\rho \mathbf{u}^{\prime \prime}$ is multiplied by $\mathbf{u}^{\prime \prime}$, and the transport equation for $\mathbf{u}^{\prime \prime}$ is multiplied by $\rho u^{\prime \prime}$. The resulting two transport equations are added together and ensemble averaged so as to create a transport equation for $R_{i j}$. The resulting unmodeled transport equation for the Reynolds stress, $R_{i j}$ is

$$
\begin{aligned}
\frac{\partial R_{i j}}{\partial t}+\frac{\partial R_{i j} \tilde{u}_{n}}{\partial x_{n}}+ & R_{i n} \frac{\partial \tilde{u}_{j}}{\partial x_{n}}+R_{n j} \frac{\partial \tilde{u}_{i}}{\partial x_{n}}+\frac{\partial R_{n i j}}{\partial x_{n}} \\
& =\left(a_{j} \frac{\partial \bar{p}}{\partial x_{i}}+a_{i} \frac{\partial \bar{p}}{\partial x_{j}}\right)-\frac{\partial \overline{u_{j}^{\prime} p^{\prime}}}{\partial x_{i}}-\frac{\partial \overline{u_{i}^{\prime} p^{\prime}}}{\partial x_{j}}+\overline{p^{\prime} \frac{\partial u_{j}^{\prime}}{\partial x_{i}}+\overline{p^{\prime}} \frac{\partial u_{i}^{\prime}}{\partial x_{j}}} .
\end{aligned}
$$

The triple correlation $R_{n i j}$ is defined as $\overline{\rho u_{n}^{\prime \prime} u_{i}^{\prime \prime \prime} u_{j}^{\prime \prime}}$. Notice a paradox that emerges from Eq. (3.2.6) when molecular viscosity is neglected. There are no dissipation terms. Yet, it is well known that all forms of turbulence decay, even at very high Reynolds numbers, i.e., where the molecular viscosity is practically zero. This is the identification of one of the motivating reasons to construct a two-point spectral model. As will be shown in Chapter 5 the spectral model is capable of producing terms that, when spectrally integrated, represent a physically plausible means of dissipation. In the spectral model of Chapter 5 the dissipation rate is determined by the rate of inertial transfer and is insensitive to the magnitude of viscosity. The rate of inertial transfer is scale dependent, and the single-point formulation of Eq. (3.2.6) is incapable of providing any meaningful description of a scale for $\mathrm{R}_{\mathrm{ij}}$. 
To model the triple correlation, $R_{n i j}$, of Eq. (3.2.6), we use the relation $u_{n}^{\prime \prime}=u_{n}^{\prime}-a_{n}$, to re-express the gradient of this correlation as

$$
\frac{\partial}{\partial x_{n}} \overline{\rho u_{n}^{\prime \prime} u_{i}^{\prime \prime} u_{j}^{\prime \prime}}=\frac{\partial}{\partial x_{n}} \overline{\rho u_{n}^{\prime} u_{i}^{\prime u_{j}^{\prime \prime}}}-\frac{\partial}{\partial x_{n}} a_{n} R_{i j}
$$

This procedure produces a triple correlation that must be modeled and a term that can remain unmodeled. The following gradient approximation used by Daly and Harlow (1970) is used to model the triple correlation and the conservative part of the pressure-velocity correlations:

$$
\overline{\rho u_{n}^{\prime} u_{i}^{\prime \prime} u_{j}^{\prime \prime}}=-C_{D R} \frac{K}{\varepsilon}\left[R_{n \ell} \frac{\partial}{\partial x_{\ell}}\left(\frac{R_{i j}}{\bar{\rho}}\right)\right] .
$$

Here $C_{D R}$ is a constant, $K$ is the turbulent kinetic energy, and $\varepsilon$ is the decay of $K$. See Eq. (3.2.13) and Eq. (3.2.14) for transport equations for $\mathrm{K}$ and $\varepsilon$. Although numerous permutations of indices are suggested by the symmetries of $\mathrm{R}_{\mathrm{nij}}$, we follow the procedure of using only the part that is directly proportional to the gradient of $R_{i j}$ itself.

The modeling of the pressure-velocity gradient correlations is divided into two parts: the part that is coupled directly to the mean flow gradient, and the part that is modeled as a return to isotropy due to turbulent eddy interactions. This is expressed as

$$
-\mathrm{C}_{\mathrm{IR}} \frac{\varepsilon}{\mathrm{K}}\left(\mathrm{R}_{\mathrm{ij}}-\frac{1}{3} \delta_{\mathrm{ij}} \mathrm{R}_{\mathrm{nn}}\right) .
$$

The part that is coupled directly to the mean flow is modeled as a direct extension of the model developed by Launder, Reece, \& Rodi (1975) for the constant density case, namely,

$$
C_{2 R}\left(R_{i n} \frac{\partial \tilde{u}_{j}}{\partial x_{n}}+R_{j n} \frac{\partial \tilde{u}_{i}}{\partial x_{n}}-\frac{2}{3} \delta_{i j} R_{m n} \frac{\partial \tilde{u}_{m}}{\partial x_{n}}\right)
$$

A type of dissipation must be included to describe the decay of turbulence. As pointed out by previous authors (Launder, et al. 1975; BHR, 1987), for constant-density turbulence, the decay of $R_{i j}$ is well represented by the addition of the term,

$$
-\frac{2}{3} \bar{\rho} \varepsilon \delta_{\mathrm{ij}}
$$


but more generally we believe that there could be significant off-diagonal contributions to this decay term. In Chapter 5 we address these issues from spectral derivations.

With the above modeling, the transport equation for $R_{i j}$ becomes

$$
\begin{aligned}
\frac{\partial R_{i j}}{\partial t}+ & \frac{\partial R_{i j} \bar{u}_{n}}{\partial x_{n}}+R_{i n} \frac{\partial \tilde{u}_{j}}{\partial x_{n}}+R_{j n} \frac{\partial \tilde{u}_{i}}{\partial x_{n}} \\
& =\left(a_{i} \frac{\partial \bar{p}}{\partial x_{j}}+a_{j} \frac{\partial \bar{p}}{\partial x_{i}}\right)+C_{D R} \frac{\partial}{\partial x_{m}}\left[\frac{K}{\varepsilon} R_{m n} \frac{\partial}{\partial x_{n}}\left(\frac{R_{i j}}{\bar{\rho}}\right)\right] \\
-C_{1 R} & \frac{\varepsilon}{K}\left(R_{i j}-\frac{1}{3} \delta_{i j} R_{n n}\right)-C_{2 R}\left(R_{i n} \frac{\partial \tilde{u}_{j}}{\partial x_{n}}+R_{j n} \frac{\partial \tilde{u}_{i}}{\partial x_{n}}-\frac{2}{3} \delta_{i j} R_{m n} \frac{\partial \tilde{u}_{m}}{\partial x_{n}}\right)-\frac{2}{3} \delta_{i j} \bar{\rho} \varepsilon .
\end{aligned}
$$

Instead of transporting the full Reynolds stress tensor $\mathrm{R}_{\mathrm{ij}}$, a transport equation for the turbulent kinetic energy per unit mass, $\mathrm{K}$, is sometimes used and is derived with the following definition:

$$
\bar{\rho} \mathrm{K}=\frac{1}{2} R_{\mathrm{nn}} .
$$

Contraction of Eq. (3.2.11) results in

$$
\frac{\partial \bar{\rho} K}{\partial t}+\frac{\partial \bar{\rho} K \tilde{u}_{n}}{\partial x_{n}}+R_{n m} \frac{\partial \tilde{u}_{m}}{\partial x_{n}}=a_{n} \frac{\partial \bar{p}}{\partial x_{n}}+\frac{\partial}{\partial x_{n}}\left(\bar{\rho} v_{t} \frac{\partial K}{\partial x_{n}}\right)-\bar{\rho} \varepsilon
$$

From the transport equation of the turbulent kinetic energy, dimensional arguments can be used to establish the following transport equation for the decay of the turbulent kinetic energy, $\varepsilon$ :

$$
\frac{\partial \bar{\rho} \varepsilon}{\partial t}+\frac{\partial \bar{\rho} \varepsilon \tilde{u}_{n}}{\partial x_{n}}+C_{1 \varepsilon} \frac{\varepsilon}{K} R_{n m} \frac{\partial \tilde{u}_{m}}{\partial x_{n}}=C_{3 \varepsilon} \frac{\varepsilon}{K} a_{n} \frac{\partial \bar{p}}{\partial x_{n}}+C_{D \varepsilon} \frac{\partial}{\partial x_{n}}\left(\frac{\bar{\rho} K^{2}}{\varepsilon} \frac{\partial \varepsilon}{\partial x_{n}}\right)-C_{2 \varepsilon} \frac{\varepsilon^{2} \bar{\rho}}{K}
$$

Some investigators prefer to combine the $\mathrm{K}$ and $\varepsilon$ equations, discarding some terms along the way, in order to transport $\mathrm{K} \sqrt{\mathrm{K}} / \varepsilon$, which has the dimensions of length, and is here called $\mathrm{S}$. The resulting equation for the length scale $\mathrm{S}$ is

$$
\begin{aligned}
& \frac{\partial \rho S}{\partial t}+\frac{\partial \rho S u_{n}}{\partial x_{n}}= \\
& \frac{\partial}{\partial x_{n}}\left(\frac{\rho v_{t}}{\sigma_{\varepsilon}} \frac{\partial S}{\partial x_{n}}\right)+C_{s 3} \rho S \frac{\partial u_{n}}{\partial x_{n}}+\frac{S}{K}\left[C_{s 4} a_{n} \frac{\partial p}{\partial x_{n}}-C_{s 1} R_{i j} \frac{\partial u_{i}}{\partial x_{n}}\right]-C_{s 2} \rho \sqrt{K} .
\end{aligned}
$$


Equations (3.2.14) and (3.2.15) provide additional motivation to formulate a spectral transport model. Both equations are derived heuristically on the grounds of dimensional arguments. A wonderful feature of the spectral equations is the alleviation of this need for a transport equation describing the dissipation of turbulent kinetic energy, $\mathrm{K}$. This concept of dissipation is handled with a spectral model in terms describing the cascade of energy from the large scale structures to the smaller scale structures, which is a feature that the singlepoint equations are incapable of describing. This concept is tied much more closely with physical reality than Eq. (3.2.14). The only time that Eq. (3.2.14) gives a valid representation of the dissipation of turbulent kinetic energy is when the flow is in this so-called spectral equilibrium. That is to say, if the flux of energy from the large scales of the flow is identical to the flux of energy being converted to heat through viscous processes, then the flow is in spectral equilibrium, and the single-point dissipation equation is valid. But, once this equilibrium is ruined due to a transient in the driver of the flow, then Eq. (3.2.14) is no longer valid, and a spectral representation is necessary to describe adequately the flux of energy to smaller scales.

The BHR model also transports the net mass flux velocity, a, which appears in one of the mean flow pressure gradient source terms in the transport equations for $R_{i j}, K$, and $\varepsilon$. This transport equation for $\mathbf{a}$ is also derived from the Navier-Stokes equations in rigorous, but unclosed form, and then closed through modeling. To accomplish this derivation, the conservation of mass equation is multiplied by $\mathbf{u}^{\prime}$ and the conservation of momentum equation is multiplied by $\rho^{\prime}$. The flow variables of the two resulting equations are decomposed into mean and fluctuating parts, and the equations are then ensemble averaged (Besnard, et al.; 1992) to obtain

and

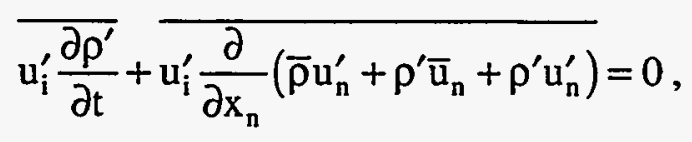

$$
\overline{\rho^{\prime} \frac{\partial u_{i}^{\prime}}{\partial t}}+\overline{\rho^{\prime} \bar{u}_{n} \frac{\partial u_{i}^{\prime}}{\partial x_{n}}}+\overline{\rho^{\prime} u_{n}^{\prime} \frac{\partial\left(\bar{u}_{i}+u_{i}^{\prime}\right)}{\partial x_{n}}}=-\overline{\left(\frac{\rho^{\prime}}{\bar{\rho}+\rho^{\prime}} \frac{\partial\left(\bar{p}+p^{\prime}\right)}{\partial x_{n}}\right)} .
$$


These two equation are added together to create a transport equation for $\overline{\rho^{\prime} \mathbf{u}^{\prime}}$, namely,

$$
\frac{\partial \overline{\rho^{\prime} u_{i}^{\prime}}}{\partial t}+\overline{u_{i}^{\prime} \frac{\partial\left(\bar{\rho} u_{n}^{\prime}+\rho^{\prime} \bar{u}_{n}+\rho^{\prime} u_{n}^{\prime}\right)}{\partial x_{n}}}+\overline{u_{n} \rho^{\prime} \frac{\partial u_{i}^{\prime}}{\partial x_{n}}}+\overline{\rho^{\prime} u_{n}^{\prime}} \frac{\partial \bar{u}_{i}}{\partial x_{n}}+\overline{\rho^{\prime} u_{n}^{\prime} \frac{\partial u_{i}^{\prime}}{\partial t}}=-\overline{\left(\frac{\rho^{\prime}}{\bar{\rho}+\rho^{\prime}}\right) \frac{\partial\left(\bar{p}+p^{\prime}\right)}{\partial x_{i}}} .
$$

The density-stress correlation can be rewritten as

$$
-\overline{\left(\left(\frac{\rho-\bar{\rho}}{\bar{\rho}+\rho^{\prime}}\right) \frac{\partial\left(\bar{p}+p^{\prime}\right)}{\partial x_{n}}\right)}
$$

and this can be separated and rewritten as

$$
\left[\bar{\rho} \overline{\left(\frac{1}{\rho}\right)}-1\right] \frac{\partial \bar{p}}{\partial x_{n}}+\bar{\rho} \overline{\left(\frac{1}{\rho}\right) \frac{\partial p^{\prime}}{\partial x_{n}}} .
$$

From the definition given in Chapter 2 ,

$$
b=-\rho^{\prime}\left(\frac{1}{\rho}\right)^{\prime},
$$

so that this correlation can be rewritten as

$$
b=\bar{\rho}\left(\frac{1}{\rho}\right)-1 .
$$

Because

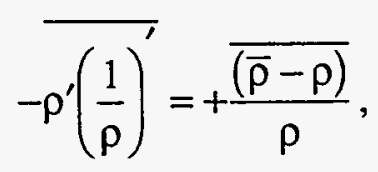

the resulting transport equation for a becomes

$$
\begin{aligned}
& \frac{\partial \bar{\rho} a_{i}}{\partial t}+\frac{\partial \bar{\rho} a_{i} \tilde{u}_{n}}{\partial x_{n}}+\bar{\rho} a_{n} \frac{\partial \bar{u}_{i}}{\partial x_{n}} \\
& =\frac{\overline{\rho^{\prime} u_{i}^{\prime} u_{n}^{\prime}}-R_{i n}}{\bar{\rho}} \frac{\partial \bar{\rho}}{\partial x_{n}}+\bar{\rho} \frac{\partial a_{i} a_{n}}{\partial x_{n}}-\frac{\partial \overline{\rho^{\prime} u_{i}^{\prime} u_{n}^{\prime}}}{\partial x_{n}}-b \frac{\partial \bar{p}}{\partial x_{n}}-\overline{\rho\left(\frac{1}{\rho}\right)^{\prime}\left(\frac{\partial p^{\prime}}{\partial x_{i}}\right)} .
\end{aligned}
$$

A gradient approximation is used to model the triple correlations, i.e.,

$$
\overline{\rho^{\prime} u_{i}^{\prime} u_{n}^{\prime}}=-C_{D a} \frac{K}{\varepsilon}\left(R_{i m} \frac{\partial a_{n}}{\partial x_{m}}+R_{m n} \frac{\partial a_{i}}{\partial x_{m}}\right)
$$


The modeling of the density-pressure correlation of the a equation is split into a part that responds to mean flow gradients, namely

$$
\mathrm{C}_{2 \mathrm{a}} \bar{\rho} \mathrm{a}_{\mathrm{n}} \frac{\partial \tilde{\mathrm{u}}_{\mathrm{i}}}{\partial \mathrm{x}_{\mathrm{n}}}
$$

and a part which represents a decay term of a, namely,

$$
-C_{1 a} \bar{\rho} \frac{\varepsilon}{K} a_{i}
$$

With the above modeling choices, the transport equation for $\mathbf{a}$ in the BHR model is

$$
\begin{aligned}
\frac{\partial \bar{\rho} a_{i}}{\partial t}+\frac{\partial \bar{\rho} a_{i} \tilde{u}_{n}}{\partial x_{n}} & +\bar{\rho} a_{n} \frac{\partial \bar{u}_{i}}{\partial x_{n}}=\bar{\rho} \frac{\partial a_{i} a_{n}}{\partial x_{n}}-C_{l a} \bar{\rho} \frac{\varepsilon}{K} a_{i}+C_{2 a} \bar{\rho} a_{n} \frac{\partial \tilde{u}_{i}}{\partial x_{n}} \\
& +b \frac{\partial \bar{p}}{\partial x_{i}}-\frac{R_{i n}}{\bar{\rho}} \frac{\partial \bar{\rho}}{\partial x_{n}}+C_{D_{a}} \bar{\rho} \frac{\partial}{\partial x_{m}}\left[\frac{K}{\varepsilon \bar{\rho}}\left(R_{i n} \frac{\partial a_{m}}{\partial x_{n}}+R_{m n} \frac{\partial a_{i}}{\partial x_{n}}\right)\right] .
\end{aligned}
$$

The quantity $b$, the specific volume-density correlation, is coupled to the principal driving term in the a equation. The BHR model also transports this quantity b. A transport equation for $\mathrm{b}$ is derived via the Reynolds decomposition of the conservation of mass equation coupled with the definition for specific volume, $v$ (see Chapter 2 ). The equation for the specific volume derived from the conservation of mass equation is

$$
\frac{\partial}{\partial t}\left(\frac{1}{v}\right)+\frac{\partial}{\partial x_{n}}\left(\frac{u_{n}}{v}\right)=0
$$

Substitution of the Reynolds decomposition coupled to ensemble averaging yields

$$
\frac{\partial \bar{v}}{\partial t}+\bar{u}_{n} \frac{\partial \bar{v}}{\partial x_{n}}=\bar{v} \frac{\partial \bar{u}_{n}}{\partial x_{n}}-\overline{u_{n}^{\prime} \frac{\partial v^{\prime}}{\partial x_{n}}}+\overline{v^{\prime} \frac{\partial u_{n}^{\prime}}{\partial x_{n}}} .
$$

Using Eq. (3.2.18) a transport equation for $\mathrm{b}$ can be derived from Eq. (3.2.26), namely,

$$
\frac{\partial b}{\partial t}+\bar{u}_{n} \frac{\partial b}{\partial x_{n}}+\left(\frac{b+1}{\bar{\rho}}\right) \frac{\partial \bar{\rho} a_{n}}{\partial x_{n}}+\bar{\rho} \frac{\partial}{\partial x_{n}} \overline{v^{\prime} u_{n}^{\prime}}=0 .
$$

For the case of the mixing layer studied in this work, Equation. (3.2.27) contains a correlation, $\overline{v^{\prime} u_{i}^{\prime}}$, that must be modeled. The BHR authors (Besnard, et al., 1987) have chosen to model this term as a diffusion term. We have improved the modeling of this term. 
Through careful geometrical considerations, an expression for the time evolution of singlepoint $b$ can be derived. In Appendix B we demonstrate, in two different ways, that this term should not be modeled as a diffusion term but rather as a modification to the source term. It could therefore be argued that there should not be a diffusion term in the b equation, because all the mass flux is already accounted for in the transport equation of $\mathbf{a}$. Thus, $\mathrm{b}$ should only react kinematically to the dynamical behavior of the rest of the equations of the model.

This concludes the abridged formal derivation and discussions of the single-point turbulence transport model equations for $\mathrm{R}_{\mathrm{ij}}, \mathrm{a}$, and $\mathrm{b}$. This brief exercise was included to provide a meaningful background on which to base some of the modeling ideas for the spectral model presented in Chapter 5, to contrast the single-point and two-phase flow models, and to provide motivation for a spectral turbulence transport model.

\subsection{Numerical results for the single-point turbulence transport approach}

Two computer codes were developed to study the capability of the BHR single-point turbulence transport model for describing the mix of two incompressible fluids subjected to a pressure gradient normal to their interface. They differ only in the coordinate system. One is Lagrangian, the coordinates moving with the net mass flux, $\bar{\rho} \mathbf{a}$. The other is Eulerian, with a fixed coordinate system. Each incorporates various consistency conditions in order to be compatible with the constraints of microscopic incompressibility. Results from the two independently-constructed codes were compared and found to have negligible differences. Results of calculations were also compared with those of a Lagrangian code for compressible flows, for examples at very low (but finite) Mach number, and again the agreement lends confidence to the validity of all three codes. The numerical result presented here is from calculations with the incompressible Eulerian code.

The calculations have concentrated on flows at vanishingly small Mach number. There are two fluids, with negligible molecular viscosity and diffusivity, so that the density of each element of either fluid remains constant (incompressible). The fluids are confined to 
a rigid-wall rectangular box that remains at rest, with an initially horizontal interface between them. A body force (acceleration) establishes a pressure gradient normal to the interface in the unstable direction, such that the higher pressure is in the lighter fluid. The fluids mix; with constant pressure drop the TMZ develops into a self-similar behavior in which the width, $W$, grows as

$$
\mathrm{W}=\mathrm{cons} \tan \mathrm{t} * \mathrm{At} * \mathrm{gt}^{2} \text {, }
$$

where the Atwood number, At, is defined as $\left(\rho_{1}-\rho_{2}\right) /\left(\rho_{1}+\rho_{2}\right), g$ is acceleration, and $t$ is time. Traditionally, the mix width is divided into two parts, namely, (1) $\mathrm{h}_{2}$ the spike penetration depth of the heavier fluid into the lighter fluid, and (2) $h_{1}$ the bubble penetration depth of the lighter fluid into the heavier fluid,. A self-similar coefficient, $\alpha$, is defined such that

$$
\mathrm{h}_{1}=\alpha(\mathrm{At}) \mathrm{gt}^{2}
$$

Most experimental investigators (Read \& Youngs, 1983; Read, 1984; Smeeton \& Youngs, 1987; Andrews, 1992; Linden et al., 1992; Youngs, 1992a) agree that for non-diffusive fluids, $\alpha=0.065 \pm 0.015$. The configuration is statistically one-dimensional in physical space, and the effects of the box walls are considered negligible. (Experimental techniques to ensure this consideration are not trivial). Our calculations develop the self similarity by advancing through time from a prescribed initial state.

For the turbulent fluid mixing calculations there are three principal criteria for merit.

(1) The calculation must be capable of evolving from arbitrary initial conditions to self-similarity that does not depend on initial conditions.

(2) The depth of the bubble envelop penetration into the heavier fluid, $h_{1}$, must grow at the proper rate as determined experimentally by Read (1984) and Youngs (1989), as

$$
\mathrm{h}_{1}=\alpha \mathrm{X} \text {, }
$$

where $\mathrm{X}=(\mathrm{At}) * \mathrm{~g} * \mathrm{t}^{2}$, with $\alpha$ close to 0.065 , together with the correct experimentally observed ratio between $h_{1}$ and $h_{2}$ which depends on Atwood number. 
(3) The profiles of $\bar{\rho}$ (and thus of $\alpha_{1}, \alpha_{2}$, and of $c_{1}$ and $c_{2}$ ) through the TMZ must exhibit the relatively smooth (monotonic) character of the experiments of Youngs (1989) and Read (1984) and Kucherenko, et al. (1991).

This third requirement has been the most challenging to achieve with the BHR singlepoint turbulence transport model.

There are, of course, other criteria for merit, but currently available experimental results do not permit their testing. These include, most especially, the ratio of turbulence energy to $\mathrm{U}^{2}$, which should vary only with Atwood number in a "universal" fashion. Smoothness of profiles for $\mathbf{a}$ and $\mathrm{b}$ are also useful for discriminating merit. However, we anticipate experiments that are capable of measuring these additional quantities to begin in the near future at Lawrence Livermore National Laboratory under the direction of Guy DeMonte and Bruce Remington (Remington, personal comm., 1995).

For incompressible flow in a box at rest, there is no net volume flux, and $\overline{\mathbf{u}}$ (in the $\mathrm{y}$ direction, also) equals zero, so that $\tilde{\mathbf{u}}=\mathbf{a}$. It is through the incompressibility condition and this equality of $\tilde{\mathbf{u}}$ and $\mathbf{a}$ that the pressure profile across the mixing layer is determined.

The differential equations solved by the incompressible Eulerian code are the following:

$$
\begin{gathered}
\frac{\partial \bar{\rho}}{\partial t}+\frac{\partial \bar{\rho} \tilde{u}}{\partial y}=0 \\
\frac{\partial \bar{\rho} \tilde{u}}{\partial t}+\frac{\partial \bar{\rho} \tilde{u}^{2}}{\partial y}=-\frac{\partial}{\partial y}\left(\bar{p}+R_{y y}\right)+\bar{\rho} g \\
\frac{\partial \bar{\rho} K}{\partial t}+\frac{\partial \bar{\rho} K \tilde{u}}{\partial y}=a \frac{\partial \bar{p}}{\partial y}-R_{y y} \frac{\partial \tilde{u}}{\partial y}+\frac{\partial}{\partial y}\left(\bar{\rho} v_{t} \frac{\partial K}{\partial y}\right)-\bar{\rho} \varepsilon \\
\frac{\partial \bar{\rho} a}{\partial t}+\frac{\partial \bar{\rho} a \tilde{u}}{\partial y}=b \frac{\partial p}{\partial y}-\frac{R_{y y}}{\bar{\rho}} \frac{\partial \bar{\rho}}{\partial y}-C_{a 1} \frac{\bar{\rho} a \sqrt{K}}{S} \\
b=\frac{\alpha_{1} \alpha_{2}\left(\rho_{1}-\rho_{2}\right)^{2}}{\rho_{1} \rho_{2}},
\end{gathered}
$$




$$
\begin{gathered}
\frac{\partial \bar{\rho} S}{\partial t}+\frac{\partial \bar{\rho} S \tilde{u}}{\partial y}=\frac{\partial}{\partial y}\left(\frac{\bar{\rho} v_{t}}{\sigma_{\varepsilon}} \frac{\partial S}{\partial y}\right)-C_{s 3} \bar{\rho} S \frac{\partial \tilde{u}}{\partial y} \\
\frac{S}{K}\left[\left(\frac{3}{2}-C_{s 4}\right) \frac{\partial \bar{p}}{\partial y}-\left(\frac{3}{2}-C_{s 1}\right) R_{y y} \frac{\partial \tilde{u}}{\partial y}\right]-\left(\frac{3}{2}-C_{s 2}\right) \bar{\rho} \sqrt{K}, \\
\tilde{u}=a, \\
\varepsilon=\frac{K \sqrt{K}}{S}, \\
R_{y y}=\frac{2}{3}\left(\bar{\rho} K-2 \bar{\rho} v_{t} \frac{\partial \tilde{u}}{\partial y}\right), \\
\bar{\rho}=\alpha_{1} \rho_{1}+\alpha_{2} \rho_{2}, \\
\alpha_{1}+\alpha_{2}=1,
\end{gathered}
$$

and

$$
v_{\mathrm{t}}=(0.09) \mathrm{S} \sqrt{\mathrm{K}} .
$$

The BHR authors (BHR, et al. 1987) have elected to use the configurational form for $\mathrm{b}(\mathrm{y}, \mathrm{t})$ as described in Chapter 2; Chapter 5 along with Appendix B describes a much more general transport description for this variable in spectral space. The transport equation for the turbulent kinetic energy dissipation rate, $\varepsilon$, is replaced by a transport equation for the length scale, $S$, of the turbulent kinetic energy, K. Previous experience (Daly, personal comm., 1994) with using these equations for mixing problems has shown that use of an $\varepsilon$ transport equation can lead to deleterious behavior near discontinuities in the flow, i.e., shocks (Daly, 1992). The authors of BHR also found that it is easier to implement boundary and initial conditions for the length scale $S$ as opposed to $\varepsilon$. These two variables are related by Eq. (3.3.11).

The six phenomenological constants are assigned the following values (Daly, 1992):

$$
\sigma_{\varepsilon}=1.3, C_{\mathrm{a} 1}=2.25, \mathrm{C}_{\mathrm{s} 1}=1.55, \mathrm{C}_{\mathrm{s} 2}=2.0, \mathrm{C}_{\mathrm{s} 3}=0.01, \mathrm{C}_{\mathrm{s} 4}=1.35
$$


In the cell that contains the initial fluid interface, the length scale is initialized as $S_{0}=1 \times 10^{-5}$, and $b$ is assigned its maximum configurational value when $\alpha_{1}=\alpha_{2}=0.5$. The numerical results were found to be insensitive to variations (within reason) in the initial values of these two variables For the numerical results of the above turbulence transport equations applied to a mixing circumstance, refer to Fig. 3.3.1. This result is for the incompressible case of a heavy fluid, $\rho=2.0$, sitting over a lighter fluid, $\rho=1.0$, with an acceleration of $g=-1.0$ applied to the system in such a way as to initiate mixing, i.e., the extended RayleighTaylor instability. The configuration just described corresponds to the lighter fluid occupying the region of $y<0$ and the heavier fluid occupying the region of $y>0$. For the mixing case, the acceleration vector is pointing from top to bottom, which establishes a pressure gradient in the fluid that is consistent with the highest pressure residing in the lighter fluid. The calculation is carried out to a time of 30 to show some of the difficulty encountered when applying this version of the BHR model to this mixing problem.

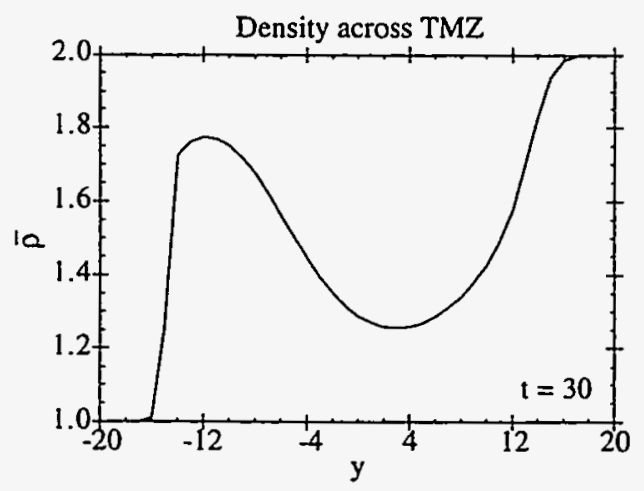

Figure 3.3.1. Density profile across $\mathrm{TMZ}$ at $\mathrm{t}=30$.

This "cleave" of fluid that is observed in the TMZ in Fig. 3.3.1 has led the BHR authors to consider a different formulation for the transport of mass. As background investigations for this report, some variations in the modeling of the transport of the mass-flux variable, a, were tried, but to no avail. The experience of code implementation and testing has led the BHR authors to introduce a conceptual inconsistency into the model formulation. The reason is purely empirical. To formulate a model that is at least monotonic through the TMZ, the following strategy was implemented by the authors. 
Due to incompressibility, the mass concentrations of a two fluid problem, $c_{1}$ and $c_{2}$, can be determined uniquely from $\bar{\rho}$, and the mass conservation equation can be transformed into the following transport equations for $c_{1}$ and $c_{2}$ :

and,

$$
\frac{\partial \bar{\rho} c_{1}}{\partial t}+\frac{\partial \bar{\rho} c_{1} \tilde{v}}{\partial y}=-\frac{\partial}{\partial y}\left(\frac{\rho_{1} \rho_{2} \mathrm{a}}{\rho_{2}-\rho_{1}}\right)
$$

$$
\frac{\partial \bar{\rho} c_{2}}{\partial t}+\frac{\partial \bar{\rho} c_{2} \tilde{v}}{\partial y}=-\frac{\partial}{\partial y}\left(\frac{\rho_{1} \rho_{2} \mathrm{a}}{\rho_{1}-\rho_{2}}\right) \text {. }
$$

Figure 3.3.1 shows that the fluxes of mass fractions, $c_{1}$ and $c_{2}$, given by

$$
\frac{\rho_{1} \rho_{2} \mathrm{a}}{\rho_{1}-\rho_{2}} \quad \text { and } \quad \frac{\rho_{1} \rho_{2} \mathrm{a}}{\rho_{2}-\rho_{1}}
$$

respectively, do not agree well with the results from experiments.

An empirically effective variation was suggested by R. Rauenzahn (personal comm., 1994). In the mass-fraction transport equations he replaces the species flux by a diffusive form, namely,

and,

$$
\frac{\rho_{1} \rho_{2} \mathrm{a}}{\rho_{1}-\rho_{2}} \rightarrow \frac{\bar{\rho} v_{t}}{\sigma_{c}} \frac{\partial c_{1}}{\partial y}
$$

$$
\frac{\rho_{1} \rho_{2} \mathrm{a}}{\rho_{2}-\rho_{1}} \rightarrow \frac{\bar{\rho} v_{1}}{\sigma_{c}} \frac{\partial c_{2}}{\partial y}
$$

The justification of this gradient flux form for a can be "derived" from the a equation by neglecting all terms except the decay term and the density gradient "source" term in Eq. (3.3.7), which is the so-called homogeneous, steady state balance approximation. This approximation assumes that once a steady-state condition is reached, a balance is set up by the source of turbulent energy and the decay, i.e.,

$$
0=-\frac{R_{y y}}{\bar{\rho}} \frac{\partial \bar{\rho}}{\partial y}-C_{a 1} \frac{\bar{\rho} a \sqrt{K}}{S},
$$

and since, from the Boussinesq approximation,

$$
R_{y y} \approx(\text { Constant } t) K,
$$


the expression for the net mass flux relative to $\overline{\mathbf{u}}$ can be expressed as

where,

$$
\bar{\rho} \mathbf{a} \approx \frac{v_{t}}{\bar{\rho}} \frac{\partial \bar{\rho}}{\partial y},
$$

$$
v_{t}=(0.09) S \sqrt{K}
$$

The inconsistency of this modeling choice lies in the use of a transported $\mathbf{a}$ in the equations for $\bar{\rho}, K$, and $S$, but a diffusive representation of a for the species mass-fraction transport. Although $c_{1}$ and $c_{2}$ should be uniquely determined as functions of $\bar{\rho}$, this treatment violates that constraint. The virtue of his approach, however, lies in the relatively good results that are obtained for the comparisons with experiments.

The equations expressed in this inconsistent form describe a particular form of the more general BHR equations, which has seen extensive implementation and testing in large multi-dimensional fluid dynamics codes (F.H. Harlow, personal comm.) at Los Alamos National Laboratory.

The substitution of the diffusive form into the species transport equation eliminates the cleave that is seen in Fig. 3.3.1; however, the divorce of the transported a from the transport of the species prohibits any counter-gradient fluxing of species. That is to say, the model cannot accurately predict any type of demixing due to a reversal of acceleration (rapid transient). For that matter, it is incapable of representing any type of transient in the driver that would produce a counter-gradient flux of mass.

Another motivation for this part of the study was to examine different closures to replace the diffusive type closures used to model the triple correlations of the single-point turbulence transport equations. We tried to use the configurational techniques described in Chapter 2 to derive a modeling of the triple correlation found in the a equation, $\overline{\rho^{\prime} u_{i}^{\prime} u_{j}^{\prime}}$, as

$$
\overline{\rho^{\prime} u_{i}^{\prime} u_{j}^{\prime}}=\bar{\rho} a_{i} a_{j}\left(1-\frac{1}{B}+\frac{1}{b}\right)
$$

This procedure gives a wave-like flux term in the a equation, which seems appropriate when we have situations of inadequate mass fluxing in strongly driven mixing type flows, which 
we have observed in some of our numerical tests. Major difficulties occurred with the $b$ and B that appear in the denominator, which quickly deterred any further investigations.

\subsection{Summary of the single-point turbulence transport approach}

As a comment on this approach, it should be noted that the construction of singlepoint turbulence transport equations has been saturated with the introduction of heuristic modeling terms for nearly three decades. Our goal in this Chapter is not to demonstrate conceptual or empirical validity for this derivational procedure. We seek instead to indicate the effects of a representative set of modeling terms as background for the spectral techniques that we propose in Chapter 5.

We believe, however, that the difficulties with current single-point transport equations for mixing-layer calculations are much more fundamental, and that alleviation of the problems cannot be accomplished satisfactorily with simple variants of the current formulation. As shown in Chapters 5 and 6, there are numerous clues to transport-variable structure as revealed in a spectral formulation of the equations.

While all of the single-point turbulence transport theories may give respectable results for developed turbulent flows, they are severely limited in the case of rapid transients, like in generalized Rayleigh-Taylor mixing layers or other transitional flows that are of interest to the engineer. In these cases, i.e., when the time scales for the turbulence and the mean flow are the same, one cannot assume spectral equilibrium. Hence, the fundamental principles upon which the single-point transport theories are constructed are no longer valid. In this light, the motivation for the development of a spectral model for generalized turbulent flow is clear.

Motivation for the formulation of a spectral model can be found when examining all of the shortcomings of the single-point model of this section. One implicit problem of the single-point equations that has been alluded to in this section is the absence of any type of length scale which can have a meaningful relationship to the turbulent flow. This shortcom- 
ing is manifested in the derivation of the Reynolds stress transport equation. To understand the scales associated with turbulence, we use the fact that the Navier-Stokes equations can be written at two different points in space. It is this fact that allows us to assumes that the twopoint correlations contain all of the necessary information about the correlations of fluctuations. These correlations are formulated in terms of the principal variable, $r$, which is the separation vector. Our approach has been to Fourier transform relative to the separation variable $\mathbf{r}$ in order to obtain a spectral description from which we can examine the Reynolds stress tensor for all wave numbers. Each procedure, i.e., whether we work with $\mathbf{r}$ or $\mathbf{k}$ as the primary variable, has its advantages and disadvantages, we choose $\mathbf{k}$. The weaknesses of the single-point theory that the spectral approach can address are the following:

(1) The single-point $R_{\mathrm{ij}}$ equation contains the energy decay term $\varepsilon_{\mathrm{ij}}$, where (Daly and Harlow; 1970)

$$
\varepsilon_{\mathrm{ij}}=2 v_{\mathrm{m}} \overline{\frac{\partial \mathrm{u}_{\mathrm{i}}^{\prime}}{\partial \mathrm{x}_{\ell}} \frac{\partial \mathrm{u}_{\mathrm{j}}^{\prime}}{\partial \mathrm{x}_{\ell}}}
$$

By definition, this term is directly associated with the decay of the turbulent kinetic energy due only to the molecular viscosity, and this requires the understanding of local fluctuating velocity gradients. The other term associated with the viscous decay includes the viscosity multiplied by the second derivative of $\mathrm{R}_{\mathrm{ij}}$. Daly and Harlow (1970) show by order of magnitude arguments that this latter term can be neglected when compared to the term of Eq. (3.4.1). To understand how these velocities in Eq. (3.4.1) change over small regions of space requires knowledge of a flow length scales and how the turbulence behaves at all scales. This is an impossibility with a single-point model. As shown by the authors of $\mathrm{BHRZ}, \varepsilon_{\mathrm{ij}}$ is more appropriately associated with (in general) the rate of energy transfer from the large (important scales) to the fine scales structures that have relatively little effect on the mean behavior of the flow. It is only in a circumstance of spectral equilibrium, that the two different interpretations of $\varepsilon_{\mathrm{ij}}$ are equivalent.

(2) The identification of the various dominate scales of the flow, i.e., a separate length scale for each of the following, $a_{i}, b, R_{n n}$, and any other components of the Reynolds 
stress tensor that are relevant to the particular problem at hand. With spectral self-similarity these scales are all proportional to one another. In some important drive circumstances of interest, however, this proportionality is lost, and the spectral issues must be addressed to see how the ratios of the dominant length scales change. With the full spectral equations, however, the rapid transient of the driving source terms that is responsible for destroying the spectral self-similarity can nevertheless be completely represented.

(3) An implicit but essential constraint for the validity of single-point turbulence transport equations is the existence of constraints that are powerful enough to ensure spectral self-similarity. Otherwise the infinite number of degrees of freedom cannot be characterized by the small number of variables that occur in the single-point formulation. (This is in close analogy to the example of molecular dynamics in which the universal self-similar form of the Maxwell-Boltzmann distributions allow for meaningful identification of such variables as a pressure, temperature, density, and velocity). Spectral transport equations enable the determination of self-similar forms, and the determination of rates of return to these self-similar forms once a transient in the driving source term occurs (Clark, 1992). Spectral formulations also enable us to determine strongly anisotropic self-similar forms in circumstances of inhomogeneity. Hence, they can be used as a basis to generalize current single-point models and characterize the circumstances in which the single-point models are valid. An example of this is to be able to characterize the competition between a transient driver and other equilibrating terms which dictate a rate of return to self-similarity. 


\section{Two-Field Flow Modeling}

\subsection{Introduction}

In view of what is presented in Chapter 2 and Appendix $\mathrm{C}$ concerning the ordered components of a mixing problem, a set of the two-field flow equations is presented. In this chapter different strategies to overcome some of the difficulties associated with the two-field flow equations are discussed. For comparison with the turbulence-transport model presented in Chapter 3 we have included calculations of the low-speed mix problem using two-field flow equations. More specifically, we have made comparisons with the experiments of Youngs (1992a) and Burrows, Smeeton and Youngs (1984) that examine the turbulent mixing due to the late self-similar stages of Rayleigh-Taylor instability.

This chapter is included to outline some of the potential modeling strategies that one can use from the two-field theory to supplement the single-field theory of Chapter 3 , and to review some of the techniques that serve as a basis for the development of the spectral transport model of Chapter 5. We also use this opportunity to discuss some of the modeling assumptions that go into a two-field model such as this one. These discussions serve as a motivation for developing a spectral turbulence transport model for describing variable density turbulent mixing.

\subsection{Two-field approach}

Two-field modeling for mix problems works with the configuration variables described in Chapter 2, i.e., $\alpha_{1}, \alpha_{2}, \rho_{1}, \rho_{2}, u_{1}, u_{2}$, and follows the evolution of these variables through time. There are two levels of modeling that have been incorporated into the twofield formulation (Youngs, 1992b). The first of these is the "up-front" modeling that relates the momentum sharing between fluids to the drag that would be felt by an individual parcel of one fluid moving through the other. The second is the extended modeling that (1) modifies the drag coefficients to represent effects of interface contortion, (We confirm 
Youngs (1992b) requirement of an order-of-magnitude greater drag coefficient than that of an individual sphere. This is expected because the drag term for a sphere contains a fixed diameter in the denominator while our drag term contains the width of the evolving TMZ in the denominator.) (2) that modifies the scale of drag (in proportion to the relative velocity of interpenetration between the two fields; see Eq. (4.2.9)) to account for bubble doubling or clumping of particles, in recognition that the principal drag is that of the clumps, not the particles, and (3) that replaces the molecular viscosity in the viscous drag term by a simple representation of the turbulent viscosity, $v_{t}$, where, for example, we could put

$$
v_{\mathrm{t}}=0.09 \mathrm{~S} \sqrt{\mathrm{K}} \text {. }
$$

$\mathrm{S}$ is the clump (turbulence) scale, and $\mathrm{K}$ is determined from either of two models. The first of these assumes that the disordered part of the turbulence energy can be approximated as a prescribed fraction, $\mathrm{K}_{0}$, of the ordered part of Eq. (2.2.39), namely,

$$
\mathrm{K}=\mathrm{K}_{0} \frac{\alpha_{1} \alpha_{2} \rho_{1} \rho_{2}}{2 \bar{\rho}^{2}}\left(\mathrm{u}_{1}-\mathrm{u}_{2}\right)^{2},
$$

or, the second (Youngs, 1992b \& 1989) transforms ordered-energy loss from drag completely to disordered energy as an intermediate step on the way to heat, i.e.,

$$
\frac{\mathrm{dK}}{\mathrm{dt}}=\text { (source from energy loss due to drag). }
$$

This addition of a disordered component to the flow by which to achieve a further degree of interpenetration beyond that of the ordered velocities of the materials was dictated by the excessive rate of demixing in calculations when the body force was reversed. For our needs in this chapter, we have not, however, incorporated this diffusive effect into the calculations.

Youngs (1992b) and Cranfill (1991) assume that the drag scale changes at a rate proportional to the relative velocity of interpenetration; note that self-similarity for RayleighTaylor linear stability analysis is achieved by this assumption, as described in Section 6.2.

Two-field flow equations have an advantage over the single-field turbulence transport equations when describing mix problems by keeping two separate fields to allow for the 
tracking of mixtures fractions at any point. The two-field continuum flow equations can take on many forms, depending on the amount of flow detail the researcher wishes to capture, i.e., phase transitions between fluids, virtual mass effects, etc. The set of differential equations governing two-field flow for the case of constant microscopic densities, which we present here, is

$$
\begin{gathered}
\frac{\partial \rho_{1} \alpha_{1} u_{1 i}}{\partial t}+\frac{\partial p_{1} \alpha_{1} u_{1 i} u_{1 n}}{\partial x_{n}}=-\alpha_{1} \frac{\partial p}{\partial x_{i}}+K_{D} \bar{\rho}\left(u_{2 i}-u_{1 i}\right)+\rho_{1} \alpha_{1} g_{i} \\
\frac{\partial \rho_{2} \alpha_{2} u_{2 i}}{\partial t}+\frac{\partial \rho_{2} \alpha_{2} u_{2 i} u_{2 n}}{\partial x_{n}}=-\alpha_{2} \frac{\partial p}{\partial x_{i}}+K_{D} \bar{\rho}\left(u_{1 i}-u_{2 i}\right)+\rho_{2} \alpha_{2} g_{i} \\
\frac{\partial \alpha_{1}}{\partial t}+\frac{\partial \alpha_{1} u_{1 n}}{\partial x_{n}}=0, \\
\frac{\partial \alpha_{2}}{\partial t}+\frac{\partial \alpha_{2} u_{2 n}}{\partial x_{n}}=0, \\
K_{D}=\left(\frac{3}{8}\right) \frac{\alpha_{1} \alpha_{2}}{S}\left[C_{D}\left|u_{2}-u_{1}\right|+\frac{12 v_{t}}{S}\right], \\
\frac{d S}{d t}=\beta\left|u_{2}-u_{1}\right|,
\end{gathered}
$$

and,

$$
v_{\mathrm{t}}=0.09 \mathrm{~S} \sqrt{\mathrm{K}}
$$

Here $\mathrm{S}$, the clump scale has replaced the particle radius in the terms for form and viscous drag. The turbulent kinetic energy, $K$, can be found by either of the two ways shown by Eq. (4.2.2) or (4.2.3). The coefficient $\beta$ is the fractional value of the TMZ width that the dominant scales of the flow appear to be as observed in experiments (Youngs, 1992b).

The momentum transfer term, Eq. (4.2.8), is made up of a form drag component as well as a viscous drag component. Upon substitution of Eq. (4.2.2) and (4.2.10) into the turbulent viscosity, it can be seen that both the form drag and the viscous drag are proportional to the relative velocity between the two fields. Thus, for this study, we have neglected the viscous part of the exchange term and work with only the form drag. As a consequence of 
this, we never introduce a disordered component into the flow. The set of differential equations that we solve numerically are given in the next section.

In addition to solving equations for the primary two-field variables, we can use these equations to derive other equations that describe the variations of configurational versions of $a_{j}, b$, and $R_{i j}$, and compare these with the single-field transport equations of Section 4 of this Chapter. The goal is to provide guidance for closure modeling for the single-point turbulence transport equations. A transport equation for $\mathbf{a}$ is derived in terms of two-field variables by making use of the following relation between the net mass flux velocity and the mean flow velocity,

$$
\bar{\rho} \mathbf{a}=\bar{\rho} \tilde{\mathbf{u}}-\bar{\rho} \overline{\mathbf{u}} .
$$

Recognizing that these two-field equations without augmentation represent only the ordered component of the interpenetrating process allows us to take advantage of their seemingly well behaved nature within the structure of the mixing layer. Perhaps guidance to the modeling of the single-point turbulence transport equations can be taken from the transport equation for a derived from two-field variables.

Thus, we need transport equations for $\tilde{\mathbf{u}}$ and $\overline{\mathbf{u}}$. For the transport equation of $\tilde{\mathbf{u}}$ we add the two momentum equations, Eq. (4.2.4) and (4.2.5), and make use of the expression for the total momentum per unit volume,

$$
\bar{\rho} \tilde{u}_{i}=\alpha_{1} \rho_{1} u_{1 i}+\alpha_{2} \rho_{2} u_{2 i},
$$

and the expression for average density,

$$
\bar{\rho}=\alpha_{1} \rho_{1}+\alpha_{2} \rho_{2},
$$

to obtain the following transport equation for the total momentum density:

$$
\frac{\partial \bar{\rho} \tilde{u}_{i}}{\partial t}+\frac{\partial\left(\alpha_{1} \rho_{1} u_{1 i} u_{1 n}+\alpha_{2} \rho_{2} u_{2 i} u_{2 n}\right)}{\partial x_{n}}=-\frac{\partial p}{\partial x_{i}}+\bar{\rho} g_{i}
$$

From Chapter 2, the sum in the advective derivative can be expressed as

$$
\left(\alpha_{1} \rho_{1} u_{1 i} u_{1 n}+\alpha_{2} \rho_{2} u_{2 i} u_{2 n}\right)=\tilde{u}_{i} \tilde{u}_{n} \bar{\rho}+\frac{\bar{\rho} a_{i} a_{n}}{b}
$$


Thus, the transport equation for the total momentum per unit volume can be expressed as

$$
\frac{\partial \bar{\rho} \tilde{u}_{i}}{\partial t}+\frac{\partial \bar{\rho} \tilde{u}_{i} \tilde{u}_{n}}{\partial x_{n}}=-\frac{\partial}{\partial x_{n}}\left(\frac{\bar{\rho} a_{i} a_{n}}{b}\right)-\frac{\partial p}{\partial x_{i}}+\bar{\rho} g_{i}
$$

One thing that should be noted here is the identification of the Reynolds stress tensor that emerges from the two-field flow description of the mean flow equation, namely,

$$
R_{i j}=\frac{\vec{\rho} a_{i} a_{j}}{b} .
$$

This form for $\mathrm{R}_{\mathrm{ij}}$ can be thought of as the ordered component of the Reynolds stress tensor since it emerges from these equations.

A transport equation for the mean flow velocity, $\overline{\mathbf{u}}$, is derived in the same way. We add the two momentum transport equations, which for constant microscopic densities, can be written as

and,

$$
\frac{\partial \alpha_{1} u_{1 i}}{\partial t}+\frac{\partial \alpha_{1} u_{1 i} u_{1 n}}{\partial x_{n}}=-\frac{\alpha_{1}}{\rho_{1}} \frac{\partial p}{\partial x_{i}}+\frac{K_{D} \bar{\rho}\left(u_{2 i}-u_{1 i}\right)}{\rho_{1}}+\alpha_{1} g_{i}
$$

$$
\frac{\partial \alpha_{2} \mathrm{u}_{2 \mathrm{i}}}{\partial \mathrm{t}}+\frac{\partial \alpha_{2} \mathrm{u}_{2 \mathrm{i}} \mathrm{u}_{2 \mathrm{n}}}{\partial \mathrm{x}_{\mathrm{n}}}=-\frac{\alpha_{2}}{\rho_{2}} \frac{\partial \mathrm{p}}{\partial \mathrm{x}_{\mathrm{i}}}+\frac{\mathrm{K}_{\mathrm{D}} \bar{\rho}\left(\mathrm{u}_{1 \mathrm{i}}-\mathrm{u}_{2 \mathrm{i}}\right)}{\rho_{2}}+\alpha_{2} \mathrm{~g}_{\mathrm{i}}
$$

Referring to Chapter 2, the terms in the advective derivative can be expressed as

$$
\frac{\partial}{\partial x_{n}}\left(\alpha_{1} u_{l i} u_{l n}+\alpha_{2} u_{2 i} u_{2 n}\right)=\frac{\partial}{\partial x_{n}}\left(\bar{u}_{i} \bar{u}_{n}+\frac{a_{i} a_{n}}{B}\right),
$$

and, after multiplication by $\bar{\rho}$, coupled with the incompressibility condition for the mean flow, the resulting transport equation for $\bar{\rho} \overline{\mathbf{u}}$ is

$$
\frac{\partial \bar{\rho} \bar{u}_{i}}{\partial t}+\frac{\partial \bar{\rho} \bar{u}_{i} \bar{u}_{n}}{\partial x_{n}}=-\bar{u}_{i} \frac{\partial \bar{\rho} a_{n}}{\partial x_{n}}-\bar{\rho} \frac{\partial}{\partial x_{n}}\left(\frac{a_{i} a_{n}}{B}\right)-\bar{\rho}\left(\frac{\alpha_{1}}{\rho_{1}}+\frac{\alpha_{2}}{\rho_{2}}\right) \frac{\partial p}{\partial x_{i}}+K_{D} \bar{\rho}^{2}\left(u_{2 i}-u_{1 i}\right)\left(\frac{1}{\rho_{1}}-\frac{1}{\rho_{2}}\right) .
$$

The transport equations for $\tilde{\mathbf{u}}$ and $\overline{\mathbf{u}}$ are then combined to form the following equation for $\mathbf{a}$ :

where,

$$
\begin{aligned}
\frac{\partial \bar{\rho} a_{i}}{\partial t}+\frac{\partial \bar{\rho} a_{i} \tilde{u}_{n}}{\partial x_{n}}= & -\frac{\partial}{\partial x_{n}}\left[\bar{\rho} a_{i} a_{n}\left(\frac{1}{b}-\frac{1}{B}\right)\right]-\frac{\partial \bar{\rho} a_{i} \bar{u}_{n}}{\partial x_{n}}-\frac{a_{i} a_{n}}{B} \frac{\partial \bar{\rho}}{\partial x_{n}} \\
& -\bar{\rho} a_{n} \frac{\partial \bar{u}_{i}}{\partial x_{n}}+b \frac{\partial p}{\partial x_{n}}-K_{D}^{*} \bar{\rho} a_{i}
\end{aligned}
$$

$$
K_{D}^{*}=\frac{K_{D} \bar{\rho}^{2}}{\alpha_{1} \alpha_{2} \rho_{1} \rho_{2}} \text {. }
$$


Equation (4.2.20) can be used to suggest new modeling ideas for the a equation in the singlepoint turbulence transport model.

In this section, the two-field flow equations are presented so as to get an idea of the types of terms that represent the ordered part of a mixing flow. In Chapter 3, we presented the single-point turbulence transport equations and pointed out diffusive modeling features that could be aided by the equations of this section. As part of the background investigations for this study, we attempted to enhance the diffusive closure modeling for some of the singlepoint turbulence transport terms with ideas derived from the two-field equations of this section (R. Rauenzahn personal communication, 1994). In following the spirit of LA-12303-MS (BHR), we attempted to model some of the spatial transport terms of the BHR model using their two-phase equivalence. Some features of the numerics (singularities) prohibited us from propagating the turbulence into a stationary fluid (as is the case for the spreading TMZ of the Rayleigh-Taylor instability). We quickly abandoned this approach and restricted our work to using the two-fluid model to demonstrate the ideas as discussed in the summarizing section of this chapter. However, the work of Cranfill (1991 \& 1992) demonstrates the essence of this type of a hybrid type model for turbulent mixing. The primary function of these types of models is to capture both the "ordered" and the "disordered" parts of an interpenetrating flow.

\subsection{Numerical results for the two-field approach}

In this section we show numerical results from a set of two-field equations. The set of equations that are numerically solved here are those discussed by Harlow and Amsden (1975) with one modification; an evolving length scale is used in the momentum exchange term instead of a fixed length scale representing particle size. Such things as phase transition and virtual mass terms have all been neglected. The resulting set of one-dimensional equations for which numerical solutions are given is 


$$
\begin{gathered}
\frac{\partial \rho_{1} \alpha_{1} u_{1}}{\partial t}+\frac{\partial \rho_{1} \alpha_{1} u_{1} u_{1}}{\partial y}=-\alpha_{1} \frac{\partial p}{\partial y}+K_{D} \bar{\rho}\left(u_{2}-u_{1}\right)+\rho_{1} \alpha_{1} g \\
\frac{\partial \rho_{2} \alpha_{2} u_{2}}{\partial t}+\frac{\partial \rho_{2} \alpha_{2} u_{2} u_{2}}{\partial y}=-\alpha_{2} \frac{\partial p}{\partial y}+K_{D} \bar{\rho}\left(u_{1}-u_{2}\right)+\rho_{2} \alpha_{2} g \\
\frac{\partial \alpha_{1}}{\partial t}+\frac{\partial \alpha_{1} u_{1}}{\partial y}=\sigma \frac{\partial^{2} \alpha_{1}}{\partial y^{2}} \\
\frac{\partial \alpha_{2}}{\partial t}+\frac{\partial \alpha_{2} u_{2}}{\partial y}=\sigma \frac{\partial^{2} \alpha_{2}}{\partial y^{2}} \\
K_{D}=C_{D}\left(\frac{3}{8}\right) \frac{\bar{\rho} \alpha_{1} \alpha_{2}\left|u_{2}-u_{1}\right|}{S} \\
S=\beta W
\end{gathered}
$$

and,

$$
\bar{\rho}=\alpha_{1} \rho_{1}+\alpha_{2} \rho_{2} .
$$

Where $W$ is the width of the TMZ, and $\alpha_{1}+\alpha_{2}=1$. Equations (4.3.3) and (4.3.4) are derived from the conservation of mass equation using Eq. (4.3.7) for the definition of the average density. The $\mathrm{K}_{\mathrm{D}}$ term in the momentum equation represents the momentum exchange between fields and is derived on the basis of a single particle falling through a liquid. Applying these equations to the case of two interpenetrating fluids, we have found through investigation that the drag coefficient, $\mathrm{C}_{\mathrm{D}}$, should, for reasonable results, be increased at least an order of magnitude compared to the nominal values of about 1.0 for a single sphere falling through a liquid. Hence, for these calculations, we have set $C_{D}=12.0$, which is consistent with the value that Youngs (1992b) needed in order to match experimental mixing rates (Smeeton \& Youngs, 1987). We speculate that the need for an enhanced drag coefficient arises due to the fact that the complicated configuration of the TMZ for two interpenetrating fluids produces much enhancement to the inter fluid surface drag as a result of dead-end channels and the blunt mushroom-like tips at the end of penetrating spikes of fluid. Another way to explain this is that the "effective sphere" size becomes more closely approximated by $S / 10$ or $S / 20$ due to our replacement of the TMZ scale in the denominator of the momentum exchange term. 
Most cases of two interpenetrating fields are inherently unstable. It has been shown through the use of linear stability analysis (Murray, 1965; Travis, Harlow \& Amsden, 1976) that the above set of equations is unconditionally unstable. It is well known that many simple multi-field models give rise to an ill-posed equation set (Jones \& Prosperetti, 1985). These instabilities represent the physical instabilities known to be present in interpenetrating flows. As was stated earlier in this chapter, the two-field equations are properly applicable to only the ordered part of the flow and small fluctuations away from a purely ordered flow. It is this differential instability that represents the tendency for transition to the disordered components of the flow, namely the turbulence at all scales and in all three directions. These physical instabilities add diffusive transport to the species equations, which enhances the mixing process and retards the demixing rate. Youngs (1992b) found that the incorporation of a disordered type of diffusion, calculated by means of a transport equation for turbulent kinetic energy, $\mathrm{K}$, was necessary to enhance the mixing rates and also slow down the rate of demixing for acceleration reversal problems (personal comm. 1993). Instead of the diffusive terms in the $\alpha$ equations, Youngs incorporated a turbulent diffusion and uses $v_{t}=0.09 \mathrm{~S} \sqrt{\mathrm{K}}$ to represent the physical instabilities. It appears possible to remove all of the numerical instabilities of the difference equations and yet retain all of the physical instability in this simple model. Travis, et al. (1976) have shown that the incorporation of a dissipative process, represented by momentum diffusion terms, does not remove the physical instability but only serves to damp out the growth rates for the very high wave number perturbations. The addition of artificial viscosity in the momentum equations was tried in our calculations, but numerical instabilities persisted. Here we use artificial diffusion of species added to the $\alpha$ equations in order to damp out numerical instabilities, and after some experimentation we have observed that the best compromise is achieved with $\sigma$ set to 0.1 . This has allowed for the identification of real physical instabilities that may occur in the calculations with minimal numerical instability to cloud the results. Species diffusion not only mitigates numerical instabilities, but it also serves as a crude representation of the physically real enhancement of 
species transport due to the disordered part of the species interpenetration. Youngs (1992b) has carried out a more thorough study of these matters via the addition of a transport equation for $\mathrm{K}$, from which a better representation of the diffusion of species can be extracted.

To achieve self-similarity, it is necessary to include an evolving length scale, S. For the calculations of this section, the scale is set proportional to the width of the mixing layer which also evolves with time, see Eq. (4.3.6). The proper choice for $\beta$ is somewhat uncertain. For this demonstration, $\beta$ was set to 0.3 in order to approximate the relative scale sizes that are observable from photographs of the TMZ of the Rayleigh-Taylor instability as reported by Smeeton and Youngs (1987). Actually, the two parameters, $C_{D}$ and $\beta$, can be reduced to one parameter since they only appear as a ratio in the momentum equations. We find that reasonable agreement with experiment occurs when this ratio is kept near 40 .

To demonstrate the capabilities of the above set of two-field equations, we show how they behave in a one-dimensional calculation of both mixing and demixing circumstances. The ability to demix is relatively straight forward for the two-field equations, and it is for this reason that the two-field equations sometimes find favor with researchers over the singlepoint turbulence transport equations for modeling circumstances with variations in acceleration. The plots will be given as functions of $y$, the distance across the TMZ, time, and the self-similarity variable $X$, where $X=(A t) * g * t^{2}$.

\subsection{A Mixing}

For the numerical results of the two-field equations applied to a mixing circumstance, refer to Fig. 4.3.1 through Fig. 4.3.3. These results are for the incompressible case of a heavy fluid, $\rho=2.0$, sitting over a lighter fluid, $\rho=1.0$, with an acceleration of -1.0 applied to the system in such a way as to initiate mixing, i.e., the extended Rayleigh-Taylor instability. In all of the plots, the configuration just described corresponds to the lighter fluid occupying the region of $y<0$ and the heavier fluid occupying the region of $y>0$. For the mixing case, the acceleration vector is pointing from top to bottom, which establishes a pressure gradient in 
the fluid that is consistent with the highest pressure residing in the lighter fluid. The calculation is carried out to a non-dimensionalized time of 55 in order to show the earliest manifestations of physical instability inherent in the two-field equations. As discussed above, the modified equations are only capable of describing ordered interpenetration and small departures from this. The differential equations are unstable in the presence of a relative velocity for any level of viscosity. Viscosity renders the equations well-posed, but nevertheless nominal levels of viscosity leave the perturbation growth rate relatively unchanged except at high wave numbers. The physical process represented by the real differential instability that appears in the calculations near $t=50$ is identified as early stages in the transition to a turbulent flow.

When referring to the plots we use the words "bubble" and "spike" to characterize the configuration of the two interpenetrating fluids. The word "bubble" is used to define the configuration of the lighter fluid as it penetrates the heavier fluid, and the word "spike" is used to define the configuration of the heavy fluid as it penetrates the lighter fluid. It is understood that only in the earliest stages of the Rayleigh-Taylor mixing does the configuration resemble anything close to bubbles and spikes, and the later time behavior of the two fluids can only be characterized as a turbulent or chaotic flow. For lack of a more descriptive terminology, we will use the words "bubble" and "spike" to describe the two interpenetrating fluids for the late stages of the mixing process.

The plots of Fig. 4.3.1 are evolving in a self-similar fashion. This self-similarity is an important feature to these types of flows and has been observed experimentally for RayleighTaylor type mixing problems (Smeeton \& Youngs, 1987; Burrows, et al., 1984; Read, 1984). In Chapters 3 and 4, extensive use will be made of the fact that this turbulent flow develops in a self-similar fashion. A feature that should be mentioned about Fig. 4.3.1 is the appearance of the instability for times near $t=50$. This is a manifestation of the physical instability present for the case of two interpenetrating fields. 


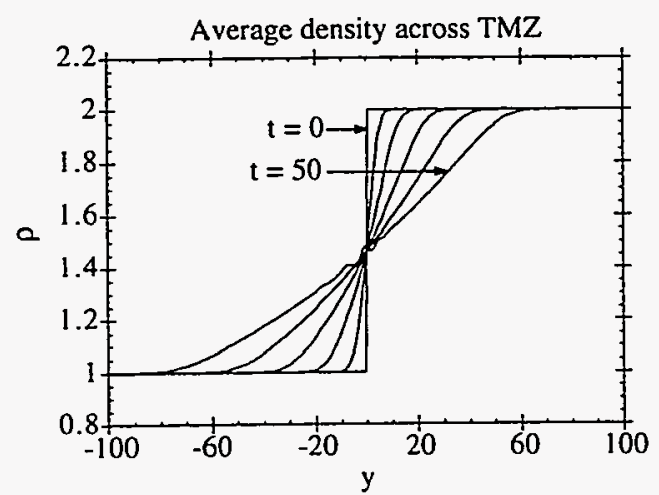

(a)

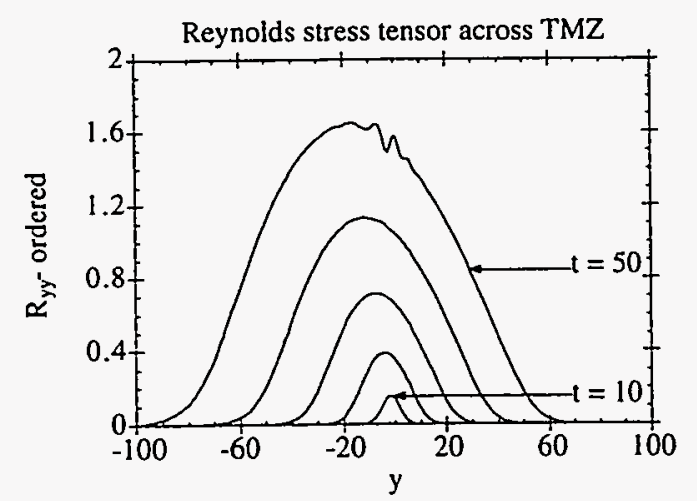

(c)

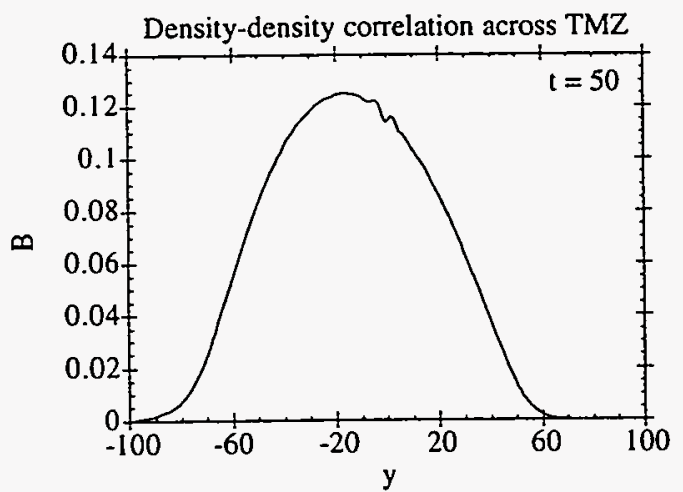

(e)

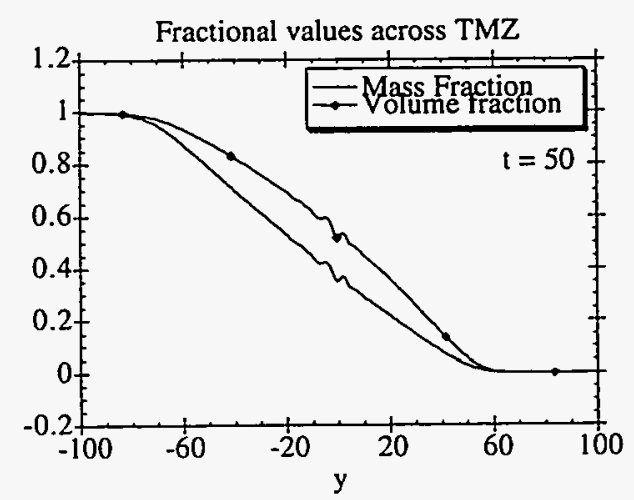

(b)

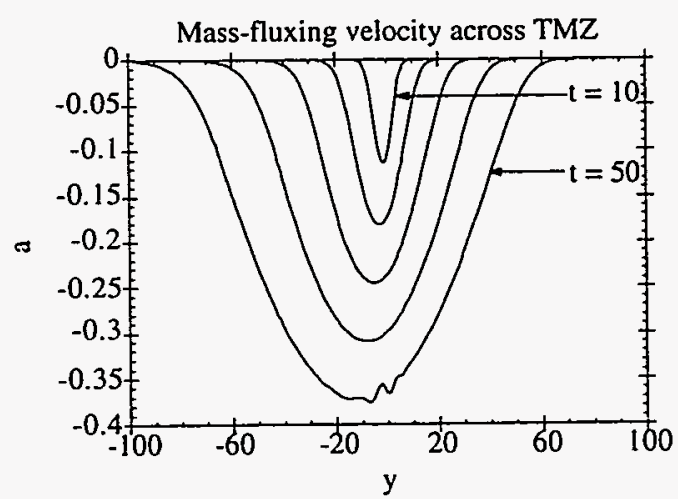

(d)

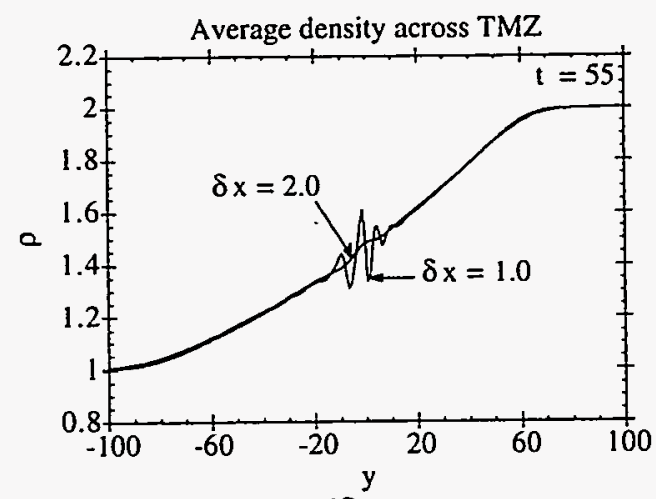

$(\mathrm{f})$

Figure 4.3.1. Examples of various two-field variables plotted across the TMZ throughout the mixing process of the Rayleigh-Taylor instability. Plots (a), (c) and (d) illustrate the time evolution of the variables by plotting a profile every 10 time units from a time of 0 to 50 . Plots (b) and (e) show the variables at a time of 50. Plot (f) illustrates the effects of cell size on the physical instability that is becoming significant by a time of 55 . 
As was stated earlier in this section, the diffusion coefficient, $\sigma$, was chosen so as to mitigate the deleterious effects of any numerical instabilities while preserving the capability of the code to describe the physical instability. Figure 4.3.1(f) is included to confirm that this is indeed a physical instability by showing that the instability growth rate increases for the finer grid $(\delta \mathrm{x}=1.0)$. This is consistent with the fact that for the finer grid, we are resolving the shorter wavelengths, i.e., the larger wave numbers, which are shown by stability analysis to have a larger growth rate of the perturbation amplitude. Plots of the Reynolds stress tensor, the mass-fluxing velocity, and the density-density self correlation are included for comparisons with the spectral transport results of Chapter 3.

The principal feature that should be pointed out is the excellent agreement with experiment. The plots of Fig. 4.3.1 show the general behavior of the TMZ for the configuration described above, but in order to compare with experiment we will use a plot of the volume fraction when the TMZ is approximately $100 \mathrm{~mm}$ wide for densities and an acceleration that match exp. \#94 of Smeeton and Youngs (1987). Figure 4.3.2 shows this comparison with data taken from exp. \#94 at $\mathrm{t}=79.4 \mathrm{~ms}$. For this experiment, calcium chloride solution $\left(\rho=1.142 \mathrm{~g} / \mathrm{cm}^{3}\right)$ and hexane $\left(\rho=0.66 \mathrm{~g} / \mathrm{cm}^{3}\right)$ are the two fluids. The two-fluid configuration is accelerated at $43 \mathrm{~g}_{0}$, where $\mathrm{g}_{0}=980 \mathrm{~cm} / \mathrm{s}^{2}$. This set of data is taken for comparison because it represents a typical TMZ structure found in results for this type of mixing experiment once the TMZ has reached the self-similar stage of development. Once this occurs the width of the data sets can be rescaled so as to coincide with other sets of data. For this reason, the basic feature to note is the agreement between the structure of the two data profiles of volume fraction within the TMZ of Fig. 4.3.2. The bubble interpenetration depth is also compared between this model and experiment. For this experiment, Youngs reports a value of 0.051 for the self-similarity coefficient, $\alpha$, i.e., the slope of the line describing the bubble penetration depth when plotted as a function of X. From our numerical calculation, we find a value of 0.055 for $\alpha$. 


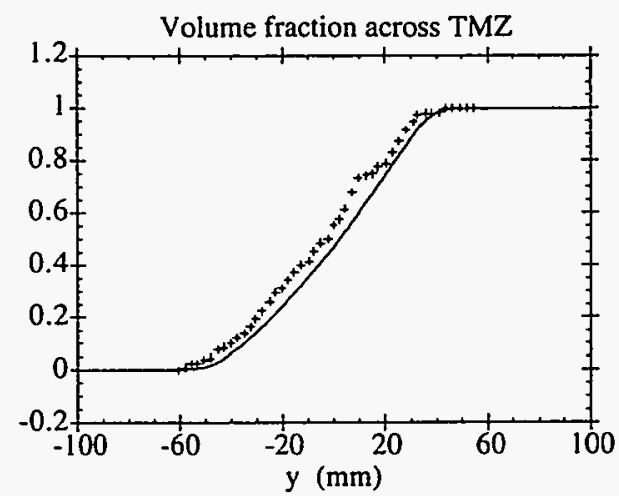

Figure 4.3.2. Comparison to experiment of the volume fraction of the top fluid for a mixing calculation. The solid line is the numerical results and the symbols are data from Fig. 25 (exp. \#94) of Smeeton and Youngs (1987).

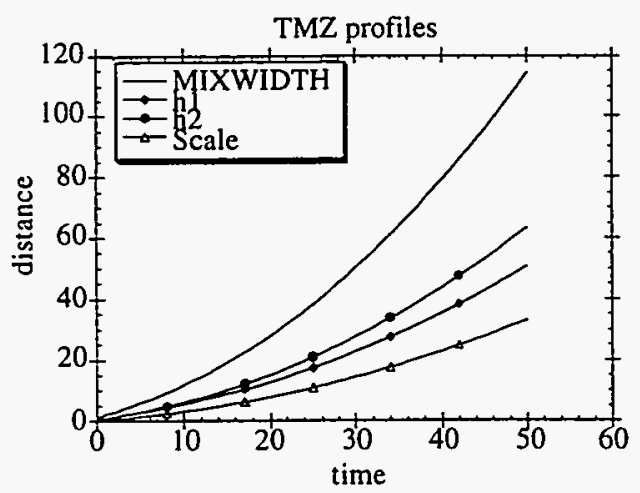

(a)

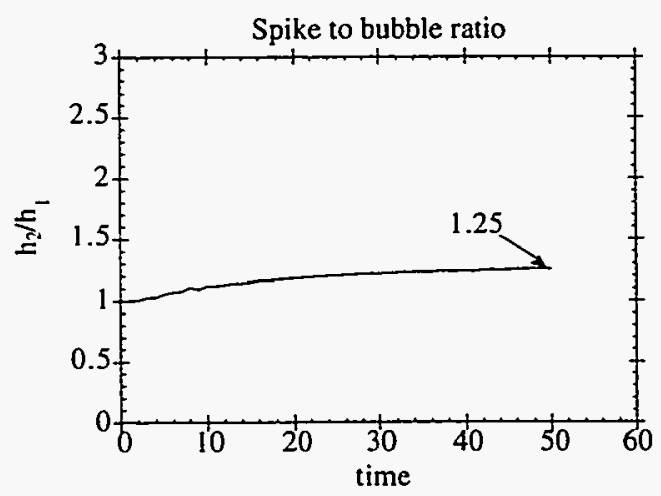

(c)

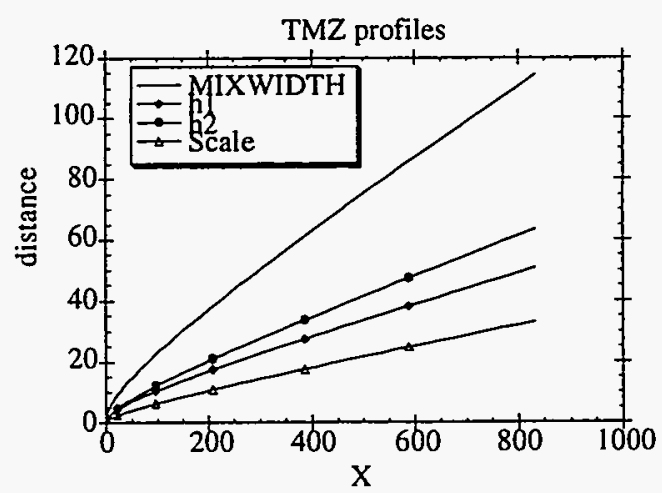

(b)

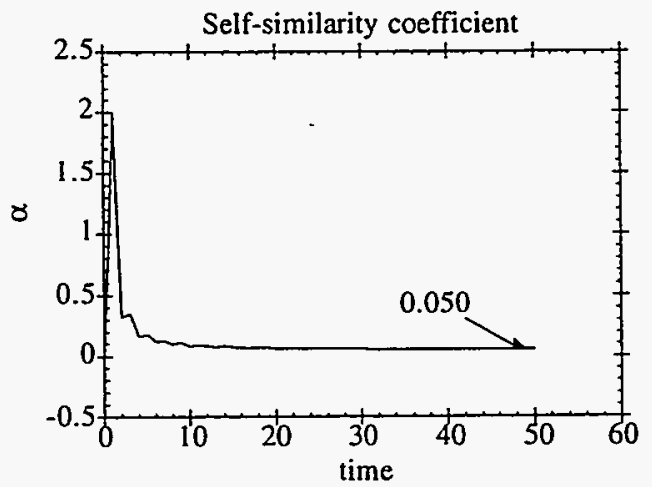

(d)

Figure 4.3.3. Plots (a) and (b) illustrate the evolution of the penetration depth for the bubbles, $h_{1}$, spikes, $h_{2}$, the overall width of the TMZ, and the scale that is calculated for these equations. Note, the profiles grow quadratically in time in plot (a) and linearly in X in plot (b). Plot (c) shows the spike to bubble ratio, which has leveled off to 1.25 , and plot (d) shows the self-similarity coefficient, $\alpha$, which is the value of the slope of the $h_{1}$ profile in plot (b). 
Upon examination of Fig. 4.3.3, it is evident that the flow is close to self-similarity by $t=30$. We can identify this behavior by the linear variation of the TMZ profiles when plotted against the self-similarity variable, $X$. When the flow attains self-similarity, all of the flow variables change in constant proportion to one another; hence, the spike to bubble ratio maintains a constant value of 1.25 which is consistent with the experimental observations of Smeeton and Youngs (1987). A self-similar value of $\alpha=0.050$ for the coefficient is also consistent with the value that Youngs reports from his experiments (1987), and calculations when using a two-field model (1992b) to simulate this same flow.

\subsection{B Demixing}

The second example is the application of the above two-field equations to a demixing of the TMZ due to a reversal of the acceleration field. The results of this calculation are presented in Figs. 4.3.5 - 4.3.7. For the purpose of this illustration, we first mix the layer as described above. At a time of $t=30$, (the time at which the flow has become approximately self-similar), the acceleration is reversed so as to created a pressure gradient in the opposite direction.

Numerical simulation of demixing is a greater challenge than mixing. When demixing occurs, pure fluid situated immediately next to the TMZ is receiving a flux of droplets of its own kind. These droplets could, in physical reality, penetrate into the depths of its own kind of pure fluid, possibly with significant splash, and even with entrainment of the other material. In our two-field representation, however, the possibility of a statistical component to the velocity for each material at a given height is precluded; $u_{1}$ and $u_{2}$ describe only a single velocity, for which there is no fluctuating part. For the numerical calculations reported here this constraint implies that a returning droplet comes instantly to rest when it encounters the current pure-fluid interface. Consider, for example, the lower fluid, \#1 of Fig. 4.3.4. When cell \#j attains (or exceeds) $\alpha_{1}=1.0$, the value of $\alpha_{1}$ in that cell is set equal to 1.0 , and we also set $\alpha_{2}=0.0$. 


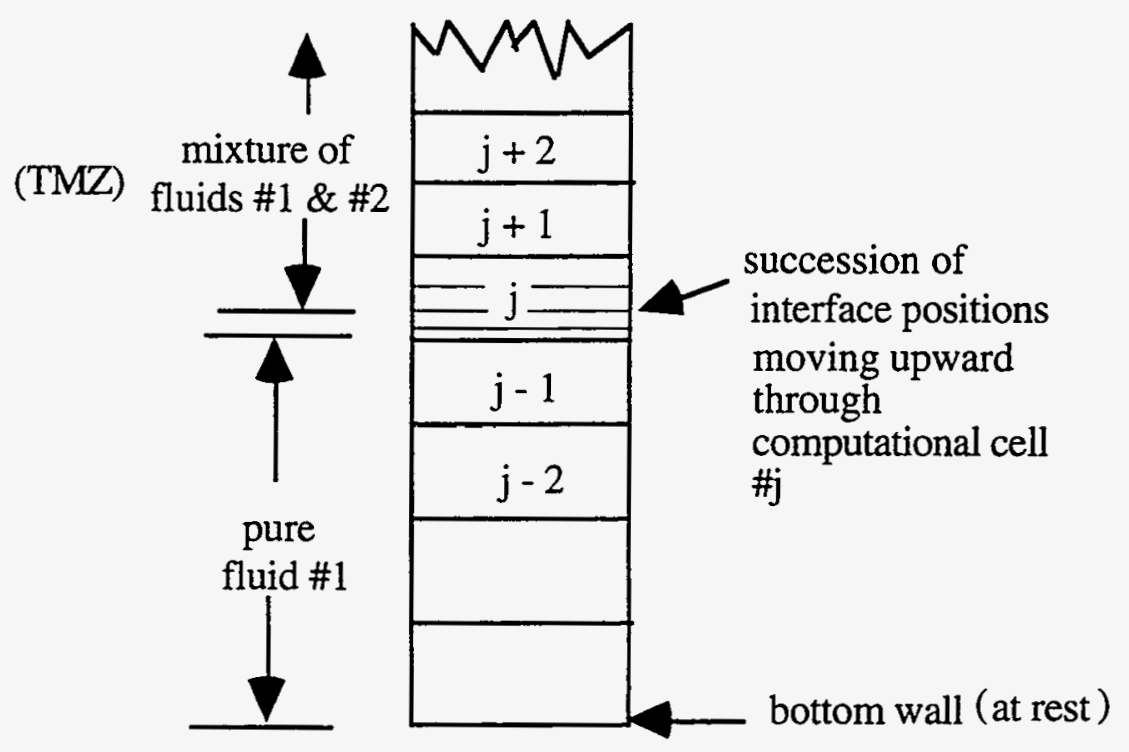

Figure 4.3.4. Schematic of the computational grid. Pure fluid \#1 is below the TMZ, which is comprised of both fluids. The lower edge of the TMZ is located in cell $\mathrm{\# j}$.

(With sufficiently small time step per calculational cycle, the effects due to the violation of the conservation of mass from this process can be made as small as desired). Incompressibility forces $\alpha_{1} \mathrm{u}_{1}+\alpha_{2} \mathrm{u}_{2}=0$ in general, so that when $\alpha_{1}=1.0$, the immediate effect is $u_{1}=0$. Thus this technique destroys kinetic energy, which is instantaneously converted to heat in this highly dissipative model. As described elsewhere, the turbulence transport description (single-point of Chapter 2 or spectral of Chapter 3 ) is capable of representing more general interactions between pure fluid and the returning droplets, for which a more careful accomplishment of the dissipation is required.

The plots of Fig. 4.3.5 are laid out in such a way so as to illustrate the demixing due to the acceleration reversal at $t=30$. The left column shows evolution profiles for the density, Reynolds stress (ordered component), and $B$ up to the time of $t=30$. The right column shows the demixing due to the acceleration reversal that occurs at $t=30$. The plots are given from $t=30$ through $t=50$, incrementing in steps of $\Delta t=5$. The density plot of Fig. 4.3.5(b) illustrates the demixing of the TMZ back to an almost completely demixed initial configuration by a time of $t=50$. 


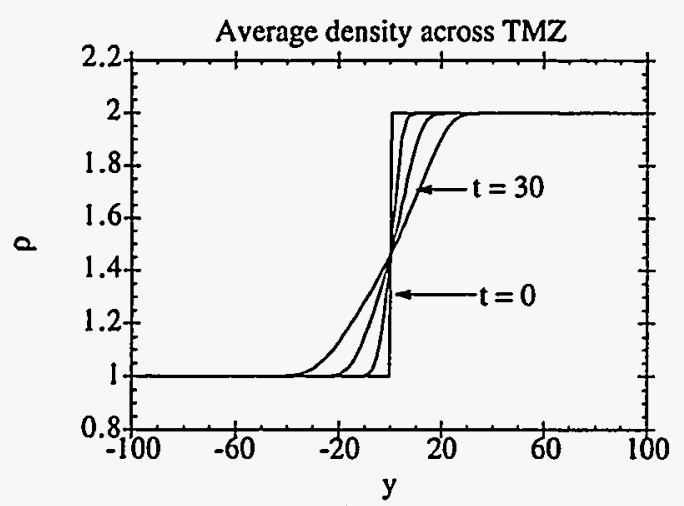

(a)

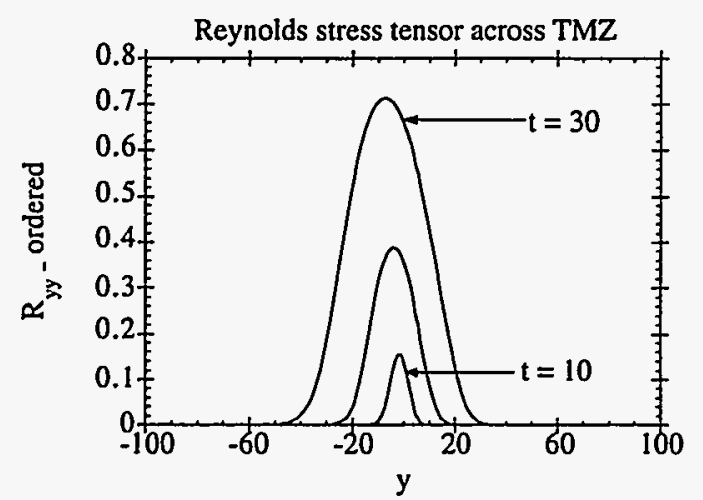

(c)

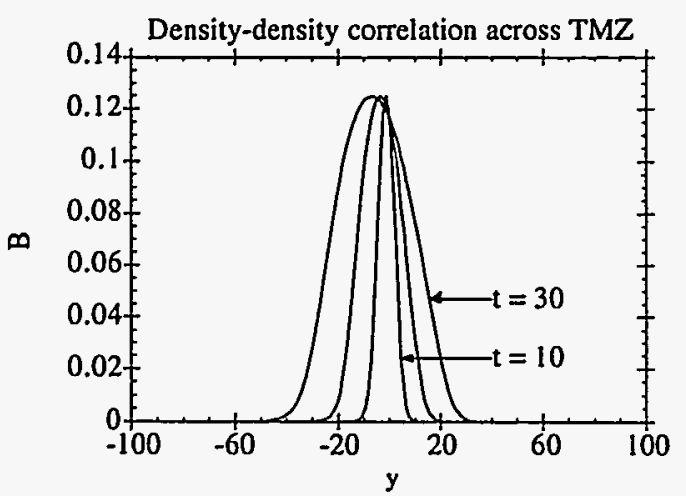

(e)

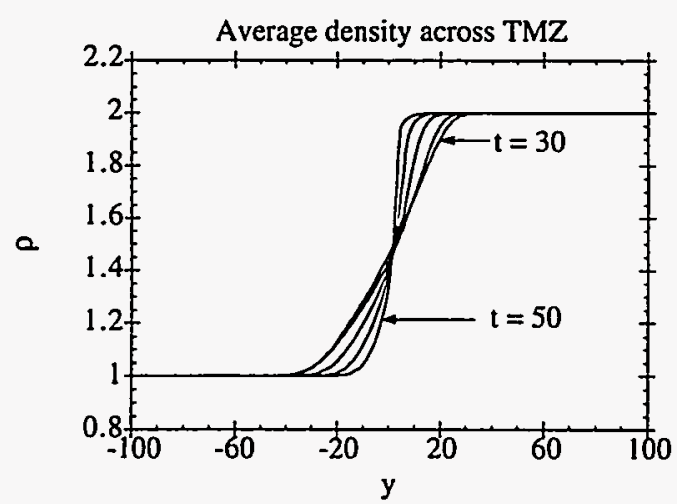

(b)

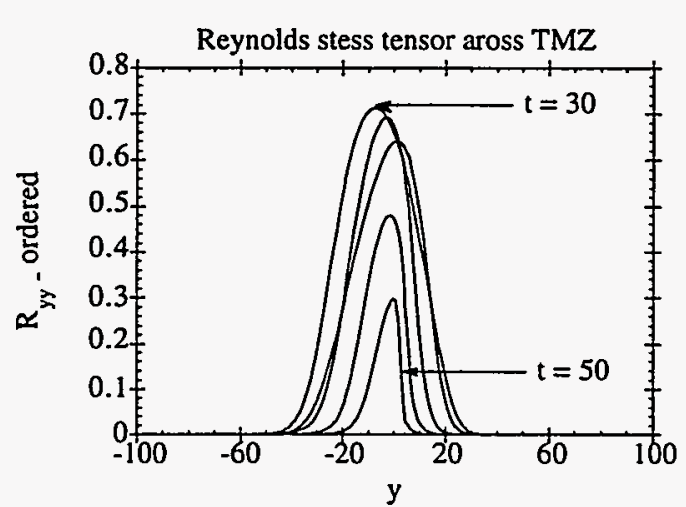

(d)

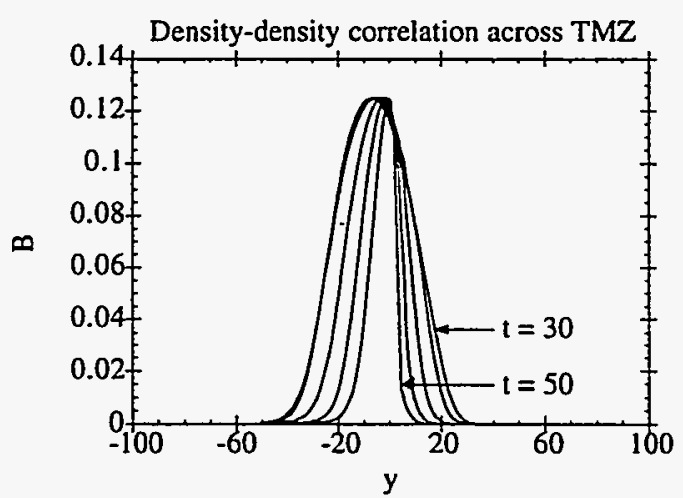

(f)

Figure 4.3.5. Plots (a), (c), and (e) show the variable profiles through a time of 30 , which corresponds to the time at which the acceleration is reversed, and plots (b), (d), and (f) illustrate the demixing that results from the acceleration reversal. 


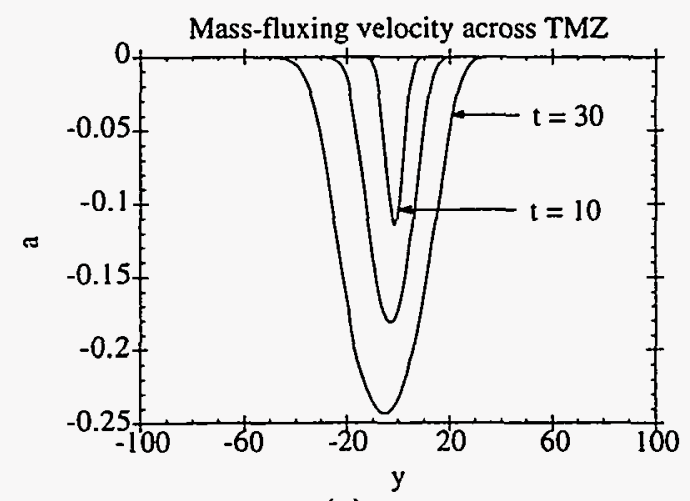

(a)

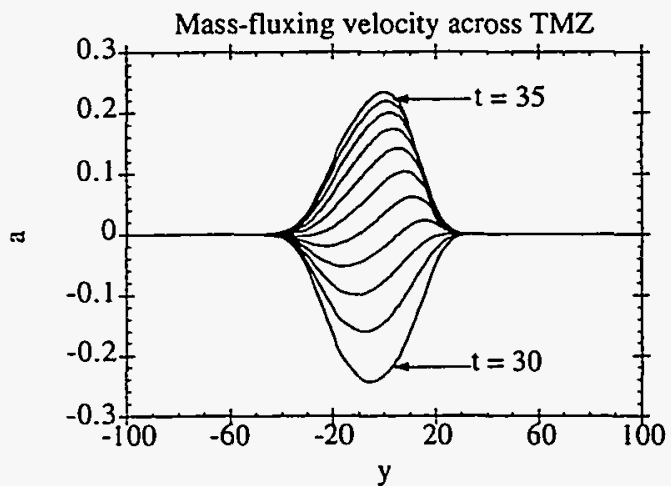

(b)

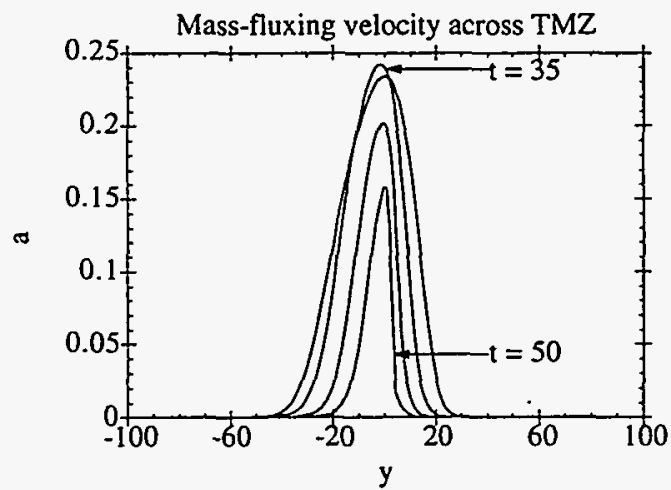

(c)

Figure 4.3.6. Mass-fluxing velocity profiles for (a) times of 10,20 , and 30 , to show mixing, (b) times of every 0.5 from 30 through 35 , to show the reversal due to the acceleration reversal at $t=30$, and (c) times of every 5 from 35 through 50, to show the decrease in velocity due to the diminishing TMZ.

Figure 4.3.6 is included to show the effects of the acceleration reversal on the massfluxing velocity, a, which is defined and identified in Chapter 2. Figure 4.3.6(a) shows the increasing velocity associated with the mass fluxing due to the acceleration from $t=0$ through $t=30$. The overall mass-fluxing velocity is negative, which corresponds to the heavier fluid falling into the lighter and a net downward motion of the center of mass of the system. Figure 4.3.6(b) shows the manner in which the velocity reverses in response to the acceleration reversal. Note that the velocity reverses in an asymmetric manner across the mixing layer. This is consistent with the expectation that bubbles of lighter fluid will reverse more quickly than spikes of heavier fluid due to inertial effects. This evolution of the massfluxing velocity shown in Fig. 4.3.6(b) bears significance on Chapters 3 and 4 . Notice that these profiles are not self-similar. That is to say, if we were to modulate all of the profiles 
with the maximum amplitude, they would not overlie one another. This is a circumstance (rapid transients) in which the single-point models break down. In Chapter 7 the lack of selfsimilar persistence of the spectral shapes for the mass-fluxing velocity, a, through a rapid transient will be discussed and compared in order to verify the loss of self-similarity of the amplitude-modulated profiles observed in Fig. 4.3.6(b). In Fig. 4.3.6(c), the profiles for $\mathrm{t}=35$ through $\mathrm{t}=50$ decrease as expected.

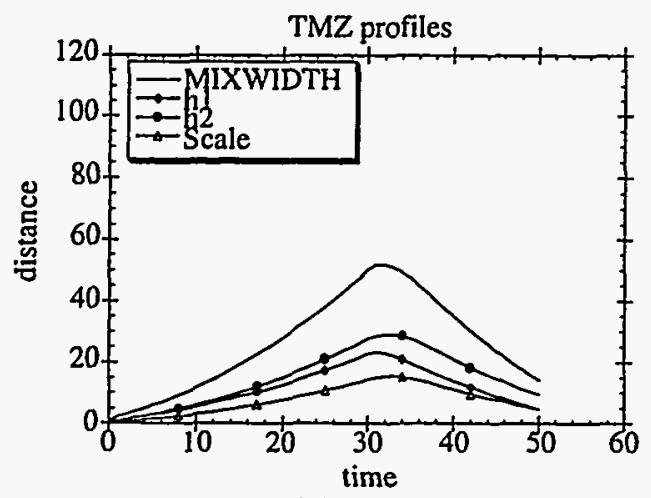

(a)

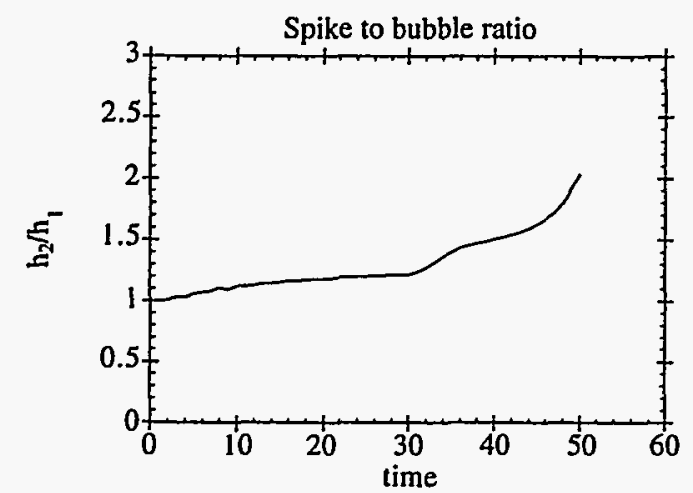

(b)

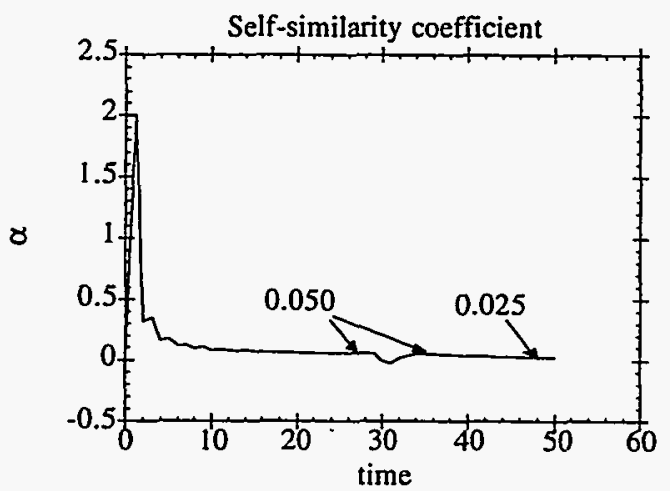

(c)

Figure 4.3.7. Time evolution of the (a) width of the TMZ, amplitude of the scale of the flow, and the depth of penetration of the spikes, $h_{2}$, and the bubbles, $h_{1}$, (b) spike to bubble ratio, and (c) self-similarity coefficient for the entire mixing and demixing process.

Figure 4.3.7 shows the expected growth of the TMZ during the acceleration phase and the resulting shrinkage of the TMZ due to the acceleration reversal. Note that the spike to bubble ratio grows more quickly after the acceleration reversal due to the inertial effects that were mentioned in the discussion of Fig. 4.3.6. One more feature that can be attributed to the inertia of the heavy fluid and is interesting to comment on is the dip in the self-similarity co- 
efficient of Fig. 4.3.7(c) at the time of the acceleration reversal, $t=30$. Since $\mathrm{h}_{1}=\alpha^{*} \mathrm{At}{ }^{*} \mathrm{~g}^{*} \mathrm{t}^{2}$, we can express the velocity of the bubble penetration as $\dot{\mathrm{h}}_{1}=2 * \alpha * A t^{*} \mathrm{~g} * \mathrm{t}$. The value for $\alpha$ is calculated by dividing the bubble velocity by $2 * \mathrm{At} * \mathrm{~g} *$. This denominator instaneously becomes negative upon acceleration reversal from a value of +1.0 to -1.0 at $t=30$. Due to the inertia of the fluid, there is a finite amount of time involved with reversing the bubble velocity from its positive value at $t=30$ to the negative value associated with the shrinking of the TMZ (demixing). Hence, the dip to negative values for $\alpha$. If the calculation had been run with a smaller time step, a much sharper dip to negative values would be observed.

Another interesting feature of the plot of Fig. 4.3.7(c) is the return of $\alpha$ to approximately the same value as before the acceleration reversal. We do not expect the system to asymptote to a self-similar state for the demixing circumstance due to the introduction of the additional dimension, the width of the TMZ at the time of the acceleration reversal, but the fact that the mixing and the demixing rates are practically the same is what motivated Youngs (1992b) to add some "disordered" turbulence to his two-fluid approach in the first place. This feature increases the mixing and inhibits the de-mixing.

\subsection{Summary of the two-field approach}

The two-field equations are discussed and applied to the mixing of two fluids via the late stages of the Rayleigh-Taylor instability. These equations were discussed to demonstrate

the following ideas: (1) the two-field equations can be made to match experimental data upon implementation of enhanced drag terms and an evolving length scale that is somewhat arbitrary in its origin, (an evolving length scale is necessary for the attainment of selfsimilarity) and (2) the two-field equations can be made to describe de-mixing once careful attention is paid to the region where mixed fluids fall back into the pure fluid and proper dissipation methods are included in the numerics. 
The above comments nicely motivate the need for the development of the spectral model discussed in Chapter 5. The desire to provide a basis upon which to formulate a relevant length scale of the flow provides significant motivation for the development of the spectral model. Also, ideas about the drag terms of this two-field model are extended to the formulation of the spectral model.

An issue that deserves comment here is the logic behind using the single-point turbulence transport equations as the basis upon which to build the spectral model of Chapter 5 . After reviewing the behavior of both the single-point turbulence transport approach of Chapter 3 and the two-phase flow equations of this chapter, one might be inclined to designate the two-phase flow equations as the basis for further investigations. A key issue in deciding which modeling technique to follow is the proper identification of the components of a variable density fluid. As two fluids mix down to the molecular levels it becomes increasingly difficult to identify the original components. Yet it is very simple to identify fluctuations in the density as a result of this mixing. Considerations such as this have prompted us to develop the spectral model of Chapter 5 from a single-field approach as opposed to a two-field approach. 


\section{The Variable Density Spectral Approach}

\subsection{Introduction}

For two or more interacting fluids, the usual approach in describing their interpenetration has been through the use of two-field or multifield equations, as discussed above. More novel is to consider the problem in terms of a single field of variables in which there are large variations in fluid density. [Single-point models have been described, and applied with considerable success, by Andronov, et al. (1982), by Nikiforov (1991), and by Besnard et al. (1987).] Two-point models are only recently being investigated, notably by Clark and Spitz (1995), Godeford and Cambon (1994), for homogeneous circumstances, and in this current work for inhomogeneous configurations.

In this study we obtain correlations that are functions of two points in space. We pass to wave-vector space by means of Fourier transforms of the separation variable of the two points. Due to the complex nature of the transformed governing equations, we angularly integrate these equations in $\mathbf{k}$-space to reduce the spectral dependence to $|\mathbf{k}|$. This transformation allows for a convenient identification of length scales of Fourier modes. We realize that this simplification captures only the real part and not the imaginary part of the spectrum, and that a significant portion of the physics is averaged out of the equations. For now, however, we feel that a fully three-dimensional $\mathbf{k}$-space model is unwarranted due to lack of any experimental verification.

In the current form of our representations, we are not especially concerned with that part of the spectrum that is associated with density discontinuities and is richly filled with the high-wave-number components. Its existence requires consideration, possibly through boundary conditions at high-wave-numbers in k-space, but for our purposes the dominant spectral structure is associated with relatively large clouds or clumps of a fine-scale dispersed phase or with large-scale coalescent structures. Thus we ignore for now the interesting 
questions that can arise with a large Schmidt number, in which the $\mathrm{k}^{-1}$ "passive-scalar" behavior of imbedded material in a carrier fluid extends well beyond the wave numbers for which viscosity effects dominate the dynamics of the $\mathrm{R}_{\mathrm{ij}}$ spectrum.

Building on the analytical foundations of Clark and Spitz (1995), we incorporate much guidance from two-field formulations and from many of the constant-density developments, both single-point and spectral. These relationships are discussed more fully in the next section, which describes the equations and the origins of contributing terms.

It will be noted that the equations for our current formulation have already been angularly averaged in (wave vector) $\mathbf{k}$-space so that the variables are functions of $k \equiv|k|$ (wave number as well as $\mathbf{x}$ and $\mathrm{t}$ ). Moreover, for clarity of description we give them first as functions as $\mathrm{k}, \mathrm{y}, \mathrm{t}$, only, as specialized to a free mixing layer of infinite extent in the $\mathrm{x}$ and $\mathrm{z}$ directions and accordingly the ensemble averages varying spatially only in the y direction.

As with single-point turbulence transport modeling, there are many powerful constraints that useful spectral equations must include. Some of these are discussed in Sections 5.2 and 5.3. The list includes

- conservation of mass, momentum, and energy in both physical and wave-number space;

- proper tensor formulation;

- same free indices

- same symmetries

- proper tensor properties: modeled terms in comparison with unmodeled terms;

- dimensional consistency;

- Galilean invariance;

- integrability (over wave numbers) to reasonable single-point forms;

- in absence of further guidance, simplicity, manifested by omission of certain products of first derivatives and of higher derivatives than second (in the spirit of the Stokes stress tensor in the Navier-Stokes equations); 
- realizability, more generally that the modeling obeys some (as yet unformulated) principal resembling the second law of thermodynamics.

These constraints help us to achieve meaningful formulations. They may lead to simple low-order approximations (e.g., for cascade in wave-number space) that capture much of the physics of what is happening, both qualitatively and quantitatively, as illustrated by the test examples described in this report.

To summarize the motivations for developing a tractable and broadly applicable spectral formulation for turbulence in a fluid with variable density, we have mentioned its relationship to the enhancement of single-point models. In Section 1.1 it was pointed out that a transport equation for the dissipation tensor must be derived. For this endeavor, the knowledge of the scales associated with the turbulence proves very useful for formulating credible turbulence transport equations. The spectral formulation described in this report provides a basis on which to extract scale information of the turbulence. In addition, the spectral studies go much further in delivering both theoretical and practical value.

- They serve as a basis for understanding many properties of turbulence, for example anisotropic self-similarity.

- They show the limitations of single-point models in circumstances of rapid transients.

- They remove the constraints of assumed spectral equilibrium in situations of spectral transients.

- Frees the modeling from the simplistic characterization of turbulence by the magnitude of turbulence energy $(K)$ and a turbulent length scale $(L)$, or a dissipation rate $(\varepsilon)$.

- Leads to less drastic modeling assumptions.

- Not limited to certain regimes of Reynolds numbers.

- Hence, they allow a much greater scope of interesting problems to be solved realistically, albeit at greater computational expense, and allow the derivation of model equations that go beyond current one-point formulations in their applicability (as shown in Appendix E). 


\subsection{Formulation of the model equations}

Development of the spectral model equations is described schematically in Fig. 5.2.1. From the spectral formulation there radiate numerous directions for investigation as shown in Fig. 5.2.2.

The physical processes described by the spectral transport equations are of three types, advective, pressure-related, and viscous. For each there are several features that need representation.

- Advective processes

- mean-flow advective transport

- mean-flow source to $R_{\mathrm{ij}}$

- stochastic advective transport

- kinematics of mixing

- Pressure processes

- local

- differential acceleration of fluids

- momentum exchange (drag)

- nonlocal

- in physical space: mean-flow coupling, diffusion

- in wave-number space: cascade, return to isotropy

- Viscous processes

- diffusion

- dissipation to heat

— momentum exchange between fluids 


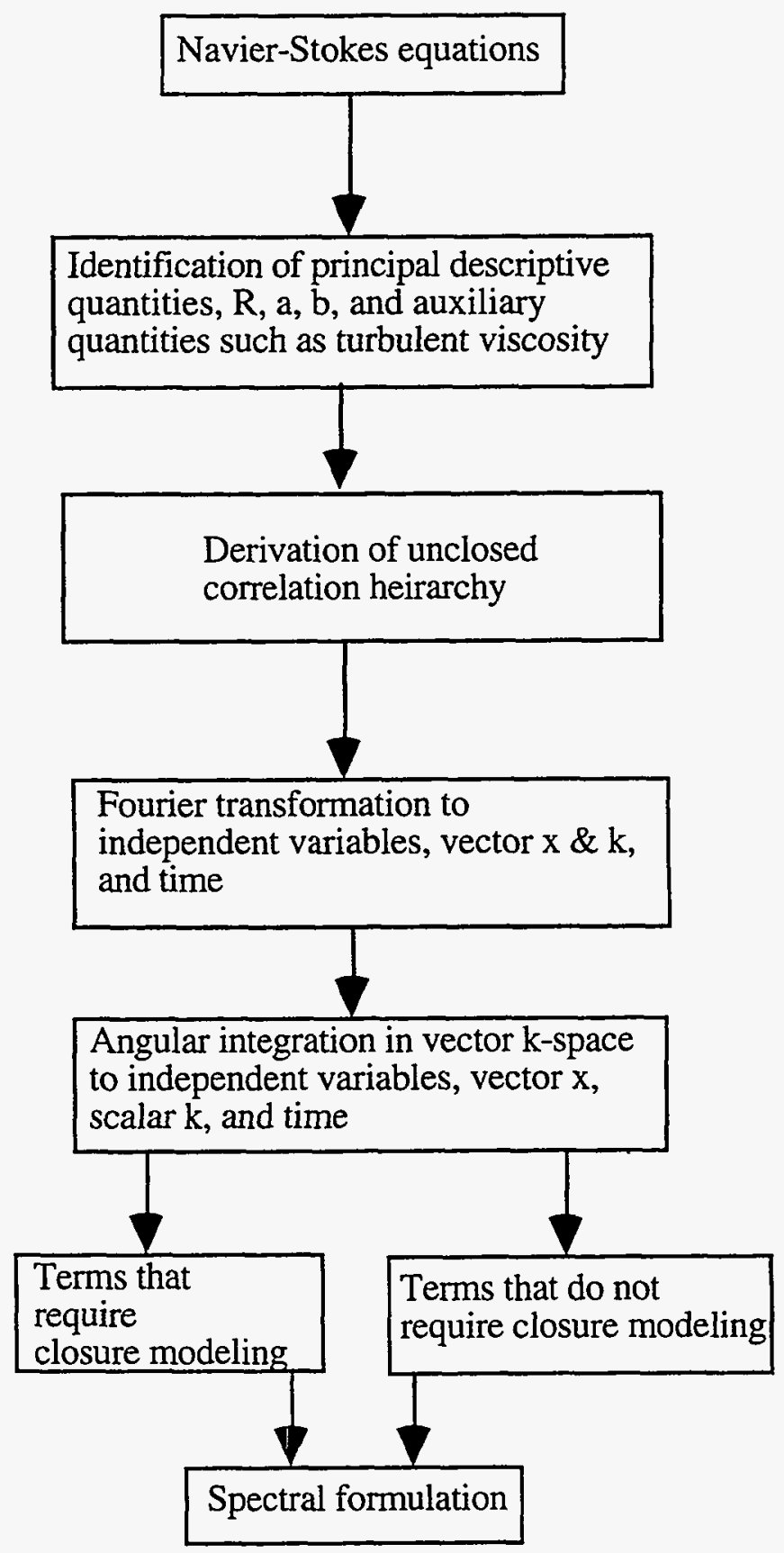

Figure 5.2.1. Flow chart describing steps of the spectral formulation. 


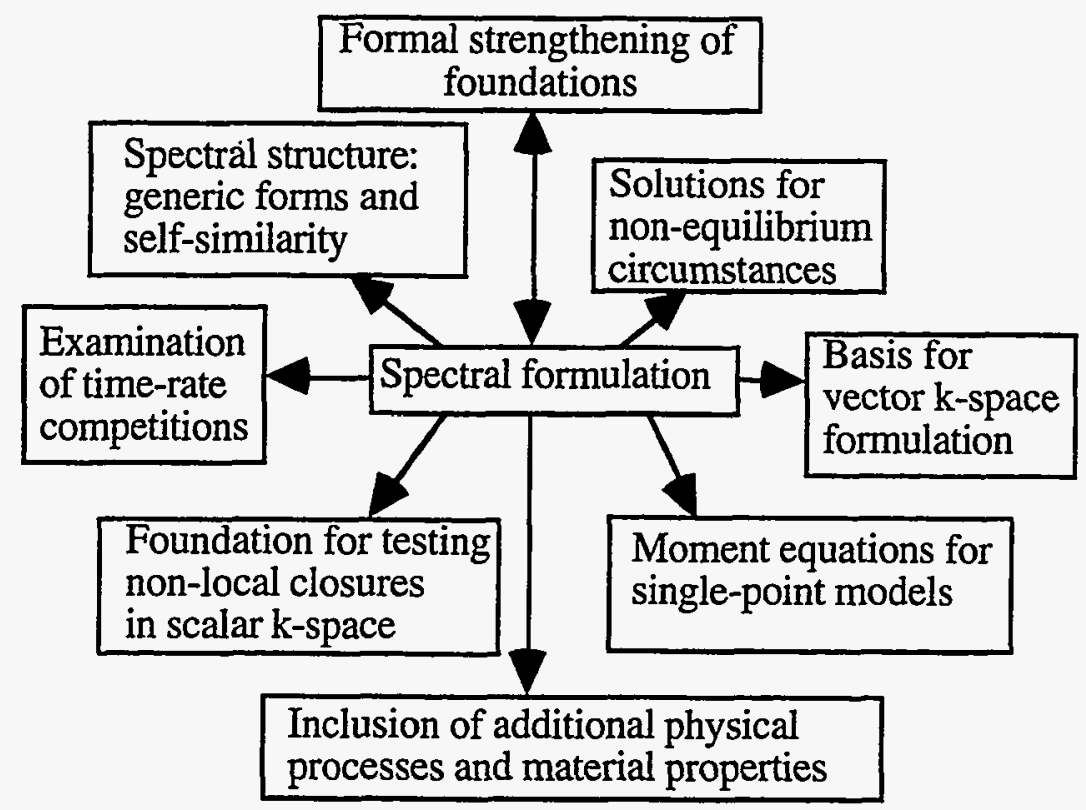

Figure 5.2.2. Flow chart describing all of the potential uses of a spectral formulation.

The representation of these processes is described in the discussion of terms for each of the transport equations. Some of them descend directly from the Navier-Stokes equations without the need for closure modeling. Others require the derivation or a postulate of closure modeling, for which some closure constraints were described in the previous section. As guidance for the development of closure modeling we further constrain our approach by means of identifying a one-to-one correspondence between turbulent processes and the individually modeled terms. Although, for example, the processes of return to isotropy, diffusion, and cascade in wave-number space all have their origins in pressure-velocity correlations, we employ separate terms for their effects with neither allowed to represent any part of the other. In this manner we intend to capture the dominant effects of each without the higher-order complexity of combined effects representation. Several other instances are mentioned in the descriptive text for each of the terms that have required modeling.

Wherever possible, closure modeling has been guided by extension of the procedures employed by previous investigators for single-point (nonspectral) closure.

With all of these constraints, both hard and soft, there remains surprisingly little flexibility in the formulation, so it should not be unexpected that applications to real-world 
circumstances exhibit a good degree of validity. The principal areas that remain for extension lie in nonlocal processes in wave-number space. These extensions for the variable-density formulation will likely result in additional tightening of agreement between calculations and experiments.

Although this discussion focuses on closure modeling by means of severely constrained postulates, there are several partially-derivational techniques that can be employed. Two examples are the following:

- Closures for an $\mathrm{N}^{\text {th }}$-order correlation are often accomplished through the derivation of its transport equation, the appending of a dissipation term proportional to the correlation, the neglect of both time variations and advection, and a very simple

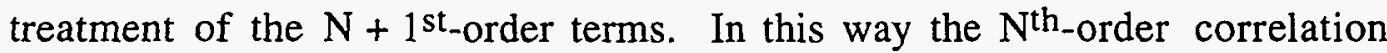
emerges as a purely algebraic expression of the lower-order quantities. The famous Boussinesq approximation of the Reynolds stress tensor as discussed by Hinze (1987, page 23) can be obtained in this fashion, and gradient-flux expressions for a variety of closures have likewise been derived (Harlow, 1968; Daly \& Harlow, 1970;.Schubauer \& Tchen, 1959).

- A useful closure guidance comes from the idea that the larger-scale part of the turbulence spectrum can be temporarily viewed as mean flow. Source terms for the small-scale part of the turbulence are then modeled from the "mean-flow" driving terms, and the loss from the large-scale parts is equated to the gain to the smallscale part. The next step is to return to considering the entire structure of scales to be turbulence and thus to use the derived source-sink model as a representation of cascade flux through wave-number space. 


\subsection{The spectral equations}

Following the usual convention of single-point turbulence modeling of variable density flows, the flow field variables, $\rho, \mathbf{u}$, and $p$, are decomposed into their mean and fluctuating parts and substituted into the conservation equations. The decompositions are

and

$$
\begin{aligned}
& \rho=\bar{\rho}+\rho^{\prime}, \\
& \mathbf{u}=\overline{\mathbf{u}}+\mathbf{u}^{\prime}, \\
& p=\bar{p}+p^{\prime},
\end{aligned}
$$

where the overbar denotes the non-mass-weighted ensemble average, and the prime denotes a fluctuation about the average with the average of a fluctuating quantity equal to zero. For variable density flows, it is useful to incorporate the mass-weighted averaging procedure introduced by Favre that leads to a conservative form of the Reynolds stress tensor, $R_{\mathrm{ij}}$, in the averaged momentum equations, where $R_{\mathrm{ij}}(\mathbf{x}, \mathrm{t})=\overline{\rho(x) \mathbf{u}^{\prime \prime}(\mathbf{x}) \mathbf{u}^{\prime \prime}(\mathbf{x})}$. The averaged massweighted velocity, $\tilde{\mathbf{u}}$, is defined as

$$
\tilde{\mathbf{u}}=\frac{\overline{\rho \mathbf{u}}}{\bar{\rho}},
$$

and $\mathbf{u}$ " denotes the mass-weighted fluctuation about this averaged quantity, such that $\mathbf{u}=\tilde{\mathbf{u}}+\mathbf{u}^{\prime \prime}$ with $\overline{\rho \mathbf{u}^{\prime \prime}}=0$.

There exists a strong motivation to identify a velocity associated with the average mass flux, $\overline{\rho u}$, since this quantity occurs in both the averaged conservation of momentum equation as well as the averaged conservation of mass equation. If we apply the Reynolds decomposition to this momentum density, $\overline{\mathrm{pu}}$, we get

$$
\overline{\rho u_{i}}=\overline{\left(\bar{\rho}+\rho^{\prime}\right)\left(\bar{u}_{i}+u_{i}^{\prime}\right)}=\overline{\rho u_{i}}+\overline{\rho^{\prime} u_{i}^{\prime}} .
$$

In order to identify a velocity that is responsible for fluxing the mass, we can use the Favre mass weighted velocity, $\tilde{\mathbf{u}}$. Thus we incorporate the mass weighted velocity into the expression for momentum density to get

$$
\tilde{u}_{i}=\bar{u}_{i}+\frac{\overline{\rho^{\prime} u_{i}^{\prime}}}{\bar{\rho}} .
$$


We define another important variable here, namely, the velocity a, associated with the net mass flux relative to the $\tilde{\mathbf{u}}$ frame of reference. From the above equation, we can see that this velocity associated with this mass flux is just

$$
a_{i}=\frac{\overline{\rho^{\prime} u_{i}^{\prime}}}{\bar{\rho}} .
$$

This is a very important quantity as far as this study is concerned. It is this quantity that describes the fluxing of mass relative to the $\overline{\mathbf{u}}$ frame of reference, and this idea is quantified by the resulting expression, namely,

$$
\tilde{\mathbf{u}}=\overline{\mathbf{u}}+\mathbf{a} .
$$

A transport equation for $\mathbf{a}$ is derived from the Navier-Stokes equations. This transport equation contains a source term, namely $b$, which couples with the mean pressure gradient, where $b=-\overline{\rho^{\prime}\left(\frac{1}{\rho}\right)^{\prime}}$. A transport equation for $b$ is derived from the conservation of mass equation.

Clark and Spitz (1995) give an extensive description of the foundations for two-point spectral representations of variable-density turbulence and describe the numerous challenges and issues associated with closure modeling for circumstances in which the distribution of one fluid throughout the other is purely homogeneous. Clark (personal communication, 1994) has subsequently proposed an extension for inhomogeneous distributions. The functions that he uses are similar to the single-point descriptors introduced in the BHR model. For two arbitrary points in space, $\mathbf{x}_{1}$ and $\mathbf{x}_{2}$, the two-point generalization of the single-point descriptors in the BHR model (Besnard, et al. 1987) are

$$
\begin{gathered}
R_{i j}\left(x_{1}, x_{2}\right)=\frac{1}{2} \overline{\left[\rho\left(x_{1}\right)+\rho\left(x_{2}\right)\right] u_{i}^{\prime}\left(x_{1}\right) u_{j}^{\prime \prime}\left(x_{2}\right)}, \\
a_{i}\left(x_{1}, x_{2}\right)=-\overline{u_{i}^{\prime \prime}\left(x_{1}\right) \rho\left(x_{1}\right) v\left(x_{2}\right)},
\end{gathered}
$$

and

where

$$
b\left(x_{1}, x_{2}\right)=-\overline{\rho^{\prime}\left(\mathbf{x}_{1}\right) v^{\prime}\left(\mathbf{x}_{2}\right)},
$$




$$
v(\mathbf{x})=\frac{1}{\rho(\mathbf{x})} .
$$

Note that there are other forms that could be used for these quantities, which also satisfy the requirement of reducing to the single-point form as $\mathbf{x}_{1} \rightarrow \mathbf{x}_{2}$ and possess the desired symmetries, i.e., those possessed by the constant density Reynolds stress tensor.

The constraints on the Reynolds stress tensor, as required by Clark and Spitz (1995) are

(1) $\mathrm{R}_{\mathrm{ij}}\left(\mathrm{x}_{1}, \mathrm{x}_{2}\right)=\mathrm{R}_{\mathrm{ji}}\left(\mathrm{x}_{2}, \mathrm{x}_{1}\right)$,

(2) As $\left|\mathbf{x}_{1}-\mathbf{x}_{2}\right| \rightarrow \infty, \mathrm{R}_{\mathrm{ij}}\left(\mathbf{x}_{1}, \mathbf{x}_{2}\right) \rightarrow 0$, and

(3) $\mathrm{R}_{\mathrm{ij}}\left(\mathrm{x}_{1}, \mathrm{x}_{2}\right)$ remain bounded as $\left|\mathrm{x}_{1}-\mathrm{x}_{2}\right| \rightarrow 0$.

There is no obvious constraint as to which choice is best except that whatever is chosen must be correctly transported by the Navier-Stokes equations. The choice is thus dictated more by properties of manipulative ease and transparency of interpretation.

Let us define

$$
\begin{aligned}
\mathbf{x} & \equiv \frac{1}{2}\left(\mathbf{x}_{1}+\mathbf{x}_{2}\right), \\
\mathbf{r} & \equiv \mathbf{x}_{1}-\mathbf{x}_{2},
\end{aligned}
$$

and substitute into the transported variables. We have for the additional Fourier transformations,

and

$$
\begin{aligned}
R_{i j}(x, k) & \equiv \int R_{i j}(x, r) e^{-i k \cdot r} d r \\
a_{i}(x, k) & \equiv \int a_{i}(x, r) e^{-i k \cdot r} d \mathbf{r},
\end{aligned}
$$

$$
\mathrm{b}(\mathbf{x}, \mathbf{k}) \equiv \int \mathrm{b}(\mathbf{x}, \mathbf{r}) \mathrm{e}^{-\mathbf{i k} \cdot \mathbf{r}} \mathrm{dr} .
$$

In this report, we work with variables and equations that have been angularly averaged in $\mathbf{k}$ space, that is,

$$
R_{i j}(x, k)=\int R_{i j}(x, k) \frac{k^{2} d \Omega_{k}}{(2 \pi)^{3}}
$$


and,

$$
a_{i}(x, k)=\int a_{i}(x, k) \frac{k^{2} d \Omega_{k}}{(2 \pi)^{3}}
$$

$$
\mathrm{b}(\mathbf{x}, \mathrm{k})=\int \mathrm{b}(\mathbf{x}, \mathbf{k}) \frac{\mathrm{k}^{2} \mathrm{~d} \Omega_{\mathrm{k}}}{(2 \pi)^{3}},
$$

where $\mathrm{d} \Omega_{\mathrm{k}}=\sin \theta \mathrm{d} \theta \mathrm{d} \phi$ for $0 \leq \theta \leq \pi ; 0 \leq \phi \leq 2 \pi$. From these we can recover the singlepoint forms,

$$
\begin{aligned}
R_{i j}(x) & =\int_{0}^{\infty} R_{i j}(x, k) d k \\
a_{i}(x) & =\int_{0}^{\infty} a_{i}(x, k) d k
\end{aligned}
$$

and,

$$
b(\mathbf{x})=\int_{0}^{\infty} b(\mathbf{x}, k) d k
$$

Clark and Spitz (1995) derive equations for a completely homogeneous configuration. For the inhomogeneous free mixing layer, we describe the necessary extensions in the most stripped-down form that we have found to give agreement with experiments. Thus we consider the special case of a mixing layer of infinite extent in the $\mathrm{x}$ and $\mathrm{z}$ directions, so that ensemble averages vary only with normal direction, $y$, scalar wave number, $k$, and time. For this configuration, the ensemble averages are equivalent to averages over each $\mathrm{x}-\mathrm{z}$ plane. The fluid is subjected to a body force, $\rho$ g, directed in the y direction. Below the mixing layer is a fluid with density $\rho_{1}$; above the layer is a different fluid with density $\rho_{2}$; for all of our calculations $\rho_{1}<\rho_{2}$, so that when $\mathbf{g}<0$ the configuration is unstable and mixing takes place. We also discuss the consequences of a reversal of the sign of $g$ after the mixing layer has become well developed. The fluid is confined by stationary boundaries far above and below the turbulent mixing zone (TMZ), so that there is nowhere any vertical volumetric flux and $\overline{\mathbf{u}}(\mathrm{y}, \mathrm{t}) \equiv 0$, so that $\tilde{\mathbf{u}}(\mathrm{y}, \mathrm{t}) \equiv \mathbf{a}$. 
The appropriate equations for $\bar{\rho}$ and $\tilde{\mathbf{u}}$ are the same as for the single-point formulation. The equations are presented here for one spatial dimension, with $\tilde{u}$ and a representing the $y-$ component of the respective velocity vectors;

and

$$
\begin{gathered}
\frac{\partial \bar{\rho}}{\partial t}+\frac{\partial \bar{\rho} \tilde{u}}{\partial y}=0, \\
\frac{\partial \bar{\rho} \tilde{u}}{\partial t}+\frac{\partial \bar{\rho} \tilde{u} \tilde{u}}{\partial y}=-\frac{\partial p}{\partial y}-\frac{\partial R_{y y}}{\partial y}+\bar{\rho} g,
\end{gathered}
$$

$$
\tilde{\mathbf{u}}=\mathbf{a} \text {. }
$$

The new equations for the spectral variables, $a(y, k, t), b(y, k, t), R_{n n}(y, k, t)$, and $R_{y y}(y, k, t)$ are presented in the following discussion, along with descriptions of the contributing terms in each. Assuming axisymmetry, so that $\mathrm{R}_{\mathrm{xx}}=\mathrm{R}_{\mathrm{zz}}$, the values for these quantities can be determined by knowing $R_{y y}$ and $R_{n n}$. For this configuration in principal coordinates, $\mathrm{R}_{\mathrm{ij}}$ has no off-diagonal components. With the addition of horizontal shear the additional terms are the same as those given by Besnard et al. (1990) in their analysis of a free shear, and equations for $R_{x y}$ and/or $R_{z y}$ are obtained as straightforward extensions.

For $a(y, k, t)$, we write

$$
\begin{aligned}
\frac{\partial \mathrm{a}}{\partial \mathrm{t}}= & -\tilde{\mathrm{u}} \frac{\partial \mathrm{a}}{\partial \mathrm{y}}+\frac{\mathrm{b}}{\bar{\rho}} \frac{\partial \overline{\mathrm{p}}}{\partial \mathrm{y}}-\left[\mathrm{C}_{\mathrm{rp} 1} \mathrm{k}^{2} \sqrt{\mathrm{a}_{\mathrm{n}} \mathrm{a}_{\mathrm{n}}}+\mathrm{C}_{\mathrm{rp} 2} \mathrm{k} \sqrt{\frac{\mathrm{kR} \mathrm{R}_{\mathrm{n}}}{\bar{\rho}}}\right] \mathrm{a}-\frac{\mathrm{R}_{\mathrm{yy}}}{\bar{\rho}^{2}} \frac{\partial \bar{\rho}}{\partial \mathrm{y}} \\
& +\frac{\partial}{\partial \mathrm{k}}\left\{\mathrm{k}^{2} \sqrt{\frac{\mathrm{kR}}{\bar{\rho}}}\left[-\mathrm{C}_{1} \mathrm{a}+\mathrm{C}_{2} \mathrm{k} \frac{\partial \mathrm{a}}{\partial \mathrm{k}}\right]\right\}+\mathrm{C}_{\mathrm{d}} \frac{\partial}{\partial \mathrm{y}} v_{\mathrm{t}} \frac{\partial \mathrm{a}}{\partial \mathrm{y}} .
\end{aligned}
$$

The contributing terms, discussed in the order they appear on the right side, are

1. the advective term relative to the volume-weighted coordinate system.

2. a principal driving term, written as a direct extension of the term in the single-point formulation, on the assumption that spectral b produces spectral $\mathbf{a}$ at the same wave number. From direct numerical simulation calculations, there is reason to believe (Sandoval, 1995) that there are modifications to this term that arise from inclusion of appropriate modeling of the closure for a single-point term like 


$$
\overline{\left(\frac{1}{\rho}\right)^{\prime} \frac{\partial p^{\prime}}{\partial x_{i}}} .
$$

The effect can be described by using some appropriate fraction of $b(k)$ in this source term; we have not, however, included this modification in our present investigations. The assumption that $b(k)$ produces $a_{i}(k)$ at the same $k$ is somewhat justified by the fact that Clark and Spitz (1995) apply an approximate Rapid Distortion Theory to their model for the case of homogeneity and find that: $\frac{\partial a(k)}{\partial t}=b(k) \frac{\partial \bar{p}}{\partial y}$. Sandoval (1995) has also given reasons to believe that this is true from his DNS calculations of homogeneous variable density turbulence. Note that this term contributes a pressure gradient to the equation $\tilde{\mathbf{u}}=\mathbf{a}(\mathrm{y}, \mathrm{t})$, which serves to determine the value of the pressure gradient at every time through the transient dynamics.

3. the representation of drag between the two fluids. The $C_{\mathrm{rp} 1}$ term represents the form drag, with $\mathrm{C}_{\mathrm{rp} 1}$ being directly related to the drag coefficient that Youngs (1992b) required to be an order of magnitude greater than the classical value for an isolated sphere. The length scale that is used by Youngs in the denominator of the drag term is that associated with the thickness of the evolving TMZ as opposed to some fixed sphere radius. We confirm the Youngs finding and attribute the necessity for large magnitude to the effects of convoluted flow paths along the interface between the fluids. The $\mathrm{C}_{\mathrm{rp} 2}$ term represents the viscous drag, in this case resulting from effective turbulence viscosity rather than molecular viscosity. These terms are derived directly as extensions of the usual two-field formulation. There are some possible alternative expressions for these drag terms, which could incorporate relevant effects that are nonlocal in wave number space. For example, we could consider replacing

$$
-C_{\mathrm{pl} l} k^{2} \sqrt{a_{n} a_{n}}
$$


by

$$
-C_{\mathrm{rpl}} \int_{0}^{k_{1}} k \sqrt{a_{n} a_{n}} d k .
$$

In addition, these terms could depend on a nontrivial fashion on dimensionless functions of dimensionless $b(y, t)$. This nonlocal form describes contributions to drag at wave number $\mathrm{k}$ from all other wave numbers lying between $\mathrm{k}=0$ and $k=k_{1}$. There is no difficulty with this formulation if we attempt to let $k_{1} \rightarrow \infty$. With the expectation that $a \rightarrow k^{-7 / 3}$ for large $k$ (see form-function discussion below), the integral converges in that limit. Similar comments can be made about possible nonlocal forms for the $\mathrm{C}_{\mathrm{rp} 2}$ term. It is tempting to consider various nonlocal drag expressions because of the heuristic idea that the effects of many wave numbers can combine collectively to transfer momentum at any particular wave number between the fluids. We have not at this stage found any formulation that works better than the simple local form, insofar as comparisons with experimental data are concerned.

4. a second principal drive term. This is called the gradient-flux term because whenever it is roughly balanced by drag the result is an expression for $\bar{\rho} \mathbf{a}$ (the spectral mass flux), proportional to the gradient of $\bar{\rho}$. Term \#2, in contrast, can contribute a counter-gradient flux, notably when reversal of gravity turns the mixing zone into a demixing zone. More generally, the two principal drive terms, $\# 2$ and \#4, interact with the other terms in the a equation in various possible ways. In the start-up phase of unstable mixing, the pressure-gradient term acts wherever $\mathrm{b}$ is seeded at the interface to drive a wave-like part of $\partial \mathrm{a} / \partial \mathrm{t}$. The growing value of a interacts with the pressure gradient in the $R_{n n}$ and $R_{y y}$ equations (see below), which produces a disordered component of Reynolds stress, which in turn yields a source to a disordered component of $\partial \mathrm{a} / \partial \mathrm{t}$. In this mixing stage of the TMZ growth, the two sources reinforce each other. As the TMZ dynamics mature into 
self-similar growth, the large-wave-number components of b may cascade into an inactive range at higher wave numbers, which means that for those wave numbers the pressure-gradient source to $\partial \mathbf{a} / \partial \mathrm{t}$ has changed to essentially complete balance with the drag term. Only the low-wave-number parts of $b$ (along with the densitygradient source) are effective in perpetuating the continuing self-similar growth of a. The spectrum of $\mathrm{b}$ can be considered to possess active and passive parts, i.e., the low and high wave number parts, which is significant for deriving single-point equations from the spectral equations. More details regarding the concept of active and passive parts of the $b(k)$ spectrum are given in Section 7.3 in the discussion of acceleration reversal. If $\mathbf{g}$ suddenly vanishes, then only the \#4 source term remains to continue widening the TMZ; however, its effectiveness for this purpose is significantly curtailed by the drag (\#3) and cascade (\#5) terms. When $\mathbf{g}$ is completely reversed, we have an adjustment period in which the \#2 source term works in concert with drag (\#3), across all parts (previously active and passive) of the $b$ spectrum, to accomplish the reversal of $a$. This represents a spectral nonequilibrium process. Only when a has changed sign do these terms again oppose each other, and the passive part of the b spectrum returns to dormancy. It is interesting to confirm in calculations of these processes that the passive part of the $b$ spectrum indeed has very little effect on the overall spectrally integrated dynamics, as demonstrated by comparison of two calculations, one that retains the passive part and the other that discards it. The only significant difference between the two calculations lies in the value of $b$; everything else is essentially the same.

5. the cascade terms. These are both conservative in k-space. They are based on the model proposed by Leith (1967) for local cascade, with a wave-like part (the $C_{1}$ term) and a diffusive part (the $C_{2}$ term); see Besnard et al. (1990) for an extensive discussion. With $C_{1}>0$ the wave-like cascade is direct (i.e., to higher wave numbers). Of necessity, $\mathrm{C}_{2}>0$, which results in both direct and inverse 
contributions to cascade. Several nonlocal forms of cascade representation have been considered. These nonlocal forms attempt to represent the triad interactions associated with triple-correlation terms in the formal derivation for constant-density turbulence as integrals over k-space [e.g., EDQNM (Orsag, 1970) or DIA (Kraichnan, 1964) derivations]. Integral formulations for the variable density case are not yet rigorously developed, but one way to accomplish some degree of nonlocality would come (as in the drag term \#3) from replacement of the local cascade rates by nonlocal integral cascade rates; for example,

becomes

$$
\mathrm{k} \sqrt{\frac{\mathrm{kR}_{\mathrm{nn}}(\mathrm{k})}{\bar{\rho}}}
$$

$$
\sqrt{\int_{0}^{k_{1}} k^{2} \frac{R_{n n}(k)}{\bar{\rho}} d k} .
$$

Both forms are essentially equivalent in the inertial range. It should be noted that, because of modeling problems, $R_{n n}(k)$ is not a nonnegative definite function; hence, the simpler local form (5.3.22) will fail for circumstances of $R_{n n}(k)<0$. Clark and Spitz (1995) chose form (5.3.23) on this basis and also on the observation that the so-called "catastrophe time" (Leisure, 1990) for constantdensity isotropic turbulence is predicted with good agreement to EDQNM. However, for most circumstances, we have seen little difference between the results using either cascade rate time-scale, and we have not encountered negative values of $\mathrm{R}_{\mathrm{nn}}(\mathrm{k})$; thus, we have opted for the simpler form (5.3.22). Indeed, it appears, as stated above, that the modeling constraints (conservation, tensor form, dimensionality, etc.) used for our cascade (and other) terms enable the simplest forms (like term \#5) to capture a substantial portion of the physical processes that we wish to represent.

6. spatial diffusion. This term is of the same form as that used by many previous authors. In similar fashion parts of the Boussinesq approximation (Hinze, 1987) 
have been used to represent the diffusion flux of momentum, and the extension of this concept to other turbulence quantities has been made by Daly and Harlow (1970). Two comments are required pertaining to the possible choices for expressions for the eddy viscosity, $v_{t}$ : first, it is well known that the eddy viscosity, $v_{t}$, should be modeled nonlocally; and second, the processes associated with an eddy viscosity are anisotropic. Nevertheless, until further investigation, for simplicity we chose an isotropic form which reduces to the commonly used single point form

$$
v_{\mathrm{t}}=0.09 \mathrm{~S} \sqrt{\mathrm{K}},
$$

in which $\mathrm{S}$ is the mean turbulent scale and $\mathrm{K}$ is the total turbulence energy per unit mass. To accomplish this correspondence requires the identification of a generic form function for $R_{n n}$ and the evaluation of an appropriate moment integral, as discussed below. An appropriate $C_{d}$ must be chosen.

Next, for $b(y, k, t)$ we write

$$
\begin{aligned}
\frac{\partial b}{\partial t} & =\left(\frac{2 \bar{\rho}-\rho_{1}-\rho_{2}}{\rho_{1} \rho_{2}}\right) \frac{\partial \bar{\rho} a}{\partial y}-C_{\mathrm{fb}}\left[\bar{v}^{2} \frac{\partial}{\partial y}\left(\frac{\bar{\rho}}{\bar{v}}\right)\right] \frac{\partial \mathrm{ka}}{\partial \mathrm{k}} \\
& +\frac{\partial}{\partial \mathrm{k}}\left\{\mathrm{k}^{2} \sqrt{\frac{\mathrm{kR} R_{\mathrm{nn}}}{\bar{\rho}}}\left[-C_{1} \mathrm{~b}+\mathrm{C}_{2} \mathrm{k} \frac{\partial \mathrm{b}}{\partial \mathrm{k}}\right]\right\}+C_{\mathrm{d}} \frac{\partial}{\partial \mathrm{y}} v_{\mathrm{t}} \frac{\partial \mathrm{b}}{\partial \mathrm{y}}-C_{\mathrm{db}} \mathrm{k}^{2} \mathrm{Db} .
\end{aligned}
$$

The contributing terms on the right side are

1. the kinematical source term, derived in Appendix B for single-point transport of $b$. Because this term describes the effects of fluid mass transfer, there is no requirement for an advective term in the $b$ equation. This term maintains the value of $b$ at nearly its configurational value, $\alpha_{1} \alpha_{2}\left(\rho_{1}-\rho_{2}\right)^{2 / \rho_{1}} \rho_{2}$.

2. the transport of $b$ through wave-number space, as induced by the presence of inhomogeneity in the mixture of fluids. This term was formally derived by Clark (personal communication, 1994) as a next-higher-order contribution to the Taylor expansion of points, $\mathbf{x}_{1}$ and $\mathbf{x}_{2}$ about the central point, $\mathbf{x}$, for variable density 
inhomogeneous flows. It resembles the $\mathrm{C}_{\mathrm{f}}$ terms previously proposed by Besnard, et al. (1990) in the BHRZ model to describe the mean-flow-shear-induced distortions of turbulence spectra in a constant-density fluid. In the latter case, the term contributes to the vortex-pairing process that occurs in a free shear layer. For this report, it contributes to the bubble-doubling process that is known to occur in the self-similar stages of TMZ growth at small wave numbers and to an alteration of cascade that occurs through "eddy distortion" at high wave numbers. Its presence is very important for the achievement of agreement with experiments. A higherorder degree of nonlocality (e.g., an integral expression) may be appropriate, but at this stage there is no proof of the necessity for this complication.

A heuristic derivation of this term to describe the transport of $b$ through wavenumber space suggests that it must couple the inhomogeneity as described by $\nabla \bar{\rho}$, with the presence of interpenetration, described by a. [Direct coupling to $\nabla \overline{\mathrm{p}}$ seems implausible, as this contributes to the creation of interpenetration via differential accelerations between the different fluids, and is not a measure of the current level of inhomogeneity.] Thus we have

$$
\left(\frac{\partial \bar{p}}{\partial y}\right)\left(\frac{\partial a}{\partial k}\right)
$$

as a basis formulation for this term. Dimensional arguments and the necessity for conservation in $\mathrm{k}$-space suggests a form proportional to

$$
\frac{1}{\bar{\rho}} \frac{\partial \bar{\rho}}{\partial \mathrm{y}} \frac{\partial \mathrm{ka}}{\partial \mathrm{k}},
$$

which is very close to term \#2 of Eq. (5.3.25). Term \#2 of Eq. (5.3.25) and Eq. (5.3.26) become identical for small differences in density.

3. the cascade terms. These have the same forms as the cascade terms for $\mathbf{a}$, and the same comments apply here. It should be noted that cascade rates proportional to $\mathrm{k}^{2} \sqrt{\mathrm{ka} \mathrm{n}_{\mathrm{n}}}$ could be proposed on dimensional grounds, although their physical 
significance is not obvious and their inclusion in calculations produces very minor effects, neither helpful nor harmful in matching results with existing experimental data. Similar to the discussion of (\#5) for the a equation, this expression is not a nonnegative definite function, and a similar argument is used for our choice of cascade rates.

4. the spatial diffusion term. This term has been added to correct a small numerical problem at the center of the TMZ. The term has virtually no effect on any of the other variables and only serves to correct for an anomalous depletion of spectrally integrated $b$ at $y=0$.

5. the decay term resulting from molecular diffusion between species, for which the kinematic diffusion coefficient is $\mathrm{D}$. In the studies described here, $\mathrm{D} \rightarrow 0$.

Next, for $R_{n n}(y, k, t)$ and $R_{y y}(y, k, t)$ we write

$$
\begin{aligned}
\frac{\partial R_{n n}}{\partial t}= & -\frac{\partial R_{n n} \tilde{u}}{\partial y}+\int_{-\infty}^{+\infty}\left(2 a\left(y, y^{\prime}, k\right) \frac{\partial p\left(y^{\prime}\right)}{\partial y^{\prime}}\right)\left(\frac{\exp \left(-2 k\left|y^{\prime}-y\right|\right)}{\int_{-\infty}^{+\infty} \exp \left(-2 k\left|y^{\prime \prime}-y\right|\right) d y^{\prime \prime}}\right) d y^{\prime}-2 R_{y y} \frac{\partial \tilde{u}}{\partial y} \\
& +C_{d} \frac{\partial}{\partial y} v_{t} \frac{\partial R_{n n}}{\partial y}+\frac{\partial}{\partial k}\left\{k^{2} \sqrt{\frac{k R_{n n}}{\bar{\rho}}}\left[-C_{1} R_{n n}+C_{2} k \frac{\partial R_{n n}}{\partial k}\right]\right\},
\end{aligned}
$$

and

$$
\begin{aligned}
\frac{\partial R_{y y}}{\partial t}= & -\frac{\partial R_{y y} \tilde{u}}{\partial y}+\int_{-\infty}^{+\infty}\left(2 a\left(y, y^{\prime}, k\right) \frac{\partial \bar{p}\left(y^{\prime}\right)}{\partial y^{\prime}}\right)\left(\frac{\exp \left(-2 k\left|y^{\prime}-y\right|\right)}{\int_{-\infty}^{+\infty} \exp \left(-2 k\left|y^{\prime \prime}-y\right|\right) d y^{\prime \prime}}\right) d y^{\prime}-2 R_{y y} \frac{\partial \tilde{u}}{\partial y} \\
& +C_{d} \frac{\partial}{\partial y} v_{t} \frac{\partial R_{y y}}{\partial y}+\frac{\partial}{\partial k}\left\{k^{2} \sqrt{\frac{k R_{n n}}{\bar{\rho}}}\left[-C_{1} R_{y y}+C_{2} k \frac{\partial R_{y y}}{\partial k}\right]\right\} \\
& +C_{m} \int_{0}^{k} \sqrt{\frac{k R_{n n}}{\bar{\rho}}} d k\left(\frac{1}{3} R_{n n}-R_{y y}\right) .
\end{aligned}
$$


The contributing terms on the right side are

1. the advective term. In contrast to the a equation, in which the conservation of mass equation is used to remove $\bar{\rho}$ from

$$
\frac{\partial \bar{\rho} \mathbf{a}}{\partial t}+\frac{\partial \bar{\rho} a \tilde{u}_{n}}{\partial x_{n}}
$$

these similar terms in the equations for $R_{i j}$ retain the $\bar{\rho}$ that is intrinsically present in the generalized expression for the Reynolds stress.

2. a principal driving term, which couples a with the pressure gradient. This process is intrinsically nonlocal in physical space, with effects that reach progressively further away for wave numbers approaching zero (i.e., for large scales). The basis for this nonlocality lies in the propagation of pressure waves. In linear KelvinHelmholtz or Rayleigh-Taylor stability analysis the effects are manifested in a velocity potential that varies as $\exp [-\mathrm{k}|\mathrm{y}|]$, where $\mid \mathrm{yl}$ is the distance from the center of the instability layer. Thus the spreading of $R_{i j}$, which varies as the square of velocity fluctuations, is represented by the factor $\exp [-2 \mathrm{klyl}]$, as shown in the term. Otherwise the coupling of a with the pressure gradient is the same as the singlepoint coupling. The basis and consequences of this nonlocality spreading in physical space are discussed further in Section 6.2.

3. another principal driving term, coupling $R_{y y}$ to gradients of $\tilde{u}$. This is a standard term (Besnard, et al. 1990) that is well known especially for constant-density turbulent flows, but which is equally relevant here (although of relatively minor importance for the TMZ studies).

4. the spatial diffusion term. As in the a and b equations, the form to be used can be local or any of several nonlocal variants. As for the a and b equations we use

$$
v_{\mathrm{t}}=\int_{0}^{\infty} \sqrt{\frac{\mathrm{kR}_{\mathrm{nn}}}{\bar{\rho}}} \frac{\mathrm{dk}}{\mathrm{k}^{2}} .
$$


5. the cascade terms. The same formulation and comments apply here as for the cascade of $\mathbf{a}$.

6. the return-to-isotropy term, which appears only in the equation for $R_{y y}$. The return rate can be written either in local form or, as shown, in the nonlocal form

$$
C_{\mathrm{m}} \int_{0}^{\mathrm{k}} \sqrt{\frac{\mathrm{kR}}{\bar{\rho}}} \mathrm{dk} .
$$

Another nonlocal form proposed by Herring (personal communication, 1994) addresses the issues discussed in term \#5 of the a equation and is written as

$$
C_{m} \sqrt{\int_{0}^{k}\left(k^{\prime}\right)^{2} \frac{R_{n n}}{\bar{p}} d k^{\prime}} .
$$

We have tried both forms for the return-to-isotropy rate and have found negligible difference.

Boundary conditions must be considered whenever a finite-difference calculation is performed for limited domains in $\mathrm{k}$ and $\mathrm{y}$. In the $\mathrm{y}$ direction the domain simply extends well into the pure material on either side of the TMZ, where everything is at rest. In k-space we assume that the resolved part of the calculation is bounded by exponential behaviors for both the large and small wave numbers beyond the resolved region. This prescription allows the variable to flux through the boundaries. We also experiment with an exception to this procedure, in which the flux of $\mathrm{b}$ at large wave numbers was set equal to zero, corresponding to $\mathrm{D}=0$. As illustrated and discussed in the chapter on numerical results, Chapter 7 , the effect on $b$ is large; but the difference is almost entirely due to the retention or discarding of the passive parts of the $b$ spectrum, so that the effects on all the other quantities is negligible.

Values for the $\mathrm{C}$ coefficients in these equations have been chosen to be the same as those used by previous investigators for constant-density or single-point studies. For the drag coefficients in the a equation, we use values that are greatly enhanced (Youngs, 1992b) over those for isolated spheres, as discussed earlier in this section. These are

$$
\mathrm{C}_{\mathrm{rpl}}=5.0
$$


and

$$
\mathrm{C}_{\mathrm{rp} 2}=6.0 \text {. }
$$

For the local cascade coefficients, we use the values discussed by Clark (1992), namely,

and

$$
\mathrm{C}_{1}=0.1212 \text {, }
$$

$$
\mathrm{C}_{2}=0.0606 \text {. }
$$

Spatial diffusion is modulated by the coefficient,

while

$$
\mathrm{C}_{\mathrm{d}}=0.03
$$

and

$$
\begin{aligned}
& \mathrm{C}_{\mathrm{db}}=0.0, \\
& \mathrm{C}_{\mathrm{m}}=1.0,
\end{aligned}
$$

$$
\mathrm{C}_{\mathrm{fb}}=0.5 \text {. }
$$

Because $\mathrm{C}_{\mathrm{fb}}$ is the one coefficient that is not determined in previous investigations, we varied its value to determine the effect and observed that it is noticeable but not profound, as described below. Clark (1992) discusses a value of $C_{m}$ for constant density turbulence that is smaller than the value used here. We have tested the model for values of $C_{m}$ between that suggested by $\mathrm{Clark}\left(\mathrm{C}_{\mathrm{m}}=0.17\right)$ and the value we use here and found negligible differences in the results. We suspect that this is due to the fact that our inhomogeneous flow is strongly dominated by the sources due to mixing that renders the return to isotropy a second-order effect.

For reference, we also give the full tensor forms of our base-model equations. In the reference frame with $\overline{\mathbf{u}} \equiv 0$,

$$
\begin{aligned}
\frac{\partial a_{i}}{\partial t}= & -\tilde{u}_{n} \frac{\partial a_{i}}{\partial x_{n}}+\frac{b}{\bar{\rho}} \frac{\partial \bar{p}}{\partial x_{i}}-\left[C_{t p l} k^{2} \sqrt{a_{n} a_{n}}+C_{t p 2} k_{\sqrt{\frac{k R_{n n}}{\bar{\rho}}}}\right] a_{i}-\frac{R_{i n}}{\bar{\rho}^{2}} \frac{\partial \bar{\rho}}{\partial x_{n}} \\
& +\frac{\partial}{\partial k}\left\{k^{2} \sqrt{\frac{k R_{n n}}{\bar{\rho}}}\left[-C_{1} a_{i}+C_{2} k \frac{\partial a_{i}}{\partial k}\right]\right\}+\frac{C_{d}}{\bar{\rho}} \frac{\partial}{\partial x_{n}} v_{t} \bar{\rho} \frac{\partial a_{i}}{\partial x_{n}} \\
\frac{\partial b}{\partial t}= & \left(\frac{2 \bar{\rho}-\rho_{1}-\rho_{2}}{\rho_{1} \rho_{2}}\right) \frac{\partial \bar{\rho} a_{n}}{\partial x_{n}}-C_{f b}\left[\bar{v}^{2} \frac{\partial}{\partial x_{n}}\left(\frac{\bar{\rho}}{\bar{v}}\right)\right] \frac{\partial k a_{n}}{\partial k}
\end{aligned}
$$




$$
+\frac{\partial}{\partial k}\left\{k^{2} \sqrt{\frac{k R_{n n}}{\bar{\rho}}}\left[-C_{1} b+C_{2} k \frac{\partial b}{\partial k}\right]\right\}+C_{d} \frac{\partial}{\partial y} v_{t} \frac{\partial b}{\partial y},
$$

and

$$
\begin{aligned}
& \frac{\partial R_{i j}}{\partial t}=-\frac{\partial R_{i j} \tilde{u}_{n}}{\partial x_{n}}+\int_{-\infty}^{+\infty}\left[\left(a_{i}\left(y^{\prime}\right) \frac{\partial \bar{p}\left(y^{\prime}\right)}{\partial x_{j}}\right)+\left(a_{j}\left(y^{\prime}\right) \frac{\partial \bar{p}\left(y^{\prime}\right)}{\partial x_{i}}\right)\right]\left(\frac{\exp \left(-2 k\left|y^{\prime}-y\right|\right)}{\int_{-\infty}^{+\infty} \exp \left(-2 k\left|y^{\prime \prime}-y\right|\right) d y^{\prime \prime}}\right) d y^{\prime} \\
& -R_{i n} \frac{\partial \tilde{u}_{j}}{\partial x_{n}}-R_{j n} \frac{\partial \tilde{u}_{i}}{\partial x_{n}}+\frac{\partial}{\partial k}\left\{k^{2} \sqrt{\frac{k R_{n n}}{\bar{\rho}}}\left[-C_{1} R_{i j}+C_{2} k \frac{\partial R_{i j}}{\partial k}\right]\right\} \\
& +C_{m} \int_{0}^{k} \sqrt{\frac{k R_{n n}}{\bar{\rho}}} d k\left(\frac{1}{3} R_{n n}-R_{i j}\right)+C_{d} \frac{\partial}{\partial x_{n}} v_{t} \frac{\partial R_{i j}}{\partial x_{n}} .
\end{aligned}
$$




\section{Behavior of the Model Terms in both Physical Space and Spectral Space}

\subsection{Introduction}

In this chapter we present two separate discussions: (1) a detailed analysis of the derivation of the nonlocal pressure source term in the Reynolds stress transport equation (as described in Section 5.3) due to the effects of incompressibility and (2) an investigation of how this nonlocal formulation alters the spectral behavior of our model and how the additional complexities of inhomogeneity and variable density tend to alter the spectra as compared to the case of constant density homogeneous turbulence.

In Section 6.2 we demonstrate the strategy we use in deriving a nonlocal source term for the Reynolds stress tensor transport equation. We appeal to the linear analysis of the Rayleigh-Taylor instability as guidance in deriving a function that will spread the effects of the source term past the edges of the TMZ. The unmodeled source term is made up of a density-velocity correlation $\left(a_{i}\right)$ coupled to the mean pressure gradient.

In Section 6.3 we use schematic drawings to explain the effects of this nonlocal spreading function, $Q\left(y, y^{\prime}, k\right)$, on the spectral behavior of the model. In Section 6.3 we also discuss the various other alterations of the spectrum due to terms in this model. We use the case of constant density homogeneous turbulence as a reference to demonstrate the spectral alterations.

\subsection{Nonlocal processes in physical space}

Integral formulations in physical space enable the characterization of instantaneous pressure-wave propagation of fluctuations from one point in physical space to another. Thus, for example, the existence of mean-flow shear can contribute to the nonlocal creation of turbulence at localities lying outside the shear layer. Likewise a TMZ between two fluids, 
subjected to a mean-field pressure gradient, can manifest the effects of differential acceleration beyond the borders of the mixing zone.

Classical Rayleigh-Taylor analysis for the linear instability of an interface introduces a velocity potential that varies as $\exp (-\mathrm{k}|\mathrm{y}|)$, in which $\mid \mathrm{yl}$ is the distance from the interface (Chandrasekhar, 1961). If we assume that the amplitude of the perturbation on the interface is approximately half the width of the TMZ, then for a TMZ with width $\mathrm{W}$ and for wave numbers such that $\mathrm{kW}>1.0$, the linear theory does not apply. For parts of the turbulence spectrum with $\mathrm{kW} \ll 1.0$, however, the linear theory is relevant and shows that fluctuating components of velocity extend appreciably beyond the boundaries of the TMZ. This "creation-at-a-distance" of velocity fluctuations translates into a nonlocal source for $\mathrm{R}_{\mathrm{ij}}$. It does not, however, indicate nonlocal sources for $\mathrm{a}_{\mathrm{i}}$ or $\mathrm{b}$, which are associated with the transport of fluid rather than pressure effects. We don't use, however, nonlocal sources for $a_{i}$ and $b$. For $b$, there are no pressure terms to provide this effect; for $a_{i}$, preliminary simulations of Sandoval (1995) indicate fluctuating pressure correlations act to modify the $\mathrm{b} \frac{\partial \bar{p}}{\partial y}$, which is already included as a local term.

Guided by the results of linear analysis, we have modified the source terms for $R_{n n}$ and $\mathrm{R}_{\mathrm{yy}}$ in the following manner. The local form,

is rewritten as

$$
\frac{\partial R_{n n}}{\partial t}=2 a \frac{\partial \bar{p}}{\partial y},
$$

$$
\frac{\partial R_{\mathrm{nn}}}{\partial \mathrm{t}}=\int_{-\infty}^{+\infty}\left[2 \mathrm{a} \frac{\partial \bar{p}}{\partial \mathrm{y}}\right] \mathrm{Q}\left(\mathrm{y}^{\prime}, \mathrm{y}\right) \mathrm{d} \mathrm{y}^{\prime},
$$

and similarly for $\mathrm{R}_{\mathrm{yy}}$.

The kernel Q(y'y), "nonlocality function," must satisfy the normalization condition,

$$
\int_{-\infty}^{+\infty} Q\left(y^{\prime}, y\right) d y^{\prime}=1
$$


For our problem we also expect it to depend only on $\mathrm{y}^{\prime}-\mathrm{y}$ and to decrease as $\exp [-2 \mathrm{kly}-\mathrm{yl}]$ as the separation between points increases. For large values of $k, Q\left(y^{\prime}, y\right)$ approaches a delta function. For now, we choose

$$
\mathrm{Q}\left(\mathrm{y}^{\prime}, \mathrm{y}\right)=\mathrm{kexp}\left[-2 \mathrm{k}\left|\mathrm{y}^{\prime}-\mathrm{y}\right|\right]
$$

which satisfies the normalization condition of Eq. (6.2.3), and has the desired delta-function behavior as $\mathrm{k} \rightarrow \infty$. It remains to be demonstrated, however, that this "spreading" factor allows the turbulence model to recover the linear Rayleigh-Taylor behavior as $\mathrm{k} \rightarrow 0$.

To give this demonstration, we will assume that the configuration starts at rest with only $b$ present; the lowest order contribution to the evolution of $a$ and $R_{i j}$ (when both are still very small) is described by the following subset of Eqs. (5.3.28) and (5.3.19). Consider the following parts at first without the $Q\left(y^{\prime}, y\right)$ :

and

$$
\frac{\partial R_{y y}}{\partial t}=2 a \frac{\partial \bar{p}}{\partial y}
$$

$$
\frac{\partial \mathrm{a}}{\partial \mathrm{t}}=\frac{\mathrm{b}}{\bar{\rho}} \frac{\partial \overline{\mathrm{p}}}{\partial \mathrm{y}}-\frac{\mathrm{R}_{\mathrm{yy}}}{\overline{\mathrm{\rho}}^{2}} \frac{\partial \bar{\rho}}{\partial \mathrm{y}}
$$

Differentiate the $\mathrm{R}_{\mathrm{yy}}$ equation with respect to $t$, allowing only for the variation of $\mathbf{a}$, and insert $\partial \mathrm{a} / \partial \mathrm{t}$ from the second equation. Then

$$
\frac{\partial^{2} R_{y y}}{\partial t^{2}}=-\frac{2}{\bar{\rho}^{2}} \frac{\partial \bar{p}}{\partial y} \frac{\partial \bar{\rho}}{\partial y} R_{y y}+\frac{2 b}{\bar{\rho}}\left(\frac{\partial \bar{p}}{\partial y}\right)^{2} .
$$

The second term on the right, in the qualitative fashion of the other terms here omitted, is not relevant to this discussion, while the first term on the right contributes either an oscillatory component (if $\left(\frac{\partial \bar{p}}{\partial y}\right)\left(\frac{\partial \bar{p}}{\partial y}\right)>0$ ) or an exponentially growing component (if $\left(\frac{\partial \bar{p}}{\partial y}\right)\left(\frac{\partial \bar{\rho}}{\partial y}\right)<0$ ). This last would appear to be the classic linear Rayleigh-Taylor solution but fails in that regard in one crucial respect. 
To demonstrate this failure and its remediation, we note that, as it is written in local form, the first term distributes the turbulence energy only within the mixing layer itself. In that region for the unstable case, $R_{y y}$ grows as

$$
R_{y y}(t)=R_{y y}(0) \exp \left[t \sqrt{\frac{2}{\bar{\rho}^{2}}\left|\frac{\partial \bar{p}}{\partial y} \frac{\partial \bar{\rho}}{\partial y}\right|}\right] \text {. }
$$

Letting $g \equiv(1 / \bar{\rho})(\partial \bar{p} / \partial y)$ and $\partial \bar{\rho} / \partial y=\Delta \rho / W$, where $\Delta \rho$ is the overall density difference across the mixing layer of width $\mathrm{W}$, we get

$$
R_{y y}(t)=R_{y y}(0) \exp \left[t \sqrt{\frac{2}{W}\left|g \frac{\Delta \rho}{\bar{\rho}}\right|}\right] .
$$

In contrast, for an infinitesimal velocity squared, classic linear Rayleigh-Taylor analysis gives (Harlow and Amsden, 1971)

$$
R_{y y}(t)=R_{y y}(0) \exp \left[2 t \sqrt{k\left|g \frac{\Delta \rho}{2 \bar{\rho}}\right|}\right] .
$$

(Note the factor of 2, to describe growth of velocity-squared.) The essential difference between these two results is the occurrence of $1 / \mathrm{W}$ in the first and $\mathrm{k}$ in the second. In both cases, $R_{y y}(0)$ may depend on $k$, but the turbulence-transport result is essentially independent of $\mathrm{k}$ in the exponent. The reason, of course, is that the creation of $\mathrm{R}_{\mathrm{yy}}$ in the purely local formulation confines the inertial resistance to growth to the mass within the mixing layer itself. In reality, as described above, the mass that must be set in motion extends well beyond the edges, so that $R_{y y}$ can be expected to grow with a much reduced exponent for structures that are large compared to $\mathrm{W}$, i.e., for $\mathrm{kW} \ll 1$. Thus this local formulation preserves the spectral structure of $\mathrm{R}_{\mathrm{yy}}(0)$.

With nonlocality of $Q\left(y^{\prime}, y\right)$, the turbulence-transport results account for the added inertia and give the classical exponential growth. As a practical consequence, the behavior of TMZ growth and appearance is significantly altered; as a conceptual consequence, the 
influence of low wave numbers on self-similarity is quite different from that of the purely local theory. The nonlocal formulation does not preserve the spectral structure of $R_{y y}(0)$.

To demonstrate the remedial effects of including the nonlocal pressure-wave propagation, we return to the equation for $\mathrm{R}_{\mathrm{yy}}$ which is rewritten as follows:

$$
\frac{\partial R_{y y}}{\partial t}=\int_{-\infty}^{+\infty} S\left(y^{\prime}\right) k \exp \left[-2 k\left|y^{\prime}-y\right|\right] d y^{\prime}
$$

where

$$
\mathrm{S}(\mathrm{y}) \equiv 2 \mathrm{a} \frac{\partial \overline{\mathrm{p}}}{\partial \mathrm{y}}
$$

To isolate the essence of our demonstration, it is sufficient to consider, for $y>W / 2$, the approximation (an over estimation by choosing $S(y)$ at $y=0$ )

$$
\begin{aligned}
\frac{\partial R_{y y}}{\partial t} & =\int_{-W / 2}^{W / 2} S(0) k \exp \left[-2 k\left|y^{\prime}-y\right|\right] d y^{\prime} \\
& =S(0) \exp [-2 k y]\left(\frac{\exp (k W)-\exp (-k W)}{2}\right) .
\end{aligned}
$$

Twice the integral from W/2 to $\infty$ thus gives the total amount of $R_{y y}$ created per unit time outside of the TMZ, which, accordingly, is

$$
\frac{\partial}{\partial t} R_{y y}(\text { outside })=S(0)\left[\frac{1-\exp (-2 \mathrm{~kW})}{2 \mathrm{k}}\right] \text {. }
$$

The total amount created per unit time everywhere is (because of the normalization of Q) the same as the local-theory total amount, namely $\mathrm{S}(0) \mathrm{W}$. Thus the nonlocal prediction for total $R_{y y}$ created inside the layer per unit time is

$$
\frac{\partial}{\partial t} R_{y y}(\text { inside })=S(0) W\left[1-\frac{1-\exp (-2 k W)}{2 k W}\right] .
$$

For $\mathrm{kW}<1$, we expand the exponent to get

$$
\frac{\partial}{\partial t} R_{y y}(\text { inside }) \approx S(0) k W^{2} .
$$

Divide this by $\mathrm{W}$ to get, within the layer, 


$$
\frac{\partial \mathrm{R}_{\mathrm{yy}}}{\partial \mathrm{t}}=2 \mathrm{akW} \frac{\partial \overline{\mathrm{p}}}{\partial \mathrm{y}}
$$

With this alteration the exponential growth for $\mathrm{kW}$ small becomes

$$
R_{y y}(t)=R_{y y}(0) \exp \left[t \sqrt{2 k\left|g \frac{\Delta \rho}{\bar{\rho}}\right|}\right],
$$

which now contains the essential dependence on $\mathrm{k}$ seen in the solution to the classic linear Rayleigh-Taylor analysis, Eq. (6.2.10).

It is interesting to contrast our approach to nonlocal pressure-wave effects with the technique employed by Demuren, et al. (1994). They describe a procedure for "local diffusion sources to be distributed over lengths of the order of the integral scale." They implement this technique into a nonspectral transport model and report that it "enabled the well-known free-stream edge singularity problem to be eliminated." To the extent that turbulence self-diffusion receives contributions from the nonlocal triple-correlation terms that arise from pressure-velocity correlations through Greens-function integral solutions, we agree that turbulence diffusion should be distributed nonlocally. This effect is physically visualized in a two-point (spectral) formulation of the theory. To capture the effects of inertial response on the creation of turbulence (and thus the Rayleigh-Taylor linear growth rate), we believe the principal nonlocal effect must be as described by our $Q\left(y^{\prime}, y\right)$ modification to the differential-acceleration term (and to the mean-flow shear coupling).

It is of interest to note the relation between linear Rayleigh-Taylor analysis and the self-similar analysis for the turbulence equations described in Chapter 7. If there is a rich spectrum of modes at $\mathrm{t}=0$, then each will grow exponentially, then saturate, creating an envelope for net growth of the fully nonlinear evolution (see Fig. 6.2.1).

This envelope has the quadratic behavior dictated by the dimensionality of acceleration, g. A heuristic analysis of this process starts with the linear growth equation for amplitude, A, at wave number $\mathrm{k}$, and Atwood number At, namely, 


$$
\frac{\mathrm{d}^{2} \mathrm{~A}}{\mathrm{dt}^{2}}=\mathrm{kg}(\mathrm{At}) \mathrm{A},
$$

and replaces $\mathrm{k}$ by $1 / \mathrm{S}$, where $\mathrm{S}$ is the currently dominant scale at which growth is occurring. With self-similarity, $S$ is a fixed multiple of $A$, that is, $S=\beta A$. Then

and

$$
\frac{d^{2} A}{d t^{2}}=\frac{g(A t)}{\beta},
$$

$$
A=\frac{1}{2 \beta}(A t) g^{2} .
$$

If mode saturation were to occur when $\mathrm{A}$ is approximately $20 \%$ of $\mathrm{S}$, then we get $\mathrm{A}=0.1 * A t^{*} \mathrm{~g}^{*} \mathrm{t}^{2}$, which is consistent with the actually observed self-similar behavior of a TMZ, for which experiments give a coefficient of about 0.12 .

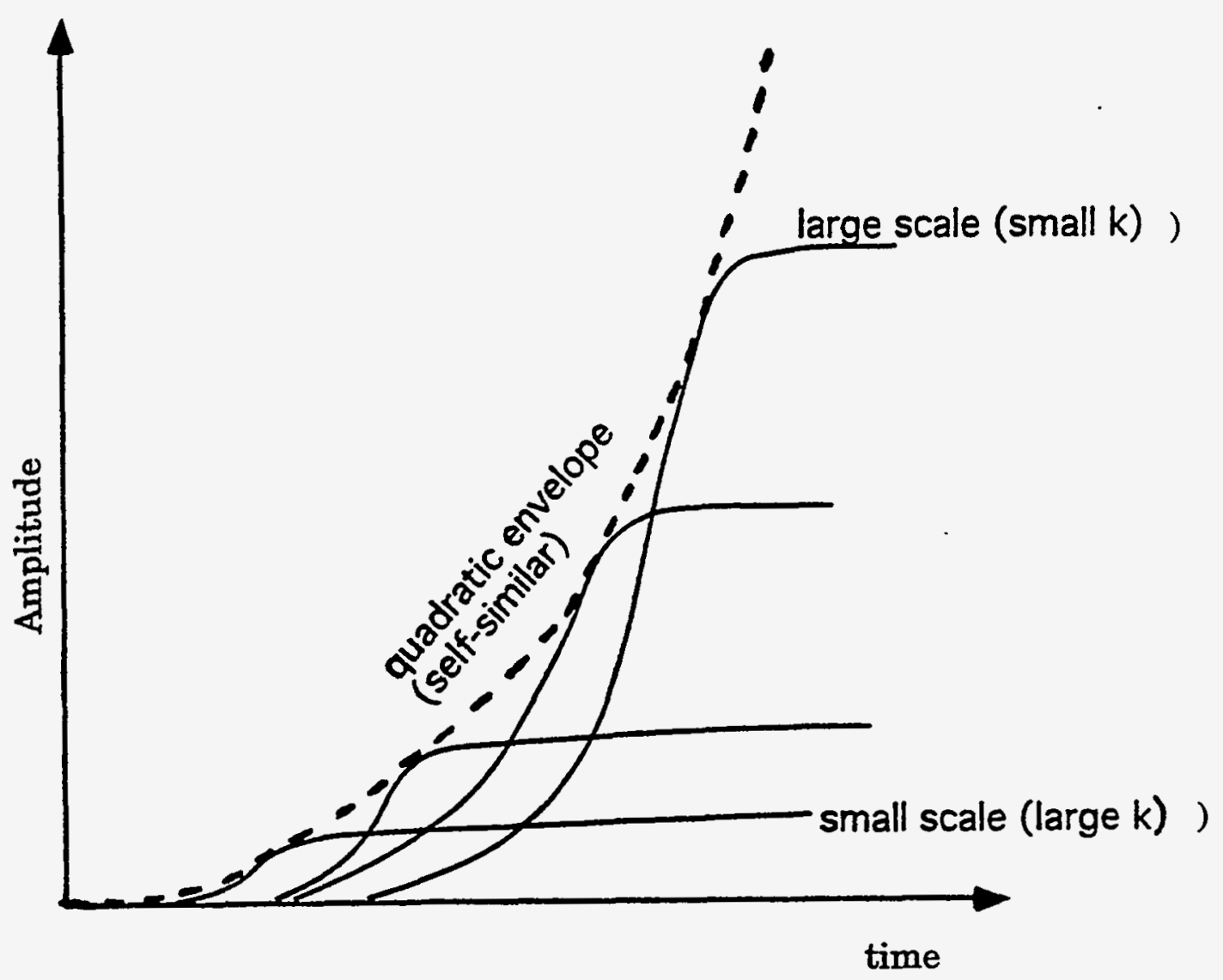

Figure 6.2.1 Schematic to demonstrate the growth and saturation of initial perturbations of the Rayleigh-Taylor instability. 


\subsection{Spectral behavior of the model terms}

As compared to the case of decaying homogeneous constant density turbulence, the added complexity of inhomogeneity and variable density changes the spectral behavior of $R_{i j}(k)$. The additional variables that are a consequence of the variable density, namely $a_{i}(k)$ and $b(k)$, also contribute to altering the spectral shape of $R_{i j}(k)$. In this section we examine the influence that these variables have on the spectra. In order to systematize the discussion, we first address the complexities associated with inhomogeneous turbulence and then discuss the resulting alterations in the spectra by splitting the spectra into two sections: (A) the high wave numbers and (B) the low wave numbers. For each section we identify the terms in the model that have the greatest influence on the behavior of the spectra. For each of the terms, we discuss how they interact with other terms in order to alter the spectrum.

The BHRZ model (1990) shows the existence of self-similarity upon substitution of the self-similar form for the homogeneous isotropic turbulence energy spectrum as suggested by Von Karman and Howarth (1938) into their spectral model. With our variable-density circumstances the relevance of these simple observations is even less apparent. In this section we show the basis for numerous deviations from simple self-similarity in these more general circumstances. In Appendix E, we nevertheless describe a possible set of moment equations based on self-similarity. To proceed with this discussion, however, we will comment on the specific ways in which we can characterize the spectrum associated with the quantities of our model for variable density turbulence.

Here we state the general principal that we follow for this study based on our beliefs about the nature of the attainment and persistence of the structure of spectral self-similarity. The entire evolution of spectral self-similarity is the direct result of a competition of

processes that drive a flow. For the case of the decay of isotropic constant-density homogeneous turbulence, the identification of these competing processes is very simple and straightforward. The energy spectrum, $E(k)$, where $E(k)=E_{11}(k)+E_{22}(k)+E_{33}(k)$, is altered only by the transfer of energy among different scales. This transfer can be 
represented by the local diffusion model (Leith, 1967) which accounts for cascade to higher wave numbers as well as the diffusion to both higher and lower wave numbers. For decaying constant density isotropic turbulence, the transfer of energy through the inertial range of the spectrum is conservative. Besnard et al. (1990) give a discussion pertaining to the relationship between spectral self-similarity and the competition between the cascade and viscous dissipation terms.

For the case of constant density homogeneous anisotropic turbulence with a source, such as an infinite shear layer, the balance between processes must also include the source, which in this case is due to the presence of the $E_{12}(k)$ spectrum coupled to the mean flow gradient. The $E_{12}(k)$ spectrum acts as a source to the $E(k)$ spectrum at the smaller wave numbers. This is possible because the $E_{12}(k)$ spectrum goes as $k^{-7 / 3}$, and the $E(k)$ spectrum goes as $\mathrm{k}^{-5 / 3}$. Thus we have a source, namely $\mathrm{E}_{12}(\mathrm{k})$, continuously pumping energy into the $E(k)$ spectrum at the lower wave numbers, the conservative cascade of energy to the higher wave numbers, and the final decay of energy at the highest wave numbers. As in the isotropic case, once an equilibrium is reached among the different processes, the energy spectrum assumes a self-similar time-independent shape.

For the case of inhomogeneous anisotropic constant density turbulence, such as a free shear layer, additional facets such as the diffusion, advection, and nonlocal sources to energy in physical space significantly complicate this balance of processes. With these additional facets to the flow, the cascade of energy through the inertial range from low to high wave numbers is no longer a conservative process. Account must also be taken for the transfer of energy in physical space. For example, in a given control volume in $(k, y)$-space, the transfers of the various Reynolds stress tensors are still conservative; but the added dimension in the $y$ direction distinguishes this from the homogeneous case. The sources are still predominately at the small wave numbers, and the sink is still at the highest wave numbers. For this case, the inertial range contains sinks and sources due to the inhomogeneity resulting in a nonconservative cascade of energy through the inertial range. 
Another complexity that anisotropy adds is the addition of the remaining six components of the Reynolds stress tensor that do not contribute for the case of isotropic turbulence. This facet of self-similarity is difficult to represent schematically. The coupling of these components with one another and the exchange of energy that occurs between them also complicate the competition of processes.

For flows that contain a time-dependent source, the characteristic time for the different processes that are responsible for the transfer of energy becomes an issue. For example, if a flow has reached a state of equilibrium between all of its competing processes that transfer energy and the driver of the flow undergoes some type of transient, then the rate of return of the flow to a state of equilibrium is governed by a competition between these characteristic times. The processes must compete with one another until an equilibrium is reached and the flow is once again self-similar.

If we now allow the density to vary in the inhomogeneous circumstance, the competition between processes becomes even more involved due to the transfer of energy between the added functions that arise as a consequence of these density fluctuations. Referring to the equations of our model, we see that $b$ drives $a_{j}$ and $a_{j}$ in turn drives $R_{i j}$ which feeds back into $b$ and $a_{i}$. Of course, a transient in the driver of the flow will also result in greater complications.

As an example of the spectral altering effects of inhomogeneity, consider the turbulent viscosity, $v_{t}$, which is present in the spatial diffusion term of each equation of our model. Letting $\varphi$ represent any one of our transported turbulence variables, the turbulent viscosity appears in our model equation like

$$
\frac{\partial \varphi(k, x, t)}{\partial t}=\frac{\partial}{\partial x_{n}} v_{t}(\cdots) \frac{\partial \varphi(k, x, t)}{\partial x_{n}}+\ldots .
$$

The turbulent viscosity can be modeled in either a local (in k-space) version, e.g.,

$$
v_{t}(x, k, t)=\frac{\sqrt{k E(x, k, t)}}{k}
$$


which maintains k-dependence; or any one of various types of nonlocal (in k-space) versions, e.g.,

and,

$$
v_{t}(k, x, t)=\int_{0}^{k} \frac{\sqrt{k^{\prime} E\left(k^{\prime}, x, t\right)}}{\left(k^{\prime}\right)^{2}} d^{\prime}
$$

$$
v_{t}(x, t)=\int_{0}^{\infty} \frac{\sqrt{k E(x, k, t)}}{k^{2}} d k,
$$

the first of which maintains k-dependence and the latter which is independent of $k$. The second of the two nonlocal versions of $v_{t}$ is a consequence of considering the effect of all scales of the turbulence on the random walk process associated with diffusion. This consideration renders the turbulent viscosity independent of $k$. The first version accounts for only the wave numbers smaller than the point in the spectrum. The local version represents only the influence on the diffusive process due to eddies associated with the same wave number. A third version that has been suggested by Heisenberg (1948) which would account for the action of all wave numbers larger than the wave-number location, suggesting that it is the smaller turbulent structures that most influence the diffusive process, is written as

$$
v_{t}(k, x, t)=\int_{k}^{\infty} \frac{\sqrt{k^{\prime} E\left(k^{\prime}, x, t\right)}}{\left(k^{\prime}\right)^{2}} d k^{\prime} .
$$

This type of representation is also dependent on wave number $\mathrm{k}$.

We emphasize the nature of the modeling for the turbulent viscosity, i.e., whether or not it is dependent on the wave number $\mathrm{k}$ (roughly associated with the size of the eddies). For the case where $v_{t}$ is independent of wave number, the effective diffusion of the transported variable will be a flux proportional to the gradient that remains constant throughout the entire spectrum. For this particular case, as shown in Eq. (6.3.1) the diffusion term has no explicit dependence on $k$ so that $\partial \varphi / \partial t$ has the same spectral form as $\varphi$ itself, resulting in no alterations of the spectrum due to the diffusion term. Since the same sink/source is "felt" on the entire spectrum, the diffusion term cannot be responsible for altering the neighboring (in physical space) spectra in any way. Contrarily, if a version of the 
turbulent viscosity is used which renders $v_{t}$ dependent on the wave number $k$, the variation in the diffusion for a given point in physical $\mathrm{y}$-space for different magnitudes of $\mathrm{k}$ will alter the spectral shape of $\varphi$. The variation in the level of diffusion in physical space, $y$, for all the different wave numbers effectively alters the neighboring spectra resulting in different spectral behaviors.

Thus, the two main effects due to the inhomogeneity are (1) lateral spectrum changes as we move in $y$-space, and (2) the "inertial range" is altered even at the same point in $y$ space. That is to say, that not only the adjacent spectra are altered, but also the cascade flux in $\mathrm{k}$-space is no longer constant in the "inertial range."

To illustrate this quantitatively, suppose that the energy tensor, $\mathrm{E}(\mathrm{k})$, behaves like $\mathrm{k}^{-}$ $5 / 3$ through the inertial range. Then the local version of the turbulent viscosity, Eq. (6.3.2), will behave as $\mathrm{k}^{-4 / 3}$ through the inertial range, and since the form of the diffusion terms in this model is that found in Eq. (6.3.1), a quantity that behaves like $\mathrm{k}^{-9 / 3}$ through the inertial range will be diffused to the neighboring locations in physical space.

In view of the above behavior, one may ask the question: does this behavior mean that we have lost the type of self-similarity that has been observed by Besnard et al. (1990) for the case of decaying constant-density homogeneous isotropic turbulence? The answer to this question is probably not. We believe that the spectrum evolves to a self-similar shape. The important concept to recognize here is the method used to detect a self-similar evolution of the spectrum and the fact that $k_{\max }$ (the value of $\mathrm{k}$ at which the spectrum attains an extremum) exhibits spatial dependence.

In the process of determining if a spectrum is evolving self-similarly, two things must be done to a time sequence of spectral plots: (1) the magnitudes of the spectra are rescaled such that the extremum of the spectra coincide, and (2) the positions of the spectra are rescaled in such a way that all spectra attain their extremum at the same wave number. With these two rescalings, the spectra are said to be evolving self-similarly if a time sequence of 
spectra can be made to overlay one another. The degree to which one may say that the spectrum is evolving self-similarly is the degree to which the overlaid plots coincide.

To rescale spectra such that the maxima coincide, the value of $\mathrm{k}_{\max }$ must be known. A feature of this study is the recognition that $\mathrm{k}_{\max }$ is a function of the spatial variable $\mathrm{y}$. With this identification of a spatially dependent $k_{\max }$, we can effectively rescale the spectra so as to test for the self-similar behavior of the spectrum as functions of both $y$ and $t$. This merely amounts to a different rescaling for each position in y-space through the mixing layer. The evolution of the spectrum may converge to an exact self-similar shape but we are discovering the importance of the concept of "almost" self-similar, and the evolution to a generic form of the spectra.

\subsection{A High wave numbers}

We now examine an important characteristic of the $a_{i}(k)$ spectrum at high wave numbers. It is shown that as a consequence of the decay terms in the $a_{i}(k)$ equation, the $a_{i}(k)$ spectrum falls off much more rapidly for high wave numbers than do the spectra for either $b(k)$ or $R_{i j}(k)$. Since $a_{i}(k)$ is the principal source to both $b(k)$ and $R_{i j}(k)$, this behavior of the $a_{i}(k)$ spectrum results in a source term to $b(k)$ and $R_{i j}(k)$ that is effectively localized to the lower wave numbers. This behavior of the source term is closely aligned with the behavior of the source term due to a shear layer in a constant density flow as previously discussed in this section.

The behavior of the high wave-number part of the $a_{i}(k)$ spectrum is dominated by a competition among four terms in the transport equation of $a_{i}(k)$. These four terms are the two source terms, i.e., the $[b(k)] \partial \bar{p} / \partial x_{i}$ term and the $\left(R_{i n}(k) / \bar{\rho}^{2}\right)\left(\partial \bar{\rho} / \partial x_{n}\right)$ term, and the two drag terms, i.e., the $\left(-C_{r p 1} k^{2} \sqrt{a_{n}(k) a_{n}(k)}\right) a_{i}(k)$ term and the $\left(-C_{r p 2} k \sqrt{k R_{n n}(k) / \bar{\rho}}\right) a_{i}(k)$ term. A simple analysis of the competing terms in the transport of the $a_{i}(k)$ equation shows that the high wave-number behavior of $a_{i}(k)$ will either vary as $k^{-11 / 6}$ or $k^{-7 / 3}$, depending on which drag term is dominating. Due to the lack of any drag-like decay terms in the $R_{i j}(k)$ and $b(k)$ equations, the behavior of these quantities is dominated by the condition of nearly 
constant flux in $k$-space, so that they vary closely as $k^{-5 / 3}$ for the high wave numbers (see Fig. 7.2.3(b)). To demonstrate the consistency of these conclusions of $a_{i}(k)$ for high wave numbers, we use the fact that the two source terms in the $a_{i}(k)$ equation vary nearly as $k^{-5 / 3}$ and set up a balance among them and the decay terms. For this examination of the high wave numbers, let us represent the vector component $a_{i}$ as some constant multiplied by a power, $n$, of $\mathrm{k}$, i.e.,

$$
a_{i} \rightarrow a_{0} k^{n}
$$

Likewise, we can closely approximate $R_{i j}$ and $b$ in a similar fashion with

and

$$
\mathrm{R}_{\mathrm{ij}} \rightarrow \mathrm{R}_{0} \mathrm{k}^{-5 / 3}
$$

$$
b \rightarrow b_{0} k^{-5 / 3}
$$

With these expressions, a balance among the source and decay terms on the right side of the $a_{i}(k)$ equation becomes

$$
\left(\mathrm{b}_{0} \frac{\partial \overline{\mathrm{p}}}{\partial \mathrm{y}}+\frac{\mathrm{R}_{0}}{\bar{\rho}^{2}} \frac{\partial \overline{\mathrm{p}}}{\partial \mathrm{y}}\right) \mathrm{k}^{-5 / 3} \approx\left(\mathrm{C}_{\mathrm{rpl}} \mathrm{k}\left|\mathrm{a}_{0} \mathrm{k}^{\mathrm{n}}\right|+\mathrm{C}_{\mathrm{rp} 2} \mathrm{k}^{\frac{\mathrm{kR} \mathrm{R}_{0} \mathrm{k}^{-5 / 3}}{\bar{\rho}}}\right) \mathrm{a}_{0} \mathrm{k}^{\mathrm{n}}
$$

From this expression, if the $\mathrm{C}_{\mathrm{rp} 1}$ term is dominating, the following balance must exist between the exponents of $\mathrm{k}$ :

$$
\frac{-5}{3}=2(\mathrm{n}+1)
$$

from which we see that we get $n=-11 / 6$. This is the expected power of the high wavenumber behavior of $\mathrm{a}(\mathrm{k})$ if the $\mathrm{C}_{\mathrm{rpl}}$ term dominates the decay.

If the $\mathrm{C}_{\mathrm{rp} 2}$ term dominates the high wave-number behavior then the following balance is established:

$$
-\frac{5}{3}=\frac{3}{2}-\frac{5}{6}+\mathrm{n}
$$

from which we see that the power law behavior for the high wave number part of the spectrum for $a_{i}(k)$, becomes $n=-7 / 3$. Numerically we observe this (see Fig. 7.2.2(a)) and see that indeed it is the $\mathrm{C}_{\mathrm{rp} 2}$ term that dominates the decay process. (This dominance is also 
observed in calculations that show considerable insensitivity to the value of $C_{\mathrm{rp} 1}$ through any reasonable variations of its magnitude.)

Now that we have established that for the self-similar regime, the high wave number for $a_{i}(k)$ goes as either $k^{-11 / 6}$ or $k^{-7 / 3}$ it follows that since $b(k)$ and $R_{i j}(k)$ both go as $k^{-5 / 3}$ for high wave numbers, $a_{i}(k)$ is a source that is localized to the low wave numbers for both $b(k)$ and $\mathrm{R}_{\mathrm{ij}}(\mathrm{k})$, and the assumption of cascade dominance for them in the inertial range is confirmed. In this manner, this situation is much like any anisotropic flow. Since the source for both the $b(k)$ and the $R_{i j}(k)$ equation is localized to the low wave numbers, there is a region of nearly constant flux through the inertial range for both $b(k)$ and $R_{i j}(k)$ allowing the spectrum to develop into a $\mathrm{k}^{-5 / 3}$ spectral behavior. The flux is constant in this region for $\mathrm{b}(\mathrm{k})$.

We should note however, that if $v_{\mathrm{t}}$ varies with $\mathrm{k}$, then there is a possible modification to the inertial spectra for $b(k)$ and $R_{i j}(k)$ arising from variations with $k$ of the $y$-direction diffusive flux (refer to the earlier discussion in this section).

Conversely, for high wave numbers, $b(k)$ creates $a_{i}(k)$ in $a k^{-5 / 3}$ fashion across the inertial range. However, as stated above, the drag terms of $a_{i}(k)$ dominate in this region, which drives the spectrum to a behavior more closely dominated by these drag terms.

\subsection{B Low wave numbers}

We have seen the effective localization to small wave numbers of the source terms for $R_{i j}(k)$ and $b(k)$ due to the decay terms in the $a_{i}(k)$ equation. We now examine the low wavenumber behavior of the sources for this model. We first examine the source term of the $R_{\mathrm{ij}}(\mathrm{k})$ equation and discuss the modifications resulting from the nonlocal extension to this term, which we have implemented into the model. We then describe the low wave number behavior of the source terms of the $a_{i}$ and $b$ equations and other terms that play significant roles in the spectral modifications at low wave numbers.

The main source term of the $R_{i j}(k)$ equation is composed of the net mass fluxing velocity, $a_{i}$, coupled to the mean pressure gradient, i.e., $\left[a_{i}(k)\right] \partial \bar{p} / \partial x_{j}+\left[a_{j}(k)\right] \partial \bar{p} / \partial x_{i}$. This 
term was examined in Section 6.2. Past authors of single-point and two-point spectral models have treated this source term due to the mean pressure gradient as a purely local term (BHR, 1987; BHRZ, 1990; Andronov, et al. 1982). That is to say, the production of $\mathrm{R}_{\mathrm{ij}}$ at a point $\mathbf{x}$ is influenced by the mean pressure gradient only at that same position, $\mathbf{x}$. Due to incompressibility, we know that acoustic signals transfer the effects of the mean pressure changes instantaneously throughout the entire fluid, a global mechanism. In our model, in addition to the spectral formulation, we also formulate this source term for $R_{i j}(k)$ nonlocally in physical space (see Section 6.2). The formulation of this nonlocal source term is easier to specify for a spectral model as opposed to a single-point model. The nonlocal spectral formulation is chosen to agree with the known spectral behavior for Rayleigh-Taylor analysis at low wave numbers. The additional information regarding length scales resulting from a spectral formulation enhances our ability to capture the global effects due to incompressibility.

However, as compared to a local source term for $\mathrm{R}_{\mathrm{ij}}(\mathrm{k})$, a nonlocal source term does change the spectral behavior of the model. The far reaching effects of the nonlocal term, i.e., the $\left|y^{\prime}-y\right|$ factor in the exponential of the $Q$ function as discussed in Section 6.2, directly modify the $R_{i j}(k)$ spectrum and indirectly modify the $a_{i}(k)$ and $b(k)$ spectra through the coupling to $R_{n n}(k)$ in the local cascade rate and through the coupling to $R_{y y}(k)$ in the density gradient source term to $a_{i}(k)$.

Here we examine the modifications to the spectral behavior of the model due to the nonlocal source for $R_{i j}(k)$. We proceed by examining the two-dimensional $(y, k)$ shape of a purely local source for $R_{i j}(k)$ within the TMZ. Since $a_{i}(k)$ is zero outside of the TMZ (no net mass flux), the local source, $S_{i j}^{L}(y, k, t)$, for $R_{i j}(k)$ is likewise zero outside of the TMZ. We show the mechanism by which the local source undergoes a spreading over physical space due to the nonlocal modifications to the local source $S_{\mathrm{ij}}^{\mathrm{L}}(\mathrm{y}, \mathrm{k}, \mathrm{t})$, where

$$
S_{i j}^{L}(y, k, t)=a_{i}(y, k, t) \frac{\partial \bar{p}(y, t)}{\partial x_{j}}+a_{j}(y, k, t) \frac{\partial \bar{p}(y, t)}{\partial x_{i}} .
$$


Figure 6.3.1 gives a qualitative idea of the nature of the effects of a local source term on $\partial R_{i j}(k) / \partial t$. The wave number $k 1$ is the wave number at which $a_{i}(k)$ attains its extremum. The TMZ is shown as the horizontal strip. The drawings across the horizontal TMZ represent, qualitatively, the relative magnitudes of the local source term in the $R_{i j}(k)$ equation. The purpose of these sketches is to depict the influence of the spectral shape of $a_{j}(k)$ on the source term of $\partial R_{i j}(k) / \partial t$. At $k=k 1$, the contribution to $\partial R_{i j}(k) / \partial t$ of the

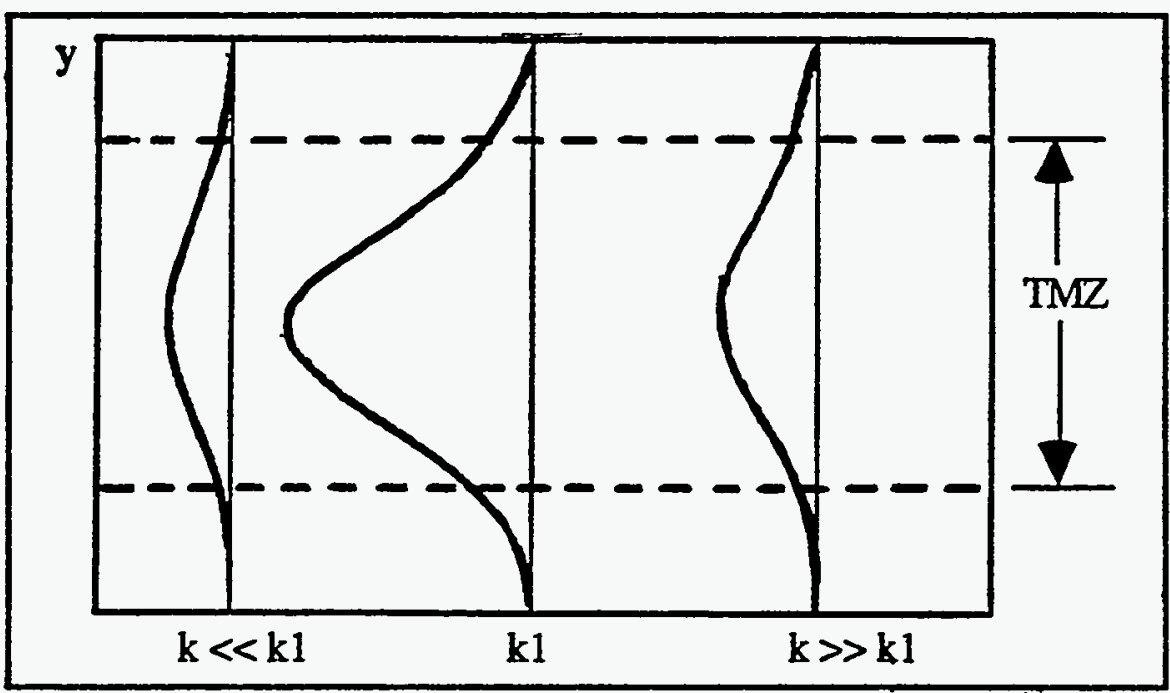

Figure 6.3.1. Schematic of the local source term across the TMZ as a function of $y$ and $k$. The maximum source corresponds to the wave number $k 1$ where the spectrum of $a(k)$ attains an extremum.

source term has a maximum while it tapers off to zero for both the low and high wave numbers of the spectrum. Notice also that the local source term goes to zero at both edges of the TMZ.

We now describe the smearing (across physical space) effect that the incorporation of the nonlocality has on the otherwise local source, $S_{i j}^{L}(y, k, t)$. As shown in Section 6.2, the form of the nonlocal source term is derived from considerations of Rayleigh-Taylor theory. The nonlocal source is written as

$$
\int_{-\infty}^{+\infty} S_{i j}^{L}\left(y^{\prime}, k, t\right) Q\left(y^{\prime}, y, k, t\right) d y^{\prime} .
$$


We see that the source to $R_{i j}(k)$ at point $y$ receives contributions from $S_{i j}^{L}$, the local term, integrated over all space; and it is the $Q$ function that couples the global effects of $S_{i j}^{L}$ back to the point of production. Since the $\mathrm{Q}$ function has the form

$$
\mathrm{Q}\left(\mathrm{y}^{\prime}, \mathrm{y}, \mathrm{k}, \mathrm{t}\right)=\mathrm{Q}_{0}(\mathrm{k}, \mathrm{t}) \exp \left(-\mathrm{k}\left|\mathrm{y}^{\prime}-\mathrm{y}\right|\right),
$$

it will be responsible for altering the shape of the spectrum due to the $\mathrm{k}$ in the exponential. (The $\mathrm{Q}_{0}(\mathrm{k}, \mathrm{t})$ is a normalizing function which guarantees a global conservation of the source term over physical space).

If we first examine only the form of $Q\left(y^{\prime}, y, k, t\right)$ uncoupled from the source term, we see that the $\mathrm{k}$ in the exponential gives very different forms for large and small values of $\mathrm{k}$. Figure 6.3.2 shows the different forms the $\mathrm{Q}$ function will take for different values of $\mathrm{k}$. The structural feature that is important to notice in Fig. 6.3.2 is the narrow base width for large values of $\mathrm{k}$ as compared to the spread out structures for the smaller values of $\mathrm{k}$. When coupled to $S_{\mathrm{ij}}^{\mathrm{L}}$, this feature represents the ability of the large structures of the flow (associated with small $\mathrm{k}$ ) to reach out and influence remote parts of the flow while the influence due to the smaller structures of the flow (associated with high $\mathrm{k}$ ) remains highly localized.

Now we couple the local source term, $S_{i j}^{L}\left(y^{\prime}, k, t\right)$ to the reaching term, $Q\left(y^{\prime}, y, k, t\right)$ and show how the coupling results in a smeared out nonlocal source, $S_{\mathrm{ij}}^{\mathrm{NL}}(\mathrm{y}, \mathrm{k}, \mathrm{t})$, for $\partial \mathrm{R}_{\mathrm{ij}}(\mathrm{k}) / \partial \mathrm{t}$ that extends past the edges of the TMZ in physical space. Figure 6.3.3 represents this coupling for a generic value of $k$.

As before, the smooth hump-like figure represents the local source (figure on top), and the new feature to notice is how this local source is smeared out past the edges of the TMZ (figures on bottom). The points at the various y positions outside of the TMZ are used to show the form of the Q-function. (Only a few points are included for clarity). The point to be made with Fig. 6.3.3 is similar curves for $Q$ exist for all values of $y$ outside of the TMZ, which become more narrow at the base for the larger values of $k$ and widen at the base for smaller values of $\mathrm{k}$. As the integral in the nonlocal source term is performed, contributions 
are made to $\partial \mathrm{R}_{\mathrm{ij}}(\mathrm{k}) / \partial \mathrm{t}$ at positions that lie outside of the $\mathrm{TMZ}$ due to the $\mathrm{Q}$ function "reaching" into the TMZ.

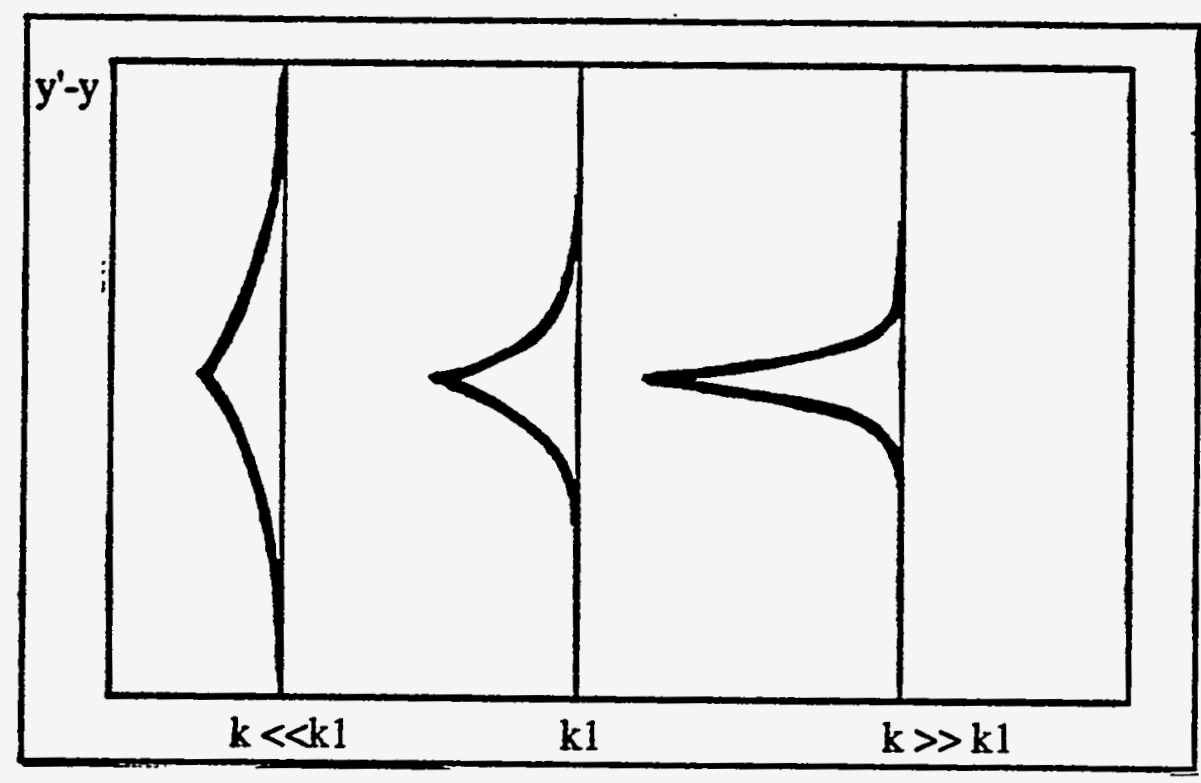

Figure 6.3.2. Schematic representation of the shape of the $\mathrm{Q}$ function. Notice how the base in the $y$ direction becomes more narrow for larger values of $k$. The area contained by each curve is the same due to normalization.

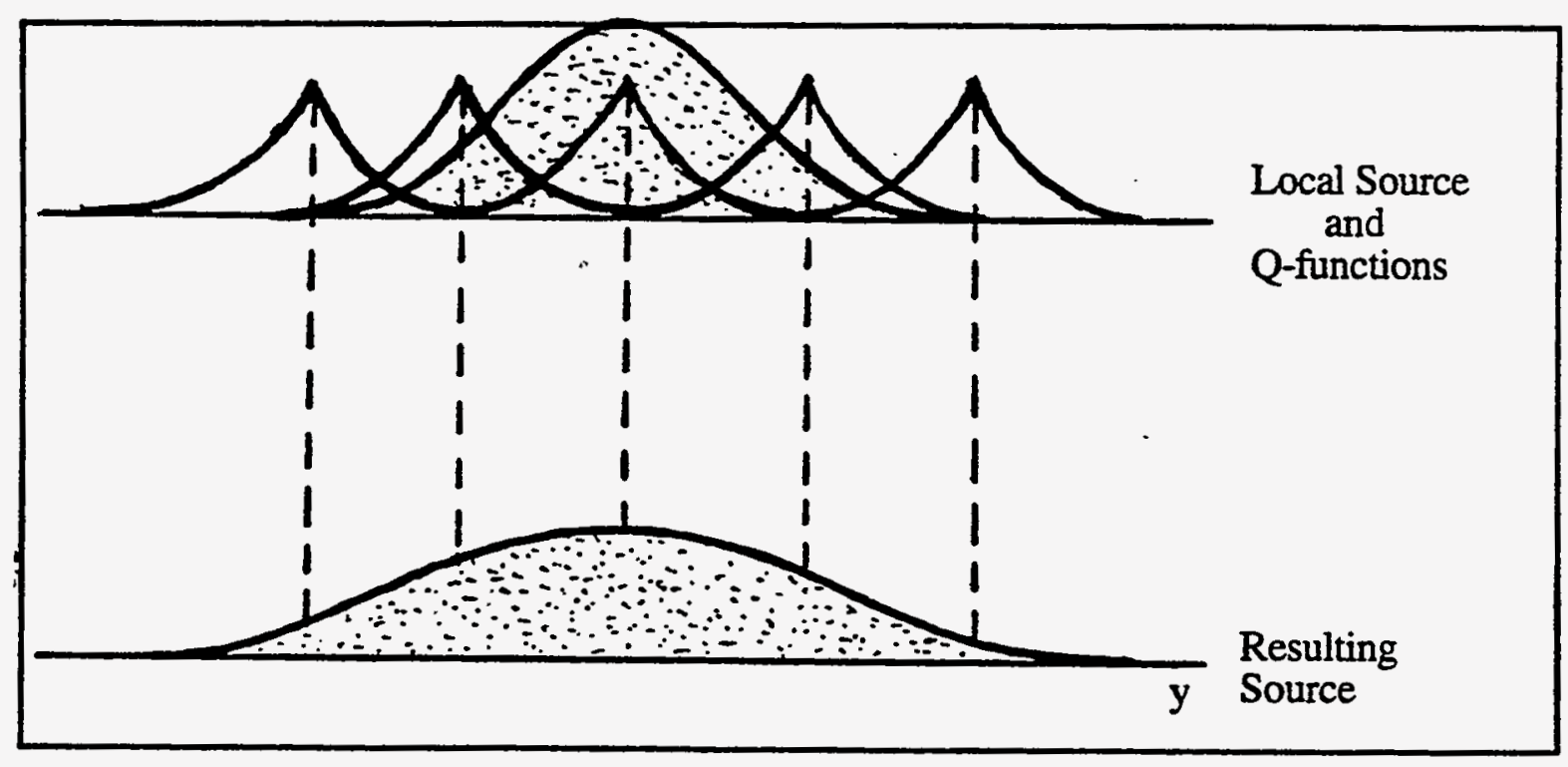

Figure 6.3.3. Schematic representation of the effect of the Q-function on the local source, $S_{\mathrm{ij}}^{\mathrm{L}}$, to $R_{\mathrm{ij}}(\mathrm{k})$ for a generic wave numbers. 
Figures 6.3.2-6.3.3 show the effect of coupling the Q-function to the local source term to create a nonlocal source term. It is shown that as $\mathrm{k}$ gets larger, the width of the base of the $\mathrm{Q}$ function becomes more narrow which retards the ability of the nonlocal source term to reach out past the edges of the TMZ. In this limit, the Q-function spreading has little physical basis, and we require only that the result for large $\mathrm{k}$ reduce to the local source. Recall that the extremum of the $a_{i}$ spectrum is at $k=k 1$. The consequence of this feature of the $a_{i}$ spectrum is that the effective local source for $\partial R_{i j} / \partial t$ will be larger for the wave numbers near $\mathrm{k} 1$. The effect of the nonlocality is to flatten the peak of the source near the middle of the TMZ and to extend the source past the edges of the TMZ. The peak of the nonlocal source at the center of the TMZ will always be lower than the peak of the local source due to the normalization,

$$
\int_{-\infty}^{+\infty} Q\left(y^{\prime}, y, k, t\right) d y^{\prime}=1 .
$$

For small wave numbers, the resulting nonlocal source is spread out over a relatively greater distance in physical space than for the larger wave numbers. This is to weight the nonlocal influence of the larger structures of the flow. Thus as we traverse the spectrum from $k=0$ to very large values of $k$, the $a_{i}$ spectrum starts at zero, reaches a maximum and then asymptotes back to zero; the effect of the nonlocal $Q$ function on the source is to reach out to infinity for $\mathrm{k}=0$ and asymptote to a local form for very large values of $\mathrm{k}$. When coupled, these two effects produce a source that (1) is small in magnitude and extends well past the edges of the TMZ in physical space for small values of $k,(2)$ is large in magnitude and is more restricted in physical space for intermediate values of $k$, and (3) is small in magnitude and completely confined to the TMZ in physical space for asymptotically large values of $\mathrm{k}$.

To summarize Figs. 6.3.2-6.3.3, examine Fig. 6.3.4 which compares the local and nonlocal shapes of the $\partial \mathrm{R}_{\mathrm{ij}} / \partial \mathrm{t}$ source term as a function of $\mathrm{y}$ for three different ranges of $\mathrm{k}$. 
The three plots of Fig. 6.3.4 show both the local and nonlocal source as a function of $y$ for different ranges of wave number. The main features of the plots are the decrease in the maximum of the source due to the nonlocality and the distance the nonlocal source extends past the local source as a function of wave number. These three plots of Fig. 6.3.4 can now be used to identify spectral modifications near the center and edge of the TMZ. A vertical strip is drawn through the edge of the TMZ as well as the middle of the TMZ $(y=0)$ to help identify how the nonlocal source term modifies the spectral behavior of $\partial \mathrm{R}_{\mathrm{ij}} / \partial \mathrm{t}$. The effect at the edge of the TMZ due to the nonlocality in the source term for $\partial \mathrm{R}_{\mathrm{ij}} / \partial \mathrm{t}$ is an increase in curvature for the small wave numbers, an increase in the absolute magnitude of the source as well as a migration of $\mathrm{k}_{\max }$ to lower wave numbers. The effect at the center of the TMZ due to the nonlocality in the source term for $\partial \mathrm{R}_{\mathrm{ij}} / \partial \mathrm{t}$ is a decrease in the curvature of the spectrum at the lower wave numbers, a lower absolute magnitude of the source at the centerline, and a migration of $\mathrm{k}_{\max }$ to higher wave numbers. This is shown in Fig. 6.3.5.

The information from Fig. 6.3.5 can now be used to determine qualitatively the effects of a nonlocal source on the behavior of $k_{\max }$ through the TMZ. The local source produces a $k_{\max }$ that is concave upward, as a function of $y$, consistent with the largest turbulent length scales at the centerline of the TMZ, (Scale $\left.=1 / \mathrm{k}_{\max }\right)$. The nonlocal source produces a $\mathrm{k}_{\max }$ curve through the TMZ that is concave downward, consistent with the larger turbulent length scales found at the edges of the TMZ. Inspection of Fig. 7.4.5(c) and (d) indeed verifies this behavior.

In the limit as $\mathrm{k} \rightarrow 0$, the value of $\mathrm{n}$ is preserved at the initial magnitude. From the nonlocal source term for example with $R_{n n}$ going as $k^{n} \exp [2 t \sqrt{k(A t) g}]$ for $k$ sufficiently small, this becomes, to leading order,

$$
\mathrm{k}^{\mathrm{n}}\{1+2 \mathrm{t} \sqrt{\mathrm{k}(\mathrm{At}) \mathrm{g}}+\ldots\}
$$

which shows the preservation of $\mathrm{k}^{\mathrm{n}}$ in the limits as $\mathrm{k} \rightarrow 0$ and also the emergence of a $\mathrm{k}^{\mathrm{n}+1 / 2}$ contribution in the spectra from near $k=0$. This effect can only be visible if $\mathrm{C}_{\mathrm{fb}}$ is 
sufficiently small, considerably less than that which is required for agreement with experiment.

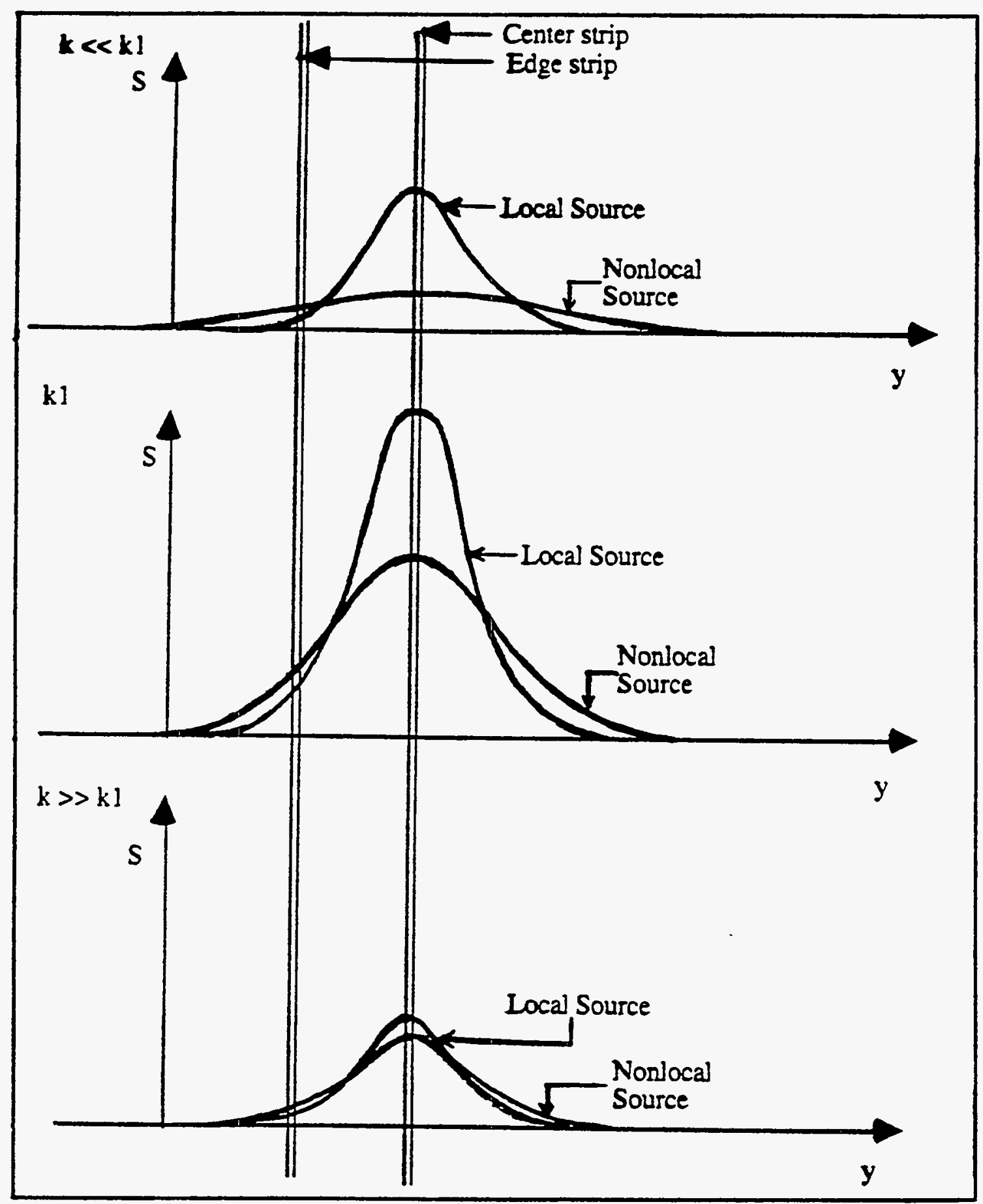

Figure 6.3.4. Schematic to represent the different structures through the TMZ of the nonlocal source term for $\partial R_{i j} / \partial t$ due to the dependence of the nonlocality on wave number $k$. 


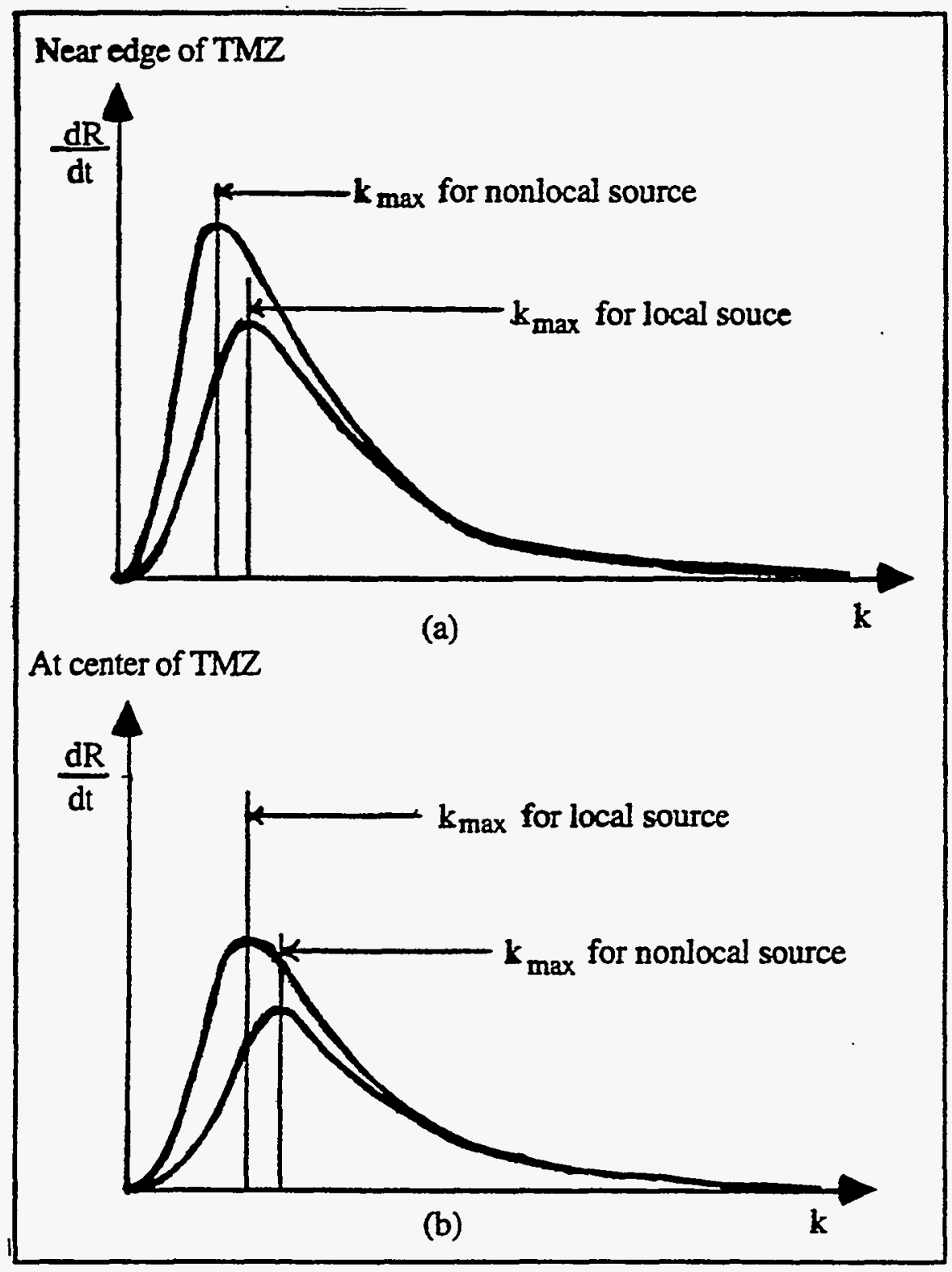

Figure 6.3.5. Schematic representation of the spectral modifications to the time rate of change of the Reynolds stress tensor due to a nonlocal source term for: (a) the edge of the TMZ and (b) the center of the TMZ.

In summary, the nonlocal source term for $\mathrm{R}_{\mathrm{ij}}$ affects the spectrum differently as we traverse the TMZ. Near the centerline of the TMZ the power, $n$, which dictates the power law behavior of the spectrum, $\mathrm{k}^{\mathrm{n}}$, is decreased for the low wave numbers and only slightly changed at the high wave numbers. Near the centerline, the $\mathrm{k}_{\max }$ migrates towards higher wave numbers. As we move out towards the edge of the TMZ, the power $n$ is increased for 
the lower wave numbers, the higher wave numbers are only slightly affected, and $\mathrm{k}_{\max }$ migrates to the lower wave numbers. The nonlocal source term for $\mathrm{R}_{\mathrm{ij}}$ has a major effect on the components of that tensor and is also felt indirectly on $a_{i}$ and $b$, principally through the alteration of cascade rates.

Two more items that alter the spectra for the low wave numbers are the initial conditions of $b$, i.e., the low wave number initialization for the $\mathrm{b}$ spectrum, and the $\mathrm{C}_{\text {fb }}$ term in the $\mathrm{b}$ equation. The low wave-number behavior for the $\mathrm{b}$ spectrum and the $\mathrm{C}_{\mathrm{fb}}$ are in constant competition to determine the power law behavior for the low k parts of the spectra. This competition has the greatest influence within the $b(k)$ spectrum itself. The $C_{f b}$ term is responsible for a wave-like propagation of the $b$ spectrum from high to low wave numbers. This effect will increase the downward curvature of the $b$ spectrum for the low wave numbers which lowers the $\mathrm{n}$ of the spectrum where $\mathrm{b} \sim \mathrm{k}^{\mathrm{n}}$ for small $\mathrm{k}$. Hence it is apparent that this process will compete with the initial condition of the b spectrum since the spectrum is continually migrating toward smaller wave numbers, to allow for a continual influence of the initial conditions. The effect of the $\mathrm{C}_{\mathrm{fb}}$ term, however, is to always lower the value of $\mathrm{n}$ for the low wave number parts of the b spectrum. The outcome of the competition of these two items then determines the low wave-number behavior for the $a_{i}$ spectrum that in turn influences the $R_{i j}$ spectrum.

One final class of terms to be mentioned that also alter the spectra at the low wave numbers are the $C_{2}$ cascade terms in each of the equations (the $C_{1}$ cascade terms are wavelike propagation to high wave numbers and have their principal manifestations at the high end of the spectrum). The $\mathrm{C}_{2}$ cascade terms alter the spectral shape at a rate that depends on $R_{n n}(k)$. The alteration of this rate is thus affected by the nonlocal source, but this effect is considered to be secondary. The primary effect of the varying rate comes simply from the decrease in $R_{n n}$ with y for either type of source. 


\subsection{Summary}

In this chapter we presented two separate discussions: (1) a detailed analysis of the derivation of the nonlocal pressure source term for the Reynolds stress transport equation (as described in Section 5.3) due to the effects of incompressibility and (2) an investigation of how this nonlocal formulation alters the spectral behavior of our model and how the additional complexities of inhomogeneity and variable density tend to alter the spectra as compared to the case of constant density homogeneous turbulence.

In Section 6.2 we demonstrate the strategy we use in deriving a nonlocal source term for the Reynolds stress tensor transport equation. We appeal to the linear analysis of the Rayleigh-Taylor instability as guidance in deriving a function that will spread the effects of the source term past the edges of the TMZ. The unmodeled source term is made up of a density--velocity correlation $\left(a_{\mathfrak{i}}\right)$ coupled to the mean pressure gradient.

In Section 6.3 we use schematic drawings to explain the effects of this nonlocal spreading function, $\mathrm{Q}\left(\mathrm{y}, \mathrm{y}^{\prime}, \mathrm{k}\right)$, on the spectral behavior of the model. In Section 6.3 we also discuss the various other alterations of the spectrum due to terms in this model. We use the case of constant density homogeneous turbulence as a reference to demonstrate the spectral alterations. 


\section{Numerical Results for the Spectral Turbulence Transport Equations}

\subsection{Introduction}

In this section, we present the numerical results computed with the spectral equations from Section 5.3 as applied to turbulent mixing by the Rayleigh-Taylor instability. The purpose of this section is to demonstrate the behavior of a base case of the spectral equations of Section 5.3, show comparisons with experiments, and show the effect of some variations to the base case. For this demonstration, we will present numerical results of a base case for mixing only, followed by examples to demonstrate the influence that the various coefficients have on the calculations. We also show the spectral behavior for the net mass flux velocity, a, during the process of a rapid transient (a gravity reversal is used for this demonstration).

To study the properties of the spectral equations we have written a finite-difference code for their numerical solution. The configuration we have chosen for illustration is that of an initial interface between two nonviscous, incompressible fluids of different densities, subjected to a pressure gradient normal to the interface. The pressure gradient arises by applying a body force (acceleration) to the fluids but holding the containing vessel at rest. An equivalent procedure, employed by the experiments with which we compare, accelerates the vessel, with a pressure gradient arising in order that the fluid is accelerated commensurate with the motion of the vessel.

The numerical solution technique and its validation for the present purposes is described in Appendix F. The computer code consists of a spectral part and a spectrallyintegrated part. In the latter, with the containing vessel at rest, the pressure gradient is updated in such a manner as to ensure vanishing flux of material volume at all stations within the flow. Thus the transport equations for $\tilde{\mathbf{u}}$ and the spectrally integrated transport equation for a are forced to be equivalent, which determines the variations of pressure in response to the specified body force. 
The principal data for comparison with calculations comes from extensive experimentation at AWE (Smeeton and Youngs, 1987) and Chelyabinsk-70 (Kucherenko, et al. 1991). The data are not as complete as we would like; we expect considerable more data from the anticipated experiments of Dimonte and Remington at LLNL (personal communication, 1995). The results currently available are profiles of estimated mix fraction across the layer, the mix interpenetration rates into each fluid, and some qualitative indications of dominant scale.

\subsection{Mixing due to an acceleration}

Figures 7.2.1-7.2.12 give results from a base-case calculation. In the following figures, the spectral behavior of the variables is plotted as functions of the nondimensional variable $\mathrm{z}$, where $\mathrm{z}=\mathrm{Ln}\left(\mathrm{k} / \mathrm{k}_{0}\right)$. This logarithmic transformation is used to accentuate the regions of physical significance; that is to say, as the turbulence evolves the turbulent length scales grow, which corresponds to a migration of the turbulence spectra toward $\mathrm{k}=0$. Thus the transformation allows the details of the spectrum near $\mathrm{k}=0$ to be spread over the corresponding infinite interval in nondimensional $z$-space. The constant reference wavenumber $\mathrm{k}_{0}$ serves to nondimensionalize the transformed spectral equations and to provide a reference wave number for specifying initial conditions. The time and length scales referred to in this chapter are set by the reference wavenumber $\mathrm{k}_{0}$ and the acceleration g. The initial configuration consists of a heavy fluid $(\rho=2.0)$ sitting over a lighter fluid $(\rho=1.0)$. At the time $t=0$, acceleration $(g=-1.0)$ is turned on, and the mixing of the two fluids commences. For this calculation, the values of the coefficients are $C_{1}=0.1212$, $\mathrm{C}_{2}=0.0606, \mathrm{C}_{\mathrm{fb}}=0.5, \mathrm{C}_{\mathrm{D}}=0.03, \mathrm{C}_{\mathrm{rp} 1}=5.0, \mathrm{C}_{\mathrm{rp} 2}=6.0, \mathrm{C}_{\mathrm{m}}=1.0$, and $\mathrm{C}_{\mathrm{bd}}=0.0$. The nonlocal version of the source term is used in the Reynolds-stress equations. At $t=0$, the values of $R_{n n}(k), R_{y y}(k)$, and $a(k)$ are zero in every computational cell. The value of $b(k)$ likewise vanishes in every cell except the one containing the two-fluid interface, where 


$$
b(k)=\frac{\gamma_{1} k^{n}}{1+\gamma_{2} k^{n+5 / 3}}
$$

The two coefficients, $\gamma_{1}$ and $\gamma_{2}$, have been chosen to ensure that the maximum of $b(k)$ occurs at $k=k_{0}$ and the spectral integral of $b(k, y)$, namely $b(y)$, has the maximum configurational value corresponding to equal volume fractions, $\alpha_{1}$ and $\alpha_{2}$, of the two fluids in that cell, i.e., $\alpha_{1}=\alpha_{2}=0.5$,

$$
\mathrm{b}=\frac{\left(\rho_{2}-\rho_{1}\right)^{2}}{4 \rho_{1} \rho_{2}} .
$$

See Chapter 2 for a discussion on the configurational value of $b$. For the base case, $n=4.0$, $\mathrm{k}_{0}=1.0$, and

$$
\gamma_{1}=\frac{\left(\rho_{1}-\rho_{2}\right)^{2}}{4 \rho_{1} \rho_{2} f(n)}
$$

and

$$
\gamma_{2}=\left(\frac{3 n}{5}\right) k_{0}^{-(n+5 / 3)}
$$

where,

$$
f(n)=\int_{0}^{\infty} \frac{k^{n} d k}{1+(3 n / 5)\left(k / k_{0}\right)^{n+5 / 3}}
$$

Boundary conditions in y space are of no significance because each calculation is terminated before the influence due to the nonlocal pressure effects have reached the edges of the computational mesh. Boundary conditions in $\mathrm{k}$-space attach the low and high wavenumber behavior for each variable to a power-law variation of $k$. For $b(k)$ we have also used a boundary condition at high wave numbers that blocks the cascade flux, but for the base case the flux is not blocked.

Figure 7.2.1 illustrates the time evolution of the spectral behavior for the variables as functions of the logarithm of $\left(\mathrm{k} / \mathrm{k}_{0}\right)$. In all four plots the spectra are migrating to the left (toward lower $\mathrm{k}$ values) as time progresses. We identify length scales as the reciprocal of the wave number at which the spectrum has its extremum. The leftward migration is thus consistent with an increasing length scale for each variable. The spectra are given for equal 
time increments, $\Delta t=10$, so as to demonstrate the linearly increasing growth rate of the peaks of the spectra. This variation is consistent with quadratic growth in time of the TMZ width, in agreement with expected self-similar behavior as described in Appendix D.

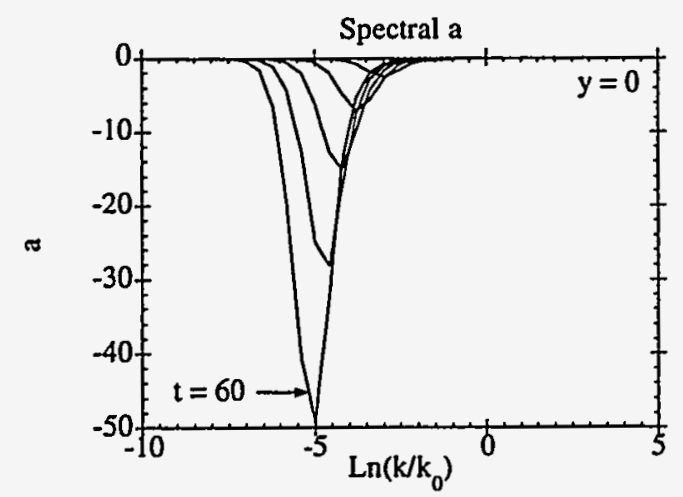

(a)

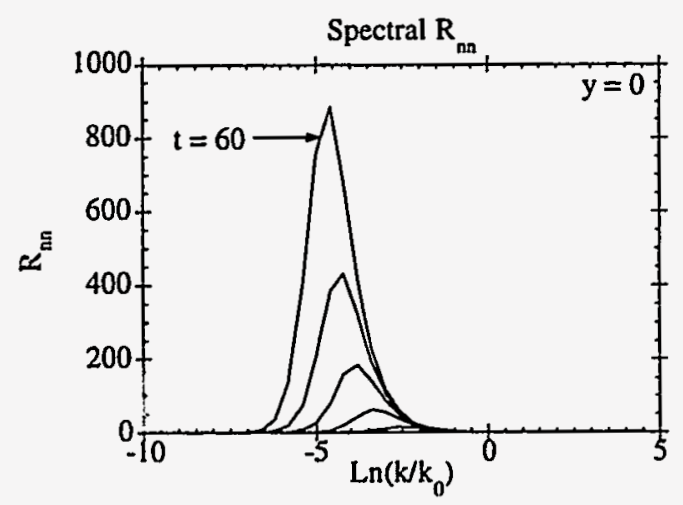

(c)

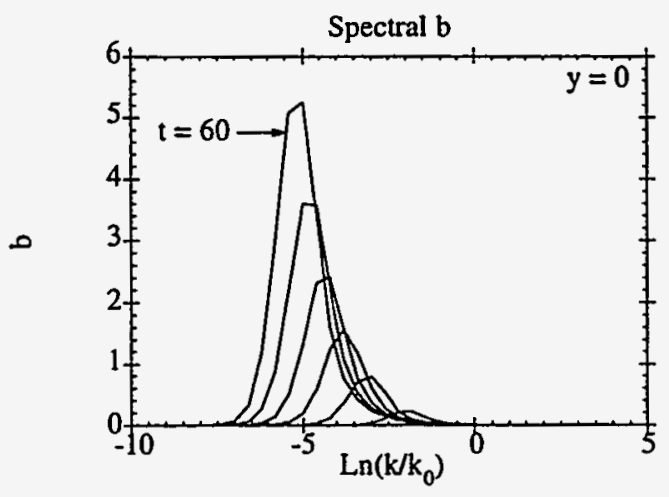

(b)

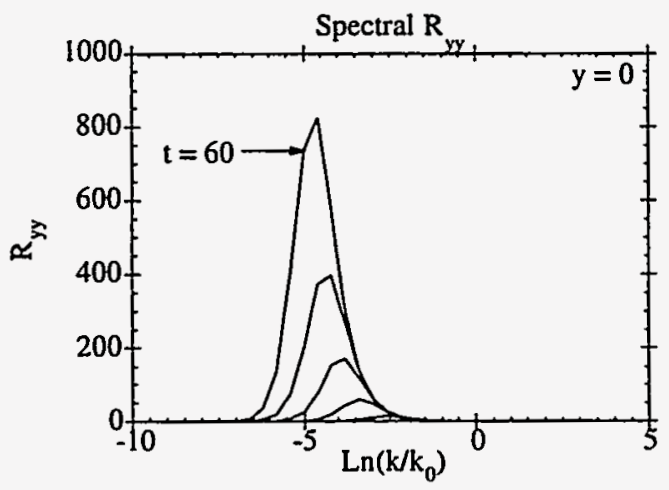

(d)

Figure 7.2.1. Spectra at times $t=10,20,30,40,50$, and 60 , at the position of the initial fluid interface: (a) the net mass-flux velocity, a, (b) the specific volume-density correlation, b, (c) the contraction of the Reynolds stress tensor, $R_{n n}$, and (d) the $R_{y y}$ component of the Reynolds stress tensor.

One characteristic that demonstrates the intervariable relationships that exist within the equations is the differing behavior of the spectral structures at the higher wave numbers. The right sides of the spectra for $a$ and $b$ migrate rapidly toward smaller values of $k$ causing an overlapping appearance for a sequence of plots, while the right sides of the spectra for $R_{i j}$ coincide for that same sequence of times. The strong migration to the left seen in the $b$ spectrum is due to the value chosen for $\mathrm{C}_{\mathrm{fb}}$, the one free parameter in our equations. The value of $\mathrm{C}_{\mathrm{fb}}$ significantly influences the leftward migration of the $b$ spectrum. This effect 
influences the $b$ spectrum the most. Because $b$ is a principal source to $a_{i}(k)$, the effect is also seen in the $\mathrm{a}_{\mathrm{i}}(\mathrm{k})$ spectrum but to a relatively lesser degree. The Reynolds-stress spectra are, in turn, also moved to lower wave numbers. The effects are associated with nonlinear bubble-doubling in the mixing layer, as observed in numerous experiments and directnumerical-simulation calculations, and are important for the agreement with experiments described below. These overall spectral forms result from a complex interplay among physical processes represented by the terms in the equations. In Section 6.3 we show in much more detail the origins for the behaviors described in this base-case calculation and its variants.

The self-similar nature of the spectral evolution of Fig. 7.2.1 can be demonstrated by plotting the results in the form of Fig. 7.2.2. The plots are normalized in space so as to enable us to compare the shapes of these plots at successive times. The resulting normalized plots are called shape plots. To achieve this normalization two steps are taken. First, a spectrum is divided by its extremum, resulting in all of the spectral maxima coinciding with 1.0 and values of the entire spectrum falling between 0 and 1 . Next, the spectra are all shifted such that the maximum of the spectra coincide with $k=k_{0}$. A sequence of times of these resulting shape plots are given on the same graph so as to identify any differences in their structures. The coincidence of all plots indicates a self-similar evolution of the mixing layer. The plots of Fig. 7.2.2 are taken from the cell where the initial fluid interface existed. The mixing evolution becomes self-similar near $t=30$, and this is demonstrated by the overlapping plots of this figure.

Notice the consequence of shifting the spectra so that their peaks coincide with $\mathrm{k}=\mathrm{k}_{0}$. As the spectral peak migrates to the left, less and less of the spectrum lies to the left of that peak and more of the resolved spectrum lies to the right. When the spectra are then shifted in the normalizing process, the appearance of a diminishing tail on the left side and an increasing tail on the right side is observed. Thus the plot with the longest tail on the left side represents the earliest time of the sequence and the plot with the longest tail on the right side 
represents the latest time in the sequence. The slopes at low and high wave numbers are given to demonstrate the behaviors of the spectral variables. The interpretation and significance of these forms and slopes is discussed in Section 6.3. It is especially noteworthy to observe here the very close achievement of self-similarity.

The slopes in Fig. 7.2.2 represent the power law behavior of the spectra at only the initial position of the fluid interface, $y=0$. Since these plots are functions of the logarithm of $\left(\mathrm{k} / \mathrm{k}_{0}\right)$, the slope of the line corresponds to the power, $\mathrm{n}$, of the wave number, $\mathrm{k}$. (These are slopes near $\mathrm{k}=0$ and represent modifying tendencies for low wave numbers near $\mathrm{k}=0$, but not at precisely $k=0$, where the initial value of $n$ is preserved.) The spatial variation of the value of $n$ across the TMZ for both the low and high wave numbers is shown in Fig. 7.2.3. This plot is given for $t=65$ well after the establishment of self-similarity. In effect, the spatial variations shown in Fig. 7.2.3 indicate the temporal evolution of the power laws for any one fixed position. The center of the plot $(y=0)$ has been developing the longest, and the edges of the TMZ are, relatively speaking, the newest parts. For the low wave numbers, the $b(k)$ spectrum has a smaller value of $n$ near $y=0$ than occurs for the other spectra. The explanation lies in the effects of the $C_{\mathrm{fb}}$ term, which gives to $b(k)$ the leading role in describing the nonlinear bubble-double process. Initialized with $\mathrm{k}^{4}$ variation near $\mathrm{k}=0$, the $\mathrm{b}(\mathrm{k})$ spectrum feels most strongly the modifying effects of leftward (in k space) propagation of a concave-downwards structure that decreases the exponent. With $b(k)$ as a source to $a(k)$, and $a(k)$ as a source to $R_{i j}(k)$, the decrement in exponent is successively less in these latter two functions. This behavior, and likewise, the behavior of both the $a(k)$ and the $R_{i j}(k)$ spectra is consistent with the overlapping seen in Fig. 7.2.1. The high wave number power law behavior of the four variables coincides at the edges of the TMZ and then separates as time evolves. The values of $n$ for the high wave numbers of Fig. 7.2.3(b) at $y=0$ are equal to the slopes of the plots in Fig. 7.2.2. 


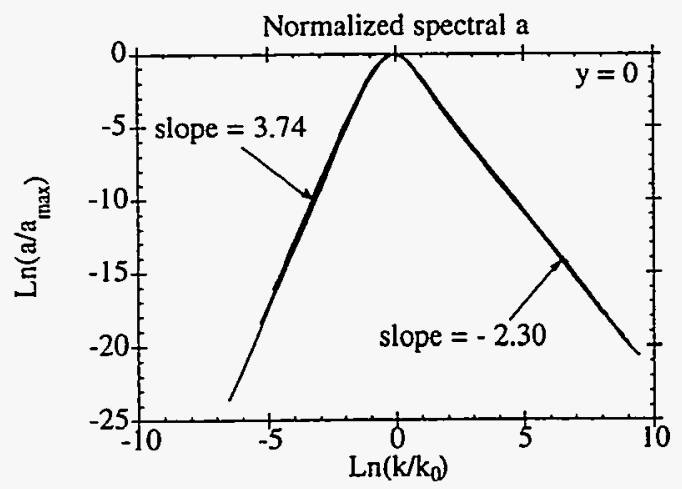

(a)

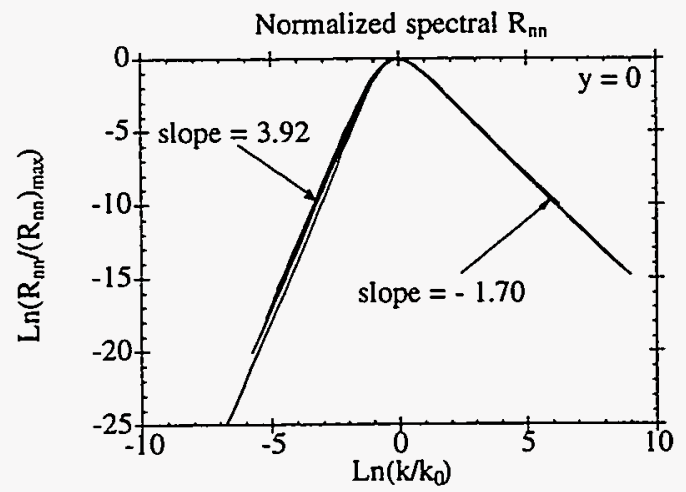

(c)

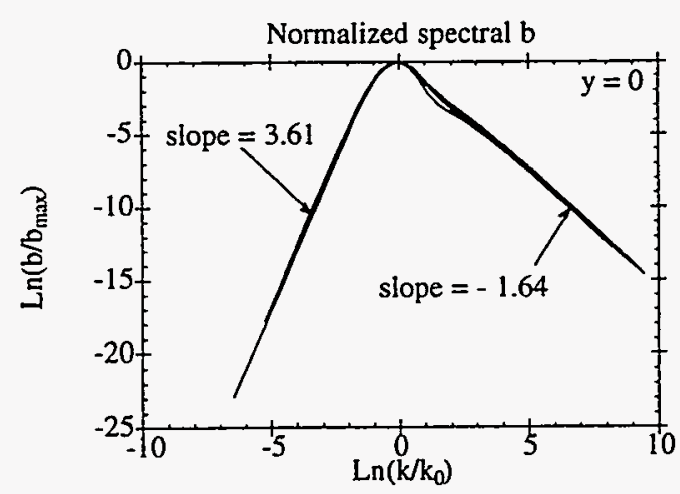

(b)

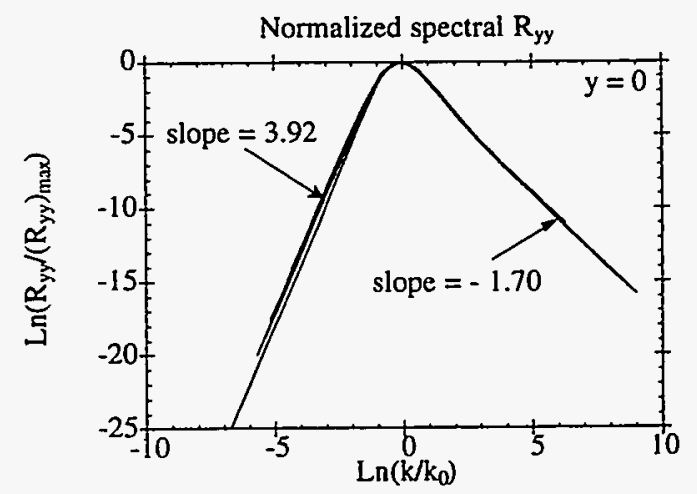

(d)

Figure 7.2.2. Normalized spectra for $\mathrm{t}=30,35,40,45,50,55,60$, and 65 , at the position of the initial fluid interface: (a) the net mass-flux velocity, a, (b) the specific volume-density correlation, b, (c) the contraction of the Reynolds stress tensor, $R_{n n}$, and (d) the $R_{y y}$ component of the Reynolds stress tensor.

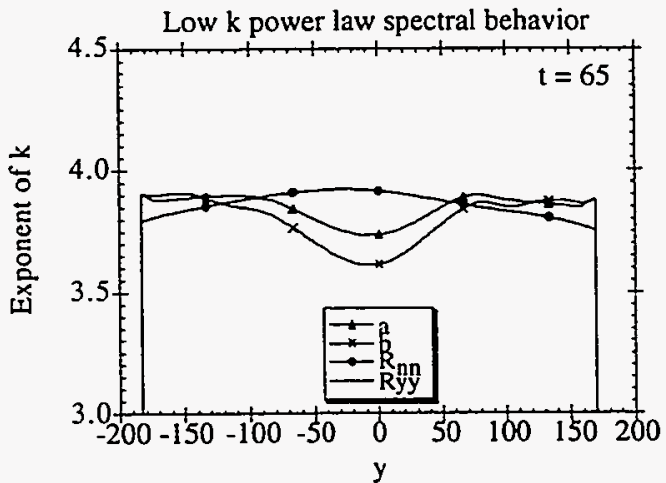

(a)

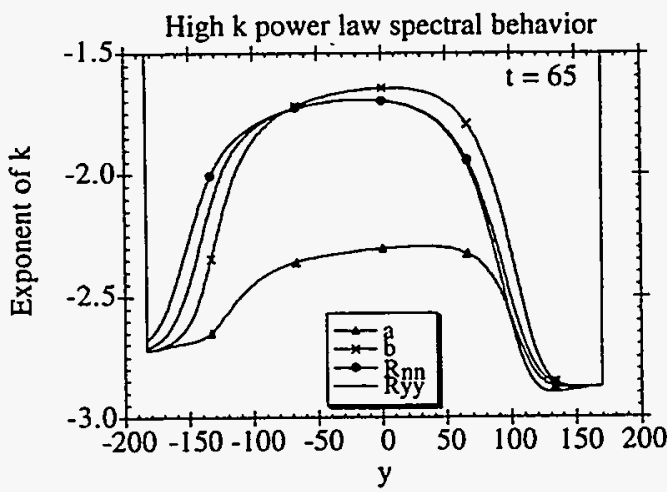

(b)

Figure 7.2.3. Profiles of the power law exponent across the TMZ at $t=65$ for (a) the low wave numbers, and (b) the high wave numbers. 
Only near $y=0$ does one observe the values of $n=-5 / 3\left[\right.$ for $b(k)$ and $\left.R_{i j}(k)\right]$ and $n=-7 / 3$ for $a(k)$, which result from the constancy of cascade flux for $b(k), R_{n n}(k)$, and $R_{y y}(k)$ and the balance of drag with production for $\mathrm{a}(\mathrm{k})$, as discussed in more detail in Section 6.3.

Figure 7.2.4 shows the evolving spectral forms at a distance of 50 units of length below the initial interface, showing the manner of arrival of the spectrum at each station as the TMZ widens. The spectrum of $a(k)$ arrives at nearly its self-similar form for all wave numbers, whereas the spectra for the other quantities arrives in self-similar forms only for the lower wave numbers; at the higher wave numbers there is significant lag in the establishment of spectral self-similarity. There are two reasons for this behavior. One of these is the effect of nonlocal source for $R_{n n}(k)$ and $R_{y y}(k)$, which projects the low-wave number effects into lateral regions well

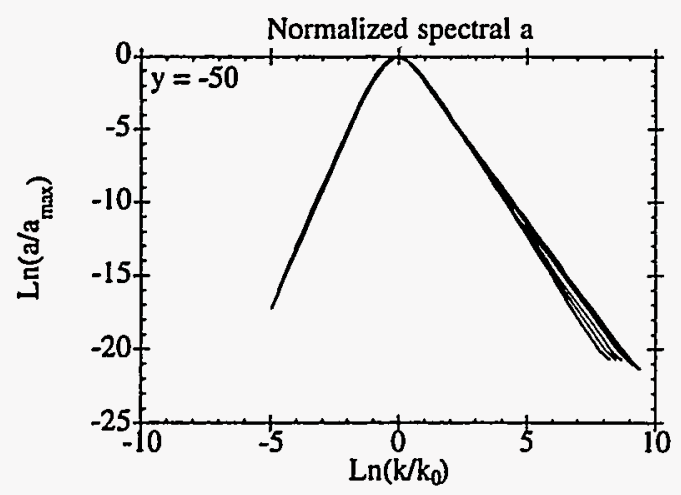

(a)

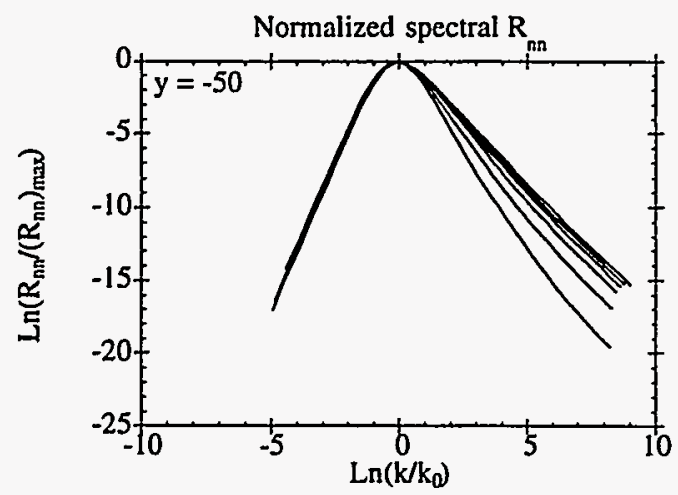

(c)

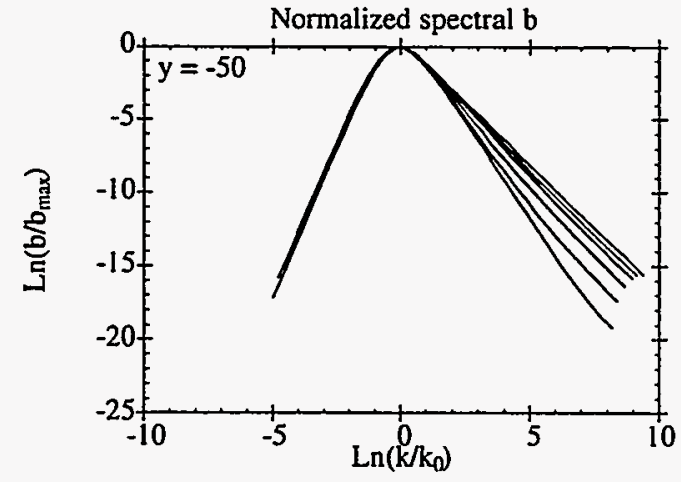

(b)

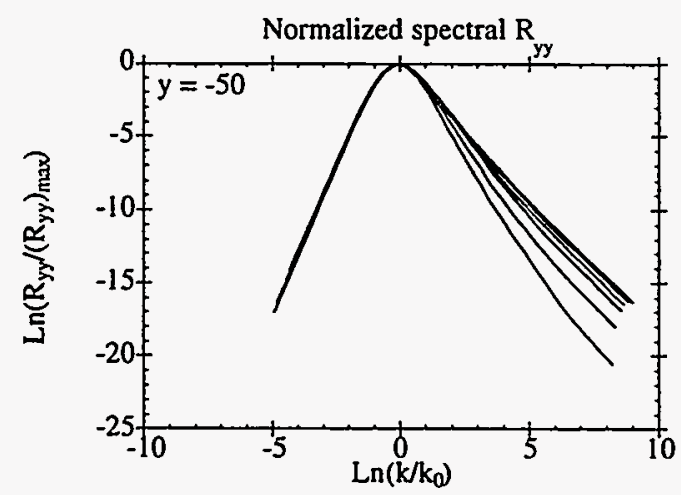

(d)

Figure 7.2.4. Normalized spectra for $\mathrm{t}=30,35,40,45,50,55,60$, and 65 , a distance of 50 units of length below the centerline of the TMZ: (a) the net mass-flux velocity, (b) the specific volume-density correlation, (c) the contraction of the Reynolds stress tensor, and (d) the $R_{\mathrm{yy}}$ component of the Reynolds stress tensor. 
ahead of the effects at high wave numbers. The second reason is that the growth of spectral forms for $b(k), R_{n n}(k)$, and $R_{y y}(k)$ is at first dominated by the effects of sources which at any station tend initially to impart a $\mathrm{k}^{-7 / 3}$ form at high wave numbers, and then altered to $\mathrm{k}^{-5 / 3}$ as the effects of constant cascade flux begin to dominate.

Figure 7.2.5 shows a comparison at $\mathrm{t}=65$ of the spectral form functions at $\mathrm{y}=0$ and $y=-50$. By this time the self-similar form has been completely reached at $y=0$ and nearly so at 50 length units below $y=0$. Their nearly identical forms serve as a confirmation of the hypothesis discussed in Appendix D. Figure 7.2.6 illustrates a similar comparison at $y=50$ units above the initial interface and again confirms the remarkable observation of the form function decomposition hypothesis described in Appendix D. This occurs only because of neglect of

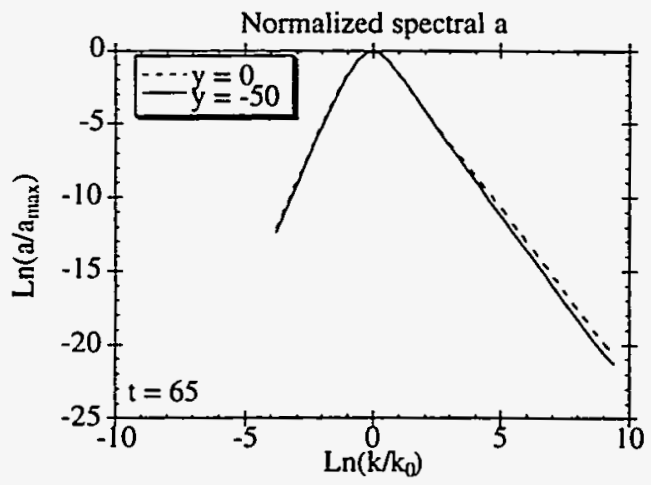

(a)

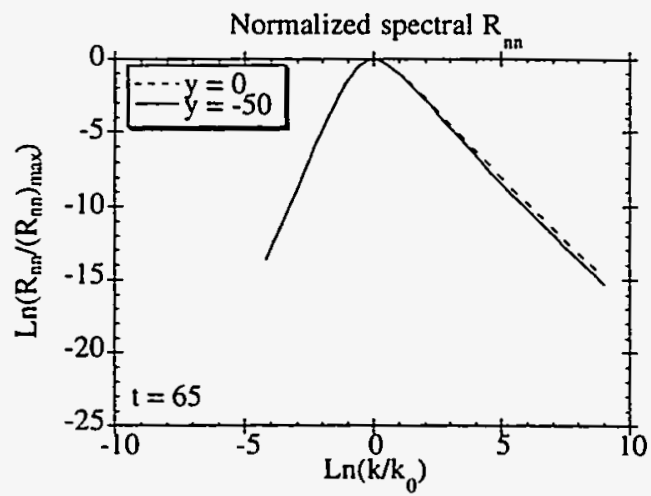

(c)

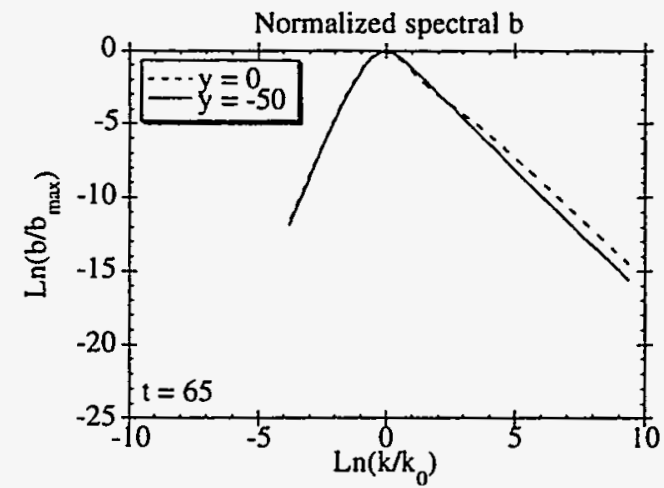

(b)

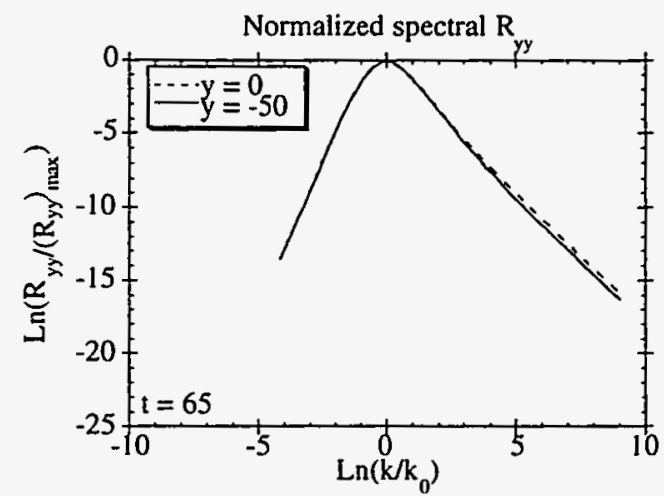

(d)

Figure 7.2.5. Comparison of normalized spectra at $y=0$ and $y=-50$ for $t=65$ for the variables: (a) the net mass-flux velocity, (b) the specific volume-density correlation, (c) the contraction of the Reynolds stress tensor, and (d) the $R_{y y}$ component of the Reynolds stress tensor. 
molecular effects and surface tension. It should be emphasized that the spectral normalizations to unit magnitude and shift to $k=k_{0}$ have occurred over very large and different ranges in these figures, so that their coincidence seems all the more remarkable. Because this behavior has also been observed in free-shear turbulence of a fluid with constant density (BHRZ, 1990; Clark, 1992) we believe that there is a powerful underlying scaling principle that can be exploited in the systematic analysis of certain turbulence circumstances that are inhomogeneous, anisotropic, and even have large variations in fluid density.

Figure 7.2.7 illustrates the nature of $\mathrm{k}$ shift that has been employed in getting the preceding plots of spectral form functions. In this figure we show the reciprocal of the wave number at

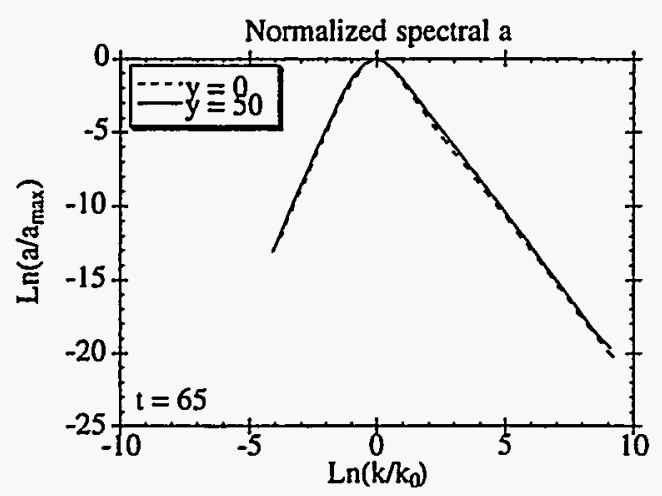

(a)

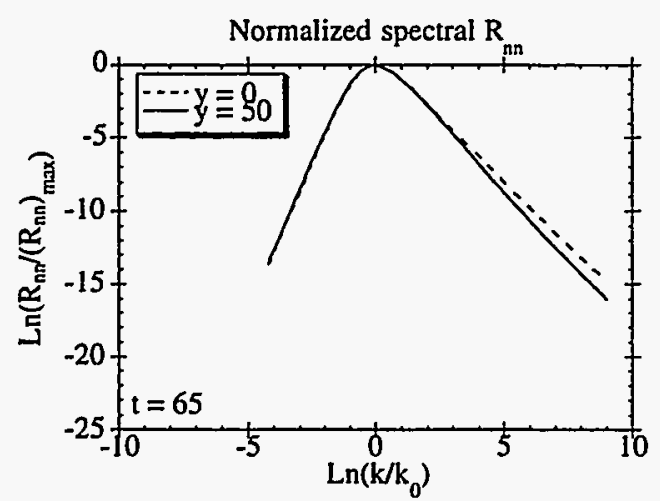

(c)

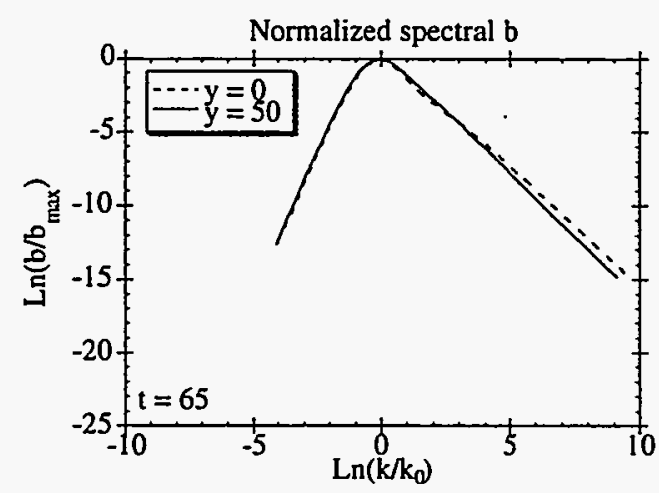

(b)

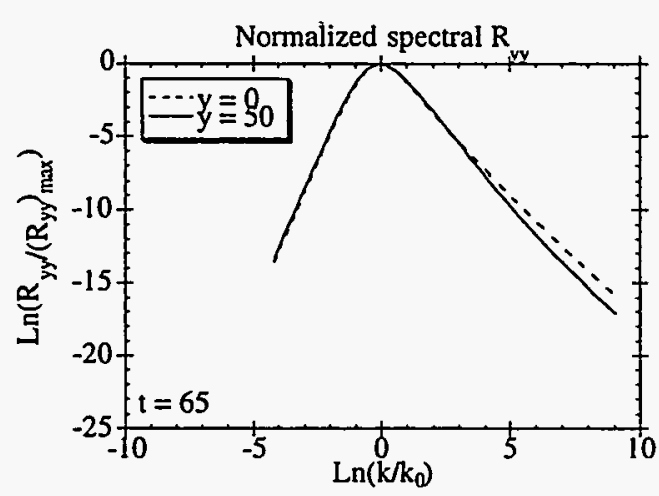

(d)

Figure 7.2.6. Comparison of normalized spectra at $y=0$ and $y=50$ for $t=65$ for the variables: (a) the net mass-flux velocity, (b) the specific volume-density correlation, (c) the contraction of the Reynolds stress tensor, and (d) the $R_{y y}$ component of the Reynolds stress tensor. 
which each spectrum has a maximum as a function of position across the TMZ. The ordinate is thus a length scale, which relates the length scale proportional to $\mathrm{K}^{3 / 2} / \varepsilon$ that occurs in single-point K- $\varepsilon$ turbulence transport models. Structural details in these lengthscale profiles have their origin in the initialization for calculations where we only initialize the one cell containing the original fluid-fluid interface, which is far from self-similar. These minor details, once established during the early-time adjustments to self-similarity, are captured and maintained by the achievement of that self-similarity, which indeed confirms the attainment of that state. Of more substance, however, are the overall forms of these plots. The length scales for $a(k)$ and $b(k)$ more-or-less decrease towards the edges of the mixing layer, whereas the length scales for $R_{n n}(k)$ and $R_{y y}(k)$ increase. The basic reason for the laterally increasing length scales for Reynolds

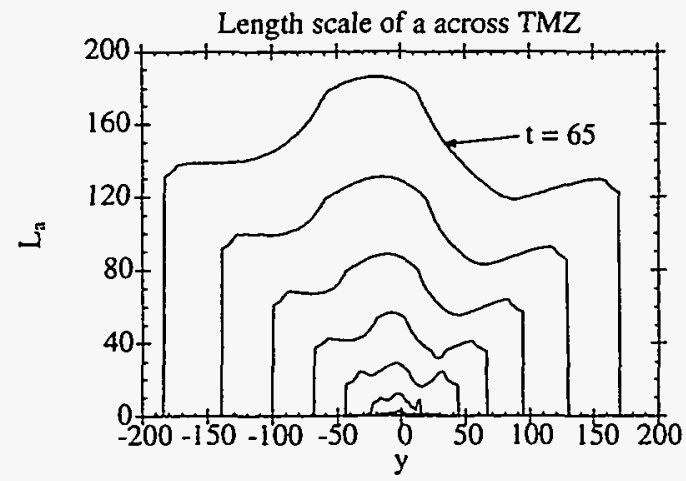

(a)

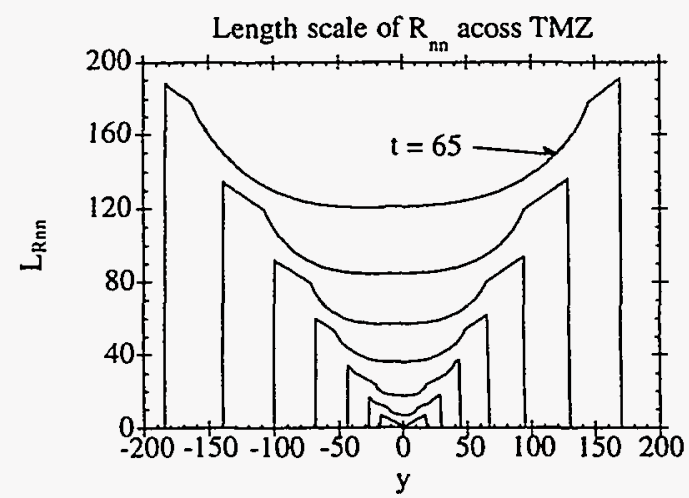

(c)

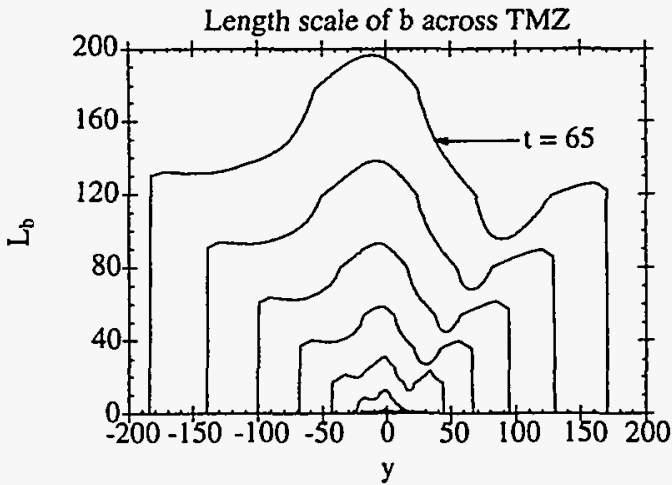

(b)

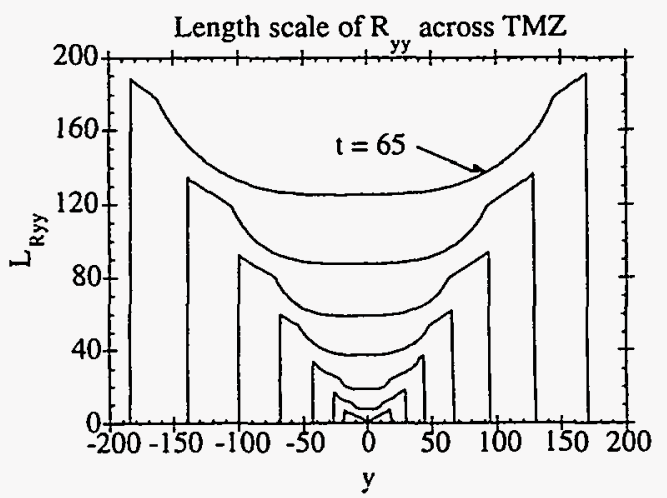

(d)

Figure 7.2.7. Profiles of the length scales across the TMZ for $t=5,15,25,35,45,55$, and 65: (a) the net mass-flux velocity, (b) the specific volume-density correlation, (c) the contraction of the Reynolds stress tensor, and (d) the $R_{y y}$ component of the Reynolds stress tensor. 
stress can be traced to the nonlocal source. This identification is confirmed by contrasting with the results for a local source term; see Fig. 7.4.5, which shows a lateral decrease in scale for $R_{n n}(k)$ and $R_{y y}(k)$. The significance of this effect for single-transport modeling is the lack of proportionality among length scales, for which we obtain remediation by allowing for more than one scale to arise in the moment derivations. (This generalization becomes even more significant in the nonself-similar response to rapid transients of drive.)

Self-similarity also occurs in physical space, as shown in Fig. 7.2.8, again confirming the hypothesis stated in Appendix D. The initialization of $b(k)$ at $y=0$ is seen as the spike at $t=0$. Lateral "wings" on the Reynolds-stress profiles arise as a consequence of the nonlocality of the creation term.

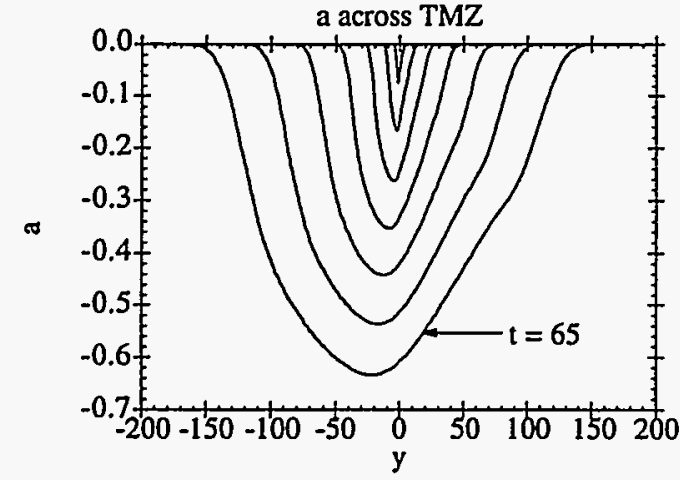

(a)

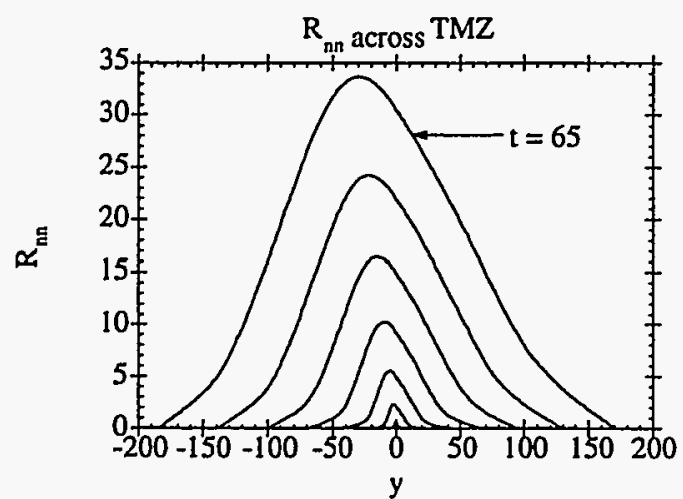

(c)

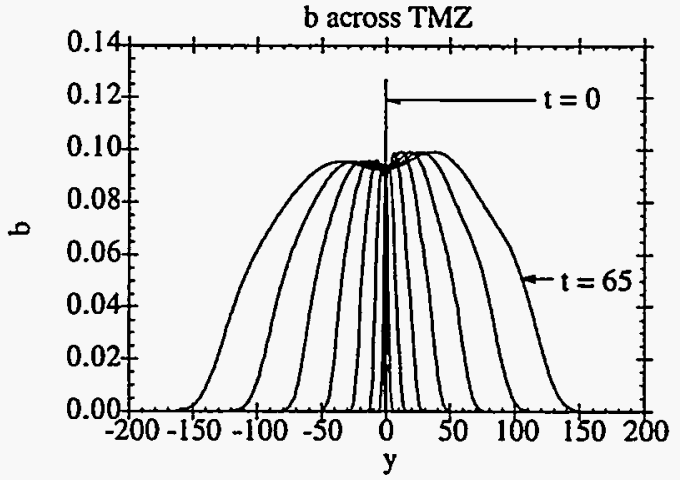

(b)

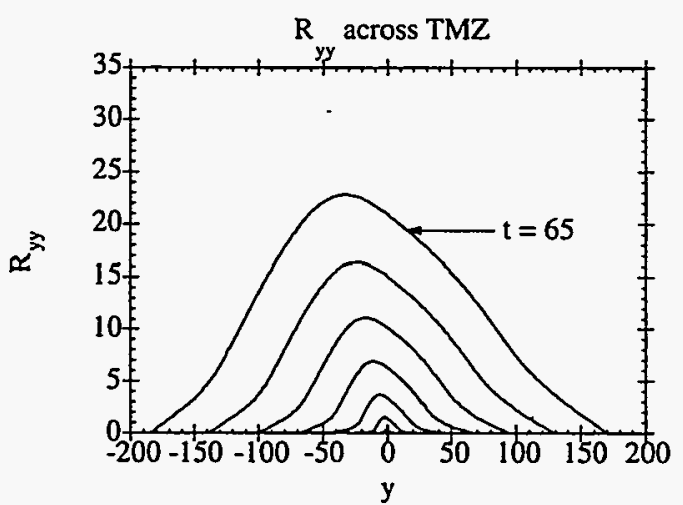

(d)

Figure 7.2.8. Profiles of spectrally integrated variables across the TMZ for $t=5,15,25,35,45,55$, and 65 : (a) the net mass-flux velocity, (b) the specific volume-density correlation, (c) the contraction of the Reynolds stress tensor, and (d) the $\mathrm{R}_{\mathrm{yy}}$ component of the Reynolds stress tensor. 
Figure 7.2.9 describes the numerical results in terms of observables that can be compared with experimental data. The principal data are the depth of penetration of heavy fluid into light, $\mathrm{h}_{2}$, of light fluid into heavy, $\mathrm{h}_{1}$, and the variations of relative concentration between these edges of the TMZ. In this figure we describe the concentration profiles in terms of mean density, $\bar{\rho}$ (Fig. 7.2.9(a)), together with the mass fraction and volume fraction of the heavier material (Fig. 7.2.9(b)). As pointed out by a Russian experimental group (Kucherenko, et al. 1991), the most convenient way to illustrate the profile shape is to plot volume fraction as a function of the reduced variable

$$
\Xi \equiv \frac{\mathrm{y}-\mathrm{y}_{0.1}}{\mathrm{y}_{0.9}-\mathrm{y}_{0.1}}
$$

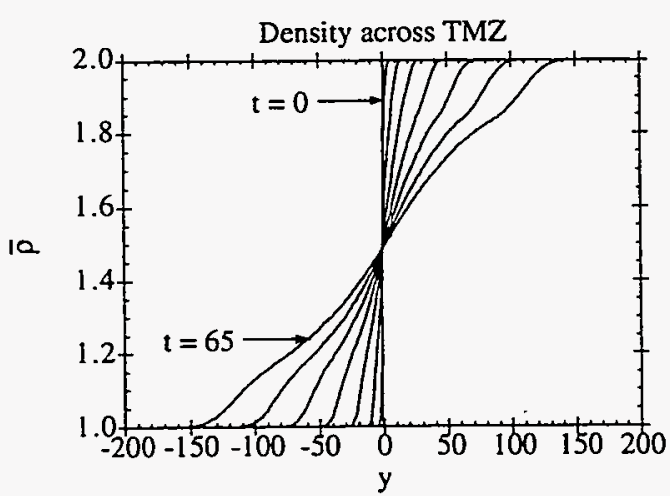

(a)

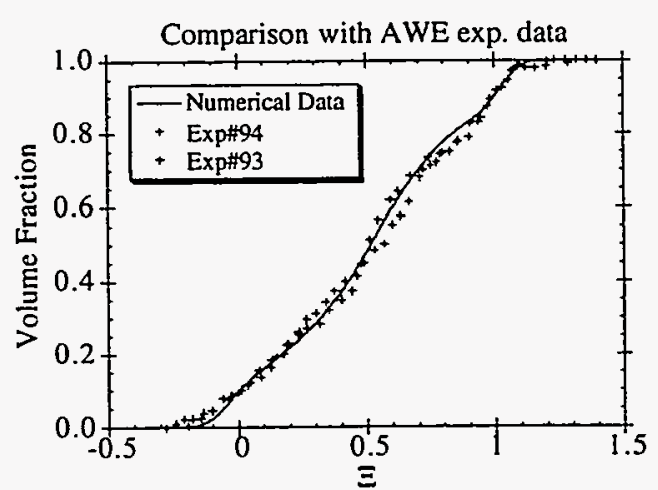

(c)

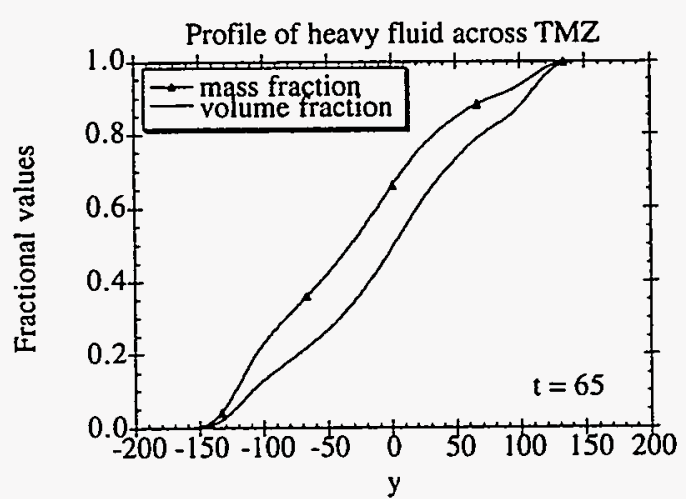

(b)

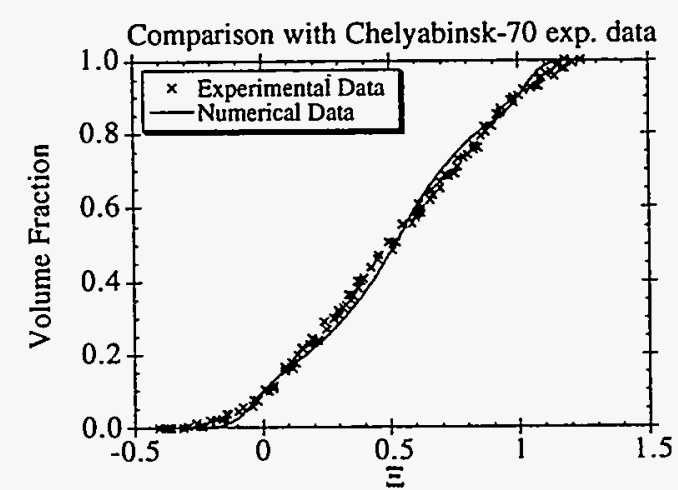

(d)

Figure 7.2.9. (a) Profiles of density across the $\mathrm{TMZ}$ for $\mathrm{t}=0,5,15,25,35,45,55$, and 65. (b) Mass and volume fractions of the heavy fluid across the TMZ at $t=65$. (c) Comparison of numerical results with AWE experimental data [Smeeton and Youngs, 1987 (Fig. 24 and 25)]. (d) Comparison of numerical results with Chelyabinsk-70 experimental data [Kucherenko, et al. 1991 (Fig. 11)] 
where $y_{0.1}$ and y 0.9 are the positions at which the volume fraction is 0.1 and 0.9 , respectively. The excellence of agreement with both the British (Fig. 7.2.9(c)) and Russian (Fig. 7.2.9(d)) experimental data is not as trivial as the simple structure of the profiles would suggest. Twofield transport theory also achieves this degree of agreement (see Chapter 4), but the singlepoint turbulence transport calculations using the BHR model has great difficulty in matching this simple profile of volume fraction across the TMZ without resorting to an ad hoc modification of the transport equation for the mass flux (see Chapter 3).

We freely admit here that we may still have some lingering modeling problems. The fact that the density gradient is not monotone through the TMZ remains a slight cause for concern. The single-point two-field equations described in Chapter 4 produce smooth density gradients through the TMZ. The experimental data from AWE and Chelyabinsk-70 seem to indicate a smooth behavior of the density. This detail remains as future work on improving this model.

The plots as functions of $\Xi$ in Fig. 7.2.9 exhibit the structure but preclude the observation of behavior for $h_{1}(t)$ and $h_{2}(t)$. These quantities are shown in Fig. 7.2.10 as functions of time and of the similarity variable,

$$
X=(A t) g t^{2}
$$

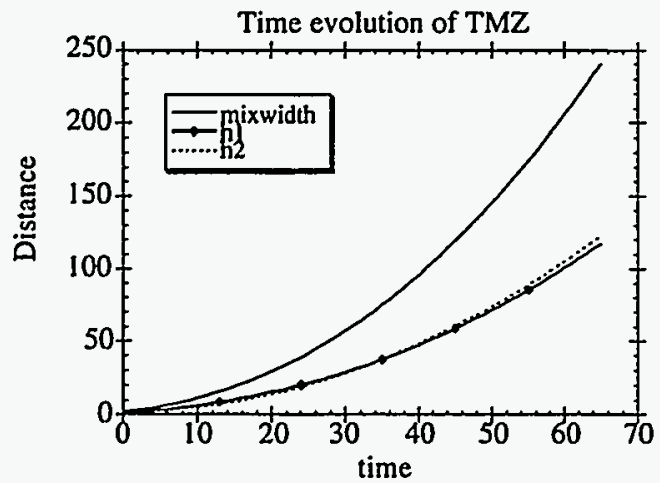

(a)

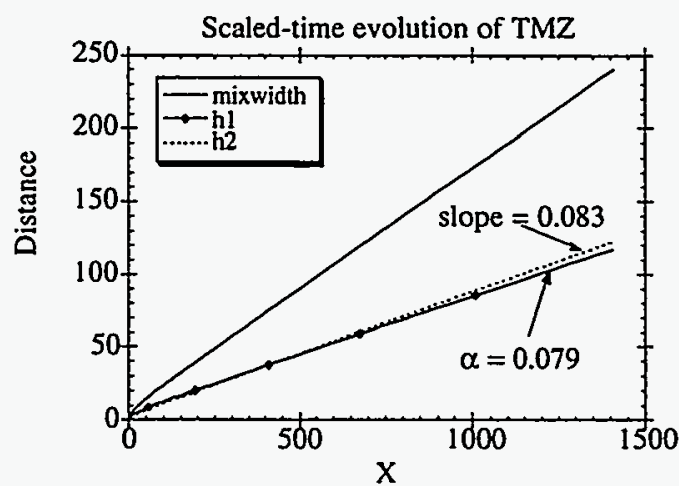

(b)

Figure 7.2.10. Spikes, $h_{2}$, and the bubbles, $h_{1}$, (a) as functions of time and (b) as functions of the similarity variable, $\mathrm{X}$. 
From an experimental viewpoint, the evolutions of $h_{1}$ and $h_{2}$ depend on how the edges of the TMZ are located. The same comment applies to the calculation results. As shown by the Russian experimentalists (see Sec. 7.4), edge criteria at volume fractions of 0.01 and 0.99 (fine-edge criteria) result in much larger values of $h_{1}$ and $h_{2}$ (and of $h_{2} / h_{1}$ ) than criteria at volume fractions of 0.1 and 0.9 (coarse-edge criteria). They also show and we confirm that the ratio $h_{2} / h_{1}$ converges very slowly to asymptotic value, being highly dependent on the early-time approach rate to self-similarity. They mitigate this difficulty by giving instead data for

$$
\frac{\mathrm{dh}_{2}}{\mathrm{dt}} / \frac{\mathrm{dh}_{1}}{\mathrm{dt}}
$$

which is calculated as the ratio of slopes in Fig. 7.2.10(b) and equals $h_{2} / h_{1}$ at late stages in the self-similarity. In Section 7.4 the calculated results are shown to lie a bit low for a nonlocal source and a bit high for a local source when compared with the data.

Self-similarity in our case means that the spreading of the TMZ can be characterized by

$$
\mathrm{h}_{1}=\alpha(\mathrm{At}) \mathrm{gt}^{2} \text {. }
$$

This expression for the height of the bubble envelope was empirically formulated from experiments by Read (1984) and Youngs (1989). There is experimental uncertainty to the value of the coefficient, $\alpha$, with quoted values anywhere from 0.02 to 0.3 and greater. Nikiforov (personal communication, 1994) speculates that the large range reflects the effects of miscibility of the fluids. For immiscible fluids there seems to be a fairly broad consensus that $\alpha=0.065 \pm 0.01$. Our calculated value, $\alpha=0.079$, is slightly higher than the currently accepted range.

An issue of some interest for our spectral equations is the rate of return to isotropy. Because the process is nonlinear and nonlocal in wave-number space, we have not examined the process in great detail. One point that can be mentioned is illustrated in Fig. 7.2.11, which shows the degree of anisotropy in the base-case calculation. As plotted versus wave number, $R_{n n}(k)$ and $R_{y y}(k)$ look nearly the same; whereas the spectrally integrated quantities 
plotted as a function of $y$ demonstrate that there is a significant difference between the two quantities. Note that if $R_{y y}=R_{n n}$, then $R_{x x}=R_{z z}=0$, and the behavior is completely anisotropic. If $\mathrm{R}_{\mathrm{yy}}=\mathrm{R}_{\mathrm{nn}} / 3$, then the three diagonal components of the Reynolds stress are equal and the configuration is completely isotropic. At low wave numbers the former condition prevails; at high wave numbers the complete isotropy is approached. Thus the turbulence is created at low wave numbers in a primarily anisotropic fashion and becomes progressively more isotropic at any fixed wave number as the TMZ grows and the spectrum shifts to the left. Interpenetration at the large scales (low wave numbers) is thus predominantly wave-like (hyperbolic, as in multifield flow), whereas at the finer scales (high wave numbers) the interpenetration becomes predominantly diffusive (parabolic, as in the more classical circumstances of "turbulence").

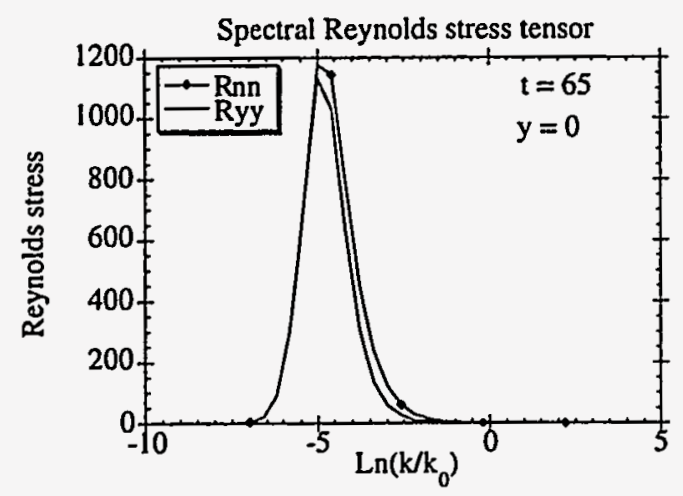

(a)

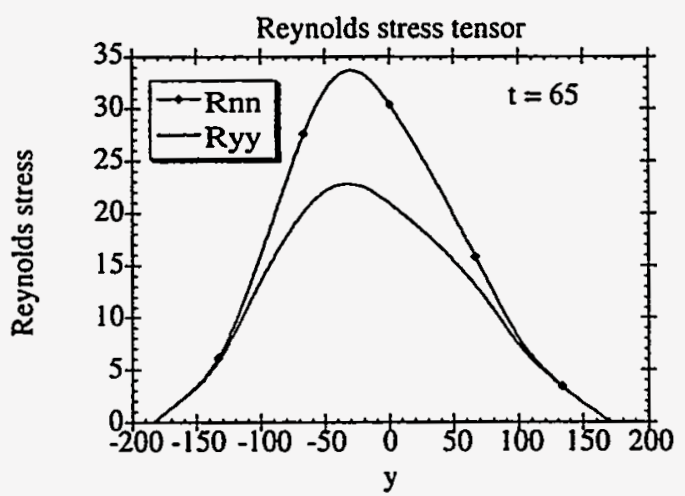

(b)

Figure 7.2.11. Comparison of the trace of the Reynolds stress tensor with $R_{y y}(a)$ at the centerline at $t=65$ and (b) in spectrally integrated form across the TMZ.

We now describe some calculations that depart in one or another respect from the base case. In the first of these variants, we investigated the consequences of blocking the cascade flux of $b(k)$ at a high wave number. Spectral integrals of $b(k)$ are shown in Fig. 7.2.12. With zero spectral flux, the cascade flux is conservative, and the integral now includes the passive parts of the spectrum that pile up at large wave numbers. The representation is a crude approximation to the behavior that would be expected from the 
presence of a smallest attainable scale that corresponds to a particle size that is not further subdividable. Whereas the $\mathrm{C}_{\mathrm{fb}}$ term describes an inverse transfer to progressively larger scales, the cascade to small scales shows turbulence effects in tearing down the clumps. With a large Schmidt number, the latter process would be expected to lead to the $\mathrm{k}^{-1}$ spectral behavior of a passive scalar for those clump scales whose wave numbers exceed the viscous cutoff. We have not investigated such circumstances.

Figure 7.2.12 shows that the added contribution to the spectral integral is noticeable, especially for the spatial positions (near the original interface) for which direct cascade has been proceeding long enough for a high-wave-number buildup. The most significant feature of this calculation is the negligible effect of blockage on the rest of the variables, not shown here because the differences are simply not visible on the plots. These observations motivate the identification of a passive region associated with the high wave numbers of the $b$ spectrum. We identify this region as passive because of its minor effects on the rest of the transported variables. One of the principal creation terms for $a(k)$ is the $b(k) d p / d x$ term. In this passive region, the creation of $a(k)$ due to $b$ is essentially balanced by the large drag of the small-scale clumps. This balance of creation with drag coupled with the fact that $a(k)$ behaves as $k^{-7 / 3}$ for large wave numbers renders this portion of the $b$ spectrum passive for mixing processes. The high wave number part of the $b$ spectrum becomes active once again during a rapid transient. The details of these interactions are demonstrated in Fig. 7.3.1 and discussed more thoroughly in Section 7.3. The configurational value of $b$ in the Fig. 7.2.12 is given in LA-12303-MS (Besnard, et al. 1992) as

$$
\frac{\alpha_{1} \alpha_{2}\left(\rho_{1}-\rho_{2}\right)^{2}}{\rho_{1} \rho_{2}} .
$$




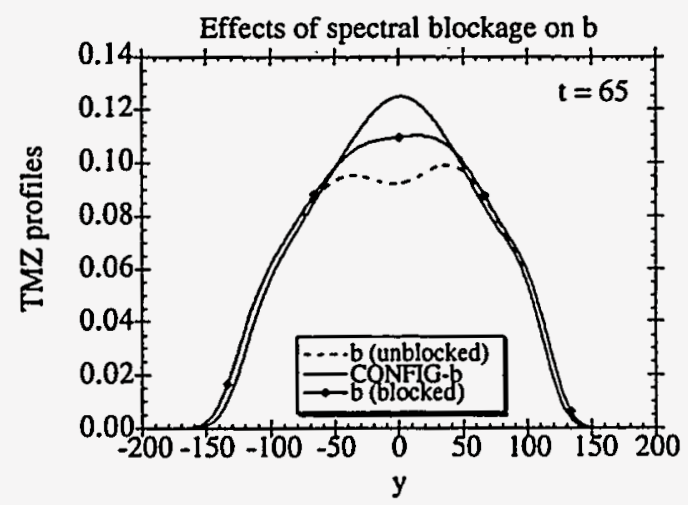

Figure 7.2.12. Profile of $b$ across the TMZ for the case of a spectrally unblocked calculation and a spectrally blocked calculation compared with the configurational calculation of $b$, at $t=65$.

The transported values of $b(k)$ follow the configurational form rather closely and are less near the center and somewhat higher in the lateral regions of the TMZ, principally as a result of diffusion in the transport equation, which is conservative in physical space. Some implementations of single-point turbulence transport equations (e.g., the BHR model) into computer codes use a purely configurational form for $b$, and our results are consistent with that procedure. Having demonstrated the close relationship between transported $b$ and configurational $b$, we wonder why not use purely the configurational form. The answer is in two parts.

- There is no configurational form for spectral $b(k)$, for which the distribution across wave-number space is needed for the spectral analysis.

- In the presence of phase transitions or molecular diffusion between the two fluids, the transport equation can be directly modified to include these effects, together with the resulting decay of $b(k)$ ultimately to zero. In the examples described here, however, we have assumed that the rate of these extra processes is zero, so that $\mathrm{C}_{\mathrm{db}}=0$.

With a sudden complete reversal of the body-force acceleration (from $g=-1$ to $g=+1)$, the zone is expected to de-mix back to its original state with a sharp interface. As pointed out by Nikiforov (personal communication, 1994), single-point transport equations in a finite difference implementation have difficulty in calculating this process. We confirm 
that difficulty and have identified the problem. De-mixing is highly dissipative of energy. In contrast to mixing, in which clumps of a material do not collide with others of the same material, de-mixing is highly collisional within the field of each material. The inelastic collisions involve much more microphysics than our current model contains, in particular, splash with possible entrainment of the other material. Two-field models calculate de-mixing with deceptive ease in the circumstance of no splash (the "splat-and-stick" limit). When $\alpha_{1}$ or $\alpha_{2}$ has increased back to 1.0 , the calculation holds the volume fraction constant thereafter, and the incompressibility condition for that field ensures that the velocity comes at once to rest, which results in a continuous dissipation of collisional energy as each fluid returns to its pure state. This matter is discussed more extensively in Chapter 4 for the two-field calculations in this limiting mode of de-mix representation.

\subsection{Numerical results for a rapid transient}

We have not yet investigated the possible inelastic collisional interactions within a fluid and their appropriate dissipative representation. The issue of particular concern to our developments is the question of self-similarity during the rapid-transient phase of turn around induced by acceleration reversal. Figures 7.3.2 and 7.3.3 describe the computed behavior of our spectral equations during this process. The principle issue concerns the variations of $a(k)$. As shown in Fig. 7.3.2, the other variables, $b(k), R_{n n}(k)$, and $R_{y y}(k)$ have much smaller transient response. With the reversal of $\mathbf{g}$ there is likewise an instantaneous reversal of $\partial p / \partial y$. The differential acceleration driver to $a(k)$ thus reverses; for a brief period of adjustment that term works in concert with the drag terms towards reversal of the sign of $a(k)$; in this regard the entire spectrum of $b(k)$ becomes active. For the acceleration stage, the pressure and density gradient terms in Fig. 7.3.1(a) balance the drag terms at high wave numbers, which results in negligible changes in $a(k)$, vanishingly small $\partial a(k) / \partial t$. Immediately after the acceleration reversal at $t=65$, the pressure gradient term changes sign, which causes a relatively larger change of $a(k)$ for the high wave numbers. In this respect we 
say that the high wave number portion of the $b(k)$ spectrum has changed from passive to active.

When $a_{i}(k)$ reverses sign, the mass flux becomes counter-gradient to the density variation; the two driver terms in the evolution equation for $\mathrm{a}(\mathrm{k})$ now work in opposition to one other. To further illustrate this point, we see that the pressure gradient term and the density gradient term both counter the drag terms in Fig. 7.3.1(a), whereas in Fig. 7.3.1(b), immediately following the acceleration reversal, the density gradient term counters not only the two drag terms, but also the pressure term. (As pointed out by Youngs (personal communication, 1993), this opposition is necessary for describing his de-mixing experiments: differential acceleration works to compact the zone while density-gradient turbulence flux tends to preserve its dispersal.)

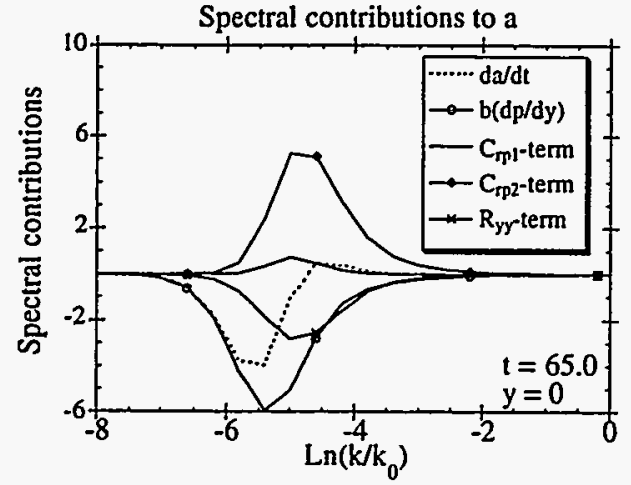

(a)

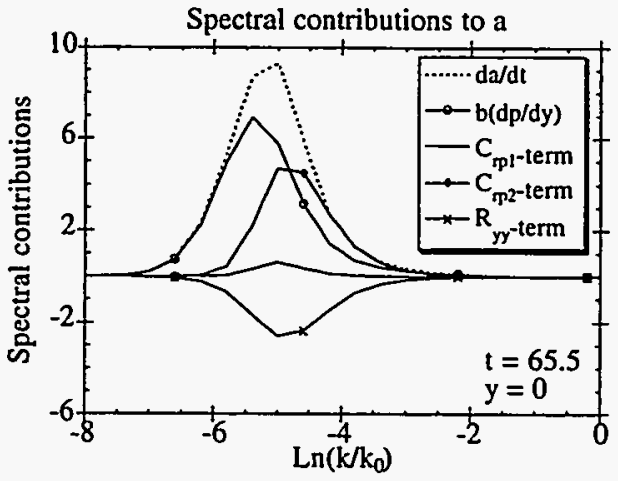

(b)

Figure 7.3.1. Spectral contributions of the dominant sources and sinks to the a equation for: (a) mixing $(g=-1)$, and $(b)$ demixing, immediately after acceleration reversal $(g=1)$.

Although the brief opposition of $\mathrm{a}(\mathrm{k})$ and $\partial \mathrm{p} / \partial \mathrm{y}$ during the slowdown parts of turnaround give a negative source to the components of $R_{i j}(k)$, the net effect is small. For the calculation of turnaround results, we used the spectrally blocked version of the base-case conditions described for Fig. 7.2.12, allowed the attainment of self-similar mixing, reversed $\mathrm{g}$ (from -1 to +1 ) at $t=65$, and observed the turnaround until its more-or-less completion at 
$t=80$. The transient phases of structure for $a(k)$ are shown in Fig. 7.3.2(a). There is a clear departure from the self-similarity during the process. The large scales (small wave numbers) exhibit a more immediate response than the small scales (at large wave numbers). A simple amplitude-modulated function with slowly shifting position in $\mathrm{k}$-space for its maximum is not strictly what occurs; although, in effect, single-point model equations assume this type of behavior.

Figure 7.3.3 shows that, although the de-mixing process that follows flow reversal cannot be self-similar, the spectral forms as shown in Fig. 7.3.3 return to almost precisely those that were present before the acceleration reversal. The implications are important for moment-integral derivations of single-point transport equations: almost-universal forms occur in much wider circumstances than just those that are self-similar.

Variations of spectrally integrated quantities across the TMZ are illustrated in Fig. 7.3.4. Figure 7.3.4(a) shows that the heavier clumps of fluid respond more quickly to gravity reversal than the light clumps. The most startling observation is in Fig. 7.3.4(b), which shows negative values of $\mathrm{b}$ where the heavier fluid is falling back into itself.

The anomaly is also seen in Fig. 7.3.5, in which the average density exceeds 2.0 at this fall-back locality. In the two-field calculations of Chapter 4, this impossibility is avoided by the dissipative technique described above for one possible type of mixing. The same "stick-and-splat" fix technique could also be employed here, but the topic requires extensive consideration and discussion for more general de-mixing circumstances.

The base case calculation without spectral blockage was also performed for the mixing of two fluids with a larger density ratio, $\rho_{2} / \rho_{1}=5.0$. The results exhibit essentially the same attainment of self-similarity, with spectral exponents corresponding to those in Fig. 7.2.2. 


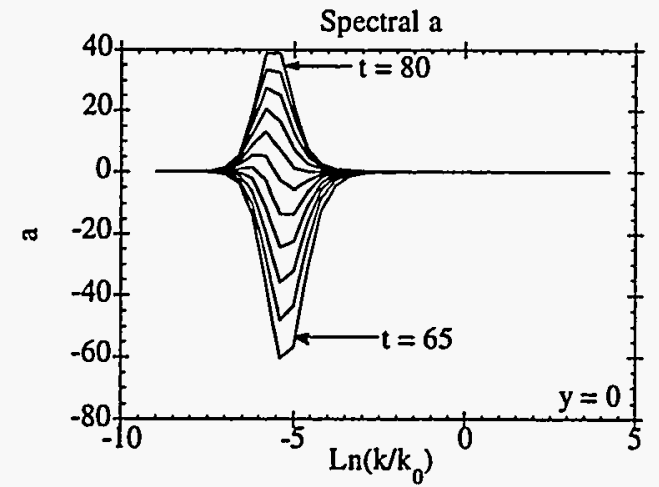

(a)

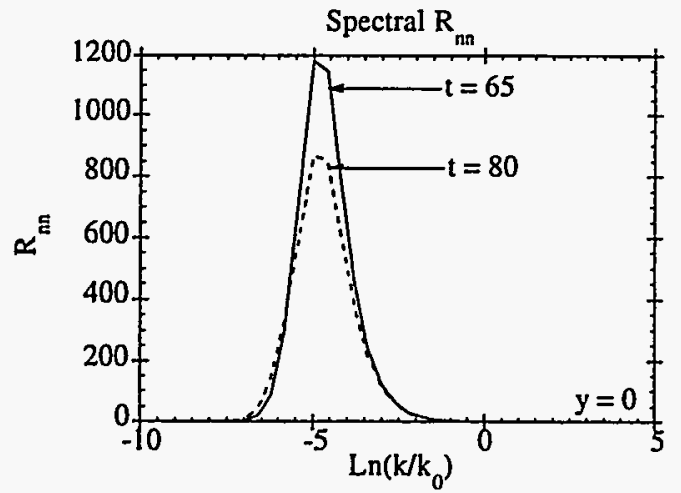

(c)

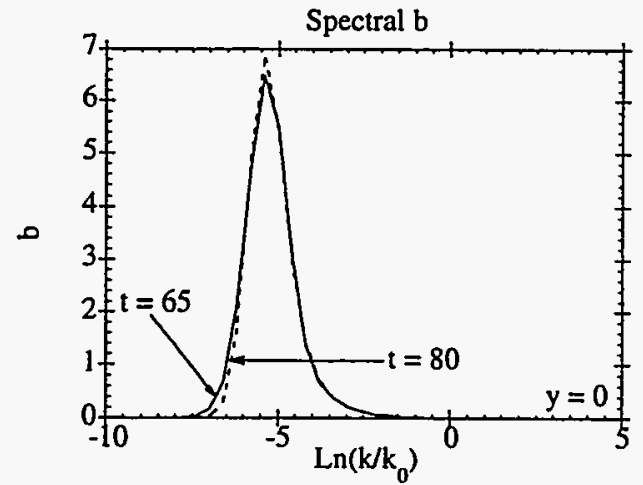

(b)

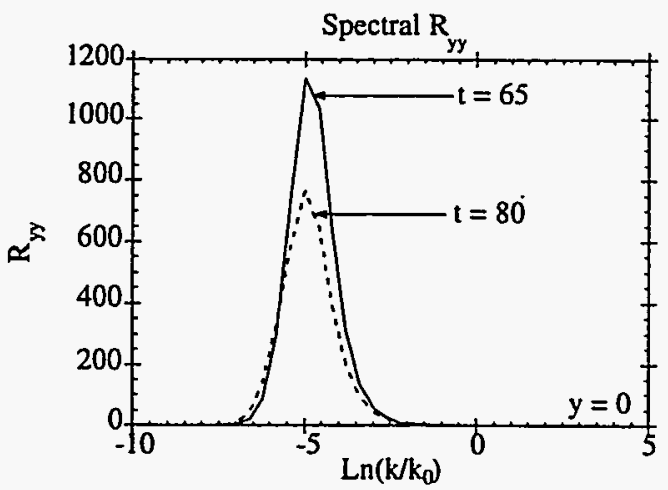

(d)

Figure 7.3.2. Spectra at times $t=65$ and 80 , at the position of the initial fluid interface, for: (a) the net massflux velocity, a (including plots for every $\Delta t=1.5$ ), (b) the specific volume-density correlation, $b,(c)$ the contraction of the Reynolds stress tensor, $R_{n n}$, and (d) the $R_{y y}$ component of the Reynolds stress tensor. 


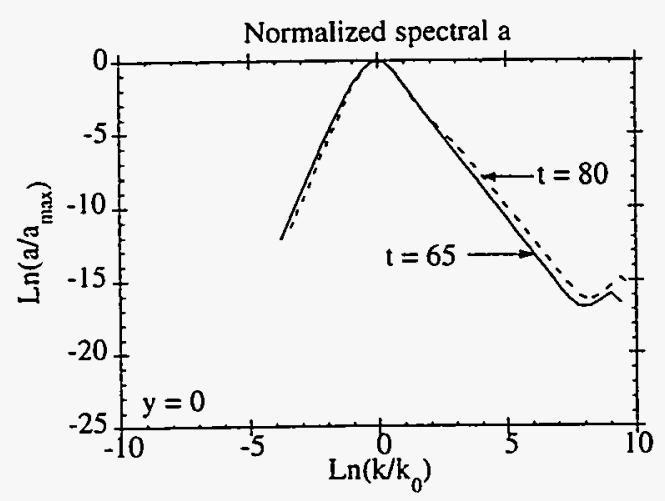

(a)

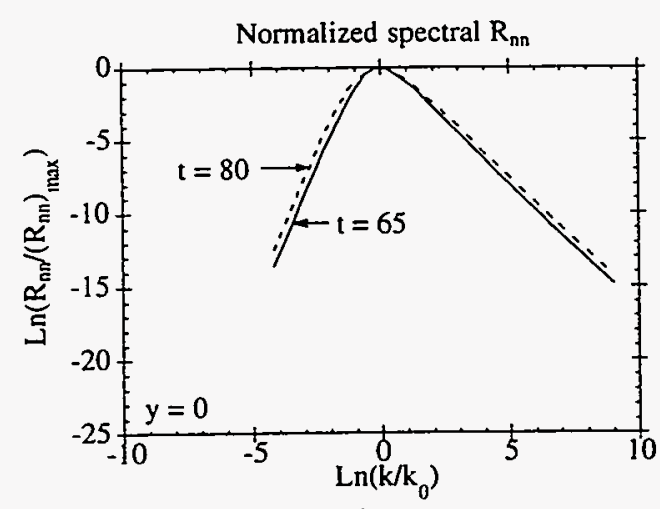

(c)

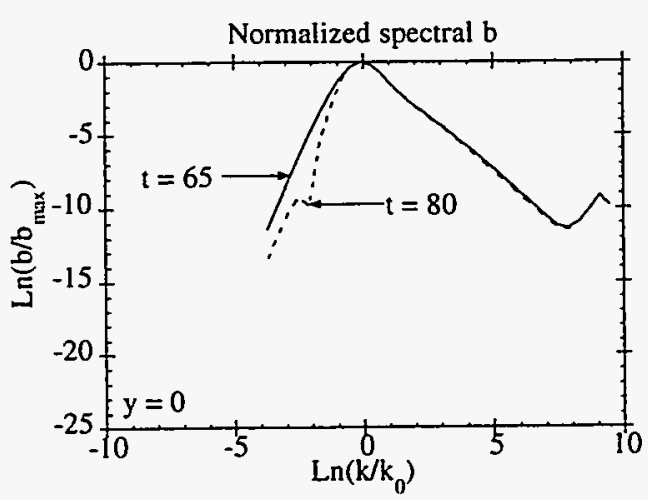

(b)

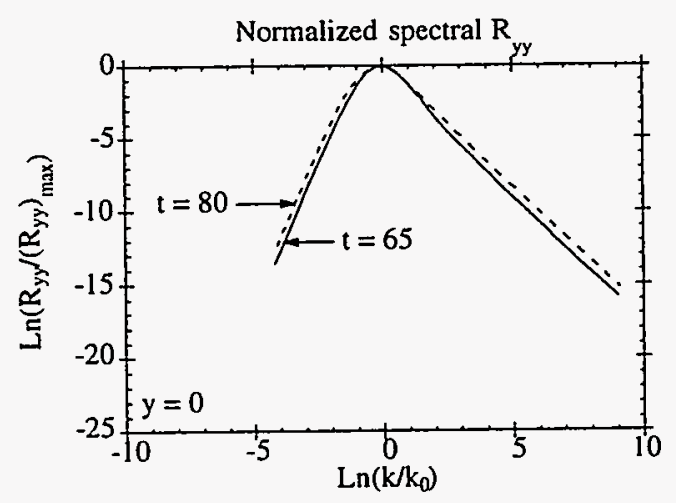

(d)

Figure 7.3.3. Normalized spectra for $t=65$ and 80 , at the position of the initial fluid interface, for: (a) the net mass-flux velocity, a, (b) the specific volume-density correlation, b, (c) the contraction of the Reynolds stress tensor, $R_{n n}$, and, (d) the $R_{y y}$ component of the Reynolds stress tensor. 


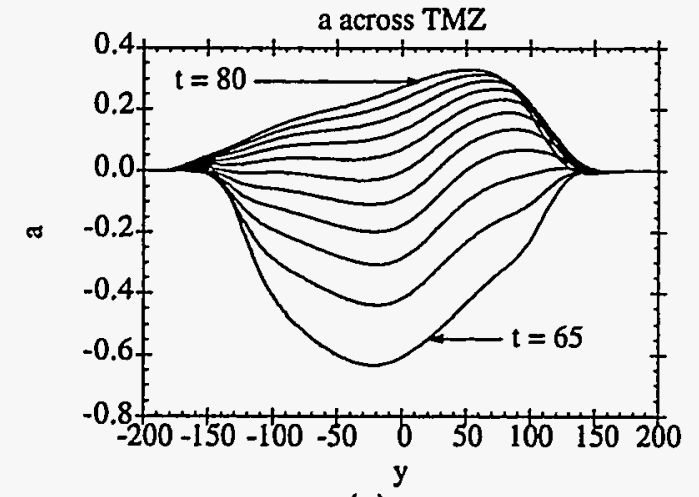

(a)

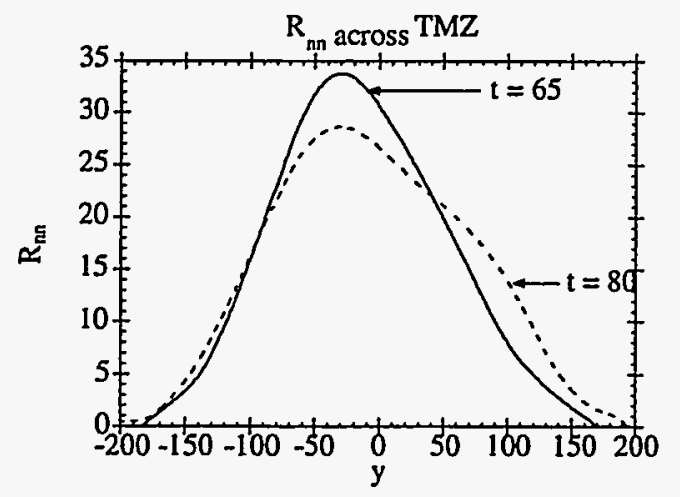

(c)

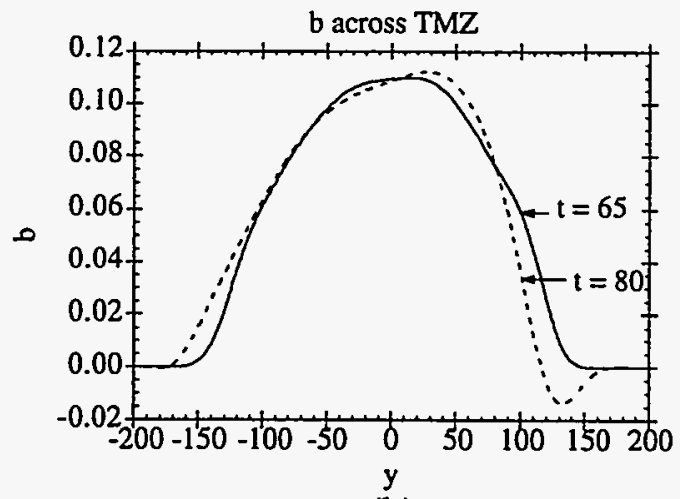

(b)

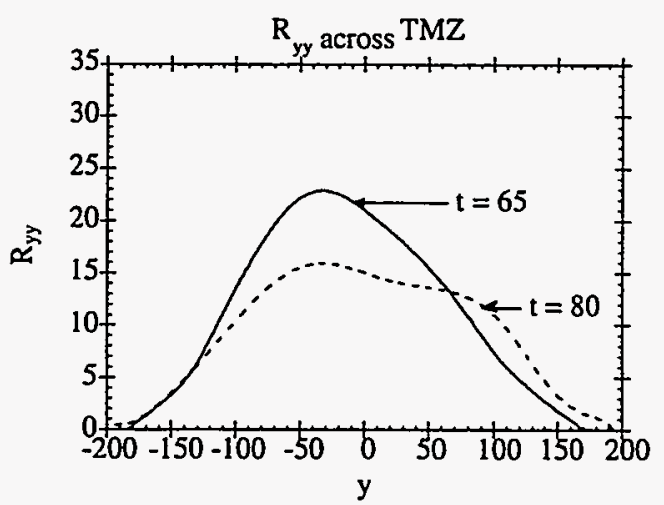

(d)

Figure 7.3.4. Profiles of spectrally integrated variables across the TMZ for $t=65$ and 80 of (a) the net massflux velocity, a (including plots for every $\Delta t=1.5$ ), (b) the specific volume-density correlation, $b,(c)$ the contraction of the Reynolds stress tensor, $R_{n n}$, and (d) the $R_{y y}$ component of the Reynolds stress tensor.

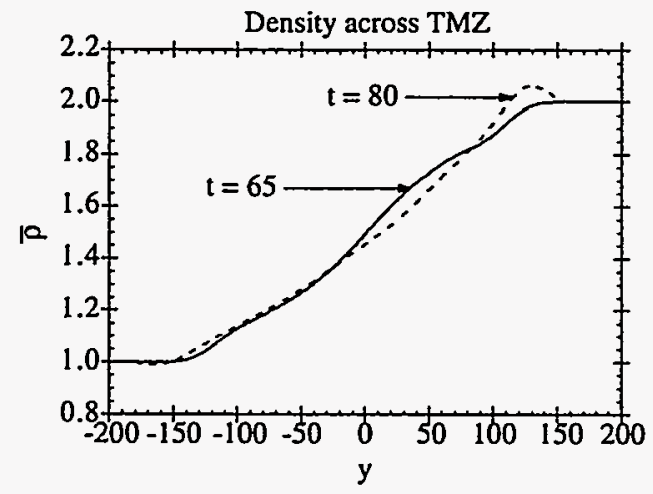

Figure 7.3.5. Profiles of density across the TMZ for $\mathrm{t}=65$ and 80 . 


\begin{tabular}{|c|c|c|}
\hline Quantity & $\begin{array}{c}\text { Low-k } \\
\text { exponent }\end{array}$ & $\begin{array}{c}\text { High-k } \\
\text { exponent }\end{array}$ \\
\hline $\mathrm{R}_{\mathrm{ij}}(\mathrm{k})$ & 3.99 & -1.74 \\
\hline $\mathrm{b}(\mathrm{k})$ & 3.87 & -1.71 \\
\hline $\mathrm{a}_{\mathrm{i}}(\mathrm{k})$ & 3.92 & -2.37 \\
\hline
\end{tabular}

Table 7.3.1. Power law behavior for both the low and high wave numbers for the transported spectral variables for the density ratio of 5.0 .

For the case with a density ratio of 5.0, as in Fig. 7.2.3, the ordering of exponent values in Table 7.3.1 for low wave numbers is the same and for the same reasons having to do with the effects of $\mathrm{C}_{\mathrm{fb}}$. For high wave numbers we see again the large contrast between the cascade-dominated behavior of $R(k)$ and $b(k)$, for which $n \approx-5 / 3$ and the drag-dominated behavior of $\mathrm{a}(\mathrm{k})$, for which $\mathrm{n} \approx-7 / 3$. The value of $\alpha$, the self-similar growth coefficient for $h_{1}$, is 0.11 , which is appreciably larger than either the base-case result or the experimentally observed result. In addition, $\mathrm{h}_{2} / \mathrm{h}_{1}$ approaches the magnitude 1.08 , which is low.

\subsection{Local vs. nonlocal source term}

Two intriguing disagreements with experiments are especially noticeable in the results discussed so far (see Table 7.4.1). One is the coefficient for self-similar growth of $h_{1}$, which is somewhat too large, and the other is the asymptotic ratio of $h_{2} / h_{1}$, which is too small. An intriguing clue to remediation of both discrepancies lies in the results described by Figs. 7.4.1-7.4.5. In those figures we compare the base case with local and nonlocal source terms for $R_{n n}(k)$ and $R_{y y}(k)$. Figure 7.4.1 shows the large contrast in results. With the local source, density exhibits sharp variations on each side of the TMZ; these are more spread out with the nonlocal source. 
The coefficient, $\alpha$, is sharply reduced to the value $\alpha=.042$, while the ratio of penetration depths, $h_{2} / h_{1}$, approaches the much larger value of 1.79 . In both respects the experimental values lie between the extremes.

\begin{tabular}{|l|c|c|}
\hline Source to $\mathrm{R}_{\mathrm{ii}}$ & $\alpha$ & $\mathrm{h}_{2} / \mathrm{h}_{1}$ \\
\hline local & .042 & 1.79 \\
\hline nonlocal & .079 & 1.05 \\
\hline experiments & $\sim .065$ & $\sim 1.30$ \\
\hline
\end{tabular}

Table 7.4.1. Self-similar coefficient, $\alpha$, and the asymmetry of the TMZ for the two numerical runs and experiments.

It thus appears that a more restrictive nonlocal formulation for $\mathrm{Q}\left(\mathrm{y}^{\prime}, \mathrm{y}\right)$ could produce closer agreement with experiments in both respects. The task of deriving such a formulation remains a challenge yet to be addressed.

The spectral behaviors are compared at low and high wave numbers in Figs. 7.4.2 and 7.4.3. At the low wave numbers, there is a significant effect that is discussed in Section 6.3. At the high wave numbers there is very little difference between the local and nonlocal formulations. Here we merely observe that the spectrally preserving local source is structurally dominated by the $\mathrm{C}_{\mathrm{fb}}$ effects, so that the exponent is lower than with the nonlocal source, which is not spectrally preserving and indeed tends to increase the exponent at low wave numbers. This complex set of interactions is discussed in more detail in Section 6.3.

Figure 7.4.4 is comparable to Fig. 7.2.4, with both showing the evolution towards self-similarity at a distance of 50 units below the original interface. The results are quite similar; in both cases the first influence to arrive is that of $a(k)$, which tends to impart $k^{-7 / 3}$ behavior at the high wave numbers, soon to be dominated, however, by the effects of cascade that convert the spectrum to $\mathrm{k}^{-5 / 3}$. At the lower wave numbers the spectrum arrives in completely self-similar 


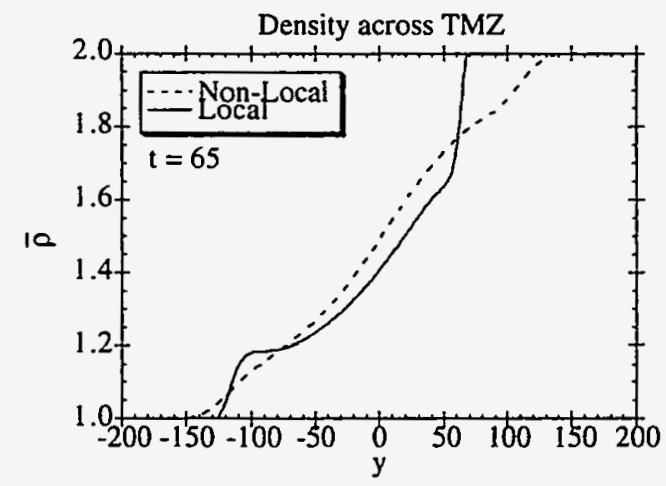

Figure 7.4.1. Comparison of the density across the $T M Z$ for a local source to $R_{i j}$ and a nonlocal source to $R_{\mathrm{ij}}$.

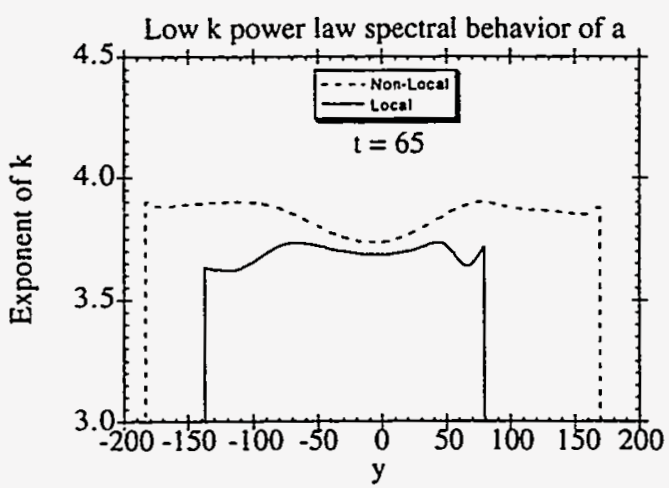

(a)

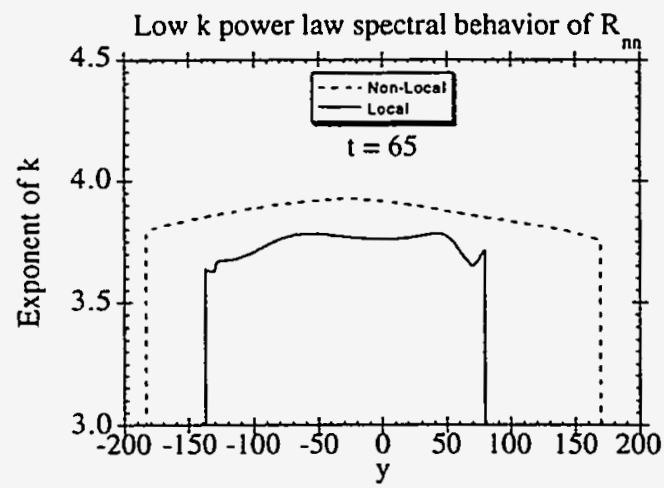

(c)

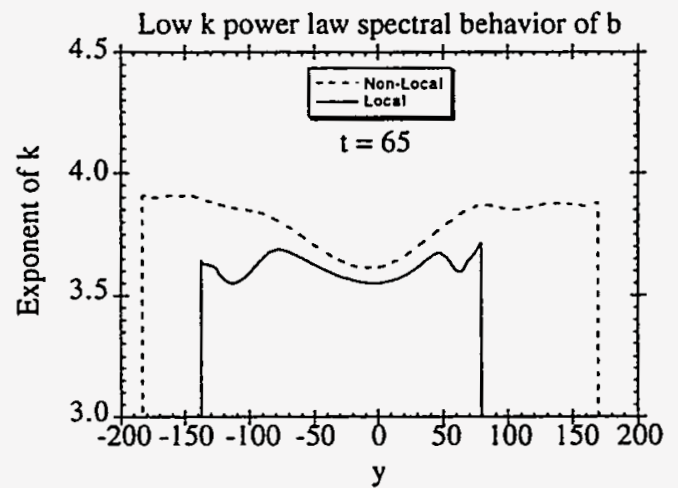

(b)

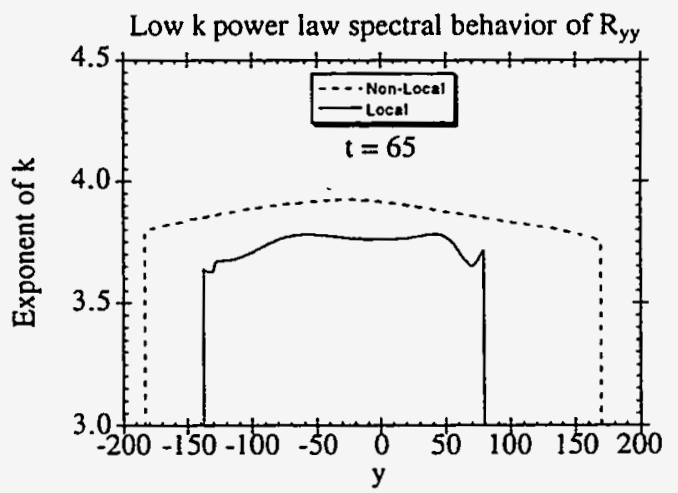

(d)

Figure 7.4.2. Comparison of the low wave number power law behavior of $k$ at $t=65$ for a local and nonlocal run for: (a) the net mass-flux velocity, a, (b) the specific volume-density correlation, b, (c) the contraction of the Reynolds stress tensor, $R_{n n}$, and, (d) the $R_{y y}$ component of the Reynolds stress tensor.

form. The contrast between local and nonlocal lateral propagation is manifested in slightly less departure from self-similarity at high wave numbers in the local case, especially in the 
spectra of $R_{n n}$ and $R_{y y}$. The reason is that nonlocal propagation favors more rapid lateral distribution of the larger scales, with corresponding slower lateral distribution of the smaller scales.

This difference in lateral propagation is especially apparent in Fig. 7.4.5, which shows the spatial distributions of scale lengths (reciprocals of wave numbers for the spectral maxima) at a late time in self-similar TMZ growth. Disregarding structural details in the profiles, we notice a significant difference between the local and nonlocal results. For the local source term, the length scales all tend to decrease towards the edges of the TMZ. With a nonlocal source term,

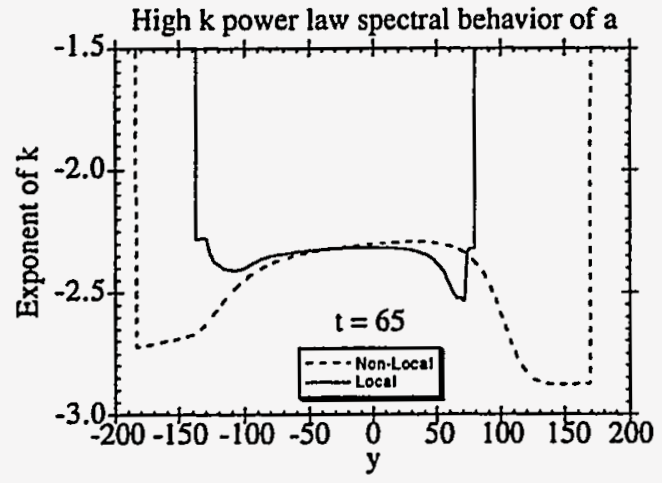

(a)

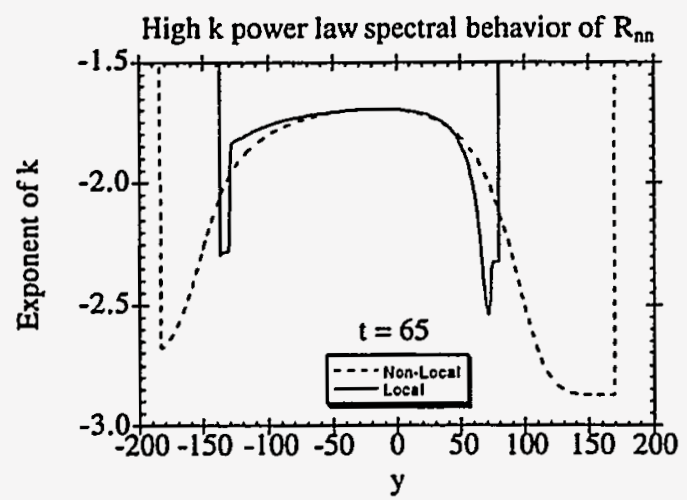

(c)

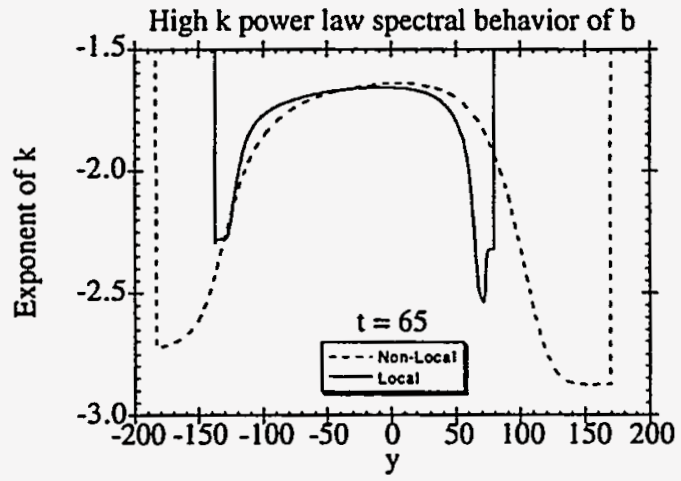

(b)

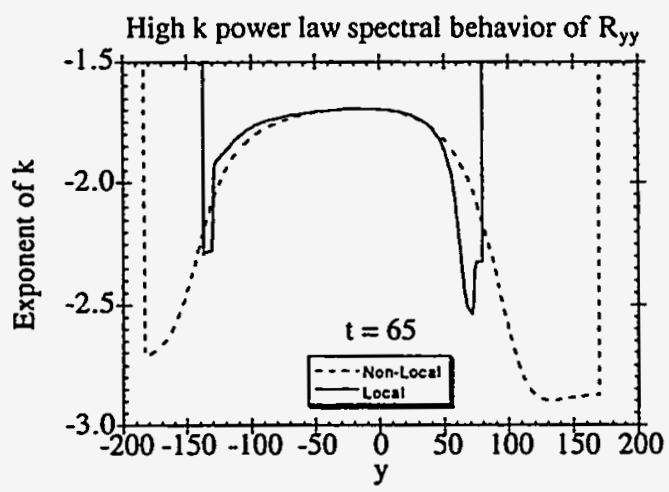

(d)

Figure 7.4.3. Comparison of the high wave number power law behavior of $k$ at $t=65$ for a local and nonlocal run for: (a) the net mass-flux velocity, $a$, (b) the specific volume-density correlation, $b$, (c) the contraction of the Reynolds stress tensor, $R_{n n}$, and, (d) the $R_{y y}$ component of the Reynolds stress tensor. 
the profiles for $a$ and $b$ are essentially the same, whereas for $R_{n n}$ and $R_{y y}$ the scales increase significantly towards the edges. It is this feature that especially demonstrates the desired consistency with Rayleigh-Taylor theory, as discussed in Section 6.2.

The issue of $\bar{\rho}$ variations across the TMZ can be put into clearer perspective by further consideration of the experimental observations. Figure 7.4.6 is adapted from the Russian report by Kucherenko, et al. (1991) in which they give a very thoughtful analysis of the situation for various different density ratios, $n=\rho_{2} / \rho_{1}$. The principal issue concerns the identification of edge locations for the TMZ for which they use both photographic and x-ray techniques. They define edge positions in terms of a fractional quantity, $\delta$, which designates fraction of purity for the heavier fluid at which the edge is defined to be located. Typical values are $\delta=0.10,0.90$ and

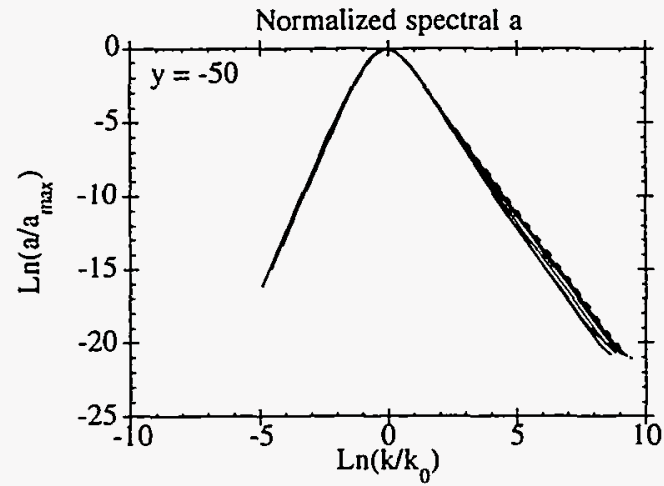

(a)

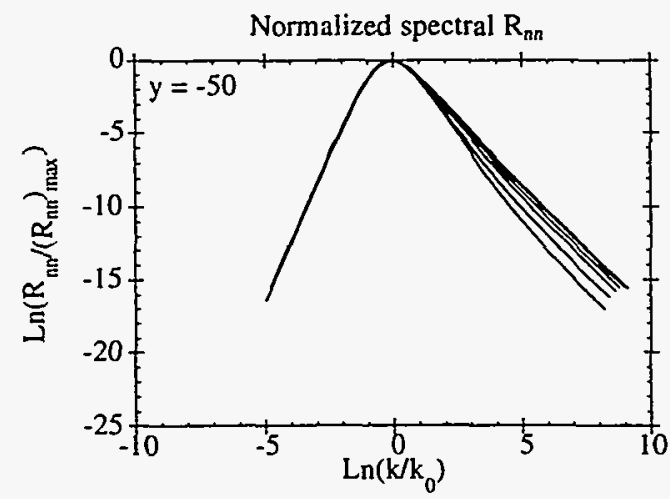

(c)

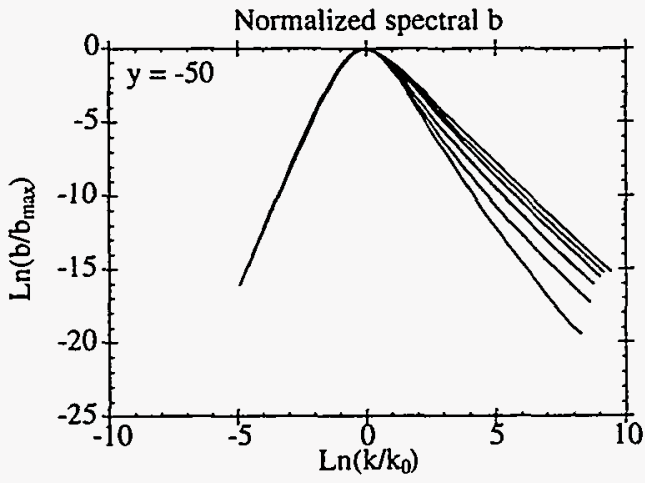

(b)

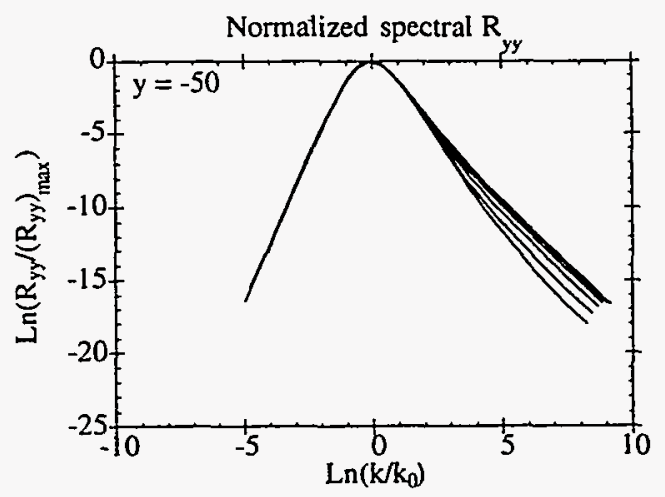

(d)

Figure 7.4.4. Normalized spectra for $t=30,35,40,45,50,55,60$, and 65 , a distance of 50 units of length below the centerline of the TMZ for: (a) the net mass-flux velocity, a, (b) the specific volume-density correlation, $b$, (c) the contraction of the Reynolds stress tensor, $R_{n n}$, and, (d) the $R_{y y}$ component of the Reynolds stress tensor. 
$\delta=0.01,0.99$ (this last being stated as 0.98 on one of the figures in their report, presumably erroneously). Figure 7.4.6(a) shows the variation of $\bar{\rho}$ across the TMZ for a typical example. Error bars indicate the uncertainty, especially with implications for edge location. With $\delta=0.01,0.99$, the distances $h_{1}$, and especially $h_{2}$ depend strongly on how the datum points are connected through the envelope of error bars. With $\delta=0.10,0.90$, the sensitivity is reduced and the ratio, $h_{2} / h_{1}$, is considerably smaller. Figures $7.4 .6(b)$ and $7.4 .6(c)$ illustrate the effects of choosing different edge criteria. Combining both Russian and British data, we see in Fig. 7.4.6(d) a considerable scatter in data. The figure also shows the base-case numerical results for both the local and nonlocal sources. Refer to Table 7.4.1 for the values of $h_{2} / h_{1}$. The calculation with the nonlocal source is considerably closer to the most-likely experimental value, but it is apparent that more precise data would be useful as a basis for validation of any modified form for $\mathrm{Q}\left(\mathrm{y}^{\prime}, \mathrm{y}\right)$ in the expression for nonlocal creation.

A calculation was performed for a somewhat different departure from the base case to examine the consequences of modifying the low-wave-number initialization of $b(k)$. In the initial-condition formula for $\mathrm{b}(\mathrm{k})$ in the interface computational cell,

$$
\mathrm{b}(\mathrm{k})=\frac{\gamma_{1} \mathrm{k}^{\mathrm{n}}}{1+\gamma_{2} \mathrm{k}^{\mathrm{n}+5 / 3}},
$$

we used $n=4$ in the base case and $n=2$ in this modified run. Here $\gamma_{1}$ and $\gamma_{2}$ are the same as in the beginning of this section. All other conditions were the same for the two calculations. The purpose for the comparison is to explore the possibility of nonuniqueness for late-time self-similarity as induced by the persistence of structure at low wave numbers. This intriguing question has been discussed at length by previous investigators. If $n \geq 4$ it is thought that a single self-similar form function will emerge (Batchelor, 1986); for $\mathrm{n}<4$ there is reason to believe that the form function will persistently depend on the value of $n$. In three-dimensional $\mathbf{k}$-space the continued influence of low-wave-number structure on selfsimilarity gives a rich set of potential form functions. In the current comparison we see that 
with $\mathrm{n}=2$ the differences from the base-case results are so slight, largely due to choice of modeling, that we could not effectively illustrate them in figures. With $n=2$

- the values of $\mathrm{a}(\mathrm{k}), \mathrm{R}(\mathrm{k})$, and $\mathrm{R}_{\mathrm{yy}}(\mathrm{k})$ are slightly larger,

- $\mathrm{h}_{2} / \mathrm{h}_{1}$ is slightly smaller (asymptotically, $\mathrm{h}_{2} / \mathrm{h}_{1} \approx 1.03$ ),

- the TMZ spreads a bit faster, $\alpha=0.098$,

- the spectra all propagate slightly faster toward low wave numbers.

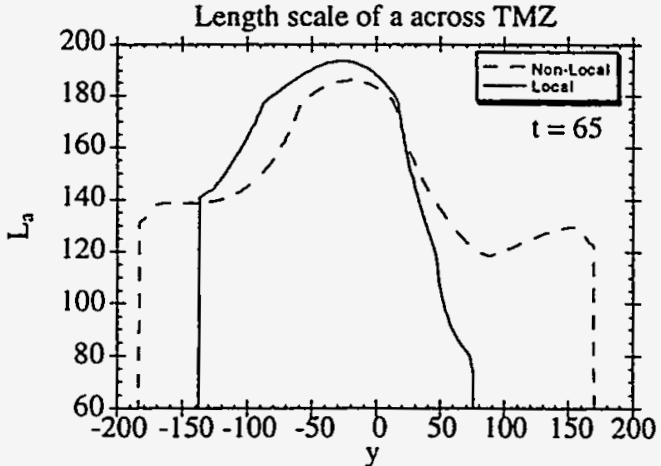

(a)

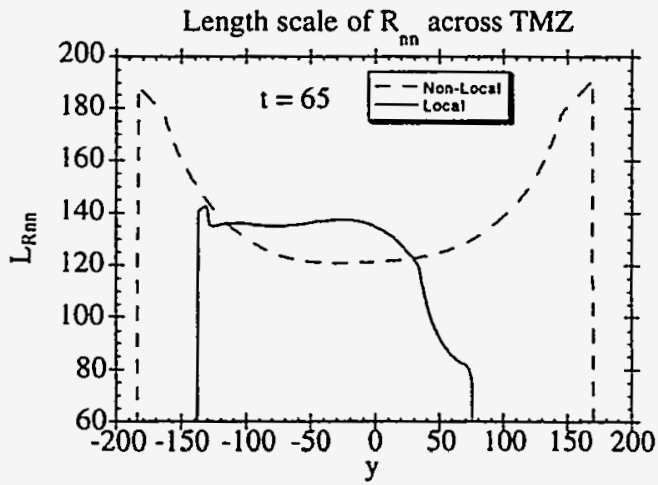

(c)

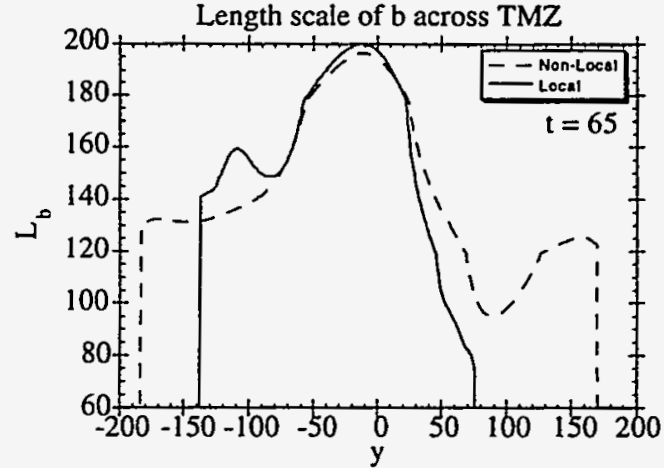

(b)

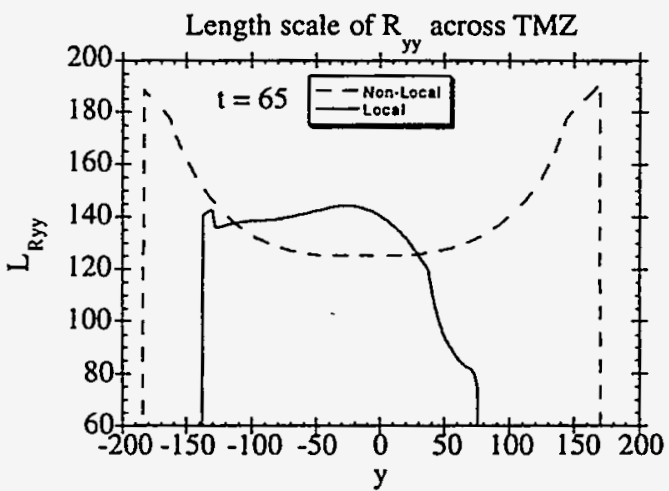

(d)

Figure 7.4.5. Comparison of the profiles of the length scales across the TMZ for a local and nonlocal run at $\mathrm{t}=65$ for: (a) the net mass-flux velocity, a, (b) the specific volume-density correlation, $b,(c)$ the contraction of the Reynolds stress tensor, $R_{n n}$, and, (d) the $R_{y y}$ component of the Reynolds stress tensor.

These trends are qualitatively consistent with the fact that $n=2$ gives larger $b(k)$ values for small wave numbers, which persistently causes creation enhancement for $a(k)$ and in turn the Reynolds-stress components. 


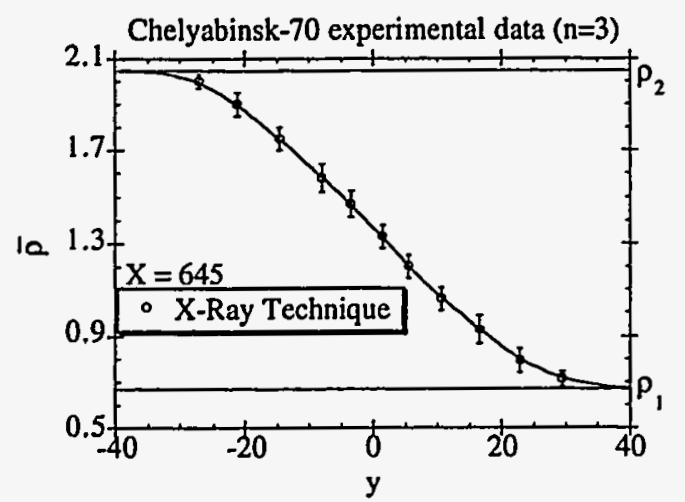

(a)

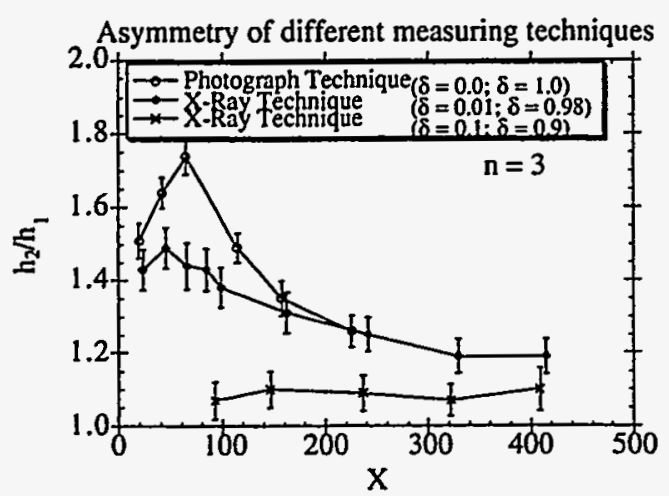

(c)

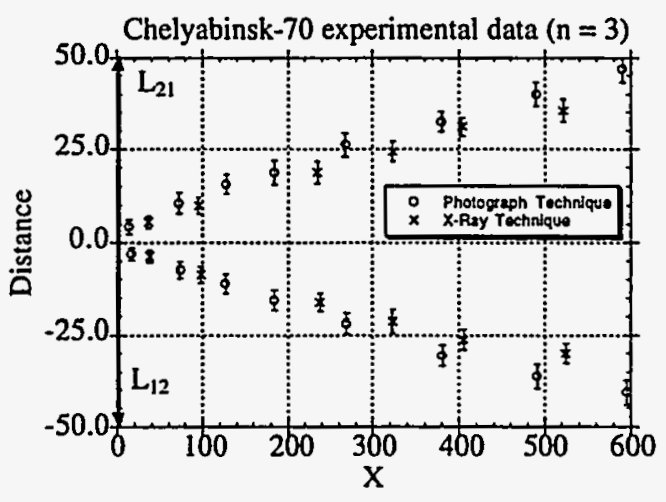

(b)

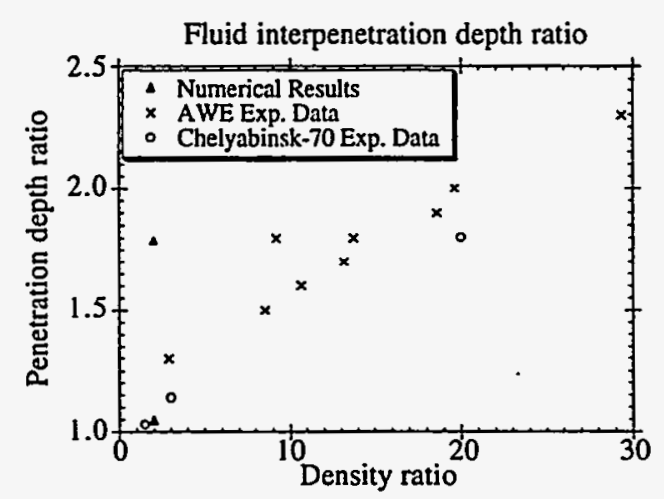

(d)

Figure 7.4.6. Experimental data from Chelyabinsk-70 (Kucherenko et al. 1991) for: (a) concentration profile through TMZ, (b) bubble and spike penetration depth, (c) asymmetry of spike to bubble ratio, and (d) comparison of numerical results to experiments (Kucherenko et al. 1991; Smeeton and Youngs, 1987).

As a last calculational example, we discuss the effects that result from a change in $\mathrm{C}_{\mathrm{fb}}$, the "bubble-doubling" coefficient. In the base case, $\mathrm{C}_{\mathrm{fb}}=0.50$; in the comparison calculation $\mathrm{C}_{\mathrm{fb}}$ is increased to 0.65 . The most prominent of the results are shown in Fig. 7.4.7. As discussed in Section 6.3, there are competing processes at low wave numbers, for which the three most prominent contributors are

- the initialization exponent, $\mathrm{n}$, for $\mathrm{b}(\mathrm{k})$,

- the value of $\mathrm{C}_{\mathrm{fb}}$,

- the nonlocal source for Reynolds stress.

The first of the above three bullets dominates the low wave number spectral behavior. A slightly modified value of $\mathrm{n}$ determines the exponent for all the variables which then persists into the self-similar stages. Nonlocality of source to Reynolds stress tends to 
increase the exponent; only the local source is spectral-form preserving (i.e., imparts the lowwave-numbers form of $\mathrm{a}(\mathrm{k})$ without distortion). The $\mathrm{C}_{\mathrm{fb}}$ contribution tends to decrease the exponent at low wave numbers for $b(k)$, with the effect spreading to $a(k)$ and thence to $R_{n n}(k)$ and $R_{y y}(k)$. The net effect of these influences is seen in Fig. 7.4.7, which shows the greatest change in $b(k)$, less in $a(k)$, and even less in the Reynolds-stress spectra. Also apparent in Fig. 7.4.7 is the greater width of the TMZ for the calculation with larger $\mathrm{C}_{\mathrm{fb}}$. At high wave numbers the effects of this increase in $\mathrm{C}_{\mathrm{fb}}$ are negligible. Comparisons of selfsimilar spectral forms are not illustrated in the figures; the forms are almost precisely the same as for the base case; with $\mathrm{C}_{\mathrm{fb}}=0.65$ all of the normalized spectral forms show very slightly magnitudes at low wave numbers and slightly lower at high wave numbers.

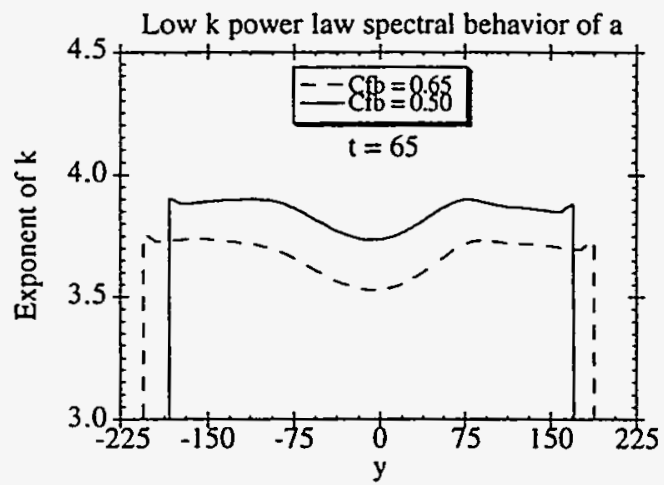

(a)

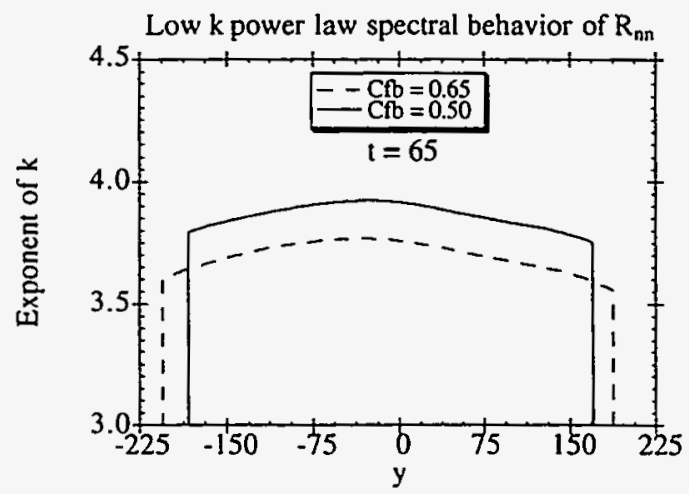

(c)

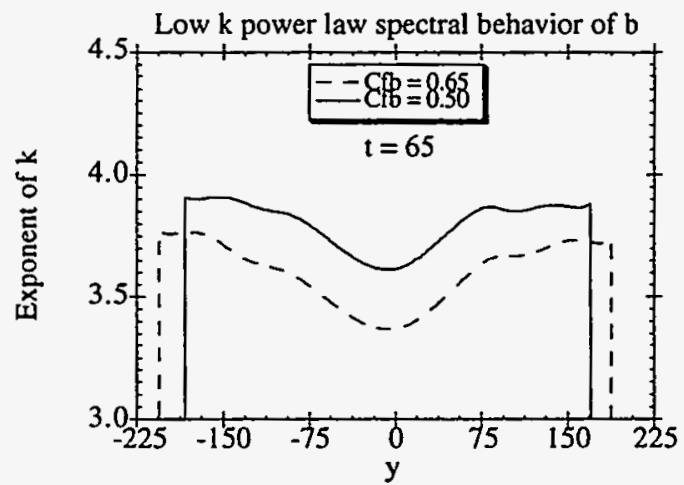

(b)

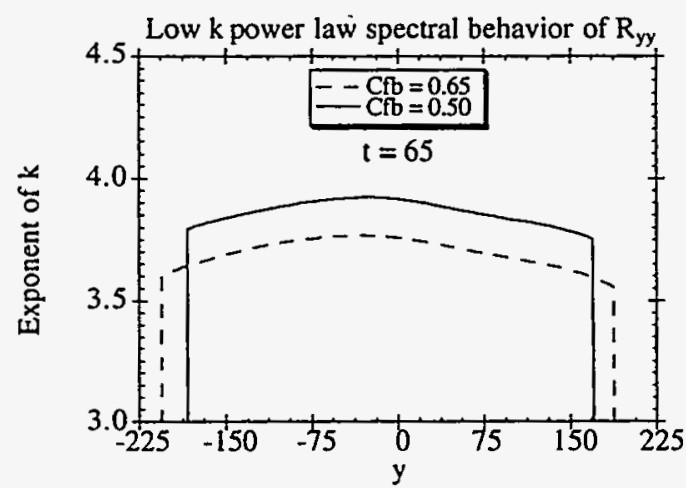

(d)

Figure 7.4.7. Comparison of the low wave number power law behavior of $k$ at $t=65$ for two different values of $C_{f b}$ for: (a) the net mass-flux velocity, a, (b) the specific volume-density correlation, $b$, (c) the contraction of the Reynolds stress tensor, $R_{n n}$, and, (d) the $R_{y y}$ component of the Reynolds stress tensor. 


\subsection{Summary}

In this chapter we have presented a base case to examine the behavior of our model for constant acceleration. We have shown reasonable agreement with experimental data. In addition to the comparisons to experiment, we have also demonstrated the spectral behavior of the model by showing: (1) the self-similar development for the case of a constant acceleration, (2) the deviation from self-similarity and the eventual return to self-similarity for the case of a rapid transient, and (3) the low and high wave number spectral behavior.

Results are presented to contrast the spectral behavior for a local source to the Reynolds stress equation with a nonlocal source to the Reynolds stress equation. These results along with the results for two different values of the constant $C_{f b}$ in the $b(k)$ transport equation are given to demonstrate the influence of different facets of our model.

The spectral self-similar results given in this chapter represent the first such results for a variable density mixing flow. It has been observed many times experimentally that the Rayleigh-Taylor instability eventually becomes self-similar. Many single-point K- $\varepsilon$ type models have captured this physical-space self-similarity, but to this day no one has demonstrated spectral self-similarity. The departures from self-similarity as demonstrated in the results for the rapid transient demonstrate the value of the spectral model. It is this feature that the single-point equations are incapable of capturing. 


\section{Summary}

\subsection{Conclusions}

A spectral turbulence transport model for variable density flows has been formulated and applied to a self-similar turbulent mixing layer. Comparisons with experiment indicate that the model developed herein is capable of capturing the evolution of the self-similar stage of mixing that ensues from the Rayleigh-Taylor instability. In addition to matching the available experimental data on Rayleigh-Taylor instabilities, the formulation provides much more meaningful physical insight into the structure of the turbulence beyond that which single-point models are capable of providing. Examples are also given for a case of acceleration reversal to demonstrate the spectral behavior throughout a rapid transient in the mean flow driver.

The exact unclosed two-point transport equations for the primary mass-averaged variables of this spectral model are derived directly from the Navier-Stokes equations. The transport equations have been Fourier transformed with respect to the separation vector between the two points. Due to their extreme complexity, the transport equations are angularly integrated in $\mathbf{k}$-space to reduce the transport models to a simpler form in scalar-kspace. This reduction is justified by the fact that as a step to fully three-dimensional $\mathbf{k}$-space, $\mathrm{k}$-space captures the essence of the physics and is much less computer intensive. These derivations are given by Clark and Spitz (1995). Constraints and guidelines have been identified and incorporated for modeling the necessary closures for this variable density inhomogeneous model in k-space. Such things as tensorial and dimensional correctness, Galilean invariance, and conservation, act as constraints to abide by while appealing to simplicity to construct the closures for this model.

This spectral formulation represents the first attempt at examining variable density inhomogeneous turbulence from a two-point approach. Admittedly, much of the spectral information associated with the physics is lost in the angular integration of the transport 
equations, but we argue that this k-space statistical model serves two useful purposes: (1) it provides a decent foundation upon which to base extensions of complexity for future twopoint modeling, and (2) it provides an extension to our current understanding of the physical processes involved with turbulent mixing. Thus, this spectral model allows us to examine the foundations of complex fluid flow, i.e., understand how to pick out the essence of the main driving features of a complicated flow and to understand more fully complicated coherent structures of turbulent flows.

Two chapters of this dissertation are dedicated to single-point modeling. Chapter 3 reviews the BHR turbulence transport model and Chapter 4 reviews a generic two-fluid approach. Both approaches are applied to an inhomogeneous variable density mixing layer. These two chapters summarize the background work that was done in preparation for the spectral part of this research. Our objective for the single-point work was to show the current state of variable density turbulent modeling and that indeed these single-point models can be calibrated to match experiment, but on the other hand, each of these two approaches has its major deficiencies. We feel that the identification of these deficiencies provide a decent motivation to formulate the spectral model of Chapter 5 , which purports to decrease the number of assumptions (relative to single-point models) that must be made and represents much more of the physics than either of the single-point approaches. Two major enhancements of the spectral model over any single-point model are: (1) the alleviation of the need for a transport equation for the length scales of the transported turbulence variables, and (2) the alleviation of the assumption of spectral equilibrium (a requirement for the validity of the single-point equations). The second of these two enhancements enables a spectral model to describe transient flows that are out of spectral equilibrium, whereas a single-point model cannot. Thus, with a spectral model, we are able to correlate the concept of turbulence decay with the cascade of energy from large turbulent scales down to smaller scales defined by a transfer rate that may vary with time. 
The spectral model also allows us to develop a systematic way to incorporate increasingly more sophistication into a single-point model through spectral integration. As a result, we are able to develop a single-point model that includes more physics than the current single-point models. This technique gives us a basis for determining when a singlepoint model is adequate to describe a flow, i.e., the flow is in spectral equilibrium, and when we are forced to resort to the more expensive spectral model, i.e., the flow is out of spectral equilibrium due to rapid transients in the mean flow drivers. When the flow is in spectral equilibrium, the length scales maintain a constant ratio, and the single-point models are adequate for describing the flow, but when the flow experiences a transient, the length scales fluctuate relative to one another and a full spectral model is needed to describe the flow until it has once again returned to a self-similar state. A spectral model allows us to investigate this rate of return to self-similarity and identify the primary competing processes responsible for these rates, such as mean flow time scales versus cascade and decay time scales.

This spectral model has enabled us to identify spectral self-similarity in the mixing of two fluids for the first time. It has been known for some time, both experimentally and numerically, that the turbulent mixing due to the late stages of the Rayleigh-Taylor instability is a self-similar process. This model has, for the first time, allowed us to examine the spectral relations between the various components of the flow and gain insight into the realm of validity for the single-point models. We have been able to identify independent selfsimilar behavior for both k-space and physical space. That is, we observe that as we transverse the TMZ in physical space we see little change in the form of the self-similar spectra in k-space. This is an extension of what one sees in the constant density free shear layer as reported by Besnard et al. (1990) in their BHRZ report. This same insight can now be applied to other such self-similar flows such as the mixing of a free shear layer due to a Kelvin-Helmholtz instability. With this variable density model, an extension to buoyancy driven Kelvin-Helmholtz instability can be studied (Snider \& Andrews, 1994). 
A spectral model for inhomogeneous variable density turbulence has been pondered for some time, but has never been brought to fruition. This work now represents a culmination of ideas from past researchers as well as our own contributions to produce the first working spectral model. To complement the previous research in spectral turbulence transport modeling, this research has made three significant contributions: (1) a nonlocal source term for the Reynolds stress transport equation that accounts for the global pressure effects due to incompressibility, (2) a source term to the $b(k)$ equation driven by the densitygradient that contributes to the "bubble doubling" phenomena of turbulent structures as observed by experiment, and (3) a configurational source term to the $b(k)$ equation that is rigorously derived for the two-fluid circumstance. All three of these contributions resulted in significant improvements to the spectral model.

This spectral model provides the physical foundations, above and beyond the singlepoint transport models, for the accurate modeling of flows that are aligned with real life situations. For example, the modeling of a flow through a pipe that experiences many turns and is thus never self-similar would be impossible with a single-point transport model. However, the spectral model developed herein allows for the description of these types of flows, and, hence, we can see the value of a spectral model for industrial applications.

\subsection{Future research directions}

The development of this spectral model forges the way for many different directions of future research. With the explicit presence of viscosity in the spectral model, future investigations can easily include the study of critical Reynolds numbers necessary for the transition to turbulence. This is not as easy with the single-point model due to the fact that the viscous dissipation term must be modeled by the formulation of an ad hoc dissipation rate equation.

This model is developed for scalar-k-space. The next obvious extension is to a model for fully three dimensional $k$-space. This will allow for the identification of coherent 
structures associated with anisotropy's of a flow. The identification of the relationship between anisotropies in $\mathbf{k}$-space and anisotropies in physical space will complement this study immensely.

Many industrial flows of interest to the turbulence community involve chemistry. A very useful direction will be to couple this spectral model with preexisting software packages that contain chemistry. The effects of exothermic and endothermic reactions in a flow can be coupled to the concept of the $b(k)$ in this model. The density variations associated with a fluctuating temperature can be coupled to the spectral model.

As the model currently exists, there is only one nonlocal source term due to the pressure effects in the Reynolds stress transport equations. Nonlocal effects can also be incorporated to account for coupling to the mean flow shear. This term was kept as a local term in this study due to the fact that creation of Reynolds stress via mean flow shear in the Rayleigh-Taylor instability is negligible compared to the other source terms. The extension of this term to its nonlocal representation will enhance this model's capabilities when examining other types of flows where shearing has a greater influence.

One issue that was never fully resolved in this study is a set of dissipative mechanisms that must be present in a model in order to represent properly demixing. For example, after an acceleration reversal, the TMZ should begin to demix. This physical process involves fluids falling back into regions of pure fluid, and collisions of similar fluids. A correct representation of this must be modeled as a type of dissipative process. An extension of the model in this fashion will nicely complement the models capability to describe transients in the driver that result in partial demixing. Experimental data exists for acceleration/deceleration and acceleration/coast curves with the Rocket Rig apparatus and will serve as a benchmark for this modeling enhancement.

The typical data that currently exists from mixing experiments of the Rocket Rig apparatus of AWE and Chelyabinsk-70 do not facilitate the spectral verification of our model. A similar Rocket Rig apparatus is under construction at Lawrence Livermore 
National Laboratory and should be ready for operation sometime late in 1995 (Bruce Remington and Guy DiMonte, personal communication 1995). The unique feature of this apparatus is the novel way in which data will be collected. The use of two laser sheets will enable investigators to extract evolution two-point data sets. This type of data collection will greatly aid in the verification of the spectral behavior of this model. Attention should be paid to the status of this experiment and use made of the data in order to validate extensions to this model.

Another elusive topic that deserves attention is the construction of an extension to this model that accounts for the effects of rigid walls. All spectral transport equations assumes an infinite domain over which Fourier transforms are performed and use as a constraint to modeling the fact that the correlations must go to zero as the correlation distance asymptotes to infinity. Modifications to the theory to account for finite domains should be investigated. 


\section{Appendix A. Conservation of Energy for Single-Point Transport Equations}

This Appendix addresses the issue of conservation of energy for the BHR single-point turbulence transport model which is discussed in Chapter 3. The analysis carried out in this Appendix is for the case of a mixing layer between two fluids resulting from a gravitational instability, i.e., Rayleigh-Taylor mixing. Here it is shown that the single-point turbulence transport model, which treats the flow field as a single fluid, does conserve energy. Since the mass and momentum equations are written in conservative form, it follows that momentum and mass are conserved also. The analysis of the conservation of energy is presented. It is discussed here in the context of the single-point BHR model of Chapter 3; a similar argument also exists for the spectrally integrated spectral model of Chapter 5.

Refer to Fig. A.1 for a schematic of the problem to be analyzed here. The heavy fluid is on top and the lighter fluid is on the bottom. Gravity in the downward direction sets up an adverse pressure gradient across the fluid interface, and the two fluids of the unstable configuration mix. For this analysis, it is assumed that the mixed layer (TMZ) extends to infinity in the horizontal $x$ and $z$ directions, and variations in the mean flow occur only in the vertical y direction. The differential volume of an element of the mixing layer is

$$
\delta \mathrm{V}=\mathrm{A} \delta \mathrm{y},
$$

where $\mathrm{A}$ is the area of the base of an arbitrary vertical column, and $\delta \mathrm{y}$ is the thin horizontal strip contained within the vertical column. This area, A, is treated as a constant throughout the analysis. The mean flow and the turbulent kinetic energy equations are used to demonstrate that the time rate of change of the total energy per unit volume, $\bar{\rho} E$, integrated over the volume is zero,

$$
\frac{\mathrm{d}}{\mathrm{dt}} \int_{\text {VOL }} \bar{\rho} \mathrm{E} \mathrm{dV}=\frac{\mathrm{d}}{\mathrm{dt}} \bar{\rho}(E)_{\text {TOT }}=0
$$


i.e., the model conserves energy. Here

$$
\bar{\rho} E=\bar{\rho}(\mathrm{KE})_{\mathrm{MF}}+\bar{\rho}(\mathrm{KE})_{\mathrm{TURB}}+\bar{\rho} \mathrm{PE}+\bar{\rho} \mathrm{H},
$$

in which $\mathrm{PE}$ is the potential energy per unit mass, $H$ is the heat energy per unit mass, $(\mathrm{KE})_{\mathrm{MF}}$ is the kinetic energy per unit mass of the mean flow, and (KE)TURB is the kinetic energy per unit mass of the turbulent flow.

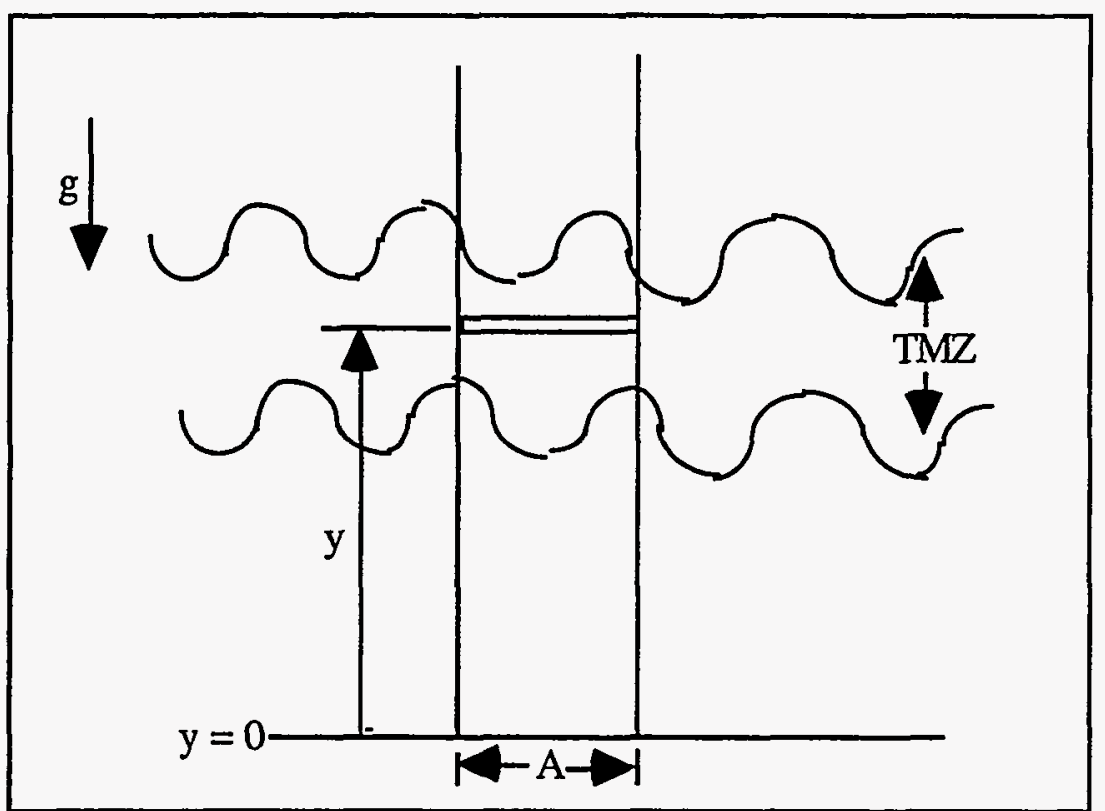

Figure A.1. Schematic of an idealized TMZ.

The separation of contributions from the mean and turbulent parts of the flow is shown in Eq. (2.2.16), and for this purpose, can be rewritten as

$$
\mathrm{KE}=(\mathrm{KE})_{\mathrm{MF}}+(\mathrm{KE})_{\mathrm{TURB}} \text {. }
$$

We assume for this demonstration that $\overline{\mathbf{u}}=0$.

Thus, we use the mean flow transport equation and the transport equation for the turbulent kinetic energy to derive a transport equation for the total kinetic energy per unit volume of the flow, $\bar{\rho} \mathrm{KE}$.

In order to derive a transport equation for $\bar{\rho}(\mathrm{KE})_{\mathrm{MF}}$, the kinetic energy per unit volume of the mean flow, we start with the averaged equation for mean flow, Eq. (2.4.8). Use is made of the conservation of mass, Eq. (2.4.9), to rearrange the form of the terms on 
the left side and the result is multiplied by $\tilde{\mathbf{u}}$ and contracted (summing repeated indices and neglecting molecular viscosity),

or,

$$
\tilde{u}_{i} \bar{p} \frac{\partial \tilde{u}_{i}}{\partial t}+\tilde{u}_{i} \bar{\rho} \tilde{u}_{n} \frac{\partial \tilde{u}_{i}}{\partial x_{n}}=-\tilde{u}_{i} \frac{\partial \bar{p}}{\partial x_{i}}-\tilde{u}_{i} \frac{\partial \overline{\rho u_{i}^{\prime \prime} u_{n}^{\prime \prime}}}{\partial x_{n}}+\bar{\rho} g_{i} \tilde{u}_{i},
$$

$$
\bar{\rho} \frac{\partial}{\partial t}\left(\frac{1}{2} \tilde{u}_{i} \tilde{u}_{i}\right)+\bar{\rho} \tilde{u}_{n} \frac{\partial}{\partial x_{n}}\left(\frac{1}{2} \tilde{u}_{i} \tilde{u}_{i}\right)=-\tilde{u}_{i} \frac{\partial \bar{p}}{\partial x_{i}}-\tilde{u}_{i} \frac{\partial \overline{\rho u_{i}^{\prime} u_{n}^{\prime \prime}}}{\partial x_{n}}+\bar{\rho} g_{i} \tilde{u}_{i} .
$$

The conservation of mass is used once again to obtain

$$
\frac{\partial}{\partial t}\left(\frac{1}{2} \bar{\rho} \tilde{u}_{i} \tilde{u}_{i}\right)+\frac{\partial}{\partial x_{n}}\left(\frac{1}{2} \bar{\rho} \tilde{u}_{i} \tilde{u}_{i} \tilde{u}_{n}\right)=-\tilde{u}_{i} \frac{\partial \bar{p}}{\partial x_{i}}-\tilde{u}_{i} \frac{\partial \overline{\rho u_{i}^{\prime \prime} u_{n}^{\prime \prime}}}{\partial x_{n}}+\bar{\rho} g_{i} \tilde{u}_{i},
$$

or, using the definitions for the kinetic energy of the mean flow and for the Reynolds stress tensor, we write:

$$
\frac{\partial \bar{\rho}(K E)_{M F}}{\partial t}+\frac{\partial \bar{\rho}(K E)_{M F} \tilde{u}_{n}}{\partial x_{n}}=-\tilde{u}_{n} \frac{\partial \bar{p}}{\partial x_{n}}-\tilde{u}_{i} \frac{\partial R_{i n}}{\partial x_{n}}+\bar{\rho} g_{n} \tilde{u}_{n} .
$$

A similar transport equation for the turbulent kinetic energy per unit volume, $\bar{\rho}(\mathrm{KE})_{\text {TURB }}$, can be derived from the transport equation for the Reynolds stress. The derivation of this transport equation is accomplished by applying Eq. (2.4.16), the definition of turbulent kinetic energy per unit mass,

$$
\mathrm{KE}_{\mathrm{TURB}}=\frac{\mathrm{R}_{\mathrm{nn}}}{2 \bar{\rho}}=\frac{\overline{\rho u_{n}^{\prime \prime} \mathrm{u}_{\mathrm{n}}^{\prime \prime}}}{2 \bar{\rho}}
$$

to the transport equation for the Reynolds stress tensor, Eq. (2.4.10) to find

$$
\frac{\partial\left(\bar{\rho} K_{\text {TURB }}\right)}{\partial \mathrm{t}}+\frac{\partial\left(\bar{\rho} K E_{\text {TURB }}\right) \tilde{\mathrm{u}}_{\mathrm{n}}}{\partial \mathrm{x}_{n}}=\mathrm{a}_{\mathrm{n}} \frac{\partial \overline{\mathrm{p}}}{\partial \mathrm{x}_{\mathrm{n}}}-\mathrm{R}_{\mathrm{mn}} \frac{\partial \tilde{\mathrm{u}}_{\mathrm{n}}}{\partial \mathrm{x}_{\mathrm{m}}}-\frac{\partial \mathrm{R}_{\mathrm{nmm}}}{\partial \mathrm{x}_{\mathrm{n}}}-\bar{\rho} \varepsilon,
$$

where $\varepsilon$ represents the irreversible conversion of turbulent kinetic energy into heat due to the pressure-velocity gradient correlations, i.e., $\bar{\rho} \varepsilon$ is a source term to the transport equation for the heat energy. By using Eq. (A.3), a transport equation for the kinetic energy per unit volume of the flow can be derived by adding Eqs. (A.8) and (A.9). 


$$
\frac{\partial \bar{\rho} K E}{\partial t}+\frac{\partial \bar{\rho} K E \tilde{u}_{n}}{\partial x_{n}}=\left(a_{n}-\tilde{u}_{n}\right) \frac{\partial \bar{p}}{\partial x_{n}}-\left(\tilde{u}_{n} \frac{\partial R_{m n}}{\partial x_{m}}+R_{m n} \frac{\partial \tilde{u}_{n}}{\partial x_{m}}\right)-\frac{\partial R_{n m m}}{\partial x_{n}}-\bar{\rho} \varepsilon+\bar{\rho} g_{n} \tilde{u}_{n} .
$$

For the case of $\overline{\mathbf{u}}=0$, and by allowing only variations in the $y$-direction, this can be written as

$$
\frac{\partial \bar{\rho} K E}{\partial t}+\frac{\partial \bar{\rho} K E \tilde{u}}{\partial y}=-\frac{\partial \tilde{u} R_{m n}}{\partial y}+\bar{\rho} g \tilde{u}-\frac{\partial R_{n m m}}{\partial y}-\bar{\rho} \varepsilon .
$$

Using Eq. (A.1), we integrate this equation over the volume of the mixing layer to obtain

$$
\mathrm{A} \int_{0}^{\infty}\left(\frac{\partial \bar{\rho} K E}{\partial t}+\frac{\partial \bar{\rho} K E \tilde{u}}{\partial y}\right) d y=A \int_{0}^{\infty}\left(-\frac{\partial \tilde{u} R_{m n}}{\partial y}+\bar{\rho} g \tilde{u}-\frac{\partial R_{n m m}}{\partial y}-\bar{\rho} \varepsilon\right) d y .
$$

The position $y=0$ is located well outside of the mixing layer (see Fig. A.1) where $R_{i j}$ and the mean flow are all zero; obviously the (KE) TURB will also be zero at this position. The position $y=0$ is also the reference from which the potential energy is measured. The same boundary conditions that apply at the position $y=0$ also apply as $y \rightarrow \infty$. With these boundary conditions, the equation reduces to

$$
\mathrm{A} \int_{0}^{\infty}\left(\frac{\partial \bar{\rho} \mathrm{KE}}{\partial \mathrm{t}}\right) \mathrm{dy}=\mathrm{A} \int_{0}^{\infty}(\bar{\rho} \mathrm{g} \tilde{\mathrm{u}}-\bar{\rho} \varepsilon) \mathrm{dy}
$$

Applying the Leibnitz rule to the partial time derivative on the left side, we arrive at an expression for the time rate of change of the total kinetic energy due to the motion of the fluid in the vertical column shown in Fig. A.1:

$$
\frac{\mathrm{d}}{\mathrm{dt}}(\bar{\rho} \mathrm{KE})_{\mathrm{TOT}}=\mathrm{A} \int_{0}^{\infty}(\bar{\rho} g \tilde{u}) \mathrm{dy}-\mathrm{A} \int_{0}^{\infty}(\bar{\rho} \varepsilon) \mathrm{dy} .
$$

Next, an expression for the time rate of change of the potential energy per unit volume, $\bar{\rho} \mathrm{PE}$, for the mixing layer is derived. From Fig. A.1 it can be seen that the potential energy per unit volume for the thin horizontal strip of fluid in the gravitational field (body force field) is

$$
\bar{\rho} \mathrm{PE}=-\left(\alpha_{1} \rho_{1}+\alpha_{2} \rho_{2}\right) \mathrm{gy},
$$


where $y$ is the height of the strip above the reference point, $y=0$, in the gravitational field, and $\mathrm{g}<0$ for a downward pointing gravity vector. The gravitational field is taken as spatially constant. (Mixing problems with a spatially varying gravitational field are beyond the scope of this study but do occur, for example in astrophysical circumstances). To derive an expression for the total potential energy of the column of fluid through the mixing layer, integrate over the volume of Eq. (A.1) and utilize the definition of $\bar{\rho}$ given in Eq. (2.2.20):

$$
(\bar{\rho} \mathrm{PE})_{\mathrm{TOT}}=\mathrm{A} \int_{0}^{\infty}(\bar{\rho} \mathrm{PE}) \mathrm{dy}=-\mathrm{A} \int_{0}^{\infty}\left(\alpha_{1} \rho_{1}+\alpha_{2} \rho_{2}\right) \mathrm{gy} \mathrm{dy} .
$$

The time rate of change of the total potential energy per unit volume is given by

$$
\frac{\mathrm{d}}{\mathrm{dt}}(\bar{\rho} \mathrm{PE})_{\text {TOT }}=-\mathrm{A} \int_{0}^{\infty} \frac{\partial}{\partial \mathrm{t}}\left[\left(\alpha_{1} \rho_{1}+\alpha_{2} \rho_{2}\right) \mathrm{gy}\right] \mathrm{dy} .
$$

With Eqs. (2.5.6) and (2.5.7), this can be rewritten as

$$
\frac{\mathrm{d}}{\mathrm{dt}}(\bar{\rho} \mathrm{PE})_{\text {TOT }}=\mathrm{A} \int_{0}^{\infty}\left[\left(\rho_{1} \frac{\partial \alpha_{1} \mathrm{u}_{1}}{\partial \mathrm{y}}+\rho_{2} \frac{\partial \alpha_{2} \mathrm{u}_{2}}{\partial \mathrm{y}}\right) \mathrm{gy}+\left(\alpha_{1} \rho_{1}+\alpha_{2} \rho_{2}\right) \mathrm{y} \frac{\mathrm{dg}}{\mathrm{dt}}\right] \mathrm{dy} \text {. }
$$

The time rate of change of the body force, $\mathrm{dg} / \mathrm{dt}$, will be neglected for these conservation of energy considerations since a changing gravitational field implies a changing point of reference, and this demonstration can be completed in its entirety without involving the additional complexities associated with this term. Thus we discard the jerk and make use of the chain rule to find

$$
\frac{\mathrm{d}}{\mathrm{dt}}(\bar{\rho} \mathrm{PE})_{\mathrm{TOT}}=\mathrm{A} \int_{0}^{\infty}\left[\left(\frac{\partial \rho_{1} g y \alpha_{1} u_{1}}{\partial y}-\alpha_{1} \mathrm{u}_{1} \frac{\partial \rho_{1} g y}{\partial y}+\frac{\partial \rho_{2} g y \alpha_{2} \mathrm{u}_{2}}{\partial y}-\alpha_{2} \mathrm{u}_{2} \frac{\partial \rho_{2} g \mathrm{y}}{\partial \mathrm{y}}\right) \mathrm{gy}\right] \mathrm{dy} \text {. }
$$

Since gravity and the densities are spatially constant and the velocities go to zero at $y=0$ and as $\mathrm{y} \rightarrow \infty$, Eq. (A.19) becomes

$$
\frac{\mathrm{d}}{\mathrm{dt}}(\bar{\rho} \mathrm{PE})_{\text {TOT }}=-\operatorname{Ag} \int_{0}^{\infty}\left(\alpha_{1} \rho_{1} \mathrm{u}_{1}+\alpha_{2} \rho_{2} \mathrm{u}_{2}\right) \mathrm{dy} \text {. }
$$

By using Eq. (2.2.29) to reexpress the momentum of the strip, we arrive at 


$$
\frac{d}{d t}(\bar{\rho} P E)_{\text {TOT }}=-A g \int_{0}^{\infty} \bar{\rho} \tilde{u} d y .
$$

The term in the transport equation for the turbulent kinetic energy which represents the dissipation to heat of the turbulent kinetic energy, $\bar{\rho} \varepsilon$, is a source in the transport equation for the heat energy per unit volume, $\bar{\rho} \mathrm{H}$. Without going into detail it will suffice to say that the remaining advective, diffusive, and other source (work) terms in the heat equation are conservative. Therefore, upon integration, the time rate of change of the total heat energy in the flow can be expressed as

$$
\frac{\mathrm{d}(\bar{\rho} \mathrm{H})_{\text {TOT }}}{\mathrm{dt}}=\mathrm{A} \int_{0}^{\infty}(\bar{\rho} \varepsilon) \mathrm{dy} .
$$

We can now use Eqs. (A.21) and (A.22) to rewrite Eq. (A.14) as

or,

$$
\frac{\mathrm{d}}{\mathrm{dt}}(\bar{\rho} \mathrm{KE})_{\text {TOT }}=-\frac{\mathrm{d}}{\mathrm{dt}}(\bar{\rho} \mathrm{PE})_{\text {TOT }}-\frac{\mathrm{d}}{\mathrm{dt}}(\bar{\rho} \mathrm{H})_{\text {TOT }} \text {, }
$$

$$
\frac{\mathrm{d}}{\mathrm{dt}}\left[(\overline{\mathrm{\rho}} \mathrm{KE})_{\mathrm{TOT}}+(\bar{\rho} \mathrm{PE})_{\mathrm{TOT}}+(\bar{\rho} \mathrm{H})_{\mathrm{TOT}}\right]=0
$$

Using Eq. (A.3), we can now show that the time rate of change of the total energy of the system is

$$
\frac{\mathrm{d}}{\mathrm{dt}}(\bar{\rho} \mathrm{E})_{\mathrm{TOT}}=0
$$

Thus, we have shown that the BHR single-point transport model for variable density flows as discussed in Chapter 3 conserves energy.

It should be noted here that only the mean flow equation and the unmodeled transport equation for the Reynolds stress tensor are necessary to show rigorously proper energy conservation. 


\section{Appendix B. Closure for the Single-Point b-Equation}

The single-point transport equation for $b$, which was derived in Chapter 3 , contains one correlation, $\overline{v^{\prime} u_{i}^{\prime}}$, that must be modeled. The authors of BHR (Besnard, et al. 1992) have chosen to model this term as a diffusion term. In this study, we have improved the modeling of this term. Through careful geometrical considerations, an expression for the time evolution of $\mathrm{b}$ can be derived. In this Appendix we demonstrate, in two different ways, that this term should not be modeled as a diffusion term but rather as a modification to the source term in the b equation.

The first way we demonstrate how the specific volume-velocity correlation should be modeled is to approach it by showing the overall kinematics of the flow through a control volume and then see what the equation lacks to be consistent with the kinematics. It is believed that what the equation lacks is then properly accounted for by the modeling of the $\overline{v^{\prime} u_{i}^{\prime}}$ correlation. The second approach will consider the configurational approach used in Chapter 2 to derive expressions for unclosed correlations. The two approaches are essentially equivalent. Both approaches suggest a consistent expression for the kinematical response of $\mathrm{b}$ to the fluxing of the two fluids by the velocity a, namely:

$$
\frac{\partial b}{\partial t}=\left(\frac{2 \bar{\rho}-\rho_{1}-\rho_{2}}{\rho_{1} \rho_{2}}\right) \frac{\partial \bar{\rho} a_{n}}{\partial x_{n}}
$$

plus the $\overline{\mathbf{u}}$ term if $\overline{\mathbf{u}} \neq 0$. (Equation (B.1) is appropriate for the case of no interspecies diffusion such that the $\hat{b}$ transport equation has no decay terms. )

For the first approach, consider a given control volume, $\delta \mathrm{V}$, located in a flow. A rigorous derivation of the changes in the quantity $b$ within the control volume can be formulated. Knowledge of the flux of each fluid through the boundary of the prescribed control volume is utilized to determine the changes in concentrations of each fluid. For an incompressible flow, an evolution equation for each species is derived from the averaged conserva- 
tion of mass, Eq. (2.2.10). The following kinematical relations are used; they relate species concentrations to their respective microscopic densities:

and the average density equation,

$$
c_{1}=\frac{\alpha_{1} \rho_{1}}{\bar{\rho}}, \quad c_{2}=\frac{\alpha_{2} \rho_{2}}{\bar{\rho}},
$$

$$
\bar{\rho}=\alpha_{1} \rho_{1}+\alpha_{2} \rho_{2} \text {. }
$$

Upon combining the above relations with the conservation of mass equation, the flux of each fluid can be determined as follows. The species concentration equations are

and,

$$
\frac{\partial \bar{\rho} c_{1}}{\partial t}+\frac{\partial \bar{\rho} c_{1} u_{1 n}}{\partial x_{n}}=0
$$

$$
\frac{\partial \bar{\rho} c_{2}}{\partial t}+\frac{\partial \bar{\rho} c_{2} u_{2 n}}{\partial x_{n}}=0
$$

The mass fluxes in these equations can be specified relative to either the Eulerian or the Lagrangian frame of reference. Using the above relations, we can express the Eulerian fluxes as

and,

$$
\bar{\rho} c_{1} u_{1 n}=\rho_{1} \alpha_{1} u_{1 n}
$$

$$
\bar{\rho} c_{2} u_{2 n}=\rho_{2} \alpha_{2} u_{2 n} \text {. }
$$

The respective fluxes for fluid one, $\overline{\mathrm{F}}_{1}$, and fluid two, $\overline{\mathrm{F}}_{2}$, are

and,

$$
\overline{\mathbf{F}}_{1}=\left(\frac{\rho_{1} \bar{\rho}}{\rho_{1}-\rho_{2}}\right) \mathbf{a},
$$

$$
\overline{\mathbf{F}}_{2}=-\left(\frac{\rho_{2} \bar{\rho}}{\rho_{1}-\rho_{2}}\right) \mathbf{a}
$$

It should be noted here that these fluxes are with respect to the laboratory frame of reference, moving with $\overline{\mathbf{u}}$, and $\overline{\mathbf{u}}=0$. For the purpose of this derivation, the mass fluxes relative to the laboratory frame of reference are used in order to identify readily fluid fluxes across a control volume surface.

Upon substitution of expressions for the masses of fluid one and fluid two, Eq. (2.2.27) and Eq. (2.2.28), into the configurational expression for b, Eq. (2.2.33), we obtain the expression, 


$$
\mathrm{b}=\frac{\mathrm{m}_{1} \mathrm{~m}_{2}\left(\rho_{1}-\rho_{2}\right)^{2}}{\rho_{1}^{2} \rho_{2}^{2} \delta V^{2}}
$$

The fluid flux across the surface of an arbitrary control volume is used to find the change in the amount of fluid in that particular control volume, i.e.,

and,

$$
\frac{\delta \mathrm{m}_{1}}{\delta \mathrm{t}}=-\oint_{\mathrm{S}} \hat{\mathbf{n}} \cdot \overline{\mathbf{F}}_{1} \mathrm{dA},
$$

or,

$$
\frac{\delta \mathrm{m}_{2}}{\delta \mathrm{t}}=-\oint_{\mathrm{S}} \hat{\mathbf{n}} \cdot \overline{\mathbf{F}}_{2} \mathrm{dA},
$$

$$
\mathrm{m}_{1}(\mathrm{t}+\delta \mathrm{t})=\mathrm{m}_{1}(\mathrm{t})-\delta \mathrm{t} \oint_{\mathrm{s}} \hat{\mathbf{n}} \cdot \overline{\mathbf{F}}_{1} \mathrm{dA}+\mathrm{O}\left(\delta \mathrm{t}^{2}\right)
$$

and,

$$
\mathrm{m}_{2}(\mathrm{t}+\delta \mathrm{t})=\mathrm{m}_{2}(\mathrm{t})-\delta \mathrm{t} \oint_{\mathrm{S}} \hat{\mathbf{n}} \cdot \overline{\mathbf{F}}_{2} \mathrm{dA}+\mathrm{O}\left(\delta \mathrm{t}^{2}\right)
$$

where $\hat{\mathbf{n}}$ is an outward unit normal vector, and $S$ is the surface area of the arbitrary control volume, $\delta$ V. Upon substitution of the definitions of the fluid fluxes, Eq. (B.4) and Eq. (B.5), and application of the divergence theorem, Equations (B.9) and (B.10) become

$$
m_{1}(t+\delta t)=m_{1}(\delta t)-\delta t\left(\frac{\rho_{1}}{\rho_{1}-\rho_{2}}\right) \int_{\delta V} \nabla \cdot(\bar{\rho} \mathbf{a}) d V
$$

and,

$$
m_{2}(t+\delta t)=m_{2}(\delta t)+\delta t\left(\frac{\rho_{2}}{\rho_{1}-\rho_{2}}\right) \int_{\delta V} \nabla \cdot(\bar{\rho} \mathbf{a}) d V
$$

Thus, Equation (B.6) reduces to

$$
b(t+\delta t)=\left[m_{1}(t)-\delta t\left(\frac{\rho_{1}}{\rho_{1}-\rho_{2}}\right) \int_{V} \nabla \cdot(\bar{\rho} \mathbf{a}) d V\right]\left[m_{2}(t)+\delta t\left(\frac{\rho_{2}}{\rho_{1}-\rho_{2}}\right) \int_{V} \nabla \cdot(\bar{\rho} \mathbf{a}) d V\right] \frac{\left(\rho_{1}-\rho_{2}\right)^{2}}{\rho_{1}^{2} \rho_{2}^{2} \delta V^{2}}
$$

To first order in $\delta \mathrm{t}$ this equation can be written as

$$
\frac{\delta b}{\delta \mathrm{t}}=\frac{\left(\rho_{1}-\rho_{2}\right)\left(\alpha_{1}-\alpha_{2}\right)}{\rho_{1} \rho_{2}}\left(\frac{1}{\delta \mathrm{V}}\right) \int_{\delta \mathrm{V}} \nabla \cdot(\bar{\rho} \mathbf{a}) \mathrm{dV} .
$$

Upon using the definition of $\bar{\rho}$, performing the volume integral, and taking the limit as $\delta \mathrm{t} \rightarrow 0$, and $\delta \mathrm{V} \rightarrow 0$, the above expression becomes 


$$
\frac{\partial \mathrm{b}}{\partial \mathrm{t}}=\left(\frac{2 \bar{\rho}-\rho_{1}-\rho_{2}}{\rho_{1} \rho_{2}}\right) \nabla \cdot(\bar{\rho} \mathbf{a}) .
$$

Equation (B.14) describes the change in $b$ in an arbitrary control volume due to the fluxing of two fluids through the surface of the control volume.

For the second approach, we rewrite the unclosed correlation, $\overline{v^{\prime} u_{\mathfrak{i}}^{\prime}}$, in terms of the configurational representation discussed in Chapter 2, namely,

$$
\overline{v^{\prime} u_{i}^{\prime}}=\alpha_{1}\left(\frac{1}{\rho_{1}}-\bar{v}\right)\left(u_{1 i}-\bar{u}_{i}\right)+\alpha_{2}\left(\frac{1}{\rho_{2}}-\bar{v}\right)\left(u_{2 i}-\bar{u}_{i}\right) .
$$

After some algebra it is found that

$$
\overline{v^{\prime} u_{i}^{\prime}}=\frac{\alpha_{1} \alpha_{2}\left(u_{1 i}-u_{2 i}\right)\left(\rho_{2}-\rho_{1}\right)}{\rho_{1} \rho_{2}} .
$$

Applying the configurational definition of a from Chapter 2 to Eq. (B.16), we can express the entire unmodeled term in the $b$ equation as

$$
\bar{\rho} \frac{\partial \overline{v^{\prime} u_{n}^{\prime}}}{\partial x_{n}} \rightarrow-\frac{\bar{\rho}}{\rho_{1} \rho_{2}} \frac{\partial \bar{\rho} a_{n}}{\partial x_{n}} .
$$

Combining this term with the pre-existing unmodeled term in the b equation, namely,

and making use of the relations,

$$
\frac{b+1}{\bar{\rho}} \frac{\partial \bar{p} a_{n}}{\partial x_{n}}
$$

and

$$
\alpha_{1}+\alpha_{2}=1 \text {, }
$$

$$
\bar{\rho}=\alpha_{1} \rho_{1}+\alpha_{2} \rho_{2},
$$

lead to the following expression for the temporal evolution of $b$ (for the case of $\overline{\mathbf{u}}=0$.);

$$
\frac{\partial b}{\partial t}=\left(\frac{2 \bar{\rho}-\rho_{1}-\rho_{2}}{\rho_{1} \rho_{2}}\right) \frac{\partial \bar{\rho} a_{n}}{\partial x_{n}} .
$$

Thus it is shown that both approaches give the same form for modeling of $\overline{v^{\prime} u_{i}^{\prime}}$.

This derivation provides an improvement in the current single-point transport equation for $\mathrm{b}$ in the BHR model (Besnard, et al. 1992). This improvement is carried over into Chapter 5 where it is incorporated into our new spectral theory. In Chapter 5 it is assumed that the spectral mass-fluxing velocity, $a$, creates spectral $b$ at the same scales. 


\section{Appendix C. Categorizing Two-Fluid Interpenetration}

Chapter 2 defined most of the important flow variables that are relevant to this study from a configurational approach. In this section a thought experiment is proposed to illustrate some of the kinematical processes by which mass is fluxed in a mixing process. As was the case in Chapter 2, this appendix is also designed to be used as a tool for visualizing some of the underlying ideas used to create a model to describe the turbulent mixing of two fluids.

There are two types of processes by which two immicible fluids can mix under the influence of a gravitational field oriented in such a way as to make the two-fluid configuration unstable. In this study we define these two types of processes as an ordered process and a disordered process. Both are component parts of the mixing process in the Rayleigh-Taylor instability.

The first mixing process to be discussed, namely the ordered process, is exemplified in pictures of the early stages of the Rayleigh-Taylor instability. These pictures show that as the lighter fluid is accelerated into the heavier fluid, spikes of the heavier fluid penetrate into the lighter fluid and the lighter fluid bubbles up into the heavier fluid. This is what we define as the ordered mixing process, and it is anisotropic.

An extreme example of this ordered process of interpenetration is the case of widely dispersed marbles falling through a homogeneous fluid. If there is no interaction between the falling marbles, that is to say, no marble-marble interactions which would alter the motion of the marbles, then the marbles fall in an orderly fashion. This example is given here so as to give an intuitive feeling for the type of motion that is being categorized as orderly. The twofield flow equations discussed in Chapter 4 are very useful in describing this dispersed-marble type of purely ordered interpenetration. With two fluids the purely-ordered form of interpenetration idealization is approached, as shown by Daly (1969), in the case of two very viscous fluids with large surface tension, for which the interpenetration takes place in long, slender fingers. 
Now let us introduce the concept of a disordered flow by increasing the volume fraction of marbles in the above example enough so as to initiate marble-marble influences on one another as the marbles fall. This causes an additional component of the marbles trajectory that deviates from the direction of the pressure gradient, namely, lateral fluctuations in the flow that are characterized in this study as the onset of a disordered component of the flow. In most real circumstances the entire flow is comprised of the ordered plus the disordered components.

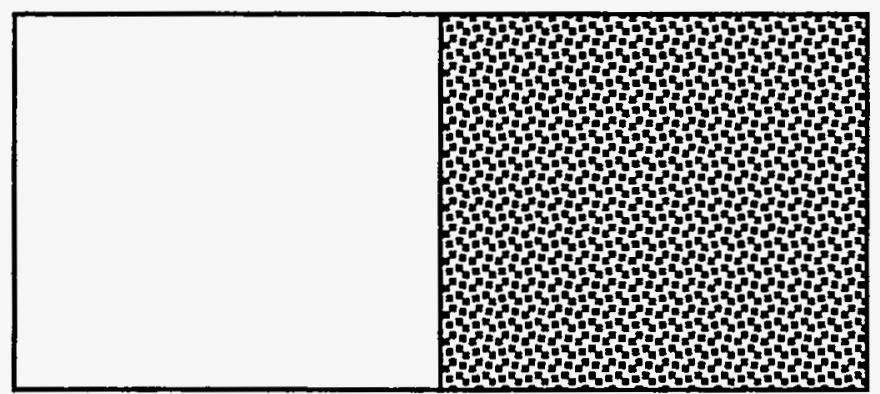

Figure C.1. Schematic of a two-dimensional box filled with sandy liquid on the right side and pure liquid on the left side separated by a vertical partition.

To illustrate an example of disordered mixing that is of relevance to this study, consider a box filled with a pure liquid in one half partitioned from a sandy liquid in the other half. (See Fig. C.1 ) Each liquid is then stirred well until both are completely turbulent. First, let us examine the case in which there is no body force induced by gravity on the liquids in the box, i.e., $g=0$. In this case when the partition is instantaneously removed, the two liquids mix by diffusive processes only. That is to say, the mixing of the two species can occur without any coupling to, or help from, gravitational body forces. The condition of incompressibility dictates that the diffusive process will flux equal volumes of each material across the centerline which creates a net mass flux at this centerline. This type of random walk interpenetration of the two fluids is considered to be highly disordered and is directed along the average density gradient. This disordered process appears in the a equation, for evolution of the net mass flux, as a source due to the random-walk-like nature of the diffusive 
process. Since turbulence is a highly dissipative diffusive process, traditional modeling choices (Daly \& Harlow, 1970) take this process into account when modeling certain higher order correlations by gradient-flux approximations.

Extend this example to the case with the same setup as in Fig. C.1 but now gravity is turned on in such a way as to create a pressure gradient in the box resulting in higher pressure in the pure (lighter) fluid (see Fig. C.2). The arrow is pointed in the direction of the gravitational body force. This configuration is unstable.

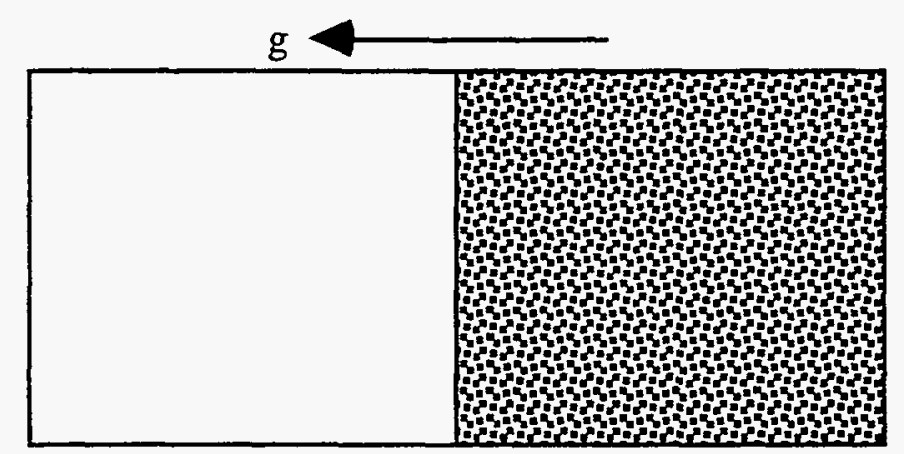

Figure C.2. Schematic of a two-dimensional box filled with pure liquid on the left side of the partition and sandy liquid on the right side of the partition. Gravity produces a pressure gradient across the interface such that when the partition is removed the highest pressure will be in the pure liquid.

If the two fluids are initially stirred, they will still mix in the same diffusive manner as in the above example, but the mixing process is now augmented by a gravity driven interpenetration. Like the above scenario, this process is also highly anisotropic. The preferential direction of this anisotropy is dictated by the external drivers, i.e., gravity and the density gradient. The ordered part of the mix is described as a pressure gradient driven source in the a equation of the turbulence transport model.

A third scenario must be mentioned here for completeness, and this is the case in which the gravity field of the second example is suddenly reversed after some mixing has already taken place. Before the gravity is reversed and the demixing of the two fluids commences, both the density and pressure gradient terms of the a equation work in tandem to mix the two fluids. Once the gravity is reversed, however, the pressure gradient term re- 
verses sign instantaneously, and the density gradient term maintains the same sign as before the gravity reversal. Thus the rate of demixing depends on a balance between these two terms in the a equation. This is discussed in Section 7.3.

The above examples show that two very different mechanisms influence the mixing of two immiscible fluids. Due to the nature of both the single-point turbulence equations and the two-phase flow equations, neither set is independently capable of describing all facets of the mixing process described above. For this reason, hybrid type models (Cranfill, 1991 \& 1992) have been proposed which work reasonably well for describing mixing as well as demixing.

Keeping the above discussion in mind, ideas that we are trying to convey in certain sections of the text might come a little easier. This section was included to highlight the issues that arise when attempting to model the different possible ways in which fluids can be fluxed. This issue was investigated as background work using the single-point turbulence transport model of Chapter 3 and the two-fluid model of Chapter 4. This issue also arises in the modeling strategy used for the two-point spectral model that is introduced in Chapter 5. 


\section{Appendix D. Self-Similar Form Functions}

The concept of statistical self-similarity is demonstrated in Chapter 7 for the example of a turbulent mixing zone between two immiscible fluids. As an idealization, the zone extends laterally in all directions and all quantities are statistically independent of the lateral dimensions. Indeed we shall assume that ensemble averaging can be replaced by lateral spatial averaging, with results that vary in time only as functions of wave number and the distance normal to the layer. Such an idealization does not exist in nature, but there are nevertheless numerous circumstances in which it serves as a good approximation.

Besnard, et al. (1990) showed that statistical self-similarity is a crucial element for the validity of single-point turbulence transport models. In order to describe with a small number of variables the collective effects of a virtually infinite number of degrees of freedom, it is clear that severe constraints must exist to confine the dynamics of the fluid to a very small set of all possibilities. Moreover, one of the necessities for single-point applicability is that the return to self-similarity occur rapidly after a change in the external drive conditions.

Self-similarity of the statistics is characterized by statistical form functions. They always occur with the same structure for each set of mean flow circumstances after proper space and time scalings are performed. Universal form functions, however, are idealizations that never precisely occur in nature, although in many situations of interest they are closely approximated. The forms of such functions may strongly reflect the existence of anisotropy and may even depend on persisting conditions in the limit as $|\mathbf{k}| \rightarrow 0$. Our ultimate goal is to identify these form functions in six-dimensional $\mathbf{k}, \mathbf{x}$ space. Subsets can be found in $\mathbf{k}$ or $\mathbf{y}$ space alone and in various other combinations of the wave number and position-vector components. Complete analytical derivation is difficult (see Besnard, et al. 1990); partial derivations are well known for simple cases (e.g., the $k^{-5 / 3}$ inertial range for $\mathrm{R}_{\alpha \alpha}$; more general extraction may only be possible numerically as in Chapter 7). 
The simplest statistical self-similar forms occur in the decay of homogeneous isotropic decaying turbulence in a constant density fluid with vanishing viscosity. This form and an anisotropic generalization thereof are used to derive spectral moments as discussed by Besnard, et al. (1990). They show that with this simple idealization, the single-point equations (e.g., K$\varepsilon$ models) can be derived. These types of single-point equations have been successfully applied to circumstances far beyond the case of homogeneous anisotropic decay.

In general, self-similarity for the behavior of some turbulence statistic like $R_{i j}(y, k, t)$, means that a scaling law can be found for its variations in magnitude, such that the scaled quantity is then a function only of the combined variables, $y / L(t)$ and $k L(t)$, in which $L(t)$ is a function with the dimensions of length. Thus the function of three variables, $y, k$, and $t$, has been transformed to a function of two variables.

This scaling of magnitudes and the independent variables that define self-similarity can be described more precisely. Consider the behavior of

$$
E(y, k, t)=\frac{1}{2 \bar{\rho}} R_{n n}(y, k, t),
$$

which has the dimensions of (distance) $)^{3 /(t i m e)^{2}}$. Suppose we scale the space and time coordinates such that

and

$$
\begin{aligned}
& y \rightarrow \omega^{n} y, \\
& k \rightarrow \omega^{-n} k,
\end{aligned}
$$

$$
t \rightarrow \omega^{m_{t}},
$$

where $\omega$ is a dimensionless number that defines the scale, while $\mathrm{m}$ and $\mathrm{n}$ are numbers to be specified. We also scale the magnitude of $E$ according to its dimensionality,

$$
E \rightarrow \omega^{3 n-2 m} E .
$$

Our constraint of self-similarity in this process results in the equation

$$
\omega^{3 \mathrm{n}-2 \mathrm{~m}} E(k, y, t)=E\left(\omega^{\mathrm{n}} \mathrm{y}, \omega^{-\mathrm{n}} k, \omega^{\mathrm{m}} \mathrm{t}\right) .
$$

Differentiate with respect to $\omega$ and then set $\omega=1$. The result is 


$$
(3 n-2 m) E=n y \frac{\partial E}{\partial y}-n k \frac{\partial E}{\partial k}+m t \frac{\partial E}{\partial t},,
$$

which can be solved to give

$$
E(y, k, t)=t^{\frac{3 n-2 m}{m}} \hat{F}\left[\frac{y}{t^{n / m}}, k t^{n / m}\right],
$$

in which $\hat{\mathrm{F}}$ is an arbitrary function of its arguments. The origin of time is arbitrary, so that we may choose the onset of self-similarity at some time, $\mathrm{t}_{0}$, write

and

$$
\mathrm{L}(\mathrm{t}) \equiv \mathrm{L}_{0}\left(\mathrm{t}-\mathrm{t}_{0}\right)^{\mathrm{n} / \mathrm{m}},
$$

$$
K(t) L(t) \equiv K_{0} L_{0}\left(t-t_{0}\right)^{\frac{3 n-2 m}{m}} \text {, }
$$

and express the solution in the form,

$$
E(y, k, t)=K(t) L(t) F\left[\frac{y}{L(t)}, k L(t)\right]
$$

where the function $\mathrm{F}$ has absorbed some constants and is an arbitrary function of its arguments. Note that from this form

where

$$
\int_{0}^{\infty} E(y, k, t) d k=K(t) G\left(\frac{y}{L(t)}\right),
$$

$$
G(\eta) \equiv \int_{0}^{\infty} F(\eta, \xi) d \xi
$$

This form for $\mathrm{E}(\mathrm{y}, \mathrm{k}, \mathrm{t})$ describes a constraint of self-similarity It is derived without reference to the transport equation for $\mathrm{E}$ and serves to distinguish from all possible solutions a particular subset with a type of self-similarity.

Dimensional arguments for the spectrally integrated quantities are based on the TMZ growth having "forgotten" all finite scale details of the initial conditions so that $g$ is the only dimensional scaling parameter. Thus any velocity scale must vary as gt while the turbulence energy per unit mass varies as $(\mathrm{gt})^{2}$ and length scales vary as $\mathrm{gt}^{2}$. Thus $\mathrm{n}$ and $\mathrm{m}$ are constrained to $\mathrm{n}=2 \mathrm{~m}$. 
Applied to homogeneous, isotropic turbulence (independent of y), the result of selfsimilarity is conversion of a partial differential equation for $E(k, t)$ to an ordinary differential equation for $F[k L(t)]$. Besnard, et al. (1990) discuss in detail the properties of the function $F$.

For the mixing-layer analysis in $y-k$ space, the self-similarity constraint has likewise reduced the number of independent variables (see Appendix E). but insertion into the transport equation nevertheless results in a partial differential equation for $F(y / L, k L)$, which has a much greater richness of possible solutions than the ordinary differential equation for homogeneous, isotropic circumstances. We thus see hints of possible nonuniqueness of self-similar mixinglayer turbulence.

We are, however, concerned with extensions to the self-similar form in our special case of the mixing layer. We rewrite Eq. (D.8) with a different combination of variables in the F function (which is perfectly allowable), i.e.,

$$
E(y, k, t)=K(t) L(t) F\left[\frac{y}{L(t)}, k L(t) G\left(\frac{y}{L(t)}\right)\right] .
$$

We hypothesize that the $\mathrm{G}$ function can be found in such a way that

$$
F\left[\frac{y}{L(t)}, k L(t) G\left(\frac{y}{L(t)}\right)\right]=F_{1}\left(\frac{y}{L(t)}\right) F_{2}\left[k L(t) G\left(\frac{y}{L(t)}\right)\right] .
$$

This hypothesis, which merely suggests that $k_{\max }$ if a function of $y$, is capable of being tested numerically. It states in effect that the spectrum is self-similar in k-space with a shift in maximum and position thereof for which $k L(t)$ depends only on $\eta$, where $\eta=y / L(t)$. The first realization of this form was described by Besnard, et al. (1990) on the basis of numerical solutions of the spectral BHRZ model equations for a constant-density fluid in a temporally evolving shear layer. Chapter 7 of this report describes a further realization for the turbulent mixing of two fluids.

We are led to define a length scale $L(y, t)$ that varies with both position and time, as was assumed by Besnard, et al. (1990) in the BHRZ report (LA-11821-MS) for the spectralmoment derivations of single-point turbulence transport equations. Thus we define $L(y, t)$ as 
being equal to $L(t) G(\eta)$, and $K(y, t) L(y, t)$ as being identically equal to $F_{1}(\eta) L(t) K(t)$. Then Eqs. (D.11) and (D.12) can be written as

$$
E(y, k, t)=K(y, t) L(y, t) F_{2}[k L(y, t)] .
$$

Even more generally, if the mixing layer is of finite lateral extent or curved away from planarity, we postulate the concept of localized self-similarity with $K(x, t)$ and $L(x, t)$, which is the actual form used for deriving moment equations for turbulence in a constant density fluid. 


\section{Appendix E. Moments of the Spectral Equations}

With the spectral transport equations of Chapter 5, i.e., Eqs. (5.3.19), (5.3.26), (5.3.28), and (5.3.29), it is possible to solve a much wider scope of problems than can be addressed with single-point (nonspectral) formulations. Rapid variations in drive, for example, distort the nearly self-similar form functions into strongly circumstance-dependent expressions. Spectral equations work fine for describing such processes, but spectrallyintegrated moment equations, based on assumed persistence of self-similarity, may exhibit large errors for such applications.

Spectral transport formulations are more complicated, however, as well as being much more expensive to use in numerical investigations with high-speed computers. Thus we use the two approaches in complementary fashion; in particular, we look at the spectral approach for clues to improving and extending the moment equations.

In Chapter 3, the single-point turbulence transport equations in terms of previous nonspectral derivations (the BHR model of Besnard, et al. 1987) are discussed, to which some important clarifications and extensions have been discussed. Significant difficulties are also described, especially in regard to size scales associated with both the turbulence and the interfacial configuration of mixing fluids. Decay of turbulence, for example, is directly related to the transfer rate from low to high wave numbers. Sources of turbulence can be strong in the "active" scales and weak in the "passive" scales, the latter simply balancing the buoyancy forces with drag. The resolution of these and other questions leads directly to the spectral formulations; the inverse process takes the spectral equations back to single-point form but augmented with representations of the principal spectral improvements that were previousiy missing.

Before proceeding with the derivations, we describe an example of the new single-point augmentation. For the simple self-similar mixing layer that dominates the considerations of this report, it is arguable that single-point transport equations are not required for both $a_{i}$ and 
$R_{i j}$. They are, after all, scaled in dimensionality by the same quantity, $g$; and the spectral functions on which they are based are of universal form, so that knowledge of one implies that the other is known. Moreover, the scales associated with $b(k), a_{i}(k)$, and all the components of $R_{\mathrm{ij}}(\mathrm{k})$ must be directly proportional to each other with universal ratios. Thus a pair of single-point equations for $\mathrm{a}_{\mathrm{i}}$ and $\mathrm{L}_{\mathrm{a}}$ should be sufficient to determine the entire process. These ideas are amply verified in the examples shown in Chapter 7.

Those examples show, however, that the moment self-similarity is destroyed (e.g., through reversal of $g$ ), and the relationship among $a_{i}$ and the components of $R_{i j}$ are at once lost. Each evolves in its own particular response to the new circumstances, returning to a unique, universal relationship to each other only when (and if) self-similarity is again established (which is not the case during the de-mixing process), in which the width of the layer at the instant of $\mathrm{g}$ reversal is firmly implanted as an additional dimensional quantity. In addition, the length scales associated with each of the spectra are now no longer universally proportional to each other. If the driver continues to vary in capricious fashion, these independent behaviors continue; and the discernment of relationships among variables requires the simultaneous transport analysis of them all.

The spectral equations describe all these "independent" but interactive behaviors. Can single-point (spectral-moment) equations do likewise? There is evidence to suggest that they can do fairly well in this regard. Simple $R_{\mathrm{ij}}-\varepsilon$ models (Rotta, 1951; Daly \& Harlow, 1970; Hanjalic \& Launder, 1972) describe at least some types of transient circumstances, despite their derivation being equivalent to moments of the spectral form functions.

At least two things must contribute to the success of single-point (nonspectral) formulations. One of these is the tendency for spectra to vary principally in magnitude during many kinds of transients, with little change in form (for example, a during g reversal in Chapter 7). The second is the tendency for rapid return to self-similarity after a transient change in drive. This latter, indeed, furnishes a significant criterion for spectral-moment 
transport validity in terms of the competition between change in rate of drive and return rate to self-similarity. With the spectral equations, this competition can be examined in detail.

In this section we first show the spectral moment derivations for the simplest TMZ, in which all ensemble averaged (layer-averaged) quantities are functions of $y, k$, and $t$ only. We write

and

$$
\begin{aligned}
a_{i}(y, k, t) & =a_{i}(y, t) L_{a}(y, t) F_{a}\left(k L_{a}(y, t)\right), \\
b(y, k, t) & =b(y, t) L_{b}(y, t) F_{b}\left(k L_{b}(y, t)\right),
\end{aligned}
$$

$$
R_{i j}(y, k, t)=2 \bar{\rho}(y, t) K(y, t) L_{R}(y, t) F_{i j}\left(k L_{R}(y, t)\right) \text {. }
$$

Here, $K(y, t)$ denotes the turbulent kinetic energy and as usual, $\bar{\rho}(y, t)$ is the average density. The wave-number, $k$, occurs only in the self-similar form functions, the Fs. The other quantities, $a_{i}, b, K, L_{a}, L_{b}$, and $L_{R}$, are all functions of $y$ and $t$; our goal in this section is to derive spectral moment equations to describe their transport.

There are an infinite number of possible spectral moments one can take of our spectral equations of Chapter 5. One of these is the weighting function, $\mathrm{k}^{0}(\equiv 1.0)$. Another is $\mathrm{k}^{\mathrm{m}}$, for which Besnard et al. (1990) suggest a value of the exponent, $\mathrm{m}$, that is close to -1.0 .

The spectral-moment transport equations that we require are six in number, two for each of the spectral equations for $a_{i}, b$, and $R_{n n}$, since each has two unknown functions of position and time, a magnitude, and a length scale. (At this stage we assume that the difference between $R_{n n}$ and $R_{y y}$ is sufficiently represented by their two different forms, $F_{n n}$ and $F_{y y}$.)

We substitute the self-similar forms of Eq. (E.1) into the spectral transport equations of Chapter 5, i.e., Eqs. (5.3.19), (5.3.26), (5.3.28), and (5.3.29), and integrate over $\mathrm{dk}$ to get evolution equations for $\mathrm{K}(\mathrm{y}, \mathrm{t}), \mathrm{a}_{\mathrm{i}}(\mathrm{y}, \mathrm{t})$, and $\mathrm{b}(\mathrm{y}, \mathrm{t})$. The resulting transport equations depend on time and the physical-space variable, $y$, only. Due to the self-similar forms of Eq. (E.1) length scales for each of the variables are incorporated into the new single-point transport equations. These length scales, namely $L_{a}, L_{b}$, and $L_{R}$, may be considered the inverse of the dominant wave number $k_{\max }$ of the spectrum for each of the variables. In addition to performing the integrations just described, we also integrate the spectral transport 
equations over $\mathrm{k}^{\mathrm{m}} \mathrm{dk}$ for $\mathrm{m}=-1$ to get transport equations relating the single-point quantities, namely, $K(y, t), a_{i}(y, t)$, and $b(y, t)$, to their associated length scales.

The functions are normalized to

$$
\int_{0}^{\infty} F_{\mathrm{a}}(\xi) \mathrm{d} \xi=\int_{0}^{\infty} \mathrm{F}_{\mathrm{b}}(\xi) \mathrm{d} \xi=\int_{0}^{\infty} \mathrm{F}_{\mathrm{nn}}(\xi) \mathrm{d} \xi=1 .
$$

The maximum value of all three form functions is unity corresponding to the position $\xi=1$.

The process of substituting the self-similar forms from Eq. (E.1) into the spectral transport equations and performing the integrations discussed above is straightforward. The local cascade terms are the only terms that warrant closer examination. The integration will be shown for the cascade of a generic variable, $\varphi$.

The generic cascade terms of our model are expressed as

$$
\frac{\partial}{\partial \mathrm{k}}\left[\mathrm{k}^{2} \sqrt{\frac{\mathrm{kR}}{\bar{\rho}}}\left(-\mathrm{C}_{1} \varphi+\mathrm{C}_{2} \mathrm{k} \frac{\partial \varphi}{\partial \mathrm{k}}\right)\right],
$$

Taking the $m$-th moment of this cascade term gives

where

$$
\int_{0}^{\infty} k^{m} \frac{\partial G(k)}{\partial k} d k
$$

$$
G(k)=k^{2} \sqrt{\frac{k R_{n n}}{\bar{\rho}}}\left(-C_{1} \varphi+C_{2} k \frac{\partial \varphi}{\partial k}\right) .
$$

Upon integrating by parts, it is found that

$$
\int_{0}^{\infty} k^{m} \frac{\partial G(k)}{\partial k} d k=\left.\left(k^{\prime}\right)^{m} G\left(k^{\prime}\right)\right|_{k^{\prime} \rightarrow \infty}-m \int_{0}^{\infty} k^{m-1} G(k) d k .
$$

For this model, a good approximation for the high wave number behavior of the three quantities $a_{j}, b$, and $R_{n n}$ is that all three behave as $k^{n}$, where $n$ is at most $-5 / 3$. $G(k)$ remains constant for large values only when one samples the spectrum far enough to the right [away from the influence of $a_{i}(k)$ ]. Upon examination of $G(k)$, it can then be seen that $G(k)$ approaches a constant value as $\mathrm{k} \rightarrow \infty$; thus, for all values of $\mathrm{m}$ such that $\mathrm{m} \leq 0$, only the $\mathrm{m}=0$ moment will result in a nonzero contribution from the first term on the right side of 
Eq. (E.6). Therefore, the moment integral of the cascade terms can be expressed as

$$
\int_{0}^{\infty} k^{m} \frac{\partial G(k)}{\partial k} d k=\left\{\begin{array}{ll}
-m \int_{0}^{\infty} k^{m-1} G(k) d k & ; \text { for } m \neq 0 \\
\left.k^{2} \sqrt{\frac{k R_{n n}}{\bar{\rho}}}\left(-C_{1} \varphi+C_{2} k \frac{\partial \varphi}{\partial k}\right)\right|_{k \rightarrow \infty} & ; \text { for } m=0
\end{array} .\right.
$$

The term resulting from the moments for $m \neq 0$ on the right side of Eq. (E.7) is a constant that represents the decay of the quantity $\varphi$ due the cascade action of the turbulence to higher and higher wave numbers.

With the discussion of the cascade terms completed, the self-similar forms from Eq. (E.1) are now substituted into our spectral model equations of Chapter 5, i.e., Eqs. (5.3.19), (5.3.26), (5.3.28), and (5.3.29), and the $m=0$ and $m=-1$ moments are taken. The resulting three transport equations for $a(y, t), b(y, t)$, and $K(y, t)$ are

$$
\begin{gathered}
\frac{\partial \bar{\rho} a}{\partial t}+\frac{\partial \bar{\rho} a \tilde{u}}{\partial y}=b \frac{\partial \bar{p}}{\partial y}-\left[N_{4} \sqrt{a a}+I_{2} \sqrt{2 K\left(\frac{L_{R}}{L_{a}}\right)}\right] \frac{\bar{\rho} a}{L_{a}} \\
-N_{3} K \frac{\partial \bar{\rho}}{\partial y}+C_{d} \frac{\partial}{\partial y} \bar{\rho} v_{t} \frac{\partial a}{\partial y} \\
\frac{\partial b}{\partial t}=\left(\frac{2 \bar{\rho}-\rho_{1}-\rho_{2}}{\rho_{t} \rho_{2}}\right) \frac{\partial \bar{\rho} a}{\partial y}+I_{1} \frac{b}{L_{b}} \sqrt{2 K\left(\frac{L_{R}}{L_{b}}\right)}+C_{d} \frac{\partial}{\partial y} v_{t} \frac{\partial b}{\partial y},
\end{gathered}
$$

and

$$
\begin{aligned}
\frac{\partial \bar{\rho} K}{\partial t}+\frac{\partial \bar{\rho} K \tilde{u}}{\partial y}= & \int_{-\infty}^{+\infty} a\left(y^{\prime}\right) \frac{\partial \bar{p}\left(y^{\prime}\right)}{\partial y} S_{0}\left(y, y^{\prime}\right) d y^{\prime}-N_{3} \bar{\rho} K \frac{\partial \tilde{u}}{\partial y} \\
& +C_{d} \frac{\partial}{\partial y} v_{t} \frac{\partial \bar{\rho} K}{\partial y}-N_{2} \bar{\rho} \varepsilon .
\end{aligned}
$$


The corresponding three transport equations for their respective length scales are

$$
\begin{gathered}
\frac{\partial \bar{\rho} L_{a}}{\partial t}+\frac{\partial \bar{\rho} L_{a} \tilde{u}}{\partial y}=\left[\left(\frac{N_{7}}{N_{8}}\right)\left(\frac{L_{b}}{L_{a}}\right)-1\right] \frac{b L_{a}}{a} \frac{\partial \bar{p}}{\partial y}-I_{4} \bar{\rho} \sqrt{2 K\left(\frac{L_{R}}{L_{a}}\right)} \\
+\left[N_{3}-N_{6}\left(\frac{L_{R}}{L_{a}}\right)\right] \frac{K_{a}}{a} \frac{\partial \bar{\rho}}{\partial y}+C_{d} \frac{\partial}{\partial y} \bar{\rho} v_{t} \frac{\partial L_{a}}{\partial y} \\
-N_{5} \bar{\rho} \sqrt{a a}+\frac{2 C_{d} \bar{\rho} v_{t}}{a}\left(\frac{\partial a}{\partial y}\right)\left(\frac{\partial L_{a}}{\partial y}\right),
\end{gathered}
$$$$
\frac{\partial L_{b}}{\partial t}=\left(\frac{2 \bar{\rho}-\rho_{1}-\rho_{2}}{b \rho_{\mathrm{I}} \rho_{2}}\right)\left[\frac{N_{8}}{N_{7}} \frac{\partial \bar{\rho} \mathrm{aL}_{\mathrm{a}}}{\partial \mathrm{y}}-\mathrm{L}_{\mathrm{b}} \frac{\partial \bar{\rho} \mathrm{a}}{\partial \mathrm{y}}\right]+\mathrm{C}_{\mathrm{d}} \frac{\partial}{\partial \mathrm{y}} v_{\mathrm{t}} \frac{\partial \mathrm{L}_{\mathrm{b}}}{\partial \mathrm{y}}+\mathrm{I}_{3} \sqrt{2 \mathrm{~K}\left(\frac{\mathrm{L}_{\mathrm{R}}}{\mathrm{L}_{\mathrm{b}}}\right)}
$$$$
-\frac{N_{8}}{N_{7}} C_{f b}\left(\frac{\bar{v}^{2} \mathrm{aL}_{\mathrm{a}}}{\mathrm{b}}\right) \frac{\partial}{\partial \mathrm{y}}\left(\frac{\bar{\rho}}{\bar{v}}\right)+\frac{2 \mathrm{C}_{\mathrm{d}} v_{\mathrm{t}}}{\mathrm{b}}\left(\frac{\partial \mathrm{L}_{\mathrm{b}}}{\partial \mathrm{y}}\right)\left(\frac{\partial \mathrm{b}}{\partial \mathrm{y}}\right)
$$

and

$$
\begin{array}{r}
\frac{\partial \bar{\rho} L_{R}}{\partial t}+\frac{\partial \bar{\rho} L_{R} \tilde{u}}{\partial y}=\frac{1}{K} \int_{-\infty}^{+\infty} a\left(y^{\prime}\right) \frac{\partial \bar{p}\left(y^{\prime}\right)}{\partial y} S\left(y, y^{\prime}\right) d y^{\prime}-N_{9} \bar{\rho} L_{R} \frac{\partial \tilde{u}}{\partial y}+N_{10} \bar{\rho} \sqrt{K} \\
+\bar{\rho} C_{d} \frac{\partial}{\partial y} v_{t} \frac{\partial L_{R}}{\partial y}+\frac{2 C_{d} v_{t}}{K}\left(\frac{\partial \bar{\rho} K}{\partial y}\right)\left(\frac{\partial L_{R}}{\partial y}\right)
\end{array}
$$

where

$$
\begin{aligned}
& N_{1}=\int_{0}^{\infty}\left(\frac{1}{\xi}\right) F_{n n}(\xi) d \xi, \\
& N_{2}=\left[\xi^{2} \sqrt{2 \xi F_{n n}(\xi)}\left(C_{1} F_{n n}(\xi)-C_{2} \xi F_{n n}^{\prime}(\xi)\right)\right]_{\xi \rightarrow \infty}, \\
& N_{3}=2 \int_{0}^{\infty} F_{y y}(\xi) d \xi, \\
& N_{4}=C_{\mathrm{rpl}} \int_{0}^{\infty} \xi^{2} F_{a}^{2}(\xi) d \xi, \\
& N_{5}=\left(\frac{C_{\mathrm{rp} 1}}{N_{8}}\right) \int_{0}^{\infty} \xi F_{a}^{2}(\xi) d \xi-N_{4},
\end{aligned}
$$


产

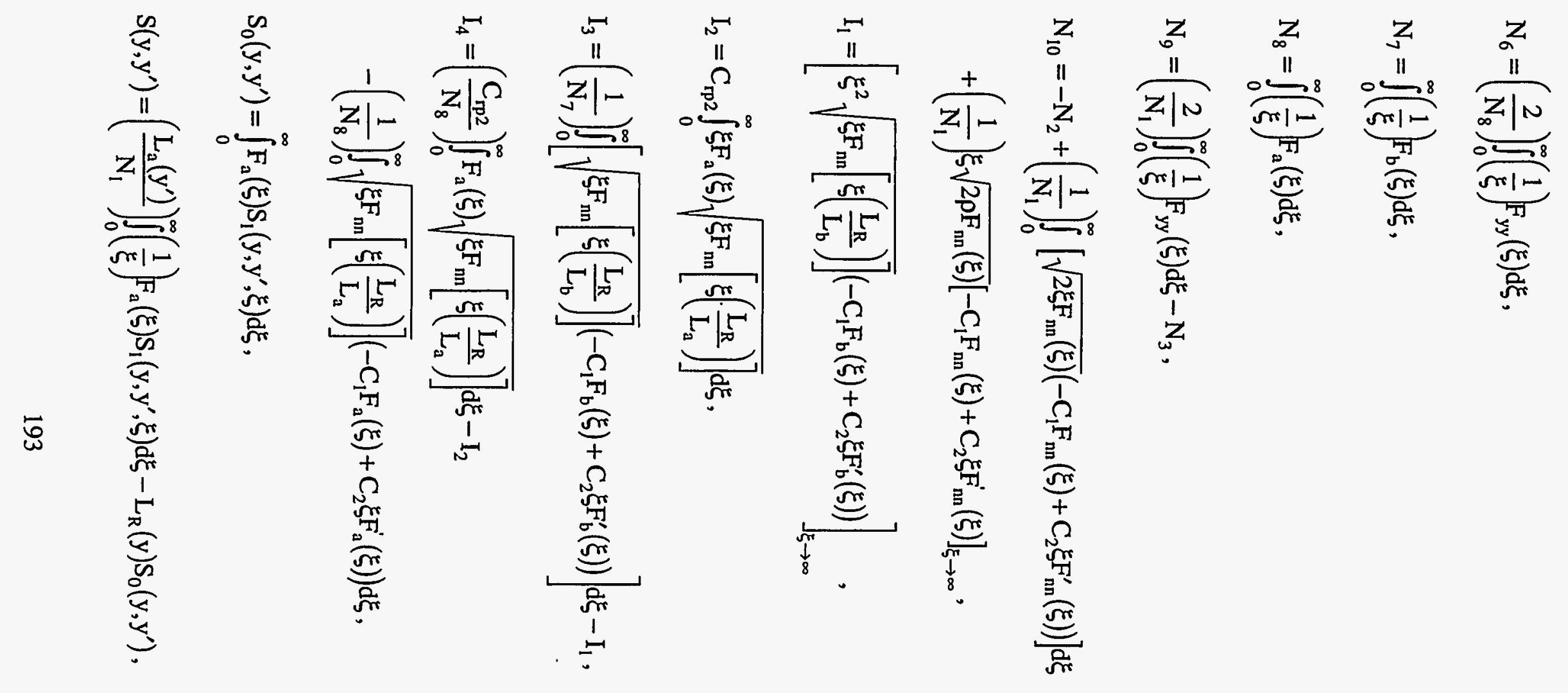


with

$$
S_{1}\left(y, y^{\prime}, \xi\right)=\frac{\exp \left[-2 \xi\left|\frac{y^{\prime}-y}{L_{a}\left(y^{\prime}\right)}\right|\right]}{\int_{-\infty}^{+\infty} \exp \left[-2 \xi\left|\frac{y^{\prime \prime}-y}{L_{a}\left(y^{\prime}\right)}\right|\right] d y^{\prime \prime}}
$$

and

$$
\varepsilon=\frac{\mathrm{K} \sqrt{\mathrm{K}}}{\mathrm{L}_{\mathrm{R}}} .
$$

Note that the decay term due to molecular diffusion in the $b(y, t)$ has been omitted since we have neglected this effect in this study. Note the persistence of the nonlocality in both the transport equation for $K(y, t)$ and its length scale $L_{R}(y, t)$. This is an enhancement to the current formulation of the single-point BHR model equations (Besnard, et al. 1987). Another term that enhances the BHR model (Besnard, et al. 1987), as derived in Appendix B, is the densitymass-flux gradient source term to $b(y, t)$. 


\section{Appendix F. Numerical Schemes}

\section{F.1 Introduction}

Three different numerical codes have been developed to obtain numerical results for the investigations of this report. The first code is written for the single-point turbulence transport model that is discussed in Chapter 3. This code transports the mean flow variables and the three single-point turbulence variables of the BHR turbulence transport model, $R_{i j}$, $a_{i}$, and b. A second code is written for the two-fluid model that is discussed in Chapter 4. This second code transports the mean flow variables, velocity $u_{i}$ and volume fraction $\alpha$, for each field as well as a mean pressure, p. There are no turbulent variables transported in this code. A third code is written for solving the spectral turbulence transport equations that are presented in Chapter 5. The third code transports the mean flow variables and the spectral turbulence variables $R_{i j}$, $a_{i}$, and $b$. Due to the spectral nature of the formulation, the third code is computationally more costly than the other two due to the added spectral dimension. All three codes incorporate an Eulerian mesh, are first order in both time and space, and due to the fact that the fluctuations of the flow are assumed to be far subsonic, are implicitly formulated to satisfy incompressibility.

For this study, the mixing layer is assumed to be infinite in the two spatial dimensions parallel to the original fluid interface, the $\mathrm{x}$ and $\mathrm{z}$ directions, and to have a finite width for the spatial dimension perpendicular to the original fluid interface, the y direction. Ensemble averaging of the fluctuating quantities is accomplished by performing strip averages on the infinite mixing layer in the $\mathrm{x}$ and $\mathrm{z}$ directions. The mean flow variables of the mixing layer are assumed to as statistically homogeneous in both the $\mathrm{x}$ and $\mathrm{z}$ directions, which restricts the variations to occur in only the one spatial direction, y. These assumptions allow for a onedimensional code that fully describes all of the three-dimensional physics of the mixing problem. For this problem, our numerical domain consists of a one-dimensional box composed of a column of cells with unit cross sectional area. The cells continue far enough 
past the initial fluid interface so as to allow the development of the TMZ to become selfsimilar.

For the one-dimensional configuration of this problem, the mean flow, $\overline{\mathbf{u}}$, is zero, i.e., there is no in flow or out flow at the upper or lower boundaries of the computational domain (box). From this constraint, Equation (2.2.9) becomes $\tilde{\mathbf{u}}=\mathbf{a}$. Equation (2.2.9) is substituted into the momentum equation and the time derivative of $\mathbf{a}$ is determined by the transport equation for $\mathbf{a}$. The resulting equation is then integrated over the entire physical domain in order to determine the pressure field, which in one dimension allows for solution of an implicit pressure field without the necessity of iteration. This technique works equally well for both the single-point code and the spectral code.

The code for the two-fluid model uses a similar procedure to solve for the pressure field. The momentum equations for the two fields are added, and the resulting equation is integrated to determine the pressure field. As in the case of the turbulence transport models, the addition of the momentum equations produces a time derivative term that must be closed. The addition of the time derivatives of the two momentum equations results in a time derivative of the mean flow velocity, i.e., $\alpha_{1} u_{1 i}+\alpha_{2} u_{2 i}=\bar{u}_{i}$; however $\overline{\mathbf{u}}=0$ at the boundaries of the box and is zero throughout the entire box due to incompressibility. Thus the pressure field can be determined. Likewise, the field can be determined implicitly without iterating due to the fact that the domain is one-dimensional.

All numerical computations for this study where performed on two unclassified SGI Indigo II workstations (Gnarly and Guido) on the LAN in Group T-3 at Los Alamos National Laboratory. All of the numerical code editing and post-processing of the data were performed on a Macintosh IIci.

\section{F.2 Solution procedure for the single-point turbulence transport equations}

The problem is initialized with pure heavy fluid $(\rho=2)$ in the top half of the column of cells and pure lighter fluid $(\rho=1)$ in the bottom half of the column of cells. However, 
there is one cell at the fluid interface that contains a mixture of half of each fluid. In this cell we also initialize $b$ with its maximum value, i.e. for a mixture of half of each fluid of the given densities, $b=0.125$. We realize that these are somewhat fictitious initial conditions, but these conditions bootstrap the calculation by initializing the source term to the aequation, i.e., the $b(d p / d x)$ term.

The code is first order in both time and the one spatial dimension. Upwind differencing is used in the momentum and mass equations. The boundary conditions at the top and bottom of the grid are insignificant due to the fact that the grid is made large enough to contain the developing TMZ to a state of self-similarity. Typically, this process required a grid of about 200 cells.

The logic flow chart used to solve the single-point turbulence transport equations (Eqs. (3.3.4) - (3.3.15) ) from Chapter 3 is as follows:

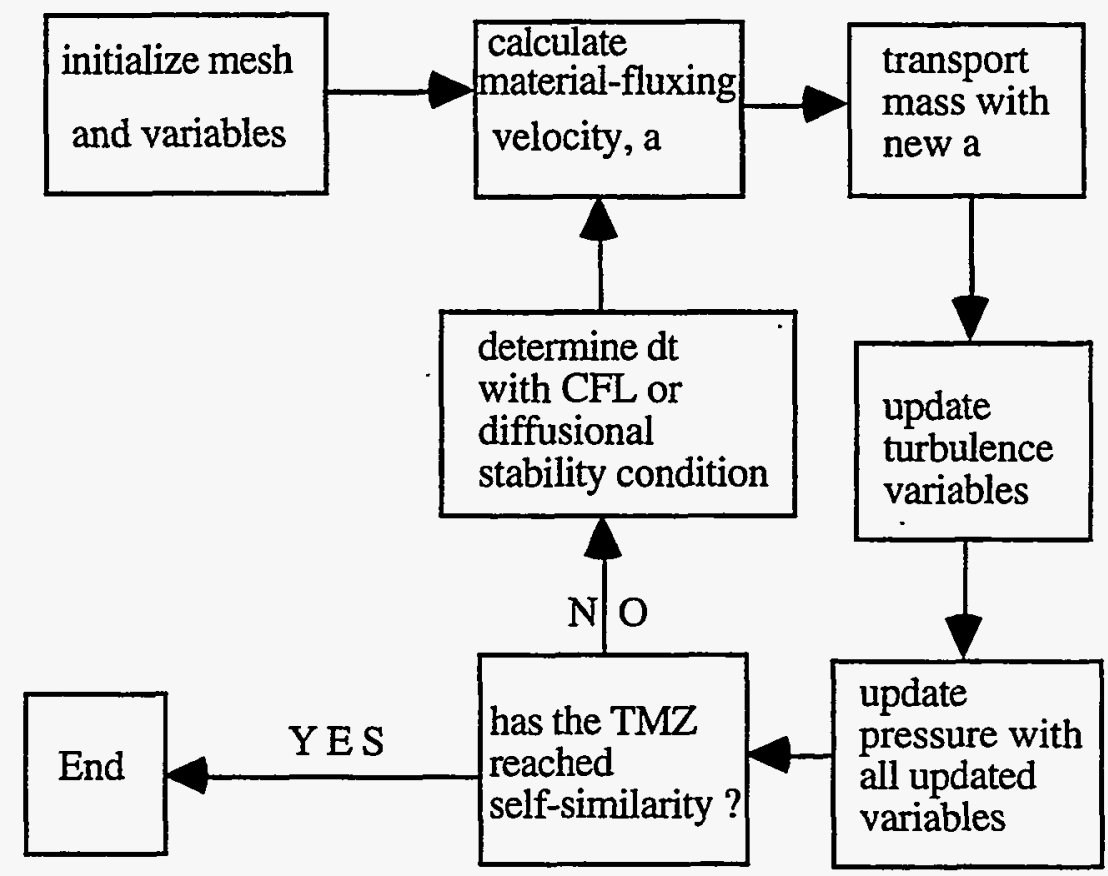

Figure F.1. Logic scheme for the numerical solution of the BHR single-point turbulence transport model.

The code for the numerical solution of the single-point turbulence transport equation of the BHR model is included in the following text. Only those subroutines involved with the 
variable updating process are included for brevity. All subroutines associated with $\mathrm{V} / \mathrm{O}$ or the formatting of $\mathrm{I} O \mathrm{O}$ have not been included.

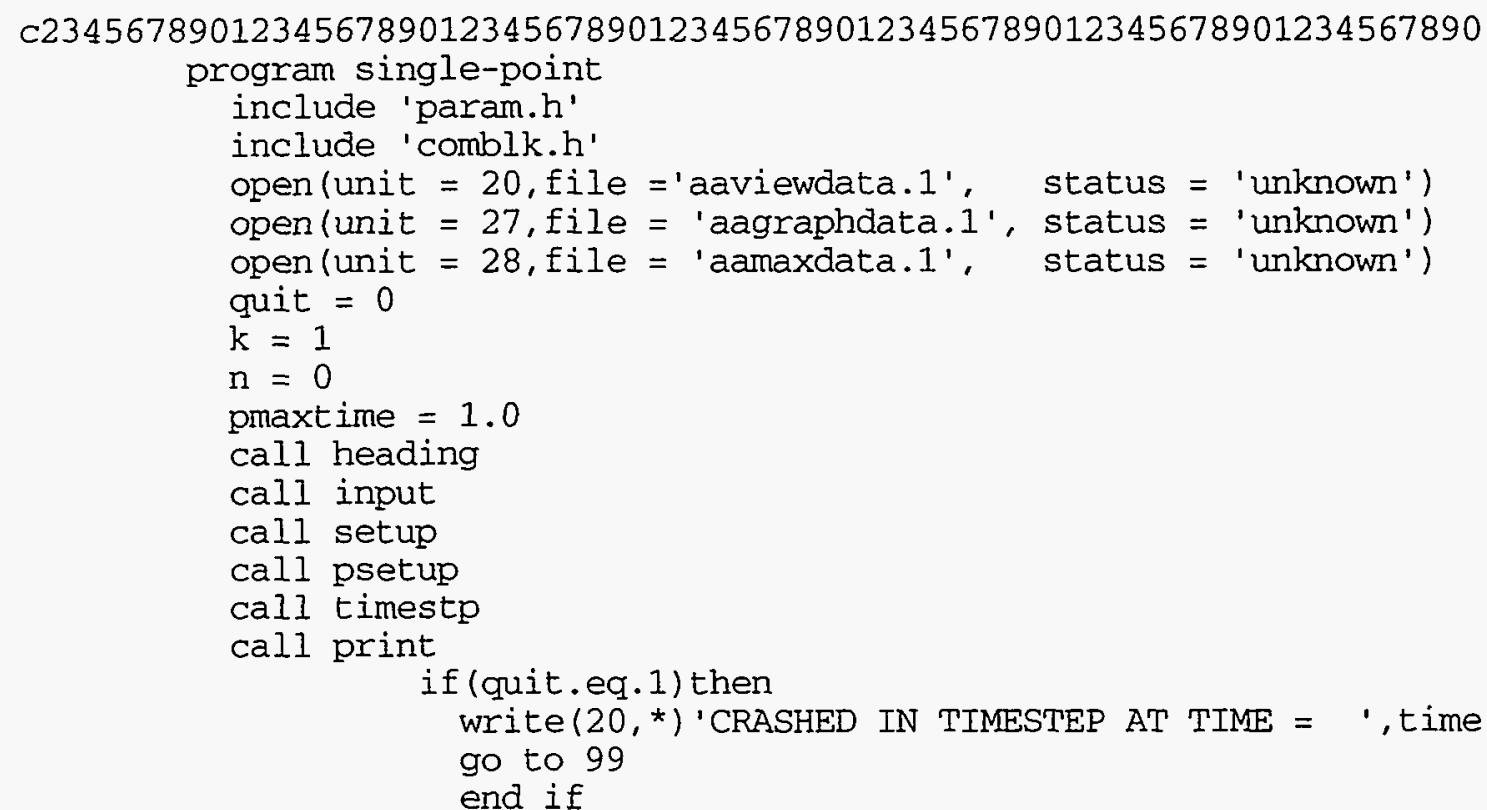

call grapher

call maxvalu

time $=\mathrm{dt}$$$
\text { write }\left(*,{ }^{*}\right) \text { 'time }={ }^{\prime} \text {, time,' } d t=', d t, ' n=', n
$$

ptime=dmptime $(\mathrm{k})$

do 20 while (time.1e. (runtime+1.1*dt))

if (time.ge.chvtopt) $\mathrm{g}=\mathrm{g} 2$

$\mathrm{n}=\mathrm{n}+1$

if (time.ge.pmaxtime) then

call maxvalu

pmaxtime $=$ pmaxtime +1.0

end if

call pbox

call abox

call massbox

call turbflo

if (time.ge.ptime) then

call print

call grapher

write $(*, *)$ 'time $=^{\prime}$, time,' $d t=^{\prime}, d t,{ }^{\prime} n={ }^{\prime}, n$

if (time.ge datadmp $(k+1))$ then

ptime $=$ datadmp $(k+1)$

end if

$\mathrm{k}=\mathrm{k}+1$

end if

ptime=pt ime+dmptime $(\mathrm{k})$

call timestp

time = time $+d t$

if (quit.eq. 1) then

write $(20, *)$ 'CRASHED IN TIMESTEP AT TIME $=1$, time call print

goto 99 
end if

20 continue

write $(20, *)$ ' '

99 continue

write $(20, *)$ 'RUN WAS SUCCESSFUL'

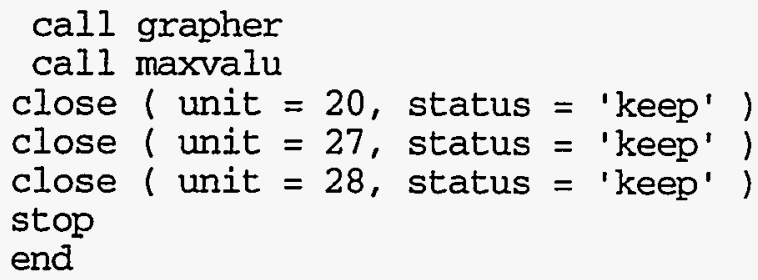




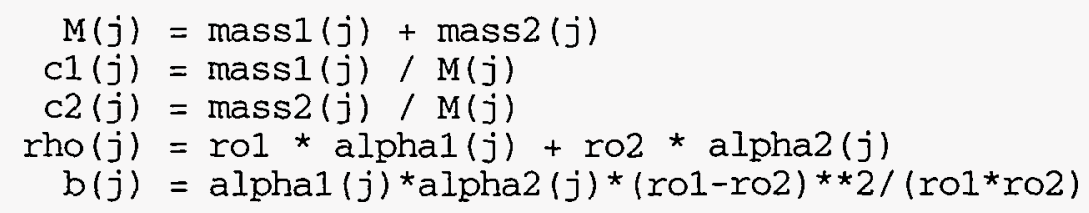

else

endif

$$
\begin{aligned}
M(j) & =r o 2 * d y \\
\operatorname{alpha1}(j) & =0.0 \\
\operatorname{alpha2}(j) & =1.0 \\
\operatorname{mass} 1(j) & =0.0 \\
\operatorname{mass} 2(j) & =M(j) \\
c 1(j) & =0.0 \\
c 2(j) & =1.0 \\
\operatorname{rho}(j) & =\text { ro2 }
\end{aligned}
$$

$y(j+1)=y(j)+d y$

$$
\begin{aligned}
\operatorname{tke}(j) & =\text { tkeo } \\
s(j) & =s 0
\end{aligned}
$$$$
\operatorname{tnu}(j)=0.09 * s(j) * \operatorname{sqrt}(\operatorname{abs}(t k e(j)))
$$

10 continue

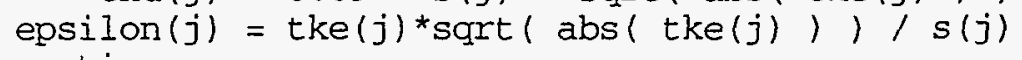




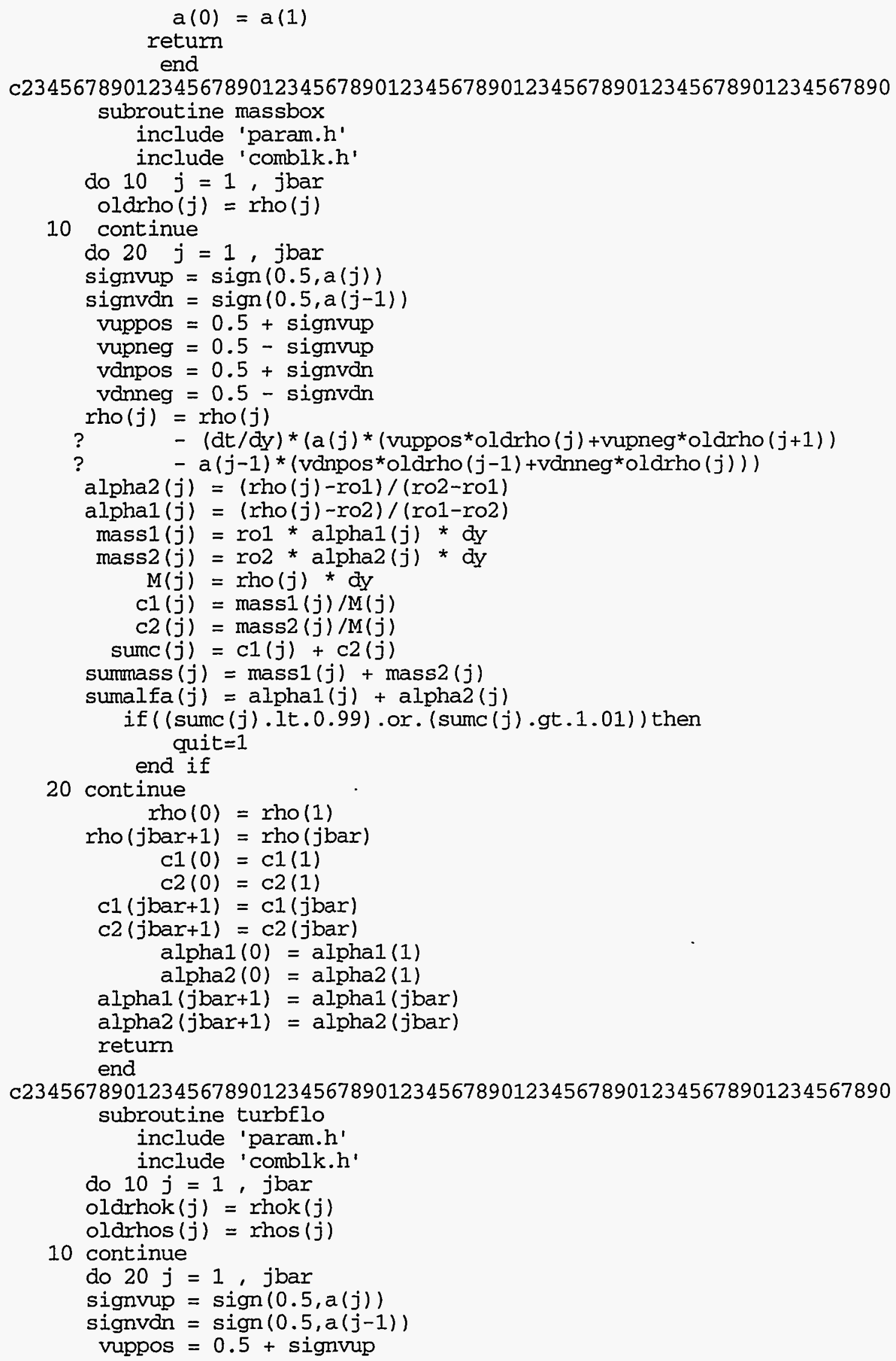




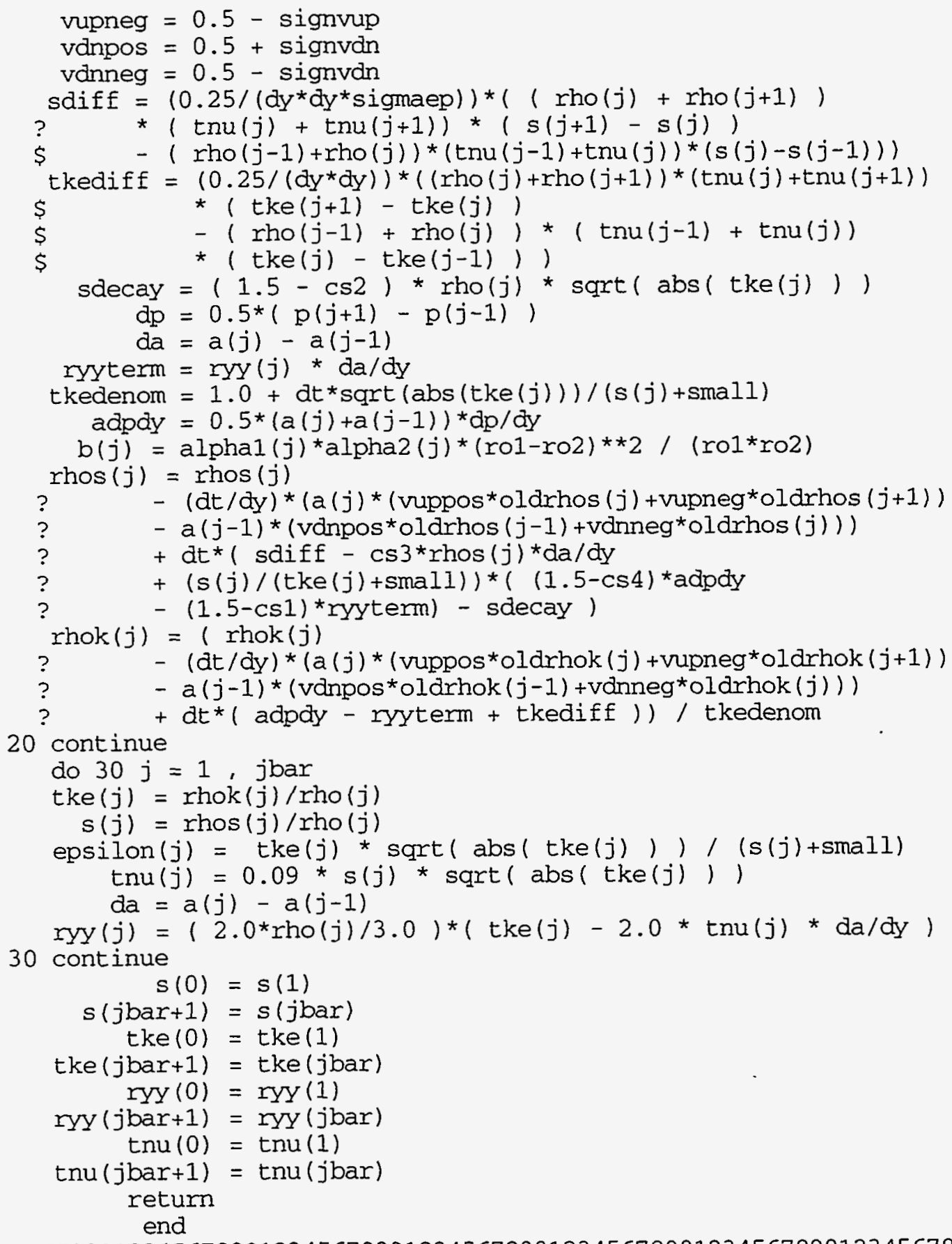




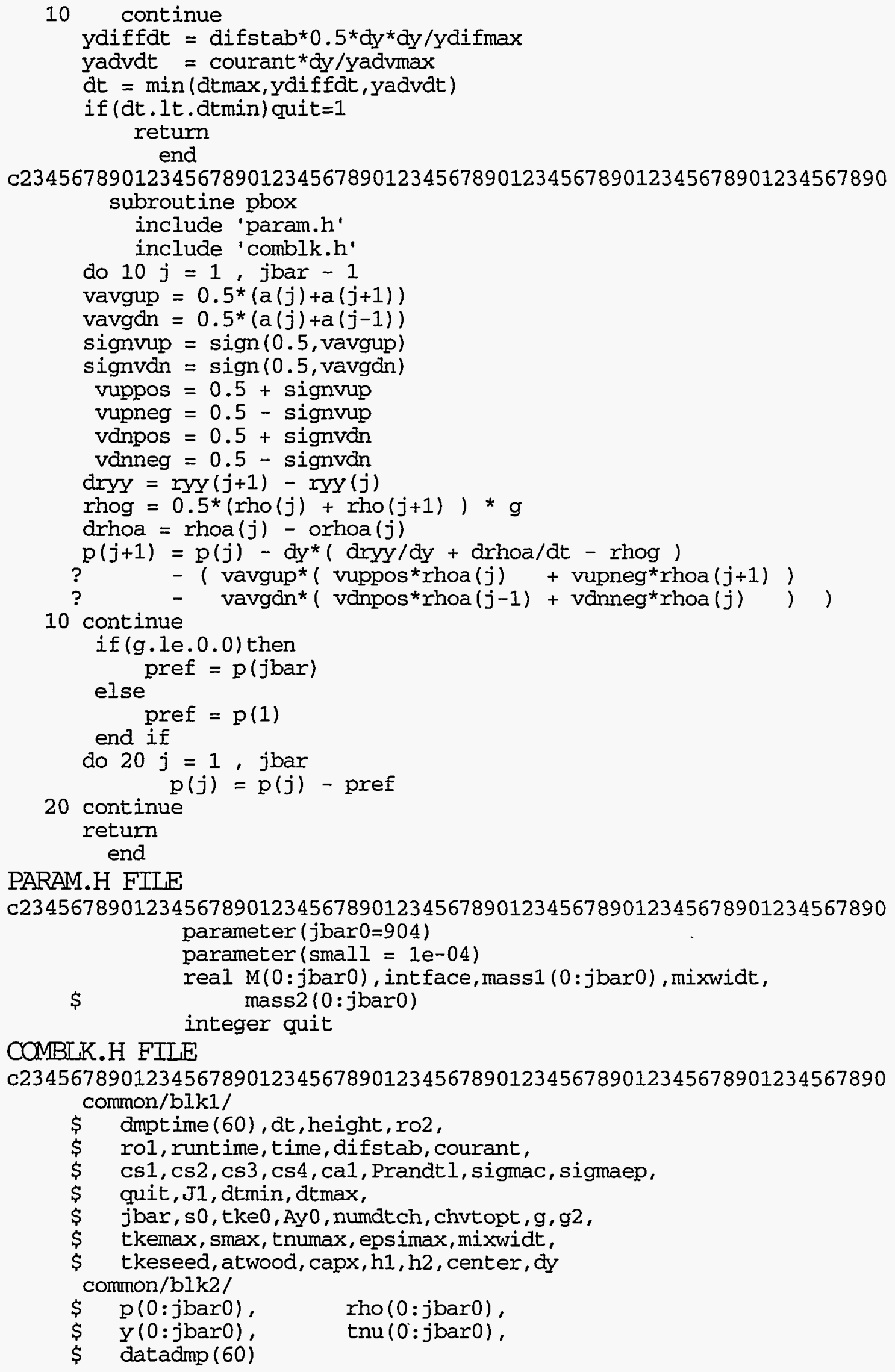




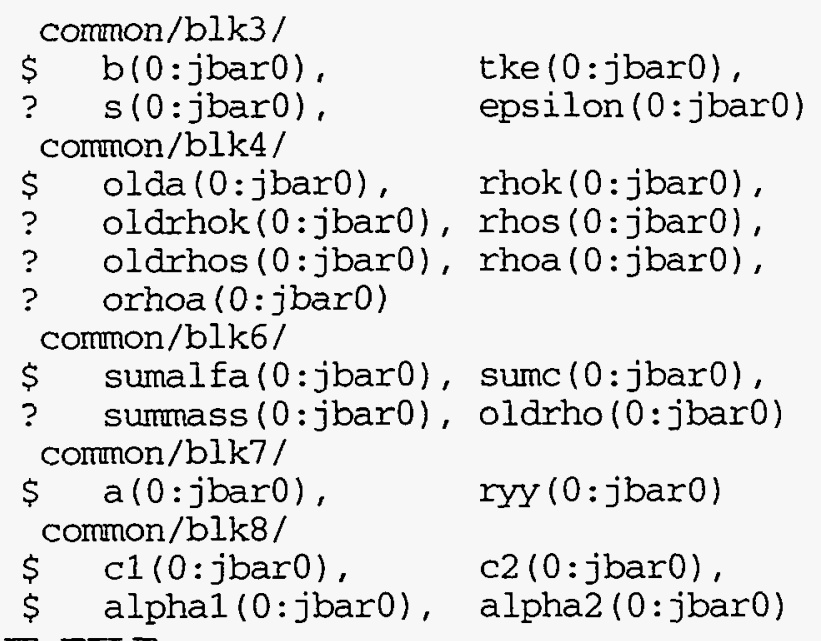

INPUT FIIE

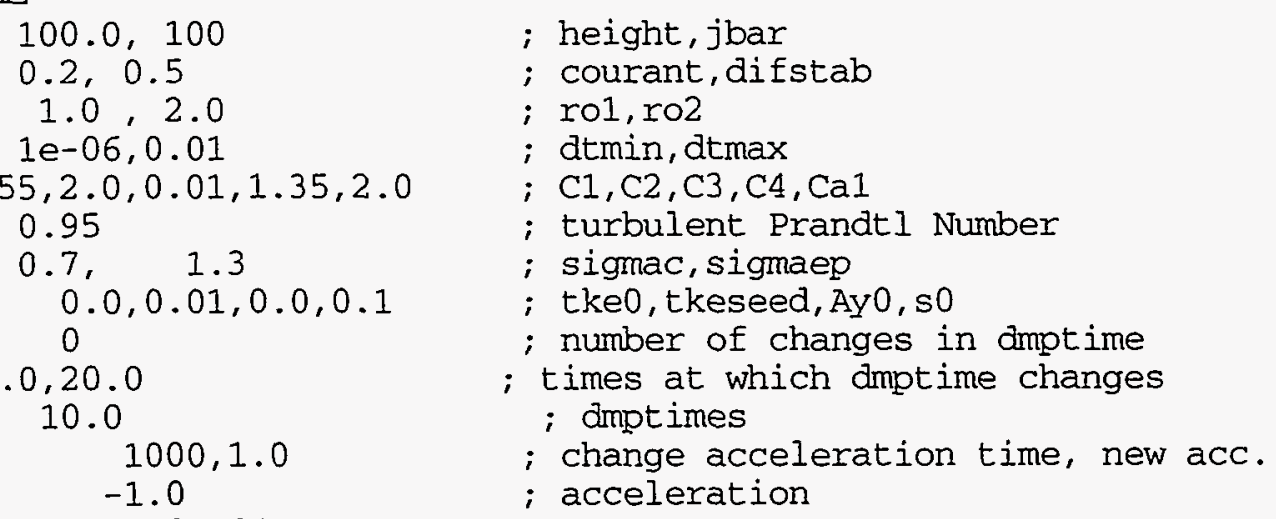

CODE VERSION: 04/17/95(1)

RUN DATE: 04/18/95

\section{F.3 Solution procedure for the two-field transport equations}

This numerical scheme transports the volume fractions, $\alpha$, and the velocities, $u$, of both fields. Conservation of mass constrains the relationship between volume fractions to be $\alpha_{1}+\alpha_{2}=1$. Small deviations away from this relation occur throughout the course of the calculation, and a corrective procedure which is suggested by Cook \& Harlow (1984b) is used to keep this sum close to unity. The converging iteration procedure they suggest to maintain this relation is outlined here. Let

$$
\bar{\lambda}_{0}=\frac{C_{R}(\delta y)^{2}}{2 \delta \mathrm{t}},
$$

where $C_{R}$ is set equal to 0.9 ,

$$
\left(\bar{\lambda}_{1}\right)_{\mathrm{j} \pm 1 / 2}=\frac{\bar{\lambda}_{0}}{2}\left[\left(\alpha_{1}\right)_{\mathrm{j}}+\left(\alpha_{1}\right)_{\mathrm{j} \pm 1}\right],
$$




$$
\left(\bar{\lambda}_{2}\right)_{\mathrm{j} \pm 1 / 2}=\frac{\bar{\lambda}_{0}}{2}\left[\left(\alpha_{2}\right)_{\mathrm{j}}+\left(\alpha_{2}\right)_{\mathrm{j} \pm 1}\right]
$$

$\left(\hat{\alpha}_{1}\right)_{\mathrm{j}}=\left(\alpha_{1}\right)_{\mathrm{j}}+\frac{\delta \mathrm{t}}{\delta \mathrm{x}^{2}}\left\{\left(\bar{\lambda}_{1}\right)_{\mathrm{j}+1 / 2}\left[\left(\alpha_{1}+\alpha_{2}\right)_{\mathrm{j}+1}-\left(\alpha_{1}+\alpha_{2}\right)_{\mathrm{j}}\right]-\left(\bar{\lambda}_{1}\right)_{\mathrm{j}-1 / 2}\left[\left(\alpha_{1}+\alpha_{2}\right)_{\mathrm{j}}-\left(\alpha_{1}+\alpha_{2}\right)_{\mathrm{j}-1}\right]\right\}$,

and

$\left(\hat{\alpha}_{2}\right)_{\mathrm{j}}=\left(\alpha_{2}\right)_{\mathrm{j}}+\frac{\delta \mathrm{t}}{\delta \mathrm{x}^{2}}\left\{\left(\bar{\lambda}_{2}\right)_{\mathrm{j}+1 / 2}\left[\left(\alpha_{1}+\alpha_{2}\right)_{\mathrm{j}+1}-\left(\alpha_{1}+\alpha_{2}\right)_{\mathrm{j}}\right]-\left(\bar{\lambda}_{2}\right)_{\mathrm{j}-1 / 2}\left[\left(\alpha_{1}+\alpha_{2}\right)_{\mathrm{j}}-\left(\alpha_{1}+\alpha_{2}\right)_{\mathrm{j}-1}\right]\right\}$.

$\bar{\lambda}_{1}$ and $\bar{\lambda}_{2}$ are set equal to zero at the rigid boundaries to ensure conservation. This system is solved by iteration (replacing $\alpha$ by $\hat{\alpha}$ at the end of each cycle) until the sum of $\alpha_{1}$ and $\alpha_{2}$ is sufficiently close to unity. Cook \& Harlow suggest that the allowable tolerance is $0.05 \%$.

This problem is initialized by placing pure heavy fluid in the top half of the cells and pure light fluid in the bottom half of the cells. It is not necessary to initialize a cell at the fluid interface with half of each fluid as is done for the case of the single-field turbulence transport models in Appendix F.2. The acceleration is set to -1.0 at $t=0$ and mixing begins.

The logic flow chart used to solve the two-field transport equations (Eqs. (4.3.1) (4.3.7) ) from Chapter 4 is as follows:

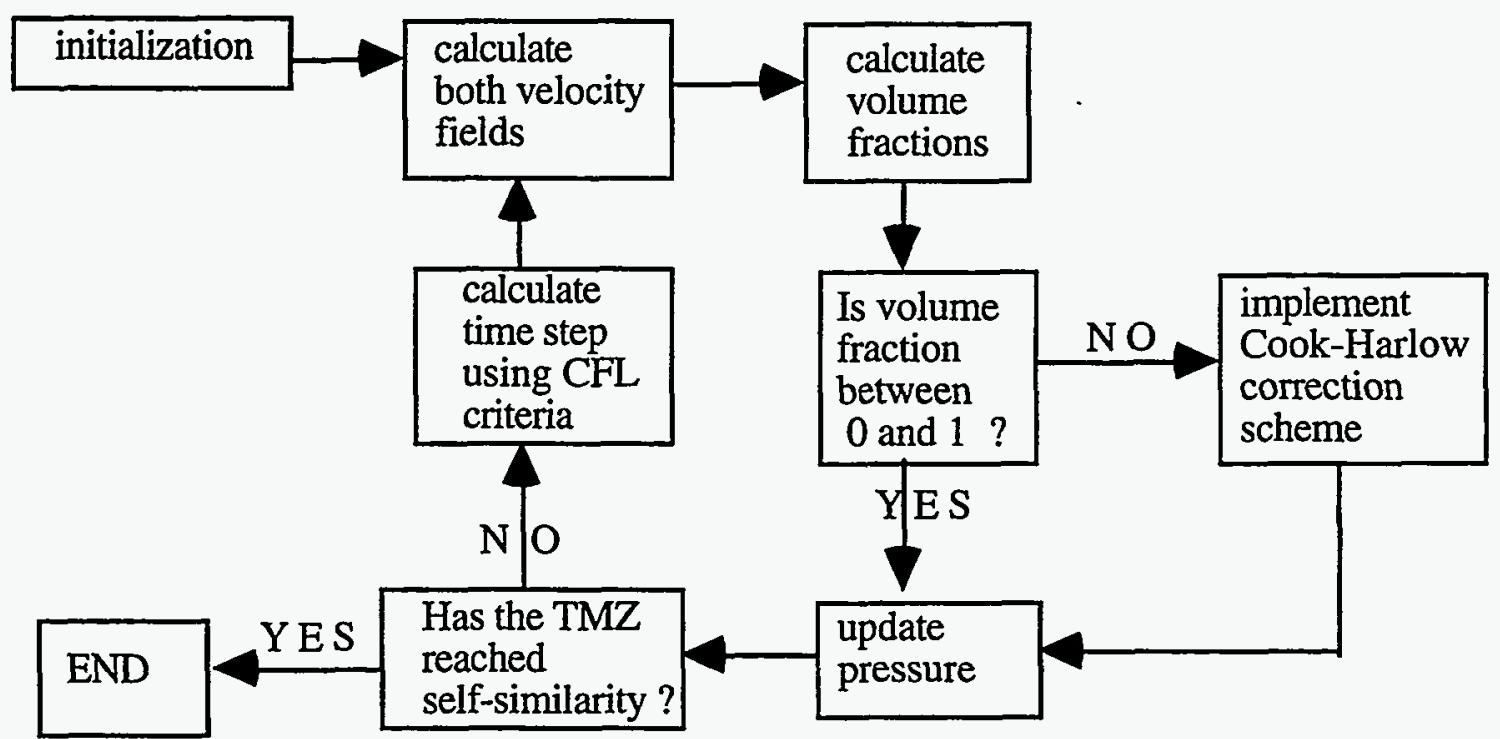

Figure F.2. Logic scheme for the numerical solution of the two-field transport model. 
The code for the numerical solution of the two-field transport model is included in the following text. Only those subroutines involved with the variable updating process are included for brevity. All subroutines associated with $\mathrm{I} / \mathrm{O}$ or the formatting of $\mathrm{I} / \mathrm{O}$ have not been included.

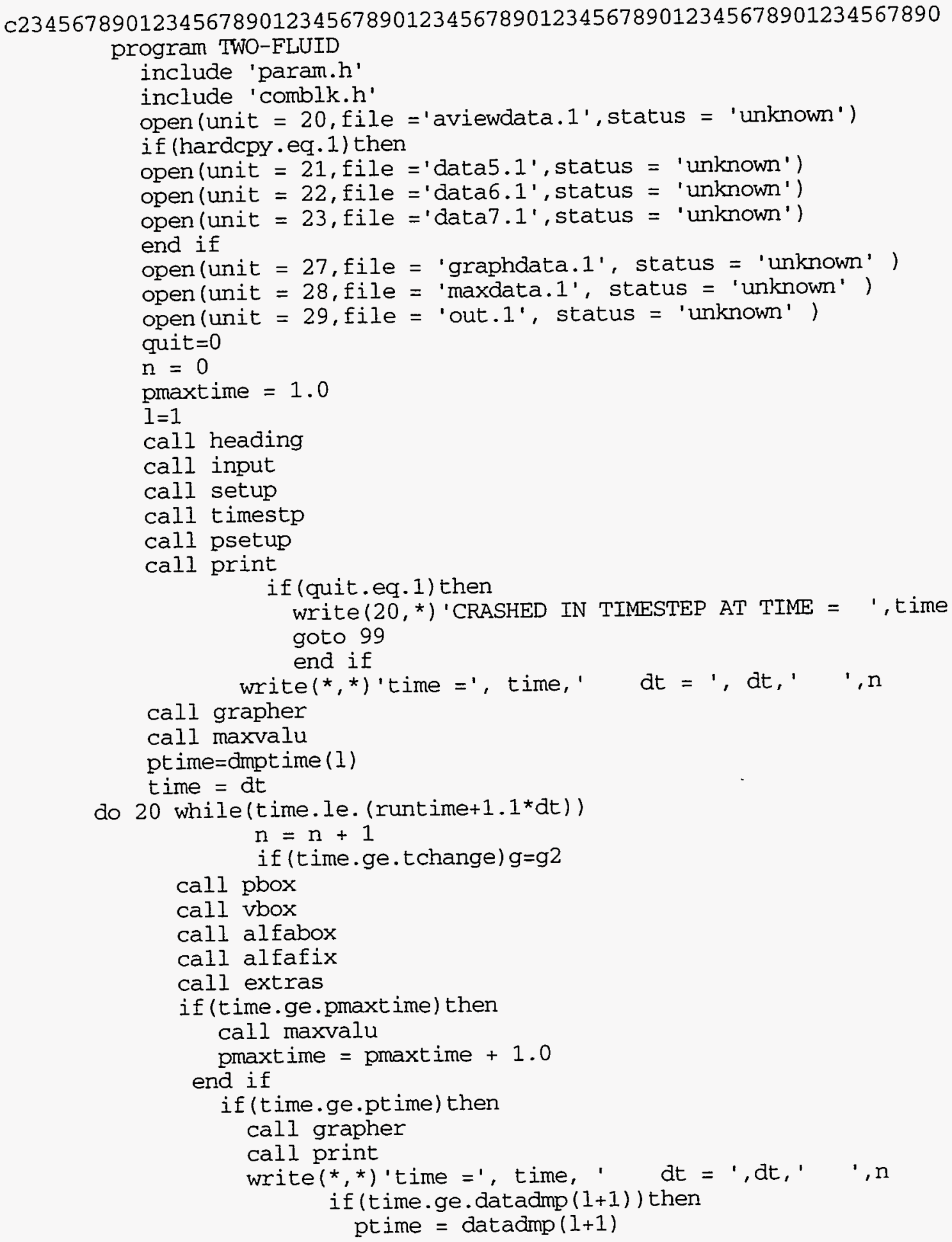




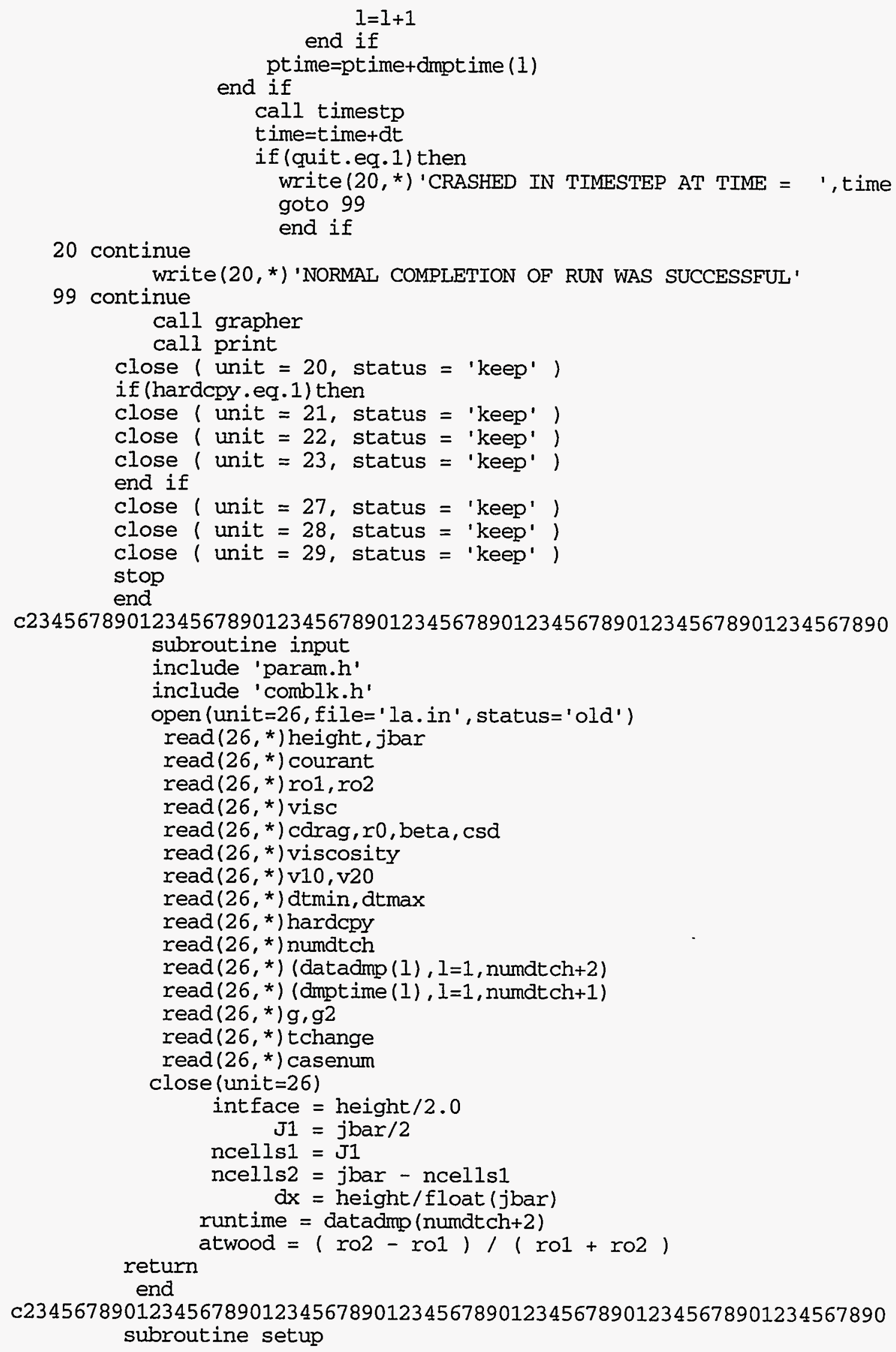


include 'param.h'

include 'comblk.h'

$\operatorname{vbar}(0)=0.0$

$y(0)=-0.5^{\star} d x$

$y$ cnorm $(0)=(y(0))-$ height $/ 2.0$

center $=0.0$

$\mathrm{v}(0)=\operatorname{vbar}(0)$

$\mathrm{v} 1(0)=\operatorname{vbar}(0)$

do $10 j=1$, jbar

$\mathrm{v} 2(0)=\operatorname{vbar}(0)$

$$
\begin{aligned}
\operatorname{vbar}(j) & =0.0 \\
\operatorname{v1}(j) & =\operatorname{v10} \\
\operatorname{v} 2(j) & =\operatorname{v20} \\
\operatorname{v1}(j \text { bar }) & =\operatorname{vbar}(j \text { bar }) \\
\operatorname{v} 2(j \text { bar }) & =\operatorname{vbar}(j \text { bar })
\end{aligned}
$$

if $(j . l e . J 1)$ then

$$
r h o(j)=\operatorname{rol}
$$

$M(j)=\operatorname{rho}(j) * d x$

$\operatorname{alpha1}(j)=1.0$

$\operatorname{alpha} 2(j)=0.0$

$\operatorname{mass} 1(j)=M(j)$

$\operatorname{mass} 2(j)=0.0$

$c 1(j)=1.0$

$c 2(j)=0.0$

else

endif

$$
\begin{aligned}
& r h o(j)=r o 2 \\
& M(j)=r h o(j) * d x \\
& \text { alpha1 }(j)=0.0 \\
& \text { alpha2 }(j)=1.0 \\
& \operatorname{mass}(j)=0.0 \\
& \operatorname{mass} 2(j)=M(j) \\
& c 1(j)=0.0 \\
& c 2(j)=1.0
\end{aligned}
$$

10 continue

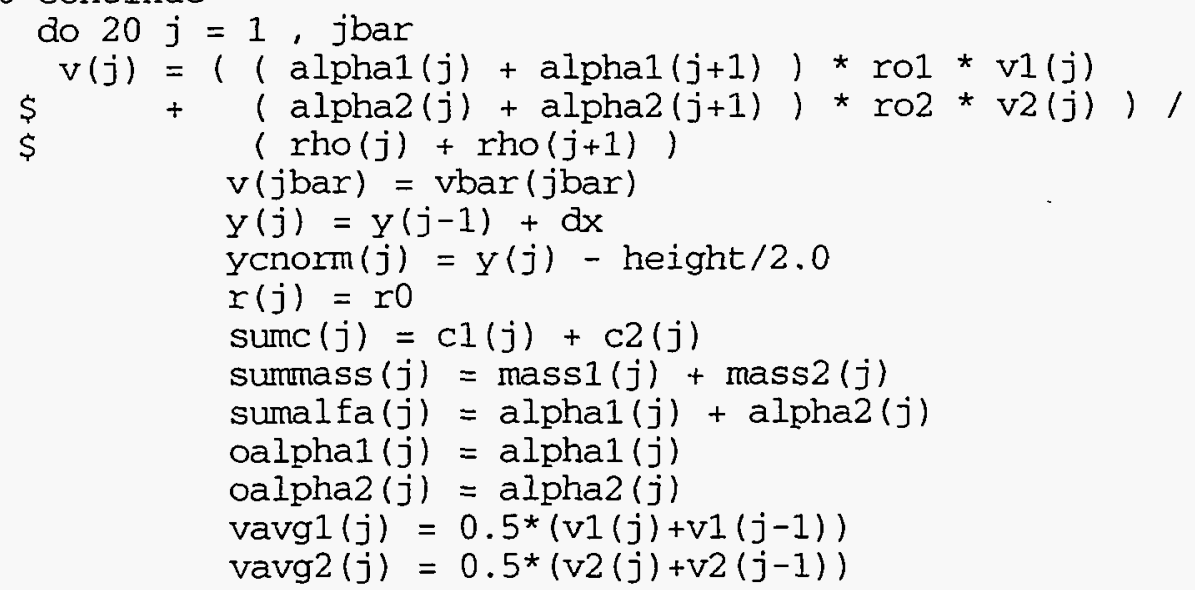

20 continue

$$
\begin{aligned}
& c 1(0)=c 1(1) \\
& c 1(j \text { bar }+1)=c 1 \text { (jbar) } \\
& c 2(0)=c 2(1) \\
& c 2(j \text { bar }+1)=c 2(j \text { bar }) \\
& r(0)=r 0=r 0 \\
& r(j \text { bar }+1)=r 0 \\
& \text { alpha1 }(0)=\text { alphal }(1)
\end{aligned}
$$


alpha2 $(0)=\operatorname{alpha2}(1)$

alpha1 $(j$ bar +1$)=$ alpha1 $(j$ bar $)$

alpha2 $(j b a r+1)=\operatorname{alpha2}(j$ bar $)$

oalphal $(0)=$ alphal $(0)$

oalphal $($ jbar +1$)=$ alphal $(j$ bar +1$)$

oalpha2 $(0)=$ alpha2 (1)

oalpha2 $(j$ bar +1$)=$ alpha2 $(j$ bar +1$)$

rho $(j$ bar +1$)=$ rho $(j$ bar $)$

return

rho $(0)=$ rho(1)

end

c234567890123456789012345678901234567890123456789012345678901234567890

subroutine vbox

include 'param.h'

include 'comblk.h'

do $10 j=0$, jbar

oalfaul $(j)=\operatorname{alfaul}(j)$

10 continue

oalfau2 $(j)=\operatorname{alfau} 2(j)$

do $20 j=1$, jbar -1

$\operatorname{signvup1}=\operatorname{sign}(0.5, \operatorname{vavg} 1(j+1))$

signvdn1 $=\operatorname{sign}(0.5, \operatorname{vavg} 1(j))$

vuppos $1=0.5+$ signvup 1

vupneg1 $=0.5-$ signvup1

vdnpos $1=0.5+$ signvdn 1

vdnneg1 $=0.5-$ signvan1

signvup2 $=\operatorname{sign}(0.5, \operatorname{vavg} 2(j+1))$

$\operatorname{signvdn2}=\operatorname{sign}(0.5, \operatorname{vavg} 2(j))$

vuppos2 $=0.5+$ signvup2

vupneg2 $=0.5-$ signvup2

vdnpos $2=0.5+$ signvdn2

vdnneg2 $=0.5-$ signvdn 2

galpha1 $=g * 0.5 *(a l p h a 1(j)+\operatorname{alpha1}(j+1))$

galpha2 $=g * 0.5 *(a l p h a 2(j)+a l p h a 2(j+1))$

alfrol $=0.5^{*}(\operatorname{alphal}(j)+\operatorname{alphal}(j+1)) / \mathrm{rol}$

alfro2 $=0.5^{*}(\operatorname{alpha} 2(j)+\operatorname{alpha} 2(j+1)) / \mathrm{ro} 2$

$d p=p(j+1)-p(j)$

$d v=v 2(j)-v 1(j)$

roalfa $=0.125 *($ rho $(j)+r h o(j+1))$

? * (alpha1 $(j)+\operatorname{alphal}(j+1)) *(\operatorname{alpha} 2(j)+\operatorname{alpha} 2(j+1))$

scale $=0.5 *(r(j)+r(j+1))$

momxchg $=0.375 * d v *$ roalfa

? * (cdrag* abs $(\mathrm{dv})+12.0 *$ visc / scale ) / scale

$\operatorname{alfaul}(j)=\operatorname{oalfaul}(j)$

? $\quad-(d t / d x) *(\operatorname{vavgl}(j+1) *(\quad$ vuppos1 * oalfaul $(j)$

? $\quad+\operatorname{vupnegl~*~oalfaul~}(j+1))$

? $-\operatorname{vavg} 1(j) *(\operatorname{vanpos} 1$ * oalfaul $(j-1)$

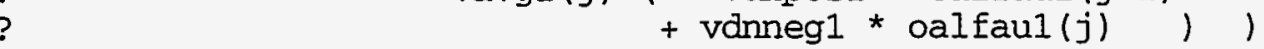

? $\quad+d t *(-a l f r o 1 * d p / d x+$ momxchg/ro1 + galpha1 $)$

$\operatorname{alfau} 2(j)=\operatorname{oalfau} 2(j)$

? $\quad-(d t / d x) *(\operatorname{vavg} 2(j+1) *(\quad \operatorname{vuppos} 2 *$ oalfau2 $(j)$

? $\quad+\operatorname{vupneg} 2 *$ oalfau2 $(j+1)$ )

? $\quad-\operatorname{vavg} 2(j) *(\operatorname{vdnpos} 2 *$ oalfau2 $(j-1)$

+ vdnneg 2 * oalfau2 $(j), 1$

? $\quad+d t *(-a l f r o 2 * d p / d x-$ momxchg/ro2 + galpha2 $)$

20 continue

do $30 j=0$, jbar

$\operatorname{oldv} 1(j)=v 1(j)$ 
30 continue

$$
\operatorname{oldv} 2(j)=v 2(j)
$$

do $40 j=1$, jbar

denom1 $=0.5^{*}(\operatorname{alpha1}(j)+\operatorname{alpha1}(j+1))$

denom2 $=0.5 *(\operatorname{alpha} 2(j)+\operatorname{alpha} 2(j+1))$

$v 1(j)=\operatorname{alfau} 1(j) /($ denom $1+$ small $)$

$v 2(j)=\operatorname{alfau} 2(j) /($ denom $2+$ small $)$

40

$\operatorname{vavg} 1(j)=0.5 *(\mathrm{v} 1(j-1)+\mathrm{v} 1(j))$

$\operatorname{vavg} 2(j)=0.5 *(\mathrm{v} 2(j-1)+\mathrm{v} 2(j))$

continue

$$
\mathrm{v} 1(0)=\mathrm{v} 1(1)
$$

$\mathrm{v} 2(0)=\mathrm{v} 2(1)$

$\mathrm{v} 1(j$ bar $)=\mathrm{v} 1(\mathrm{jbar}-1)$

$\mathrm{v} 2(j$ bar $)=\mathrm{v} 2($ jbar -1$)$

$\operatorname{vavg} 1(0)=\operatorname{vavg} 1(1)$

$\operatorname{vavg} 2(0)=\operatorname{vavg} 2(1)$

$\operatorname{vavg} 1(j$ bar $)=\operatorname{vavg} 1(j$ bar -1$)$

$\operatorname{vavg} 2(j$ bar $)=\operatorname{vavg} 2(j$ bar -1$)$

alfaul $(0)=\operatorname{alfaul}(1)$

alfau2 $(0)=\operatorname{alfau} 2(1)$

alfaul $(j$ bar $)=\operatorname{alfaul}(j$ bar -1$)$

alfau2 $(j$ bar $)=$ alfau2 $(j$ bar -1$)$

$C * * \star * \star * * * \star$ CALCULATION OF LENGTH SCALE

old $r=r(0)$

do $50, j=1$, jbar

$r(j)=$ beta $*$ mixwidt

50 continue

$r(0)=r(1)$

$r(j$ bar +1$)=r(j$ bar $)$

return

end

c234567890123456789012345678901234567890123456789012345678901234567890

subroutine alfabox

include 'param.h'

include 'comblk.h'

do $10 j=0$, jbar +1

oalphal $(j)=\operatorname{alpha1}(j)$

10 continue

oalpha2 $(j)=\operatorname{alpha} 2(j)$

do $20 j=1$, jbar

$\operatorname{alpha1}(j)=\operatorname{oalpha1}(j)-(d t / d x) *(\operatorname{alfaul}(j)-\operatorname{alfaul}(j-1))$

?

?

$\operatorname{alpha} 2(j)=\operatorname{oalpha} 2(j)-(d t / d x) *(\operatorname{alfau} 2(j)-\operatorname{alfau} 2(j-1))$

? $\quad+\left(d t^{*}\right.$ viscosity $\left./\left(d x^{*} d x\right)\right) *(o a l p h a 2(j+1)$

? $\quad-2.0^{*}$ oalpha2 $\left.(j)+o a l p h a 2(j-1)\right)$

if (alphal (j).1t.0.0) then

$\operatorname{alpha1}(j)=0.0$

$\operatorname{alpha} 2(j)=1.0$

end if

if (alpha2 $(j) .1 t .0 .0)$ then

$\operatorname{alpha1}(j)=1.0$

end if

$\operatorname{alpha} 2(j)=0.0$

$\operatorname{sumalfa}(j)=\operatorname{alphal}(j)+\operatorname{alpha} 2(j)$

20 continue

$$
\begin{aligned}
& \text { alpha1 }(0)=\operatorname{alpha1}(1) \\
& \text { alpha2 }(0)=\operatorname{alpha2}(1)
\end{aligned}
$$




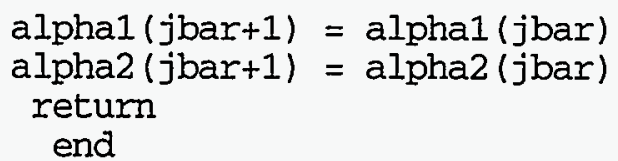


end

c234567890123456789012345678901234567890123456789012345678901234567890

subroutine pbox

include 'param.h'

include 'comblk. $h$ '

do $10 j=1$, jbar-1

signvup1 $=\operatorname{sign}(0.5, \operatorname{vavg} 1(j+1))$

signvdn1 $=\operatorname{sign}(0.5, \operatorname{vavg} 1(j))$

vuppos $1=0.5+$ signvup1

vupneg1 $=0.5-$ signvup1

vdnpos1 $=0.5+$ signvdn1

vdnneg1 $=0.5-$ signvdn 1

$\operatorname{signvup2}=\operatorname{sign}(0.5, \operatorname{vavg} 2(j+1))$

$\operatorname{signvdn} 2=\operatorname{sign}(0.5, \operatorname{vavg} 2(j))$

vuppos2 $=0.5+$ signvup2

vupneg2 $=0.5-$ signvup2

vdnpos $2=0.5+$ signvdn2

vanneg2 $=0.5-$ signvdn2

$x x=2.0^{*} d x^{*} r o 1 * r o 2 /(\operatorname{ro2} *(\operatorname{alpha1}(j)+\operatorname{alpha1}(j+1))$

? $\quad+\operatorname{ro1} *(\operatorname{alpha2}(j)+\operatorname{alpha} 2(j+1))$

$d v=(v 2(j)-v 1(j))$

roalfa $=0.125 *(\operatorname{rho}(j)+r h o(j+1))$

? * (alpha1 $(j)+$ alphal $(j+1)) *(\operatorname{alpha} 2(j)+\operatorname{alpha} 2(j+1))$

scale $=0.5^{*}(r(j)+r(j+1))$

momxchg $=(($ ro $1-\mathrm{ro} 2) /($ ro $1 *$ ro 2$)) * 0.375 * d v *$ roalfa

$\$ \quad *$ (cdrag * abs $(d v)+12.0 *$ visc / scale ) / scale $p(j+1)=p(j)-x x *($

$?(1.0 / d x) *(\operatorname{vavg} 1(j+1) *(\operatorname{vuppos} 1 * \operatorname{alfau} 1(j)+\operatorname{vupneg} 1 * \operatorname{alfau} 1(j+1))$

? $\quad-\operatorname{vavg} 1(j) *(\operatorname{vanpos} 1 * a l$ faul $(j-1)+\operatorname{vdnneg} 1 * a l f a u 1(j)))$

$?+(1.0 / d x) *(\operatorname{vavg} 2(j+1) *(\operatorname{vuppos} 2 * \operatorname{alfau} 2(j)+\operatorname{vupneg} 2 * \operatorname{alfau} 2(j+1))$

? - $\quad \operatorname{vavg} 2(j) *(\operatorname{vanpos} 2 * \operatorname{alfau} 2(j-1)+\operatorname{vanneg} 2 * \operatorname{alfau} 2(j)))$

? $\quad+$ momxchg $-\mathrm{g})$

10 continue

if $(g . l e .0 .0)$ then

pref $=p(j$ bar $)$

else

pref $=p(1)$

end if

do $20 j=1$, jbar

20 continue

$$
p(j)=p(j)-\text { pref }
$$

$p($ jbar +1$)=p($ jbar $)+0.5 * d x *($ rho $(j$ bar $)+r h o(j$ bar +1$)) * g$

return

$p(0)=p(1)-0.5 * d x *($ rho $(0)+r h o(1)) * g$

end

COMBLK.H FILE

C234567890123456789012345678901234567890123456789012345678901234567890

common/blk1/

$\$ d t$, height, rol, ro2, h1 , h2, cdrag, r $(0: j$ bar 0$), r 0$,

$\$$ runtime, time, courant, center,

$\$$ dtmin, dtmax, int face, beta, dx, g2, tchange, csd,

$\$$ mixwidt, atwood, capx, viscosity,

$\$$ casenum, bmax, g, v10, v20, visc, momxchg, sum

common/blk2/

$\$ p(0: j \operatorname{bar} 0)$, rho $(0: j \operatorname{bar} 0), \operatorname{vref}(0: j \operatorname{bar} 0), M(0: j \operatorname{bar} 0), v 2(0: j \operatorname{bar} 0)$,

$\$ \quad \mathrm{v} 1(0: j$ bar 0$), \mathrm{y}(0: j \operatorname{bar} 0), \mathrm{yc}(0:$ jbar 0$)$,

$\$$ datadmp (10),v(0:jbar0), dmptime (10), oldv1 $(0: j \operatorname{bar} 0)$,

$\$$ oldv2 $(0: j$ bar 0$)$, dalfaul $(0: j$ bar 0$)$, dalfau2 $(0: j$ bar 0$)$, 


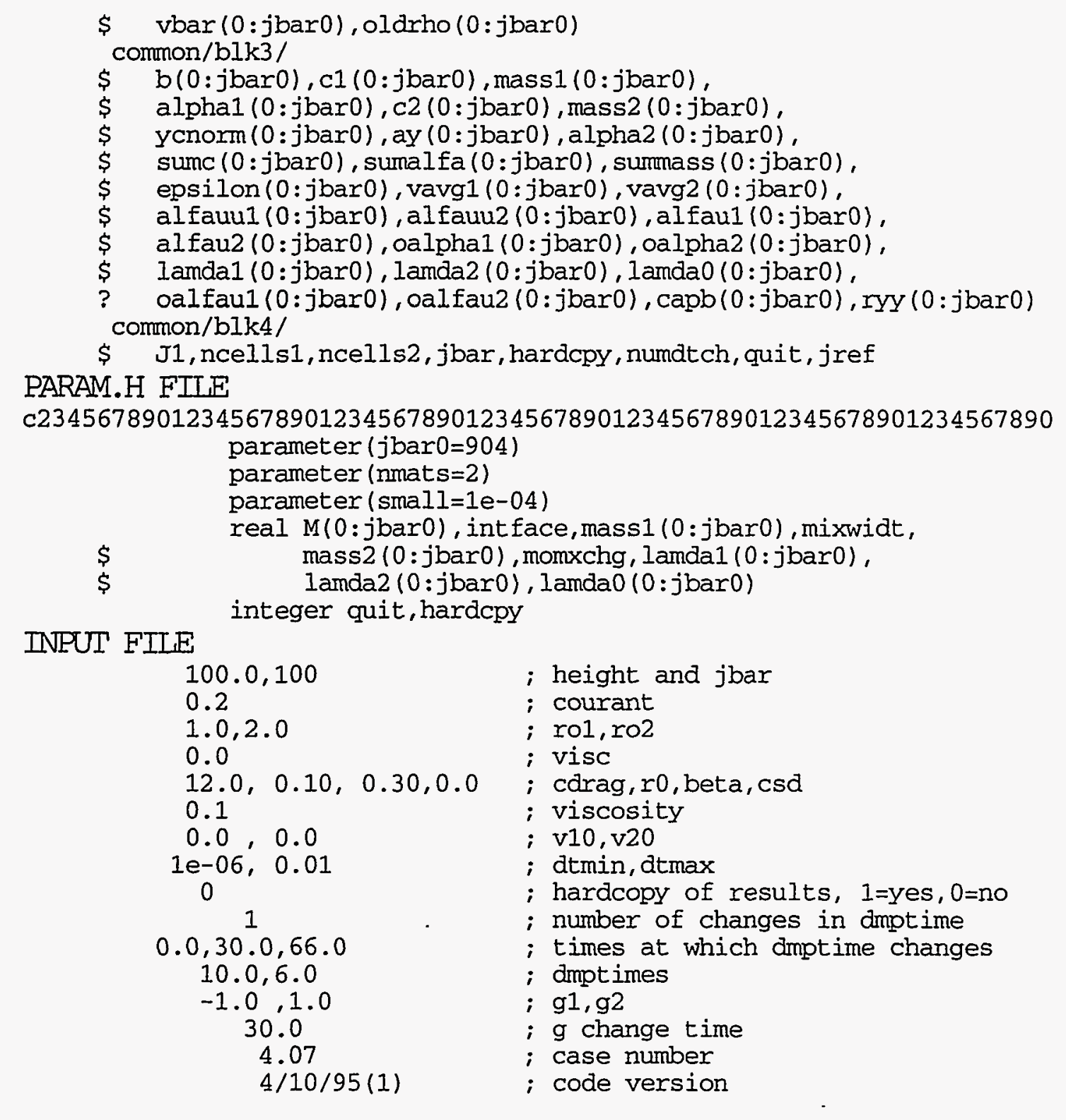

\section{F.4 Solution procedure for the spectral turbulence transport equations}

Since this code requires a two-dimensional grid, a much greater computational time is required. The equations are solved in y-space and logarithmic $k$-space (called $z$-space). This transformation from $\mathrm{k}$-space (as the equations are presented in the text) to $\mathrm{z}$-space is a convenience that allows for a spreading of the spectrum for ease of interpretation. The transformation is $\mathrm{z}=\mathrm{Ln}\left(\mathrm{k} / \mathrm{k}_{0}\right)$, where $\mathrm{k}_{0}$ is some constant wave number used to determine the position of the maximum of the initializing spectrum. For all cases discussed in this study, $\mathrm{k}_{0}$ 
is set equal to 1.0. The dependent variables used in the computations are $\mathrm{ka}_{\mathrm{i}}, \mathrm{kb}$, and $\mathrm{kR}_{\mathrm{ij}}$. All variables are transformed back to their original form, i.e., their $k$-space description, before any post processing is done.

The algorithm employs upwind differencing in $y$-space, and centered differencing in z-space. Upwind differencing was tried for z-space, but it made virtually no difference, so we returned to centered differencing for simplicity. The code is first-order accurate in time and both $z$ and $y$ space. A tridiaganol solver is used to solve the $z$-space direction of the computation implicitly. This is done, because, due to the fact that the advecting velocities in $\mathrm{z}$-space are exponential in $\mathrm{z}$, the explicit time step for large values of $\mathrm{z}$ (out at high wave numbers) is driven down to very small values. This allows for a time step that is larger than the explicit CFL condition or the diffusional stability condition and more acceptable running times.

As in the single-point code, the y-space boundary conditions are insignificant due to the fact that the spatial grid is made large enough and the problem is terminated before the $\mathrm{TMZ}$ reaches the edges of the grid. In $z$-space, a power law boundary condition is used for both the high and low wave numbers. A method was tried which allowed for the spectrum to find its own power law behavior at the edges of z-space (Clark, 1992), but numerical difficulties dictated that we use the same power law with which we initialized the bspectrum. The grid was made large enough in $z$-space such that the different power laws (within reason) where found to have virtually no effects on the dynamics of the spectrum.

The problem is initialized with a spectrum for $b$ in the cell that contains the interface of the two fluids. That is, qualitatively represent the perturbations that are present on the interface before the problem initiates. The $b$ spectrum follows a power law in $k$, i.e., $\mathrm{k}^{\mathrm{n}}$, at both the high and low wave numbers. At the high wave numbers, $n=-5 / 3$, and at the low wave numbers, $n=4$ or $n=2$ (numerical examples of both low wave number initializations are given in Chapter 7). The spectrum is normalized such that the integration of the spectrum 
over z-space yields the maximum value for configurational $b$ as discussed in Chapter 2 , and the maximum of the spectrum coincides with $\mathrm{k}=\mathrm{k}_{0}$, where $\mathrm{k}_{0}=1.0$.

The logic flow chart used to solve the two-point spectral transport equations (Eqs. (5.3.19), (5.3.26), (5.3.28), and (5.3.29) ) from Chapter 5 is as follows:

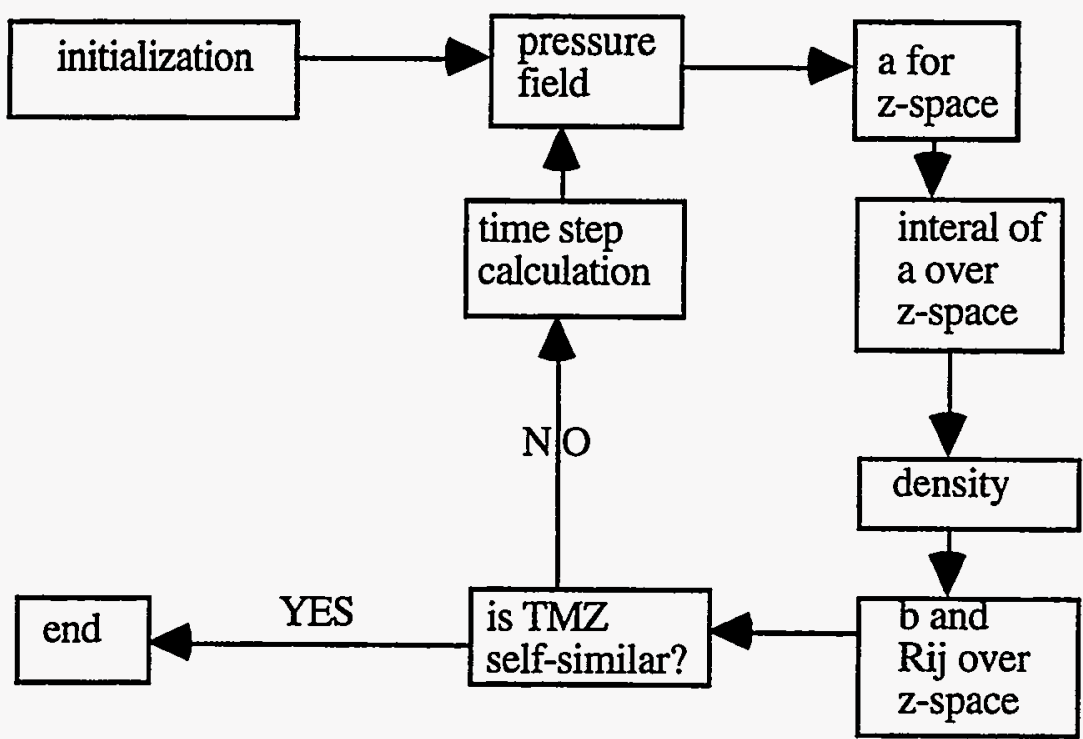

Figure F.3. Logic schematic for the numerical solution of the two-point spectral transport equations.

The code for the numerical solution of the two-point turbulence transport equations is included in the following text. Only those subroutines involved with the variable updating process are included for brevity. All subroutines associated with $\mathrm{I} / \mathrm{O}$ or the formatting of $\mathrm{I} / \mathrm{O}$ have not been included.

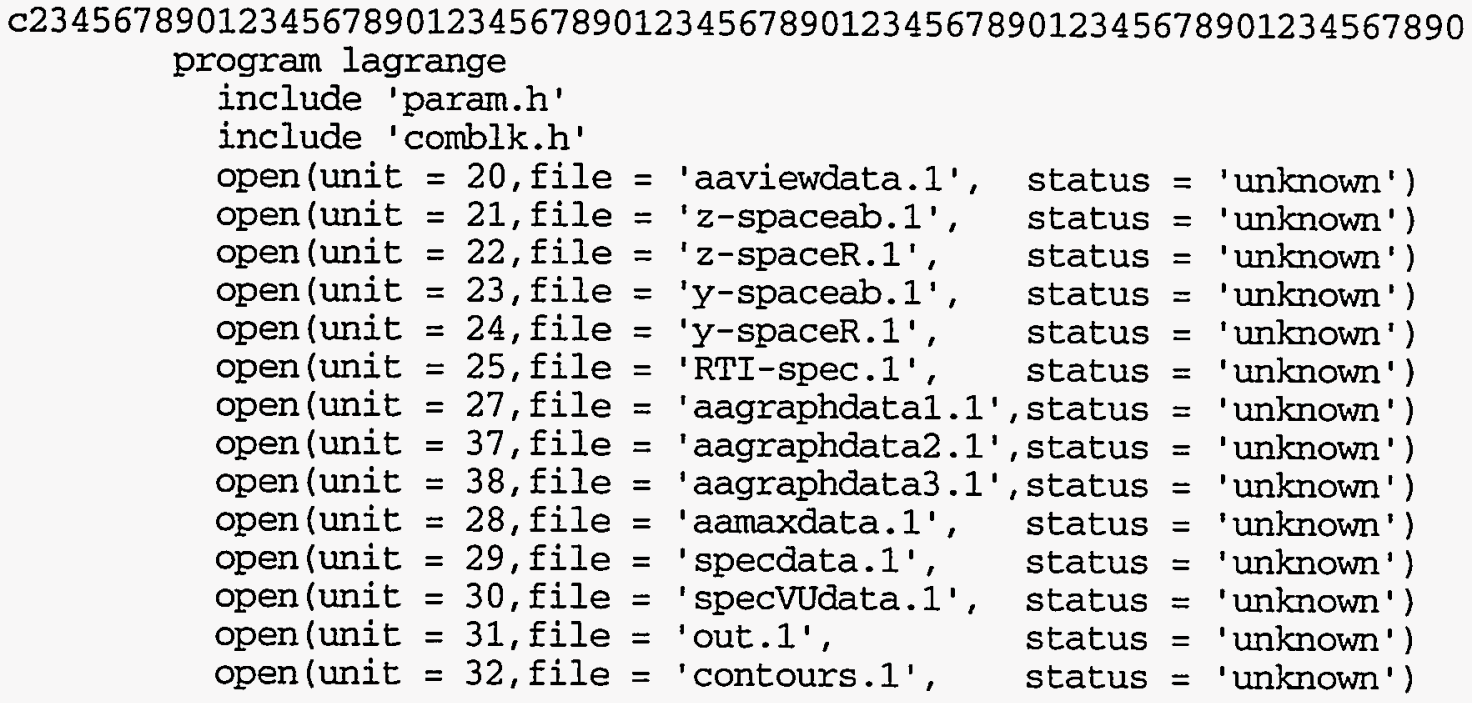




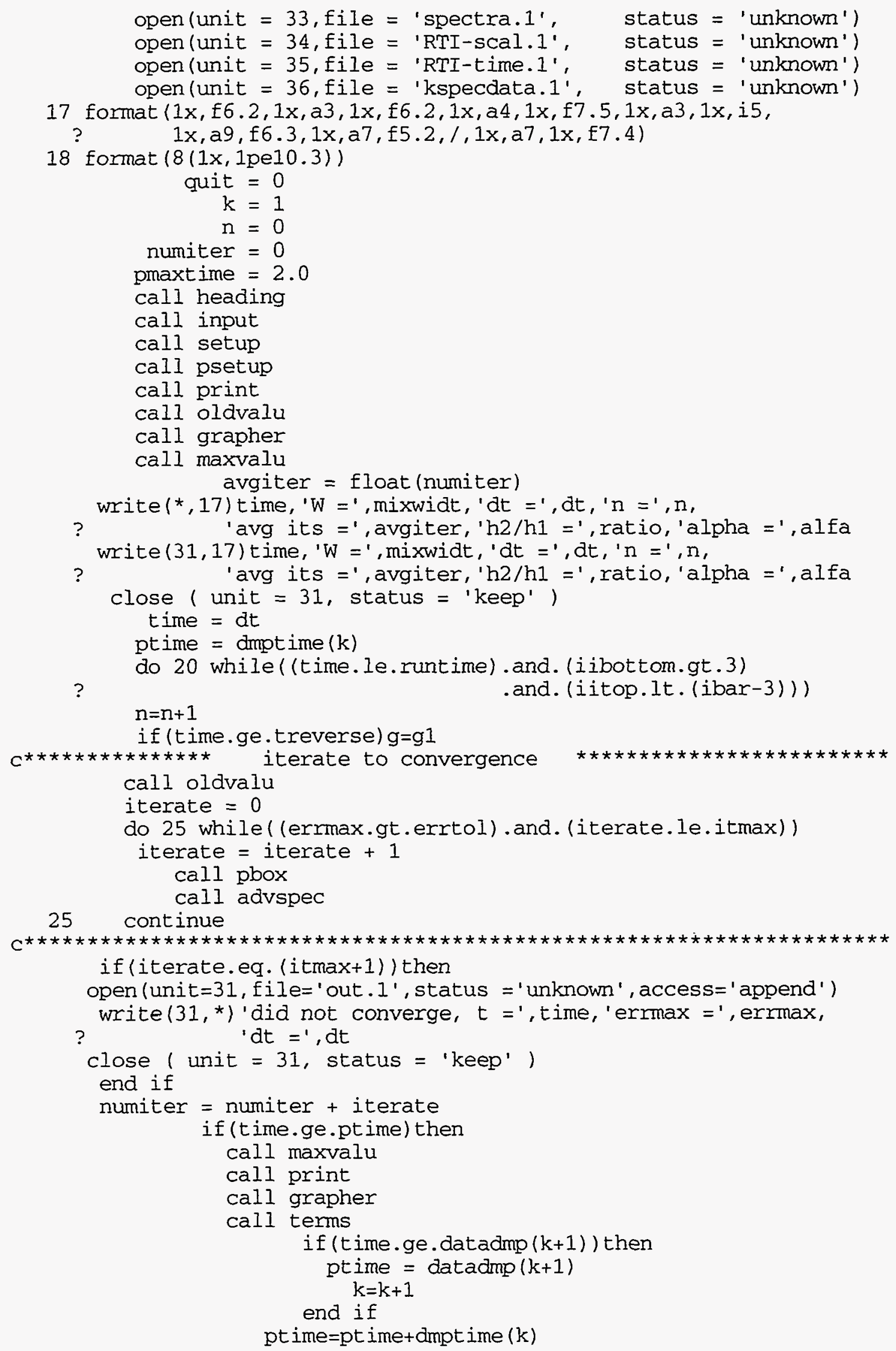


end if

if (time.ge.pmaxtime) then

call maxvalu

pmaxtime $=$ pmaxtime +1.0

avgiter $=$ float (numiter) $/$ float $(n)$

open (unit =31, file= 'out. 1 ' , status $=$ ' unknown' ' access = ' append' ')

write $(31,17)$ time, ' $W={ }^{\prime}$, mixwidt, ' $d t={ }^{\prime}, d t, ' n=', n$,

?

' avg its =', avgiter, ' $h 2 / \mathrm{h} 1=$ ', ratio, 'alpha =', alfa

close ( unit $=31$, status $=$ 'keep' )

end if

20 continue

$$
\begin{aligned}
& \text { call timestp } \\
& \text { time }=\text { time }+ \text { dt } \\
& \text { if (quit.eq.1) goto } 99
\end{aligned}
$$

call maxvalu

call print

call grapher

call terms

c WRITE TO CONTOURS

do $40 i=1$, ibar

do $50 \mathrm{j}=1$, jbar

write $(32,18) z(j), y c(i), \operatorname{time}, a(i, j), b(i, j), r(i, j), \operatorname{ryy}(i, j)$,

? $\operatorname{adpdy}(i, j)$

50 continue

40 continue

write $(20, *)$ ' '

write $(20, *)$ 'RUN WAS SUCCESSFUL'

write $(30, *) '$ '

write $(30, *)$ 'RUN WAS SUCCESSFUL'

avgiter $=$ float (numiter) $/$ float $(n)$

open (unit $=31$, file $=$ ' out. 1 ' , status $=$ ' unknown', access= ' append ' )

write $(31,17)$ time, ' $W={ }^{\prime}$, mixwidt, ' $d t={ }^{\prime}$, $d t,{ }^{\prime} n='$, $n$,

99

'avg its =', avgiter, 'h2/h1 =', ratio, 'alpha =', alfa write $(31, *)$ 'RUN WAS SUCCESSFUL'

continue

close ( unit $=20$, status $=$ 'keep' )

close ( unit $=21$, status $=$ 'keep' )

close ( unit $=22$, status $=$ 'keep')

close ( unit $=23$, status $=$ 'keep' )

close ( unit $=24$, status $=$ ' keep' )

close ( unit $=25$, status $=$ 'keep' )

close ( unit $=27$, status $=$ 'keep' )

close ( unit $=28$, status $=$ ' keep')

close ( unit $=29$, status $=$ ' keep' )

close ( unit $=30$, status $=$ ' keep' )

close ( unit $=31$, status $=$ ' keep' )

close ( unit $=32$, status $=$ 'keep')

close ( unit $=33$, status $=$ ' keep' )

close ( unit $=34$, status $=$ 'keep' )

close ( unit $=35$, status $=$ ' keep')

close ( unit $=36$, status $=$ 'keep')

close ( unit $=37$, status $=$ ' keep' )

close ( unit $=38$, status $=$ 'keep' )

stop

end

c234567890123456789012345678901234567890123456789012345678901234567890

subroutine input

include 'param. $h$ ' 


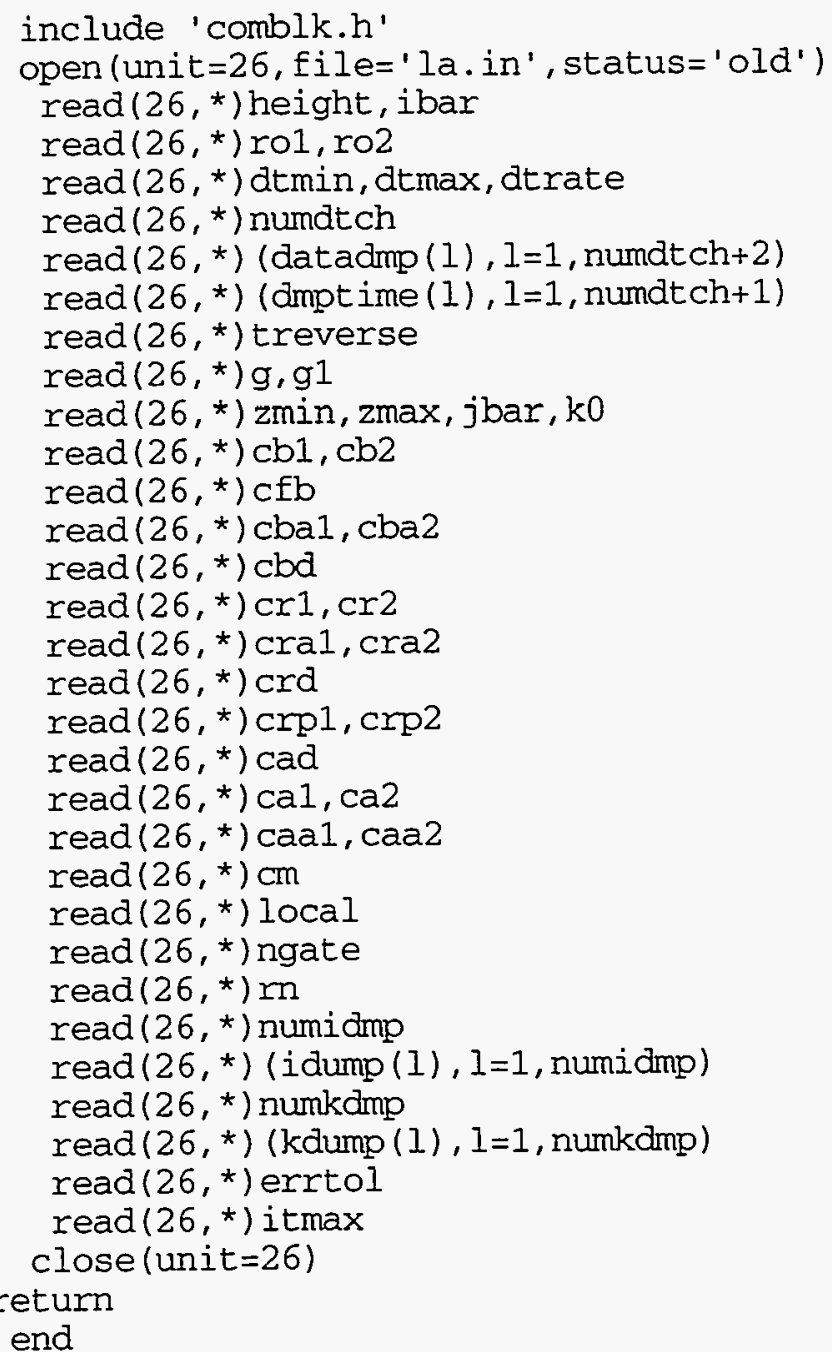

c234567890123456789012345678901234567890123456789012345678901234567890

subroutine setup

include 'param.h'

include 'comblk.h'

intface $=$ height $/ 2.0$

$I 1=i b a r / 2$

$d z=($ max - zmin $) / f l o a t(j b a r)$

$d y=$ height $/$ float (ibar)

atwood $=($ ro2 - rol $) /($ ro2 + ro1 $)$

denratio $=$ ro2/rol

runtime $=$ datadmp (numdtch +2$)$

$d t=d t \max$

$y(0)=0.0$

$y c(0)=-(d y+$ height $) / 2.0$

iitop $=$ ibar -4

iibottom $=4$

do $10 i=1$, ibar +1

if (i.lt.I1) then

$$
M(i)=r o 1 * d y
$$

alphal $(i)=1.0$

alpha2 $(i)=0.0$

$\operatorname{mass} 1(i)=M(i)$

$\operatorname{mass} 2(i)=0.0$ 


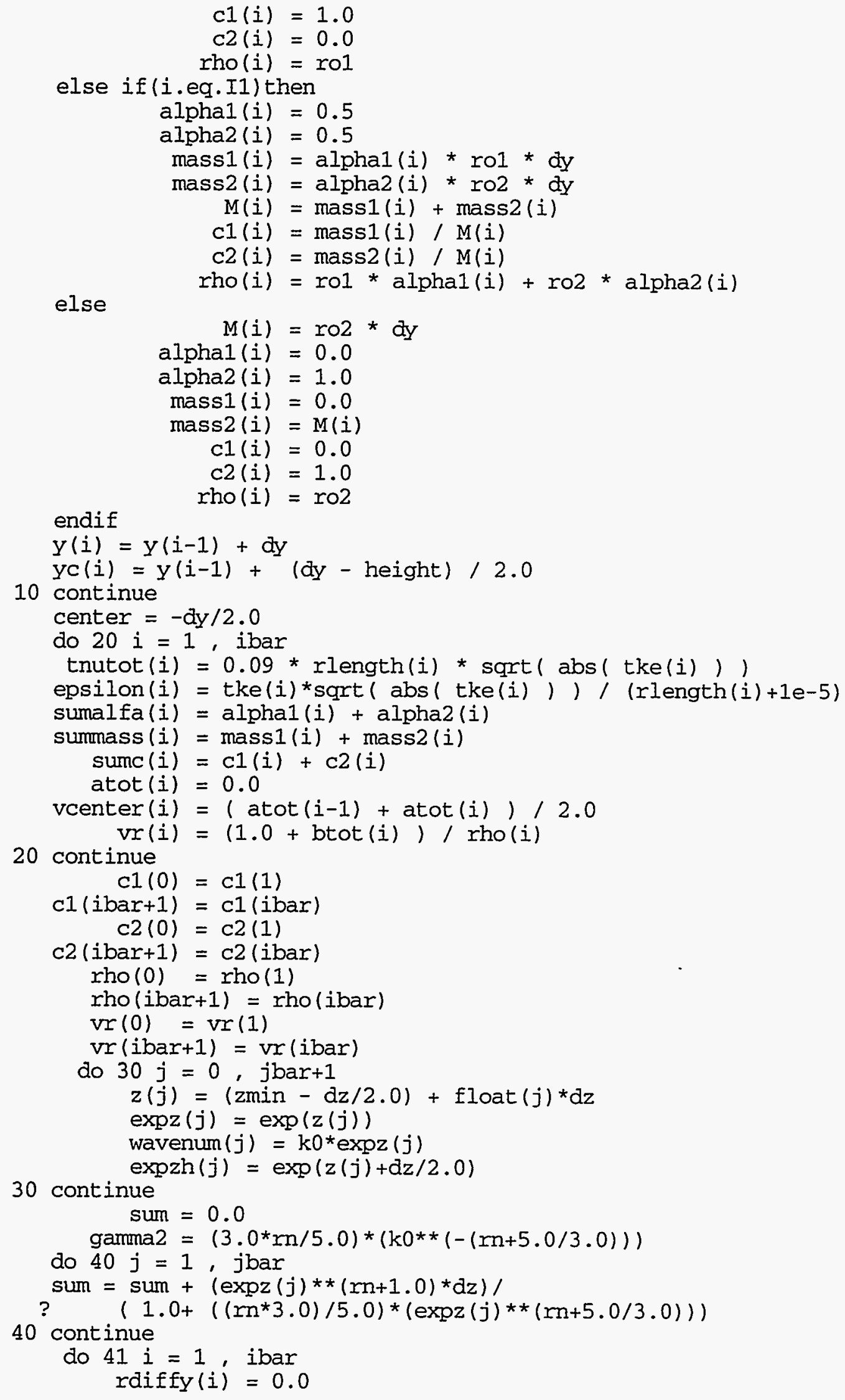




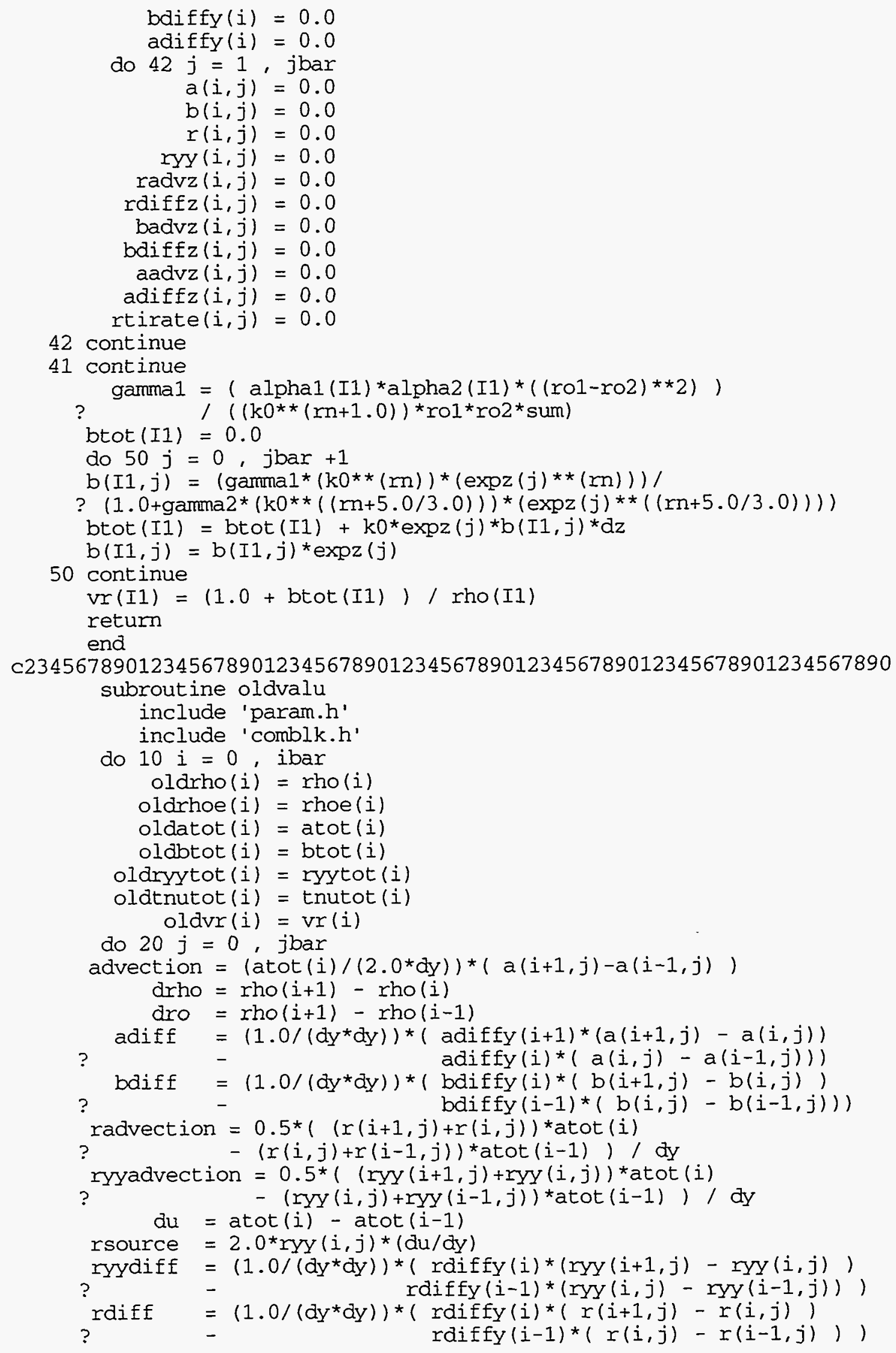




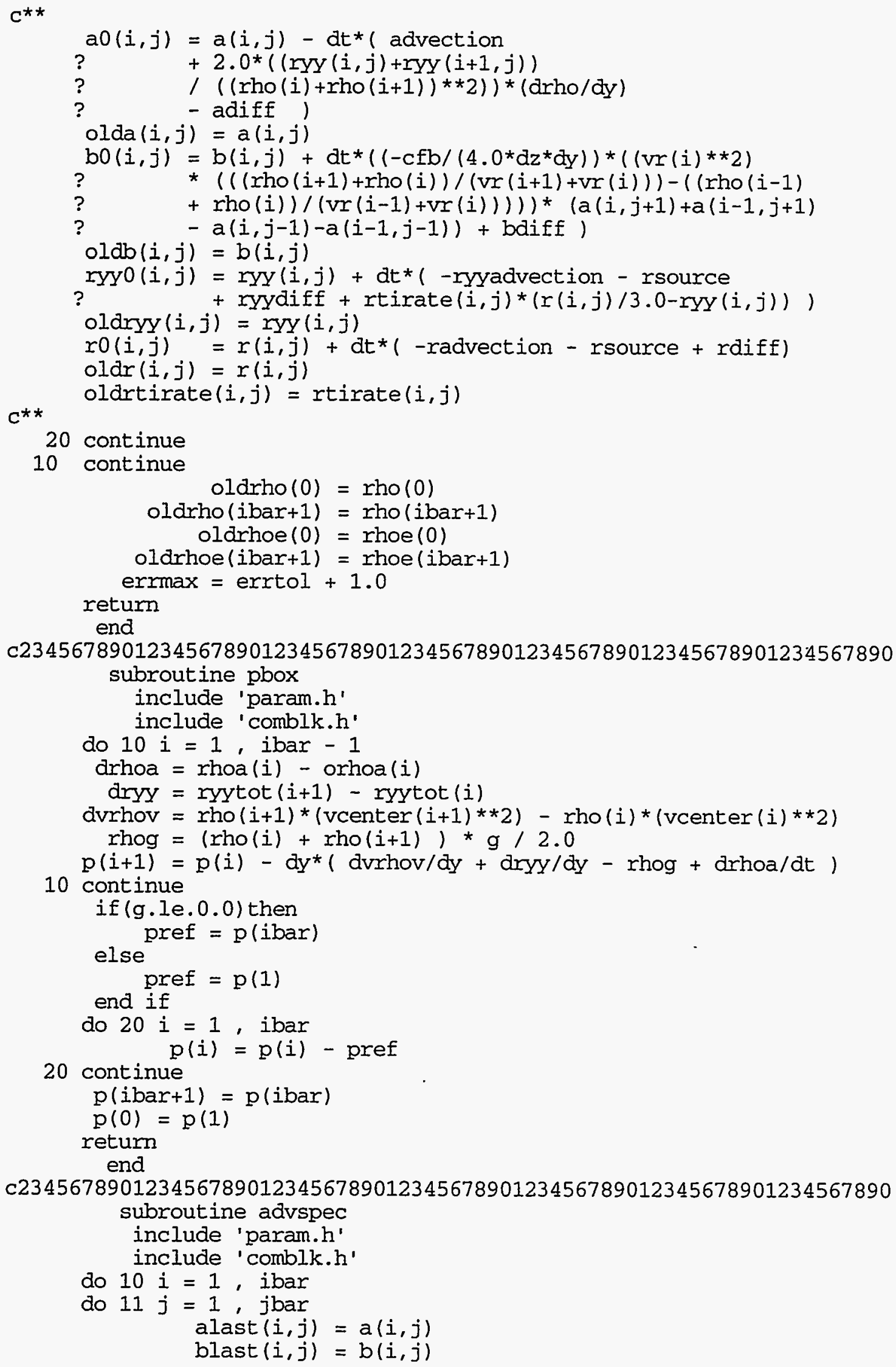




$$
\begin{aligned}
\operatorname{ry} \text { last }(i, j) & =\operatorname{ryY}(i, j) \\
\operatorname{rlast}(i, j) & =r(i, j)
\end{aligned}
$$

11 continue

10 continue

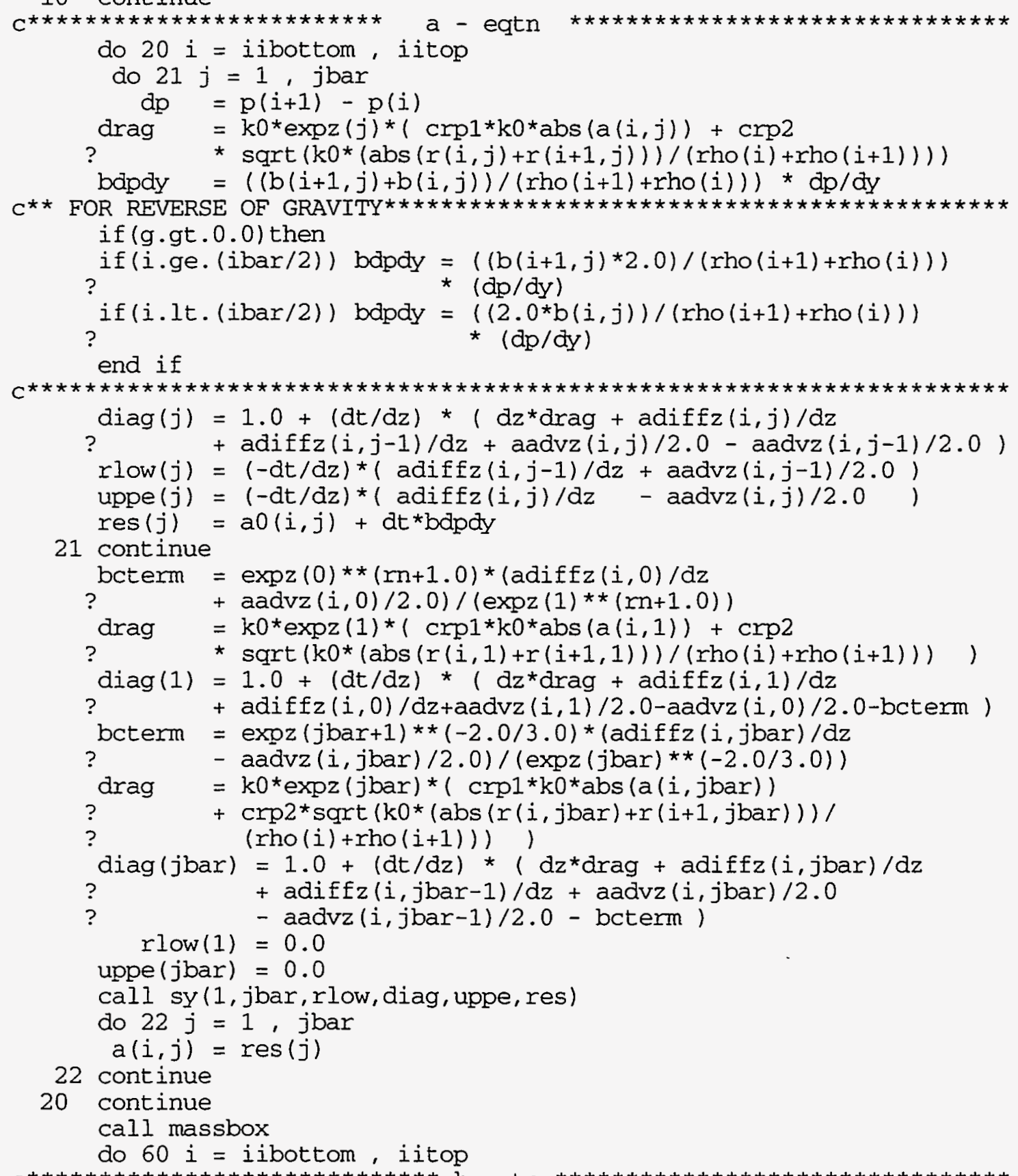

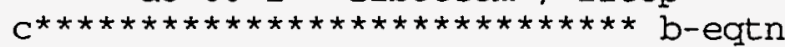

do $30 \mathrm{j}=1$, jbar

$\operatorname{diag}(j)=1.0+(d t / d z) *(\operatorname{bdiff} z(i, j) / d z+\operatorname{badvz}(i, j) / 2.0$

?

$+\operatorname{bdiffz}(i, j-1) / \mathrm{dz}-\operatorname{badvz}(i, j-1) / 2.0)$

$\operatorname{rlow}(j)=(-d t / d z) *(\operatorname{bdiff} z(i, j-1) / d z+\operatorname{badvz}(i, j-1) / 2.0)$

uppe $(j)=(-d t / d z) *(\operatorname{bdiffz}(i, j) / d z-\operatorname{badv} z(i, j) / 2.0)$

bdriver $=\left((2.0 *\right.$ rho(i) $\left.-r o 1-r o 2) /\left(\operatorname{rol}{ }^{*} r o 2\right)\right)$

? $\quad *((a) i, j) *($ rho $(i)+\operatorname{rho}(i+1))$

? $\quad-a(i-1, j) *(r h o(i-1)+r h o(i))) /(2.0 * d y))$

$\operatorname{res}(j)=\mathrm{b} 0(i, j)+d t *$ bdriver 
30 continue

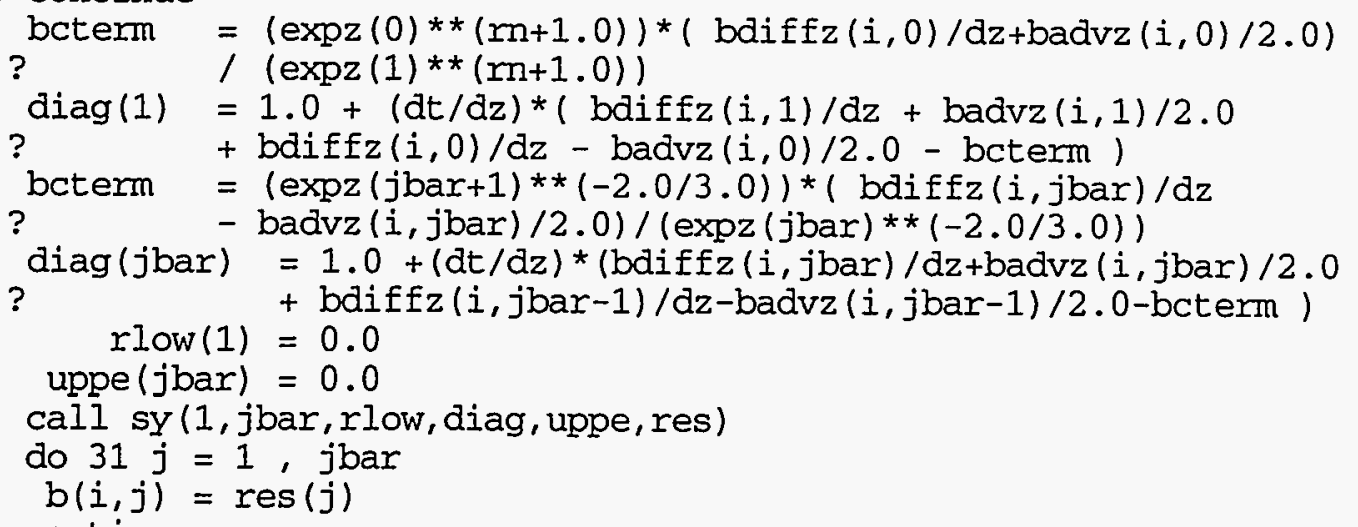

31 continue

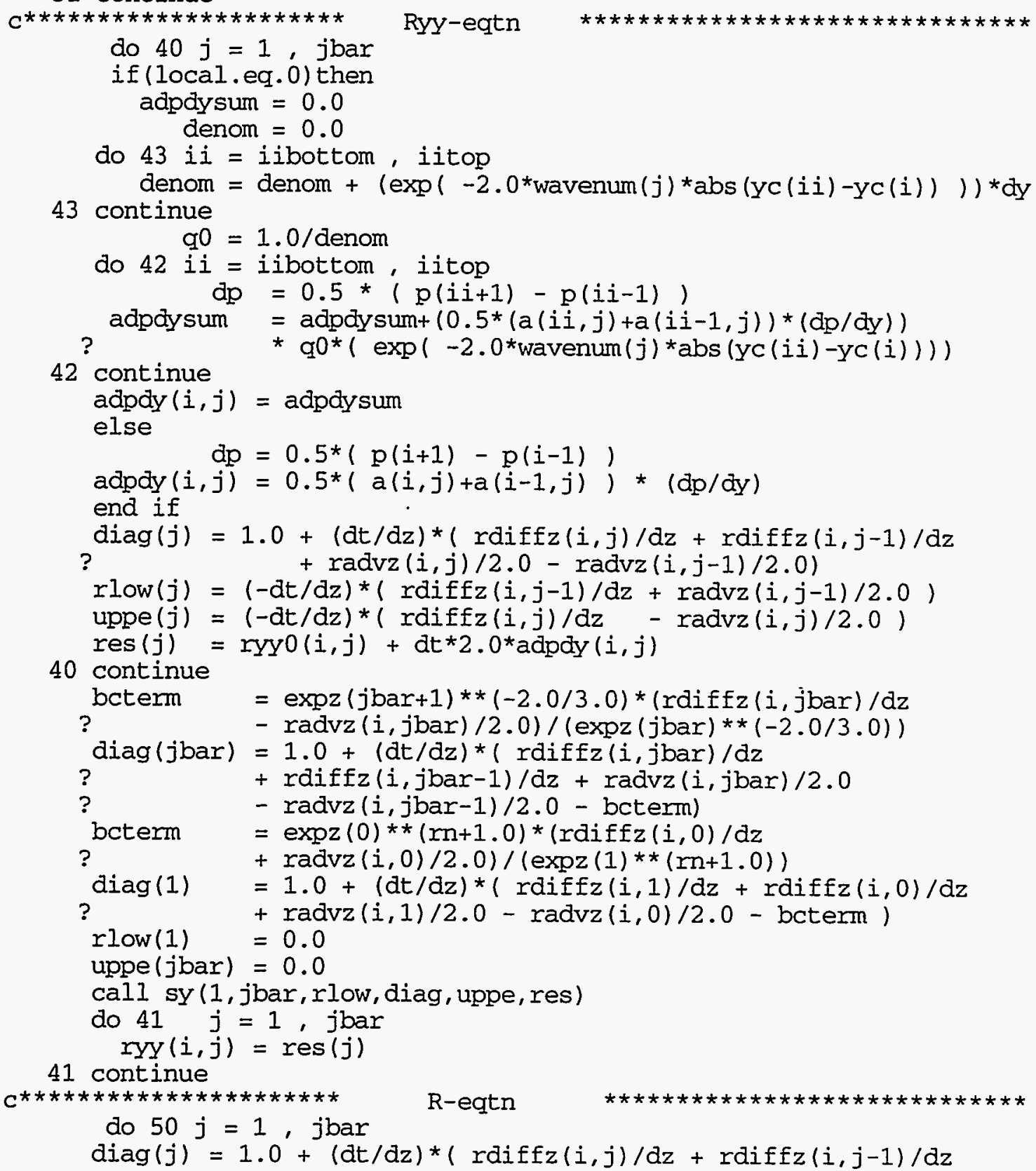


$+\operatorname{radvz}(i, j) / 2.0-\operatorname{radvz}(i, j-1) / 2.0)$

$\operatorname{rlow}(j)=(-d t / d z) *(\operatorname{rdiffz}(i, j-1) / d z+\operatorname{radvz}(i, j-1) / 2.0)$

uppe $(j)=(-d t / d z) *(\operatorname{rdiffz}(i, j) / d z-\operatorname{radvz}(i, j) / 2.0)$

$\operatorname{res}(j)=r 0(i, j)+d t * 2.0 * \operatorname{adpdy}(i, j)$

50 continue

bcterm $=\operatorname{expz}(j$ bar +1$) * *(-2.0 / 3.0) *(r \operatorname{diffz}(i, j$ bar $) / \mathrm{dz}$

? $\quad-\operatorname{radvz}(i, j$ bar $) / 2.0) /(\operatorname{expz}(j \operatorname{bar}) * *(-2.0 / 3.0))$

$\operatorname{diag}(j$ bar $)=1.0+(\mathrm{dt} / \mathrm{dz}) *(\operatorname{rdiffz}(i, j$ bar $) / \mathrm{dz}$

? $\quad+\operatorname{rdiffz}(i, j \operatorname{bar}-1) / d z+\operatorname{radvz}(i, j b a r) / 2.0$

? $\quad-\operatorname{radvz}(i, j$ bar -1$) / 2.0-$ bcterm)

bcterm $=\exp z(0) * *(r n+1.0) *(\operatorname{rdiffz}(i, 0) / \mathrm{dz}$

?

$\operatorname{diag}(1)=1.0+(\mathrm{dt} / \mathrm{dz}) *(\operatorname{rdiffz}(i, 1) / \mathrm{dz}+\operatorname{rdiffz}(i, 0) / \mathrm{dz}$

?

rlow (1)

$+\operatorname{radvz}(i, 1) / 2.0-\operatorname{radvz}(i, 0) / 2.0-$ bcterm $)$

uppe $(j$ bar $)=0.0$

call sy $(1$, jbar, rlow, diag, uppe, res)

do $51 j=1$, jbar

$r(i, j)=\operatorname{res}(j)$

$\mathrm{b} 22(i, j)=\operatorname{ryy}(i, j) / r(i, j)-1.0 / 3.0$

51 continue

60 continue call scales

C.

$$
\begin{aligned}
& \text { do } 70 i=0 \text {, ibar }+1 \\
& r(i, j \text { bar }+1)=r(i, j \text { bar }) *(\exp z(j b a r+1) * *(-2.0 / 3.0)) / \\
& \text { ? } \\
& (\operatorname{expz}(j \text { bar }) * *(-2.0 / 3.0)) \\
& \begin{aligned}
r y y(i, j b a r+1)= & \operatorname{ryy}(i, j \text { jar }) *(\exp z(j \text { bar } 1) * *(-2,0 / 3,0) \\
& (\exp z(j \text { bar }) * *(-2.0 / 3.0)) \\
b(i, j b a r+1)= & b(i, j \text { bar }) *(\exp z(j \operatorname{bar}+1) * *(-2.0 / 3.0)) /
\end{aligned} \\
& \text { ? } \quad(\operatorname{expz}(j \mathrm{bar}) * \star(-2.0 / 3.0)) \\
& a(i, j \text { bar }+1)=a(i, j \text { bar }) *(\operatorname{expz}(j \operatorname{bar}+1) * *(-2.0 / 3.0)) / \\
& \text { ? } \quad(\operatorname{expz}(j \text { bar }) * *(-2.0 / 3.0)) \\
& r(i, 0)=r(i, 1) *(\operatorname{expz}(0) * *(r n+1.0)) / \\
& \text { ? } \quad \operatorname{ryy}(i, 0)=\operatorname{ryy}(i, 1) *(\operatorname{expz}(0) * *(m+1.0)) / \\
& \text { ? } \quad(\operatorname{expz}(1) * *(r n+1.0)) \\
& b(i, 0)=b(i, 1) *(\exp z(0) * *(m+1.0)) / \\
& (\exp z(1) * *(r n+1.0)) \\
& a(i, 0)=a(i, 1) *(\exp z(0) * *(r n+1.0)) / \\
& \text { ? } \quad(\operatorname{expz}(1) * \star(r n+1.0))
\end{aligned}
$$

71 continue

$$
\begin{aligned}
& \text { do } 72 \quad j=0 \text {, jbar } \\
& \text { turbvel }=\operatorname{sqrt}(\operatorname{abs}(k 0 *(r(i, j)+r(i, j+1)) /(2.0 * r h o(i)))) \\
& \text { avelocity }=(0.25) *(\operatorname{abs}(a(i, j))+\operatorname{abs}(a(i, j+1))+\operatorname{abs}(a(i-1, j)) \\
& \text { ? } \quad+\operatorname{abs}(a(i-1, j+1))) \\
& \text { bdiffz }(i, j)=k 0 * \operatorname{expzh}(j) *(\mathrm{cb} 2 * \text { turbvel }+\mathrm{cba} 2 * k 0 * \text { avelocity }) \\
& \operatorname{badvz}(i, j)=k 0 * \exp z h(j) *((\mathrm{cb} 1+\mathrm{cb} 2) * \operatorname{turbve} 1+(\mathrm{cba} 1+\mathrm{cba} 2) \\
& \text { ? } \\
& \text { * } \mathrm{kO} \text { *avelocity) }
\end{aligned}
$$




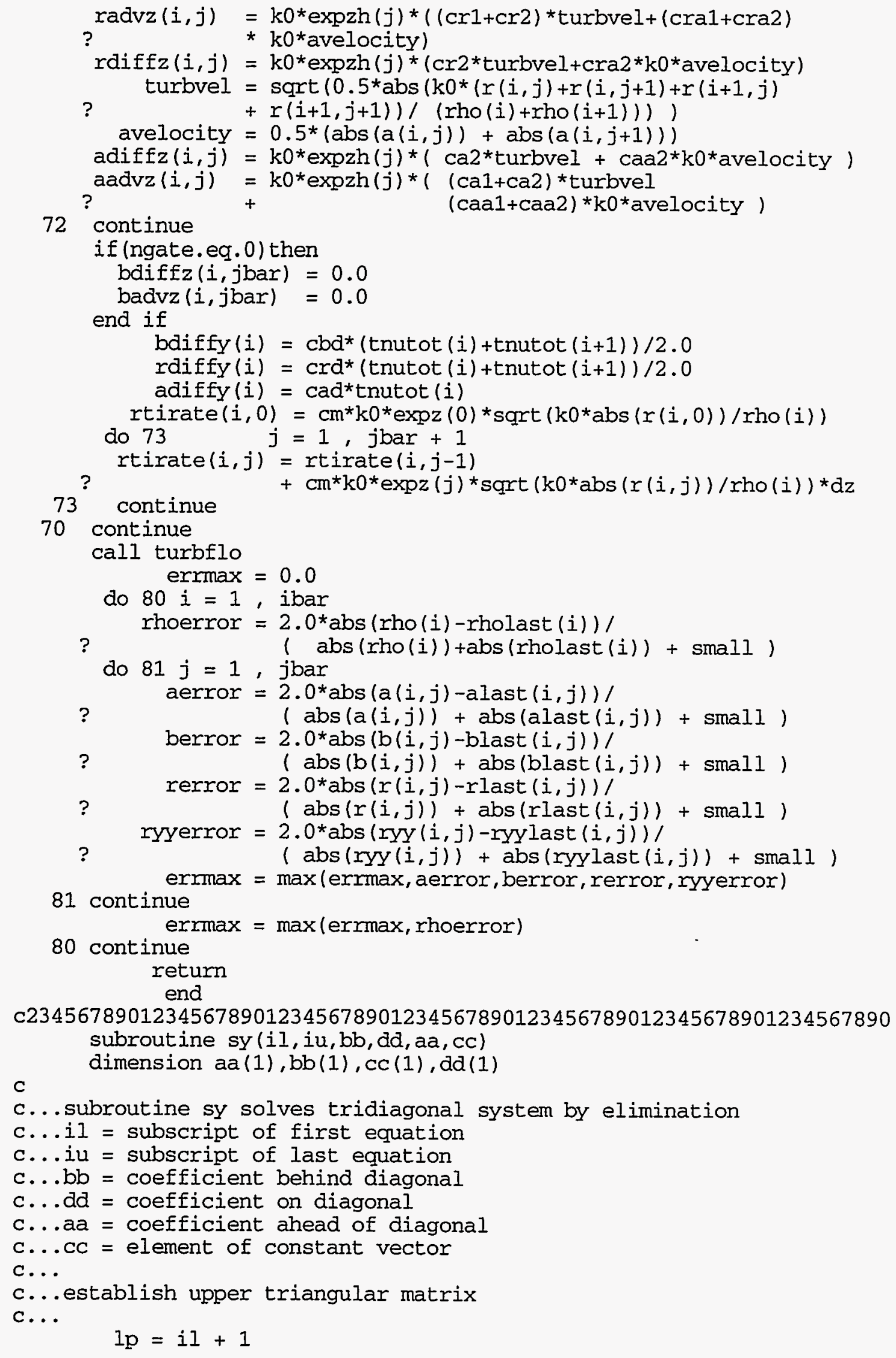




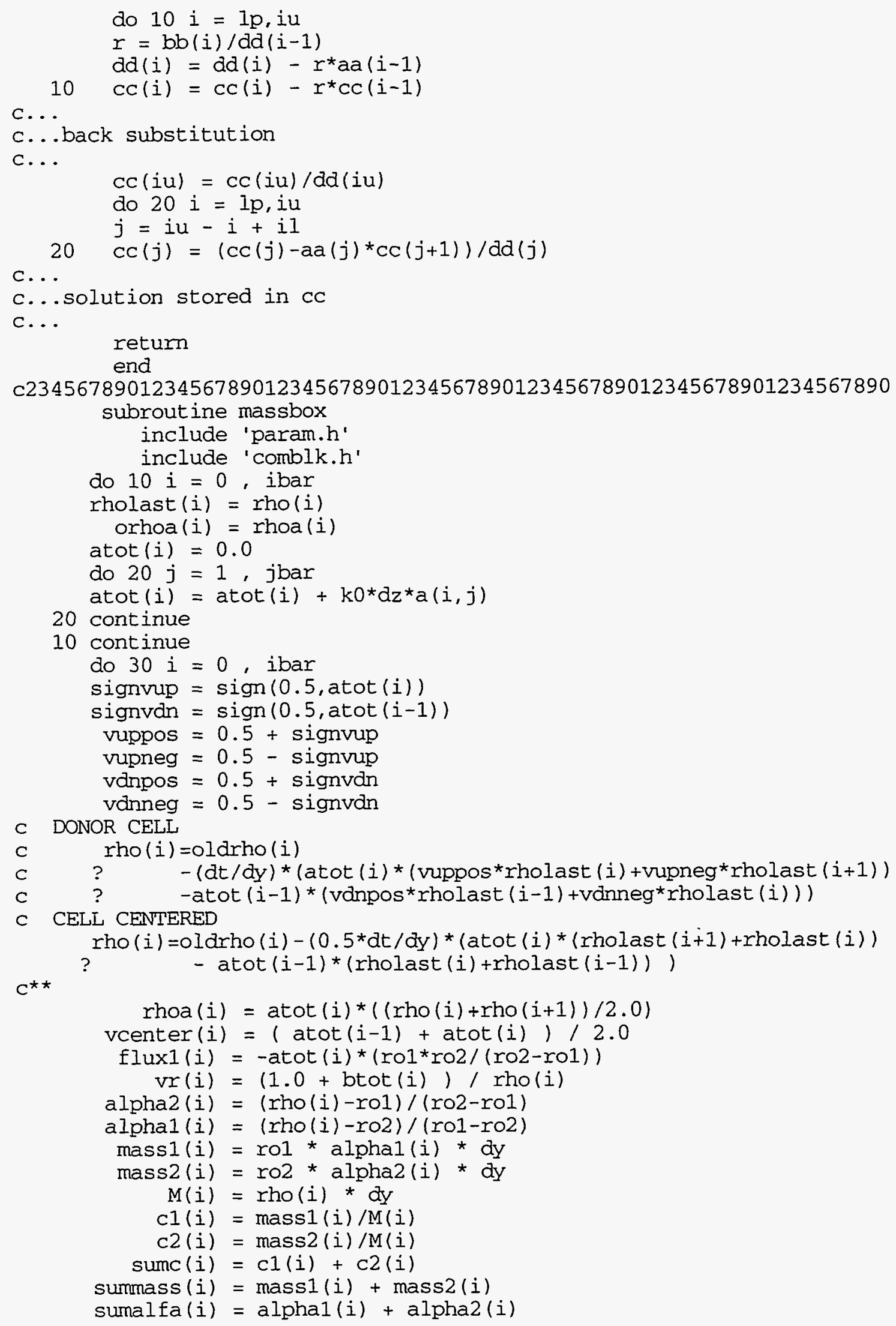


if ( (sumc (i).1t.0.99) or. (sumc (i).gt.1.01)) then

quit $=1$

call print

call grapher

call maxvalu

write $(20, *)$ 'CRASHED IN MASSBOX'

end if

30 continue

$\mathrm{k}=0$

do $40 i=1$, ibar

if $(k$. eq. 0$)$ then

if ( (alpha1 (i).1t.1.0) .or. (alphal (i).gt.1.0) .or.

? (alpha2(i).It.0.0).or. (alpha2(i).gt.0.0)) then

$\mathrm{k}=1$

iibottom $=i-10$

end if

end if

40

continue

do $50 i=i b a r, 1,-1$

if (k.eq.1) then

if ( (alpha2 (i).1t.1.0) .or. (alpha2 (i) .gt.1.0) .or.

? (alphal(i).1t.0.0).or. (alphal(i).gt.0.0)) then

$\mathrm{k}=0$

iitop $=i+10$

end if

end if

50 continue

$\operatorname{atot}(0)=\operatorname{atot}(1)$

atot $($ ibar $)=\operatorname{atot}($ ibar -1$)$

rhoa $(0)=$ rhoa $(1)$

rhoa (ibar) $=$ rhoa (ibar -1$)$

rho $(0)=$ rho (1)

rho $($ ibar +1$)=$ rho (ibar)

$$
\operatorname{vr}(0)=\operatorname{vr}(1)
$$

$\operatorname{vr}($ ibar +1$)=\operatorname{vr}($ ibar $)$

$c 1(0)=c 1(1)$

$\mathrm{c} 2(0)=\mathrm{c} 2(1)$

$c 1($ ibar +1$)=c 1$ (ibar)

$c 2($ ibar +1$)=c 2$ (ibar)

return

end

c234567890123456789012345678901234567890123456789012345678901234567890

subroutine turbflo include 'param.h'

include 'comblk.h'

do $10 i=1$, ibar

btotlast $(i)=$ btot $(i)$

btot $(i)=0.0$

$\operatorname{tke}(i)=0.0$

tnutot $(i)=0.0$

ryytot $(i)=0.0$

$\operatorname{rtot}(i)=0.0$

configb $(i)=\operatorname{alpha1}(i) * a l p h a 2(i) *((r o 1-r o 2) * * 2) /($ rol*ro2)

do $20 j=1$, jbar

$\operatorname{btot}(i)=b \operatorname{tot}(i)+k 0 * b(i, j) * d z$

tke $(i)=$ tke $(i)+k 0 * r(i, j) * \mathrm{dz} /(2.0 * r h o(i))$

tnutot $(i)=\operatorname{tnutot}(i)+k 0 * d z^{\star} \operatorname{sqrt}(\operatorname{abs}(r(i, j) / r h o(i))) / \operatorname{expz}(j)$

ryytot $(i)=$ ryytot $(i)+k 0 *$ ryy $(i, j) * d z$ 


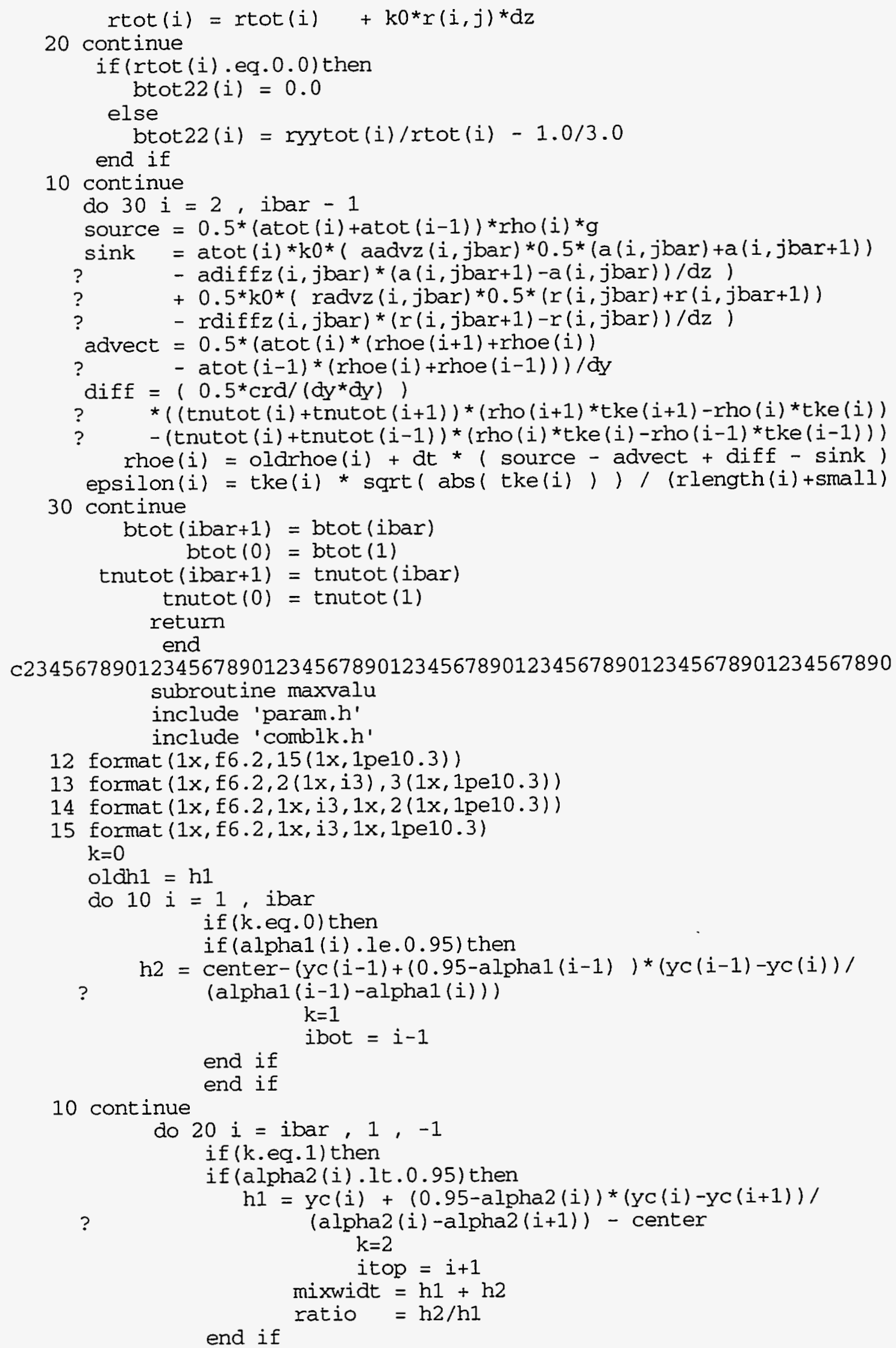


end if

continue

$\operatorname{smax}=0.0$

tkemax $=0.0$

tnumax $=0.0$

bmax $=0.0$

epsimax $=0.0$

atotmax $=0.0$

almax $=0.0$

blmax $=0.0$

ryylmax $=0.0$

do $30 i=i$ bot , itop

smax $=\max (r l$ length $(i), \operatorname{smax})$

almax $=\max ($ alength $(i)$, almax $)$

blmax $=\max (b l e n g t h(i), b l$ max $)$

ryylmax $=\max$ (ryylength $(i)$, ryylmax)

tkemax $=\max ($ tke $(i)$, tkemax)

tnumax $=\max ($ tnutot $(i)$, tnumax $)$

bmax $=\max (b t o t(i), b \max )$

epsimax $=\max ($ epsilon $(i)$, epsimax)

atotmax $=\max (\operatorname{abs}($ atot $(i))$, abs (atotmax) $)$

30 continue

atotmax $=$ atotmax ${ }^{*} \operatorname{sign}(1.0, \operatorname{atot}(i))$

$$
\begin{aligned}
\text { oldcapx } & =\text { capx } \\
\text { capx } & =\text { atwood*abs }(g) *(\text { time**2) }
\end{aligned}
$$

denom $=$ capx-oldcap $\mathrm{x}$

if (denom.eq. 0.0) then

alfa $=0.0$

else

end if

write $(28,12)$ time, smax, tkemax, tnumax, bmax, epsimax, atotmax,

$\$$

do $40 i=1$, ibar

write $(34,14)$ time, $i, y c(i)$, btot 22 (i)

40 continue

do $50 i=1$, numidmp

ipcell = idump (i)

write $(35,15)$ time, ipcell, btot 22 (ipcell)

do $60 \mathrm{j}=1$, jbar

write $(25,13)$ time, $j$, ipcell, $z(j)$, wavenum $(j), b 22$ (ipcell, $j$ )

60 continue

50 continue

return

end

c234567890123456789012345678901234567890123456789012345678901234567890

subroutine scales

include 'param.h'

include 'comblk.h'

do $10 i=1$, ibar

$c * \star \star \star \star \star \star \star \star \star \star \star \star \star \star \star \star \star * *$ a-length scale

$\operatorname{amax}=0.0$

$\mathrm{y} 1=0.0$

$\mathrm{y} 2=0.0$

$\mathrm{y} 3=0.0$

$\mathrm{x} 1=0.0$

$\mathrm{x} 2=0.0$

$\mathrm{x} 3=0.0$ 
do $20 j=1, j$ bar

if (abs $(a(i, j) / \exp z(j))$.gt .amax) then

$\operatorname{amax}=\operatorname{abs}(a(i, j) / \operatorname{expz}(j))$

$y_{1}=\operatorname{abs}(a(i, j-1) / \exp z(j-1))$

$y 2=\operatorname{abs}(a(i, j) / \operatorname{expz}(j))$

$y 3=\operatorname{abs}(a(i, j+1) / \exp z(j+1))$

$\mathrm{x} 1=$ wavenum $(j-1)$

$x 2=\operatorname{wavenum}(j)$

end if

$x 3=$ wavenum $(j+1)$

20 continue

if (amax.eq. 0.0) then

alength $(i)=0.0$

else

alength $(i)=1.0 / \mathrm{fx}(\mathrm{x} 1, \mathrm{x} 2, \mathrm{x} 3, \mathrm{y} 1, \mathrm{y} 2, \mathrm{y} 3)$ end if

$C^{\star \star \star \star \star * \star \star \star \star \star * ~}$

bmax $=0.0$

$y 1=0.0$

$\mathrm{Y}^{2}=0.0$

$y^{3}=0.0$

$\mathrm{x} 1=0.0$

$\mathrm{x} 2=0.0$

$x 3=0.0$

do $30 j=1$, jbar

if $((b(i, j) / \exp z(j))$.gt.bmax $)$ then

$b \max =b(i, j) / \exp z(j)$

$y 1=b(i, j-1) / \operatorname{expz}(j-1)$

$y 2=b(i, j) / \exp z(j)$

$y^{3}=b(i, j+1) / \exp z(j+1)$

$\mathrm{x} 1=$ wavenum $(j-1)$

$x 2=\operatorname{wavenum}(j)$

$x 3=\operatorname{wavenum}(j+1)$

end if

30

continue

if (bmax.eq. 0.0) then

blength $(i)=0.0$

else

blength $(i)=1.0 / f x(x 1, x 2, x 3, y 1, y 2, y 3)$

end if

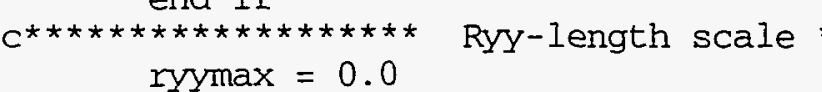

$\mathrm{y} 1=0.0$

$\mathrm{y} 2=0.0$

$\mathrm{y} 3=0.0$

$\mathrm{x} 1=0.0$

$\mathrm{x} 2=0.0$

$x^{3}=0.0$

do $40 j=1$, jbar

if ( (ryy $(i, j) / \operatorname{expz}(j))$.gt.ryymax) then

ryymax $=\operatorname{ryy}(i, j) / \operatorname{expz}(j)$

$y 1=\operatorname{ryy}(i, j-1) / \exp z(j-1)$

$y 2=\operatorname{ryy}(i, j) / \exp z(j)$

$y 3=\operatorname{ryy}(i, j+1) / \exp z(j+1)$

$x 1=\operatorname{wavenum}(j-1)$

$x 2=\operatorname{wavenum}(j)$

end if

$x 3=\operatorname{wavenum}(j+1)$ 


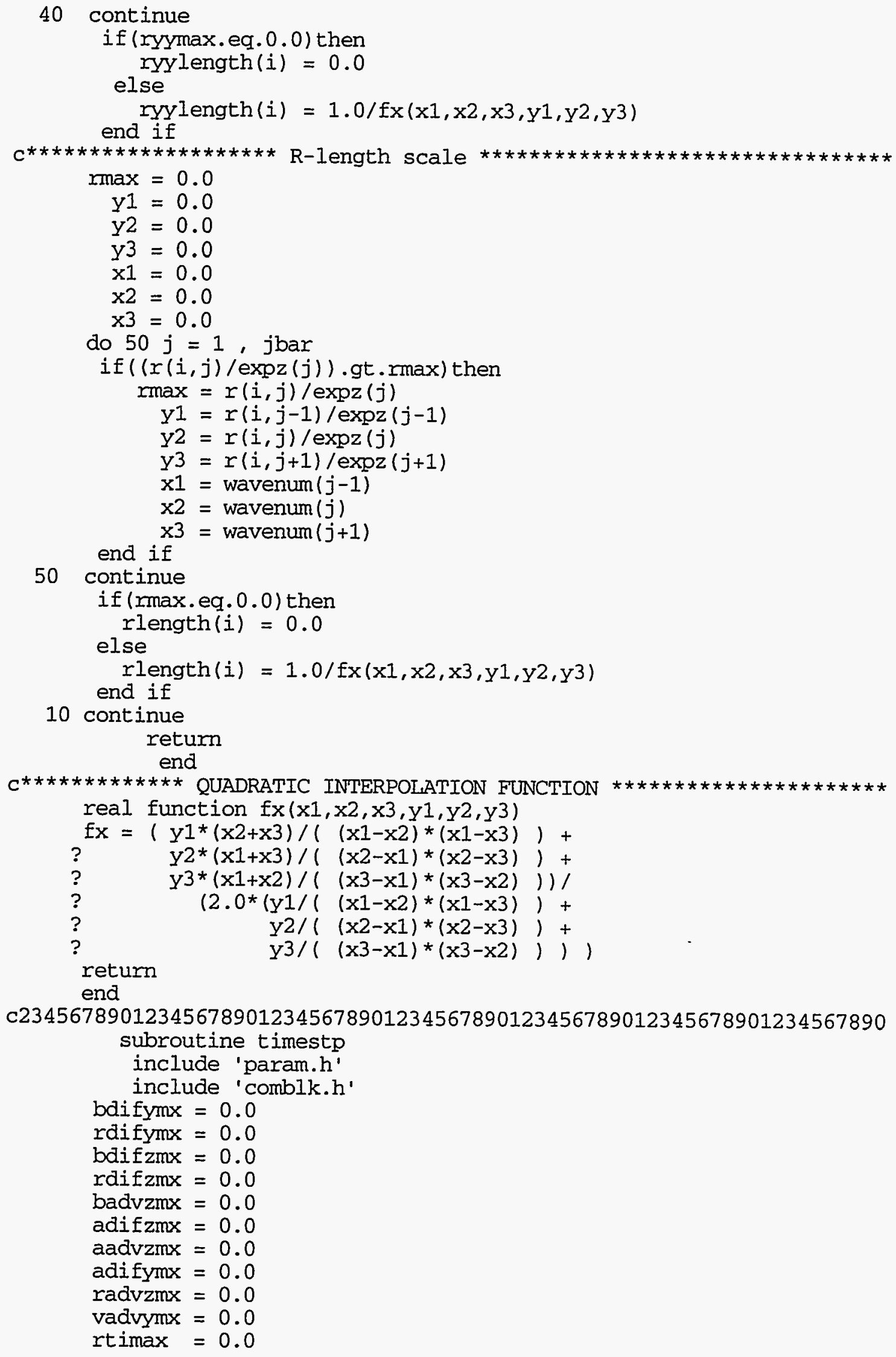




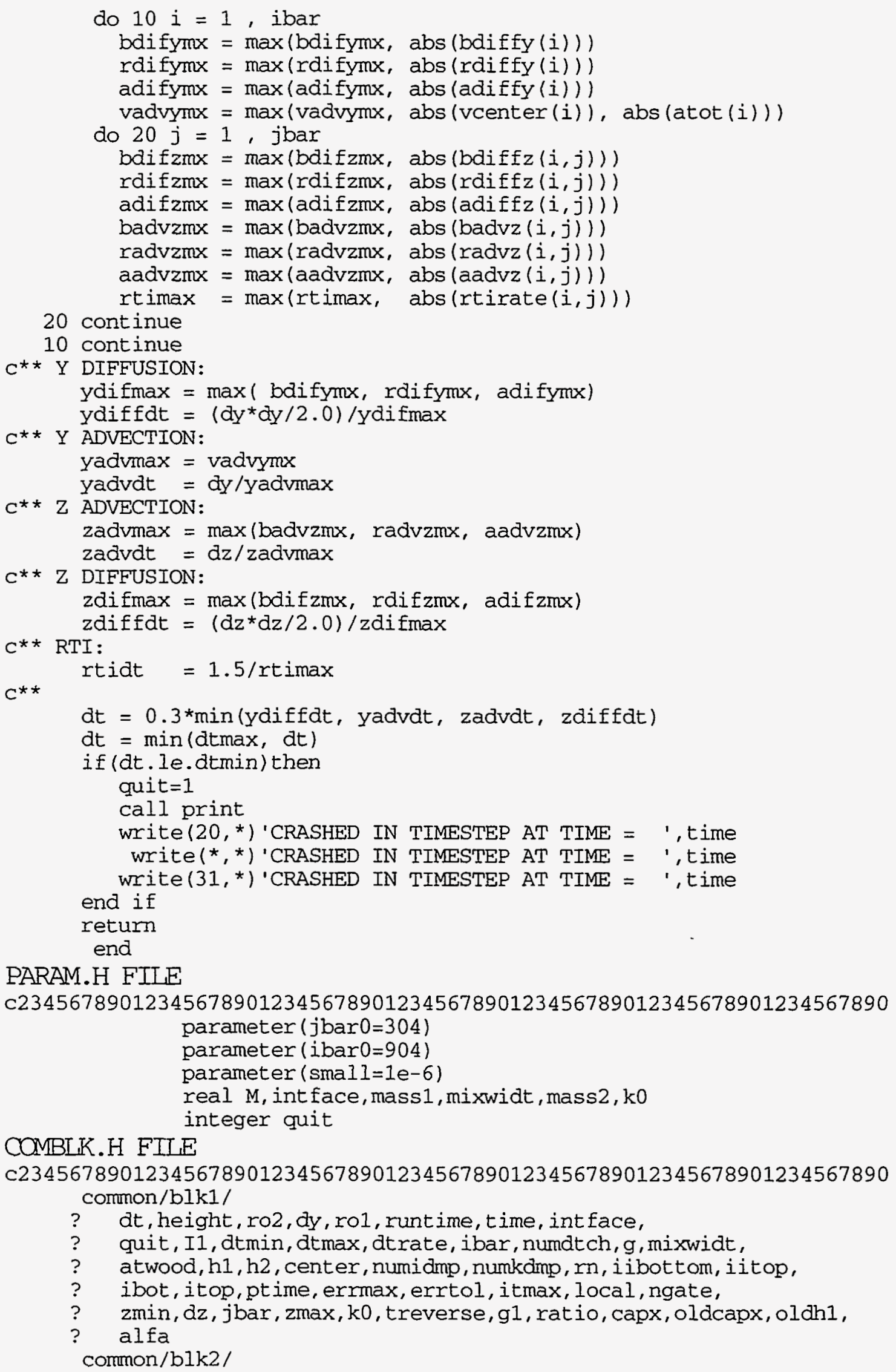




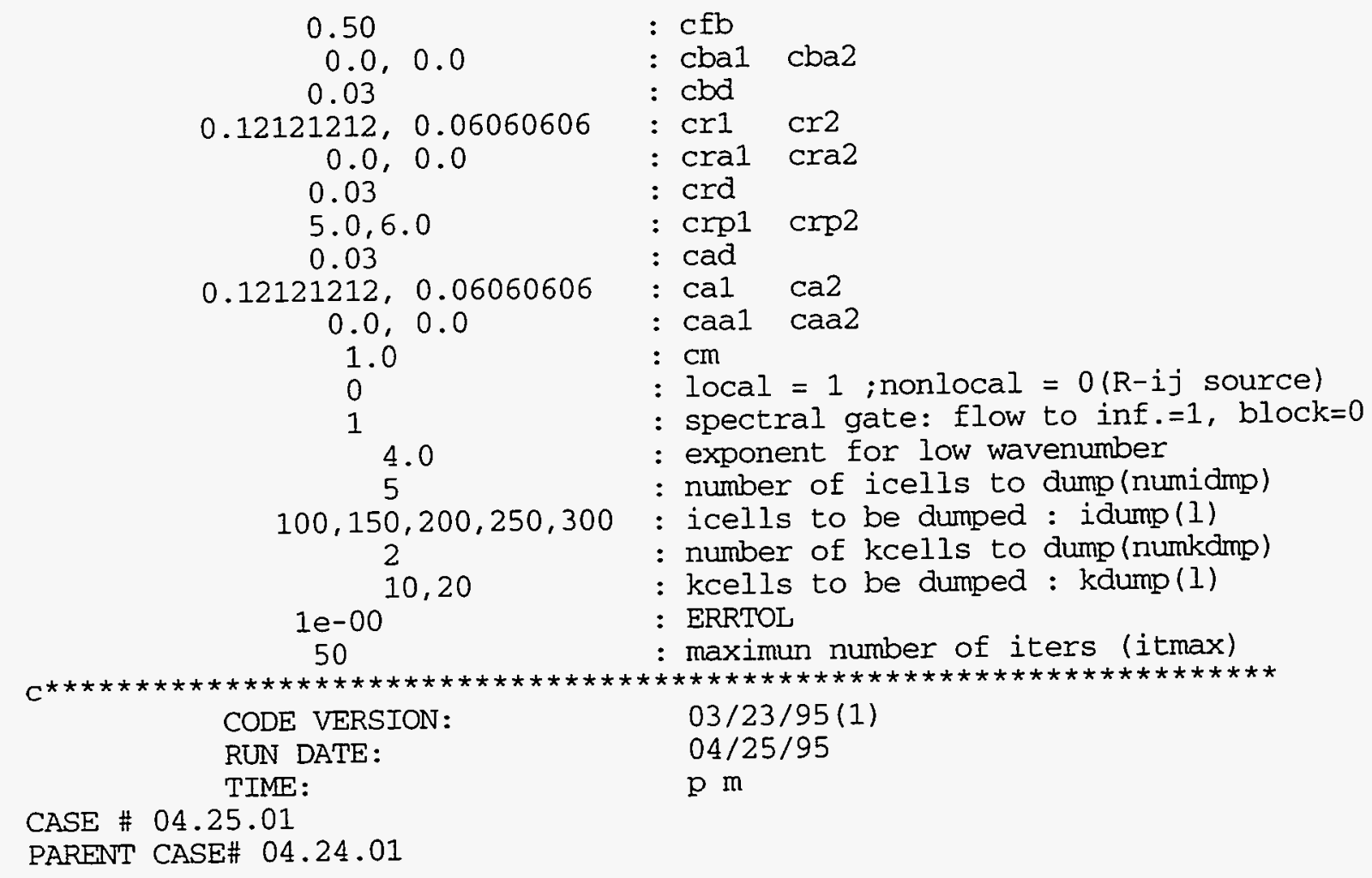




\section{Bibliography}

ALLRED, J.C, \& BLOUNT, G.H. 1953 Experimental studies of Taylor instability. Los Alamos National Laboratory, Los Alamos National Laboratory Report, LA-1600.

ANDERSON, D. A., TANNEHILL, J. C. \& PLETCHER, R. H. 1984 Computational Fluid Mechanics and Heat Transfer. Hemisphere Publishing Corporation. pp. 221-235.

ANUCHINA, N. N., KUCHERENKO, YU. A., NEUVAZHAEV, V. E., OGIBINA, V. N., SHIBARSHOV, L. I. \& YAKOVLEV, V. G. 1978 Turbulent mixing at the accelerating interface between fluids. Mechanics of Fluids, N 6, 157.

ANDREWS, M. J. 1986 Turbulent mixing by Rayleigh-Taylor instability. CFDU Report No. CFDU/86/10 (Ph. D. Dissertation)

ANDREWS, M. J. 1986 A "two-fluid" model of de-mixing in the saline experiment. CFDU Report No. CFDU/86/11

ANDREWS, M.J. \& SPALDING, D.B. 1990 A simple experiment to investigate twodimensional mixing by Rayleigh-Taylor instability. Phys. Fluids A., 922-927.

ANDREWS, M.J. 1992 An experimental study of turbulent mixing by Rayleigh-Taylor instabilities and a two-fluid model of the mixing phenomena. Advances in Compressible Turbulent Mixing (ed. Dannevik, W. P. Buckingham A. C. \& Leith, C. E.) National Technical Information Service, U.S. Department of Commerce, 7-19.

ANDRONOV, V.A., BAKHRAKH, S.M., MESHKOV,E.E., MOKHOV, V. V., NIKIFOROV, A. V., PEVNITSHII, A.V. \& TOLSHMYAKOV, A.I. 1976 Turbulent mixing at contact surface accelerated by shock waves. Sov. Phys. JETP 44(2), 424-427.

ANDRONOV, V.A., BAKHRAKH, S.M., MESHKOV,E.E., NIKIFOROV, V. V., PEVNITSHII, A.V. \& TOLSHMYAKOV, A.I. 1982 An experimental investigation and numerical modeling of turbulent mixing in one-dimensional flows. Sov. Phys.

Dokl. 27(5), 393-396.

ANISIMOV, V. I. \& POLYONOV, A. V. 1989 The statistical model of the turbulent mixing zone and its applying to the experiments analysis. International Workshop on Compressible Turbulent Mixing, Pleasanton, CA.

BATCHELOR, G. K. 1986 The theory of homogeneous turbulence. Cambridge University Press in the Cambridge Science Classics series 1982. Page 38.

BELENKII, S. Z. \& FRADKIN, E. S. 1965 The theory of turbulent mixing. Papers of Phys. Ins. of AS USSR, Vol. 29.

BELLMAN, R. \& PENNINGTON, R. H. 1954 Effects of surface tension and viscosity on Taylor instablity. Quarterly of Applied Mathematics 12, 151-162. 
BERTOGLIO, J. -P. 1982 A model of three-dimensional transfer in non-isotropic homogeneous turbulence. In Turbulent Shear Flows 3. (ed. L.J.S. Bradbury, F.

Durst, B.E. Launder, F.W. Schmidt \& J. H. Whitelaw). Springer-Verlag. 253-261.

BERTOGLIO, J.-P. \& JEANDEL, D. 1987 A simplified spectral closure for inhomogeneous turbulence: Application to the boundary layer. In Turbulent Shear Flows 5. (ed.

L.J.S. Bradbury, F. Durst, B.E. Launder, F.W. Schmidt \& J. H. Whitelaw). SpringerVerlag. $19-30$

BESNARD, D. C., HARLOW, F. H., RAUENZAHN, R. M. 1987 Conservation and transport properties of turbulence with large density variations. Los Alamos National Laboratory, Los Alamos National Laboratory Report, LA-10911-MS.

BESNARD, D. C., HARLOW, F. H., RAUENZAHN, R. M. \& ZEMACH, C. 1990 Spectral transport model for turbulence. Los Alamos National Laboratory, Los Alamos National Laboratory Report, LA-11821-MS.

BESNARD, D. C., HARLOW, F. H., RAUENZAHN, R. M. \& ZEMACH, C. 1992 Turbulence transport equations for variable-density turbulence and their relationship to two-field models. Los Alamos National Laboratory, Los Alamos National Laboratory Report, LA-12303-MS.

BESNARD, D. C., HARLOW, F. H., RAUENZAHN, R. M. \& ZEMACH, C. 1995 Spectral transport model for turbulence. Theoretical and Computational Fluid Dynamics 7 . (Accepted for publication)

BIRKHOFF, G. 1954 Taylor instability and laminar mixing. Los Alamos Scientific Laboratory, Los Alamos Scientific Laboratory Report LA-1862.

BIRKHOFF, G. 1956 Taylor Instability Appendices to report LA-1862. Los Alamos Scientific Laboratory, Los Alamos Scientific Laboratory Report, LA-1927.

BRADSHAW, P., CEBECI, T. \& WHITELAW, J. H. 1981 Engineering Calculation Methods for Turbulent Flows. Academic Press. Pages 38-49.

BROWN, G.L. \& ROSHKO, A. 1974 On density effects and large structure in turbulent mixing layers. J. Fluid Mech. 64(4), 775-816.

BURROWS, K.D., SMEETON, V.S. \& YOUNGS, D.L. 1984 Experimental Investigation of Turbulent Mixing by Rayleigh-Taylor Instability, II. AWRE Report 0 22/84.

CAMBON, C. 1979 Modélisation Spectrale en Turbulence Homogène Anisotrope. Thèse de Docteur-Ingénieru, Université Claude Bernard, Lyon.

CAMBON, C., JEANDEL, D. \& MATHIEU, J. 1981 Spectral modelling of homogeneous non-isotropic turbulence. J. Fluid Mech. 104, 247-264.

CASE, K. M. 1960 Taylor instability of an inverted atmosphere. Phys. Fluids 3, 366-368. 
CHANDRASEKHAR, S. 1954 Stochastic problems in physics and astronomy. In Selected Papers on Noise and Stochastic Processes (ed. Nelson Wax).Dover, New York. 1-89.

CHANDRASEKHAR, S. 1961 Hydrodynamic and Hydromagnetic Stability . Oxford University Press, Oxford, 428-514.

CHIEN, K.-Y. 1982 Predictions of channel and boundary-layer flows with a low-Reynoldsnumber turbulence model. AIAA Journal 20(1), 33-38.

CLARK, T.T. 1992 Spectral self-similarity of homogeneous anisotropic turbulence. Los Alamos National Laboratory, Los Alamos National Laboratory Report, LA-12284-T.

CLARK, T.T. \& SPITZ, P.B. 1995 Two-point correlation equations for variable density turbulence. Los Alamos National Laboratory, Los Alamos National Laboratory Report, LA-1267I-MS.

COLE, R.L. \& TANKIN, R.S. 1973 Experimental study of Taylor instability. Phys. Fluids 16(11), 1810-1815.

COOK, T.L., DEMUTH, R.B. \& HARLOW, F.H. 1981 PIC calculations of multiphase flow. J. Comp. Phys. 41(1), 51-67.

COOK, T.L. \& HARLOW, F.H. 1984a Virtual mass in multiphase flow. Int. J. Multiphase Flow 10(6), 691-696.

COOK, T.L. \& HARLOW, F.H. 1984b VORT: A computer code for bubbly two-phase flow. Los Alamos National Laboratory, Los Alamos National Laboratory Report, LA-1002I-MS.

COOK, T.L. \& HARLOW, F.H. 1986 Vortices in bubbly two-phase flow. Int. J. Multiphase Flow 12(1), 35-61.

CRANFILL, C. W. 1991 A multifluid turbulent-mix model. Los Alamos National Laboratory, Los Alamos National Laboratory Report, LA-UR-91-403.

CRANFILL, C. W. 1992 A new multifluid turbulent-mix model. Los Alamos National Laboratory, Los Alamos National Laboratory Report, LA-UR-92-2484.

DALY, B.J. 1967 Numerical study of two fluid Rayleigh-Taylor instability. Phys. Fluids 10(2), 297-307.

DALY, B. J. 1969 Numerical study of the effect of surface tension on interface instability. Phys. Fluids. 12(7), 1340-1354.

DALY, B.J. \& HARLOW, F.H. 1970 Transport equations in turbulence. Phys. Fluids 13(11), 2634-2649.

DALY, B.J. 1992 Turbulence in MESA-2D. Los Alamos National Laboratory, Los Alamos National Laboratory Report, LA-12257-MS. 
DAVIES, R.M. \& TAYLOR, G.I. 1949 The mechanics of large bubbles rising through extended liquids and through liquids in tubes. Proc. Roy. Soc. Lond. A 200, 375-390.

DEMUREN, A.O., LELE, S.K. \& DURBIN, P. 1994 Role of pressure diffusion in nonhomogeneous shear flows. Bulletin of the American Physical Society. Program for the 1994 Annual Meeting of the Division of Fluid Dynamics. 38 (12), Page 1954.

DREW, D.A. 1983 Mathematical modeling of two-phase flow. Ann. Rev. Fluid Mech. 15, 261-291.

DUFF, R.E., HARLOW, F.H. \& HIRT, C.W. 1962 Effects of diffusion on interface instability between gases. Phys. Fluids 5(4), 417-425.

EMMONS, H.W., CHANG, C.T. \& WATSON, B.C. 1960 Taylor instability of finite surface waves. J. Fluid Mech. 7, 177-193.

GARDNER, C. L., GLIMM, J., MCBRYAN, O., MENIKOFF, R., SHARP, D. H. \& ZHANG, Q. 1988 The dynamics of bubble growth for Rayleigh-Taylor unstable interface. Phys. Fluids A, 31, 447-465.

GLIMM, J., LI, X.L., ZHANG, Q., MENIKOFF, R. \& SHARP, D. 1992 Statistical Theories of Rayleigh-Taylor iinstability for compressible fluids. Advances in Compressible Turbulent Mixing (ed. Dannevik, W. P. Buckingham A. C. \& Leith, C. E.) National Technical Information Service, U.S. Department of Commece, 85-93.

GODEFERD, F. S. \& CAMBON, C. 1994 Detailed investigation of energy transfer in homogeneous stratified turbulence. Phys. Fluids 6(6), 2084-2100.

HANJALIC, K. \& LAUNDER, B. E. 1972 A Reynolds stress model of turbulence and its application to thin shear flows. J. Fluid Mech. 52(4), 609-638.

HANJALIC, K., LAUNDER, B.E. \& SCHIESTEL, R. 1980 Multiple-time scale concepts in turbulent transport modeling. In Turbulent Shear Flows-2 (ed. L. J. S. Bradbury, F. Durst, B. E. Launder, F. W. Schmidt \& J. H. Whitelaw). Springer-Verlag, 36-49.

HARLOW, F. H. \& WELCH, J. E. 1965 Numerical calculation of time-dependent viscous incompressible flow of fluid with free surface. Phys. Fluids 8, 2182-2189.

HARLOW, F.H. \& NAKAYAMA, P.I. 1967 Turbulence transort equations. Phys. Fluids 10(11), 2323-2331.

HARLOW, F. H. \& NAKAYAMA, P.I. 1968 Transport of turbulence energy decay rate. Los Alamos Scientific Laboratory Report LA-3854.

HARLOW, F. H. 1968 Transport of anisotropic or low-intensity turbulence. Los Alamos Scientific Laboratory Report LA-3947.

HARLOW, F. H. \& AMSDEN, A. A. 1971 Fluid dynamics. Los Alamos National Laboratory, Los Alamos Scientific Laboratory Report LA-4700. 
HARLOW, F.H. \& AMSDEN, A.A. 1975 Numerical calculation of multiphase fluid flow. J.Comp. Phys. 17, 19-52.

HEISENBERG, W. 1948 On the theory of statistical and isotropic turbulence. Proc. Roy. Soc. London A 195, 402-406.

HIDE, R. 1955 The character of the equilibrium of an incompressible heavy viscous fluid of variable density: An approximate theory. Proc. Cambridge Phil. Soc. 51, 179-201.

HINZE, J.O. 1987 Turbulence. Kingsport Press. Page 5, 23.

ILGEBUSI, J. O. \& SPALDING, D. B. 1985 An improved version of the K-W model of turbulence. J. Heat Transfer 107, 63-69.

JEANDEL, D., BRISON, J. F. \& MATHIEU, J. 1978 Modeling methods in physical and spectral space. Phys. Fluids 21(2), 169-182.

JONES, A.V. \& PROSPERETTI, A. 1985 On the suitability of first-order differential models for two-phase flow prediction. Int. J. Multiphase Flow, 11, 133-147.

JONES, W.P. 1980 Models for turbulent flows with variable density. VKI Lecture Series 1979-2, Prediction Methods for Turbulent Flows, Hemisphere Publ. Corp., New York.

JONES, W.P. \& WHITELAW, J.H. 1982 Calculation methods for reacting turbulent flows: A review. Combustion and Flame 48, 1-26.

JONES, W.P. \& LAUNDER, B.E. 1972 The prediction of laminarization with a twoequation model of turbulence. Int. J. Heat Mass Transfer, 15, 301-314.

JONES, W.P. \& LAUNDER, B.E. 1973 The calculation of low-Reynolds-number phenomena with a two-equation model of turbulence. Int. J. Heat Mass Transfer 16 1119-1130.

KOLMOGOROFF, A. N. 1941 Dissipation of energy in the locally isotropic turbulence. Compt. rend. acad. sci U.R.S.S., 32, 16-18.

KOVASZNAY, L. S. G. 1948 Spectrum of locally isotropic turbulence. J. Aeronautical Sciences. 745-753.

KRAICHNAN, R.H. 1958 Irreversible statistical mechanics of incompressible hydromagnetic turbulence. Phys. Rev. 109(5), 1407-1422.

KRAICHNAN, R.H. 1959 The structure of turbulence at very high Reynolds numbers. J. Fluid Mech. 5, 497-543.

KRAICHNAN, R.H. 1961 Dynamics of nonlinear stochastic systems. J. Math. Phys. 2(1), 124-148. 
KRAICHNAN, R.H. 1964 Decay of isotropic turbulence in the direct-interaction approximation. Phys. Fluids 7(7), 1030-1049.

KRAICHNAN, R.H. 1965 Lagrangian-history closure approximation for turbulence. Phys. Fluids 8(8), 575-598.

KRAICHNAN, R.H. 1966 Isotropic turbulence and inertial-range structure. Phys. Fluids 9(9), 1728-1752.

KRAICHNAN, R.H. 1971 An almost-Markovian Galilean-invariant turbulence model. J. Fluid Mech. 47(3), 512-524.

KRAICHNAN, R.H. 1972 Test-field model for inhomogeneous turbulence. J Fluid Mech. 56(2), 287-304.

KUCHERENKO, YU. A. \& SHIBARSHOV, L.I. 1986 Turbulent mixing at the plane interface between media with different densities and being subjected to the series of delta-shaped accelerations. Problems of Atomic Science \& Engineering (Series: Theor. \& Appl. Physics) 2, 3 .

KUCHERENKO, YU. A., TOMASHEV, G. G. \& SHIBARSHOV, L.I. 1988 Experimental study of gravitational mixing in the self-similar mode. Problems of Atomic Science \& Engineering (Series: Theoretical \& Applied Physics) 1, p. 13.

KUCHERENKO, YU. A., SHIBARSHOV, L.I., CHITAIKIN, V.I., BALABIN, S.I., \& PYLAEV, A.P. 1991 Experimental study of the gravitational turbulent mixing self-similar mode. 3rd International Workshop on The Physics Of Compressible Turbulent Mixing. Abbey of Royaumont (France), 427-454.

KUCHERENKO, YU. A., NEUVAZHAEV, V.E., \& PYLAEV, A.P. 1994 Behavior of a region of gravity-induced turbulent mixing under conditions leading to separation. Physics-Doklady (Official English Translation of Doklady Akademii Nauk). 39(2) 114-117.

KUO, K.K. 1986 Principles of Combustion. John Wiley \& Sons, New York, 444-446.

LAMB, H. 1931 Hydrodynamics. Dover Publications, Inc., New York, 6th edition. pp. $370,378,456,625$.

LAUNDER, B. E. 1990 Phenomenological modelling: Present ... and future? In Whither Turbulence? or Turbulence at the Crossroads (ed. J. L. Lumley). Sringer-Verlag. 439-485.

LAUNDER, B. E. \& SPALDING, D. B. 1972 Lectures in Mathematical Models of Turbulence. Academic Press (London).

LAUNDER, B. E. \& SPALDING, D. B. 1974 The numerical computation of turbulent flows. Comp. Methods in Applied Mech. and Engr. 3, 269-289. 
LAUNDER, B.E., REECE, G.J. \& RODI, W. 1975 Progress in the development of a Reynolds-stress turbulence closure. J. Fluid Mech. 68 (3), 537-566.

LEITH, C. E. 1967 Diffusion approximation to inertial energy transfer in isotropic turbulence. Phys. Fluids, 11, 671-673.

LEITH, C. E. 1971 Atmospheric predictability and two-dimensional turbulence. J. Atmos. Science 28 (2), 145-161.

LELEVIER, R., LASHER, G. J. \& BJORKLUND, F. 1955 Effect of a density gradient on Taylor instability. University of California Radiation Laboratory, Report UCRL4459.

LESIEUR, M. 1987 Turbulence in Fluids. Martinus Nijhoff. pp. 90-92.

LESIEUR, M. 1990 Turbulence in Fluids Second Revised Edition. Kluwer Academic Publishers. (Dordrecht) pp. 146-152.

LESLIE, D.C. 1973 Developments in the theory of turbulence. Clarendon Press. 317-325.

LEWIS, D.J. 1950 The instability of liquid surfaces when accelerated in a direction perpendicular to their planes. II Proc. Roy. Soc. Lond. A202, 81-96.

LI, X. L. 1993 Study of three dimensional Rayleigh-Taylor instabilities in compressible fluids through level set method and parallel computation. Phys. Fluids A, 5, 19041913.

LINDEN, P.F., REDONDO, J.M. \& CAULFIELD, C.P. 1992 Molecular mixing in Rayleigh-Taylor instability. Advances in Compressible Turbulent Mixing (ed. Dannevik, W. P. Buckingham A. C. \& Leith, C. E.) National Technical Information Service, U.S. Department of Commece, 95-104.

LINDEN, P.F., REDONDO, J.M. \& YOUNGS, D.L. 1994 Molecular mixing in RayleighTaylor instability. J. Fluid Mech. 265, 97-124.

MARKATOS, N. C. 1986 The mathematical modelling of turbulent flows. Appl. Math. Modelling 10, pp. 190-220.

MESHKOV, E. E. 1969 Instability of the interface of two gases accelerated by a shock wave. Fluid Dyn. 4, 101. [ Izv. Akad. Nauk SSSR, Mekh. Zhidk. Gaza 5, 151 (1969)]

MITCHNER, M. \& LANDSHOFF, R. K. M. 1964 Rayleigh-Taylor instability for compressible fluids. Phys. Fluids. 7(6), 862-866.

MURRAY, J.D. 1965 On the mathematics of fluidization. Part 1. Fundamental equations and wave propagation. J. Fluid Mech. 21 (3), 465-493. 
NAGANO, Y. \& HISHIDA, M. 1987 Improved form of the K- $\varepsilon$ model for wall turbulent shear flows. J. Fluids Engr. 109, 156-160.

NEUVAZHAEV, V. E. 1975 Theory of turbulent mixing. Dokl. Akad. Nauk SSSR 222, 1053-1056.

NEUVAZHAEV, V. E. 1989 Numerical calculation of interfaces turbulent mixing by Rayleigh-Taylor instability on the basis of semiempirical models. International Workshop on Compressible Turbulent Mixing. Pleasanton, CA, Nov. 1989.

NEUVAZHAEV, V. E. \& YAKOVLEV, V. G. 1984 Model and method for numerical calculation of turbulent mixing interfaces moving with acceleration. Problems of Atom. Science \& Engineering (Series: Methods \& Programs for numerical solution of Math. Physics Problems) 2, 17.

NG, K. H. \& SPALDING, D. B. 1972 Turbulence model for boundary layers near walls. Phys. Fluids 15(1), 20-30.

NIKIFOROV, V. V. 1991 Calculation of gravitational turbulent mixing in non-automodel flows. 3rd International Workshop on The Physics Of Compressible Turbulent Mixing. Abbey of Royaumont (France), preprint.

ORSAG, S.A. 1970 Analytical theories of turbulence. J. Fluid Mech. 41(2), 363-386

POLYONOV, A. V. 1989a To the problem of automodel slope determination at gravitational mixing development. International Workshop on Compressible Turbulent Mixing. Pleasanton, CA Nov. 1989.

POLYONOV, A. V. $1989 b$ The heterogeneous K- $\varepsilon$ model of gravitational mixing. International Workshop on Compressible Turbulent Mixing. Pleasanton, CA Nov. 1989.

POPIL, R. \& CURZON, F.L. 1979 Production of reproducible Rayleigh-Taylor instabilities. Rev. Sce. Instr. 50(10), 1291-1295.

RATAFIA, M. 1973 Experimental investigation of Rayleigh-Taylor instability. Phys. Fluids 16(8), 1207-1210.

RAYLEIGH, LORD. 1883 Proc. Lond. Math. Soc. 14, 170-177. (also Scientific Papers, Vol. 2, pp. 200-207)

READ, K.I. \& YOUNGS, D.L. 1983 Experimental investigation of turbulent mixing by Rayleigh-Taylor instability. AWRE Report No. 011/83.

READ, K.I. 1984 Experimental investigation of turbulent mixing by Rayleigh-Taylor instability. Physica D 12, 45-58.

RICHTMYER, R.D. 1954 Taylor instability in shock acceleration of compressible fluids. Los Alamos ScientificLaboratory, Los Alamos ScientificLaboratory Report, LA-1914. 
RICHTMYER, R.D. 1960 Taylor instability in shock acceleration of compressible fluids. Comm. Pure Appl. Math. 13, 297-319.

ROBERTS, A.E. 1945 Stability of a steady plane shock. Los Alamos ScientificLaboratory, Los Alamos ScientificLaboratory Report, LA-299.

ROSE, H. A. \& SULEM, P. L. 1978 Fully developed turbulence and statistical mechanics. Journal de Physique, 39, 441-484.

ROTTA, W. 1951 Statistische Theorie nichthomogener Turbulenz. Z. Physik. 129, $547-572$.

SAFFMAN, P. G. 1970 A model for inhomogeneous turbulence. Proc. Roy. Soc. A 317, 417-433.

SAFFMAN, P. G. \& WILCOX, D. C. 1974 Turbulence-model predictions for turbulent boundary layers. AIAA Journal 102(4), 541-546.

SANDOVAL, D. L. 1995 Dynamics of variable density turbulence. Doctoral Dissertation, University of Washington (Seattle).

SCHUBAUER, G. B. \& TCHEN, C. M. 1959 Turbulent Flows and Heat Transfer, edited by C. C. Lin (Princeton University Press, Princeton, N. J.) Volume 5, page 75.

SHARP, D. H. 1984 An overview of Rayleigh-Taylor instability. Physica D 12, 3-18.

SMEETON, V. S. \& YOUNGS, D. L. 1987 Experimental investigation of turbulent mixing by Rayleigh-Taylor instability. Part 3. AWRE Reprot No. O 35/87.

SNIDER, D. M. \& ANDREWS, M. J. 1994 Rayleigh-Taylor and shear driven mixing with an unstable thermal stratification. Phys. Fluids. (Accepted for publication)

SPALDING, D. B. 1985a Two-fluid models of turbulence. CFD Report No. CFDU/85/4, Imperial College.

SPALDING, D. B. 1985b The numerical computation of multi-phase flows. CFD Report No. CFDU/85/7, Imperial College.

STAFFORD, L. G. 1982 An experimental investigation of turbulent mixing due to buoyancy forces in unstably stratified media. CFDU report No. CFD/82/10.

STEINKAMP, M.J., CLARK, T.T. \& HARLOW, F.H. 1995 Stochastic interpenetration of fluids. Los Alamos National Laboratory, Los Alamos National Laboratory Report. LA-13016-MS

TAKABE, H., MIMA, K., MONTIERTH, L., \& MORSE, R.L. 1985 Self-consistent growth rate of the Rayleigh-Taylor instability in an ablatively accelerating plasma.

Phys. Fluids 28 (12). 3676-3682. 
TAYLOR, G.I. 1950 The instability of liquid surfaces when accelerated in a direction perpendicular to their plane. I. Proc. Roy. Soc. Lond. A201, 192-196.

TRAVIS, J.R., HARLOW, F.H. \& AMSDEN, A.A. 1976 Numerical calculation of twophase flows. Nuclear Science and Engineering 61, 1-10.

TRYGGVASON, G. 1988 Numerical simulations of the Rayleigh-Taylor instability. J. Comp. Phys. 75, 253-282.

TRYGGVASON, G \& UNVERDI, S. O. 1990 Computations of three-dimensional Rayleigh-Taylor instability. Phys. Fluids A 2(5), 656-659.

VON KARMAN, T. \& HOWARTH, L. 1938 On the statistical theory of isotopic turbulence. Proc. Roy. Soc. A 164, 192-215.

WELCH, J. E., HARLOW, F. H., SHANNON, J. P. \& DALY, B. J. 1966 The MAC method. Los Alamos Scientific Laboratory, Los Alamos Scientific Laboratory Report $L A-3425$.

YOUNGS, D.L. 1984 Numerical simulation of turbulent mixing by Rayleigh-Taylor instability. Physica D 12, 32-44.

YOUNGS, D.L. 1989 Modelling turbulent mixing by Rayleigh-Taylor instability. Physica D 37, 270-287.

YOUNGS, D.L. 1991 Three-dimensional numerical simulation of turbulent mixing by Rayleigh-Taylor instability. Phys. Fluids A 3(5), 1312-1320.

YOUNGS, D.L. 1992a Experimental investigation of turbulent mixing by Rayleigh-Taylor instability. Advances in Compressible Turbulent Mixing (ed. Dannevik, W. P. Buckingham A. C. \& Leith, C. E.) National Technical Information Service, U.S. Department of Commece, 607-626.

YOUNGS, D.L. 1992b A two-dimensional turbulence model based on the equations of multiphase flow. Proceedings of the Third Zababakhin Scientific Talks. Kyshtym, USSR, Jan. 14-17.

YOUNGS, D.L. 1994 Numerical simulation of mixing by Rayleigh-Taylor and RichtmyerMeshkov instabilities. Laser and Particle Beams 12(4), 00-00. (preprint). 


\section{Acknowledgments}

The list of people who have helped me through this endeavor is long. Foremost, I would like to express my appreciation to my academic advisor, Prof. Roy A. Axford at the University of Illinois, and to my mentor here at Los Alamos National Laboratory (LANL), Dr. Francis H. Harlow, Laboratory Fellow.

Two T-3 staff members that have been extremely helpful are Dr. Rick Rauenzahn and Dr. Timothy Clark. Tim and I have had numerous helpful discussions on this topic and related topics over the past couple of years. Rick, whom I am very indebted to, has been extremely generous with both his time and insightful suggestions concerning problems we have encountered along the way. His help and collaboration have proved invaluable to this work.

I would also like to extend my gratitude to Group T-3, Fluid Dynamics in the Theoretical Division at LANL. My appreciation extends to Dr. John Hopson (T-3 group leader) and T. Daniel Butler (T-3 deputy group leader) for providing me with funding while I was in residence as a graduate student at LANL. Also to the group secretary, Margaret Findley, who has helped in the preparation of this manuscript. T-3 staff members such as Dr. Mathew Maltrud, and Dr. Douglas Kothe have provided much appreciated friendships and intellectual guidance along the way. Other graduate students in T-3 that deserve mention, include Eric Harsted, Rodman Linn, Chris Zusi, Don Sandoval, and Casildo Romero.

A special mention must also go to my committee members at the University of Illinois, the list includes Prof. Ronald Adrian, Prof. Thomas Hanratty, and Prof. Daniel Riahi. 


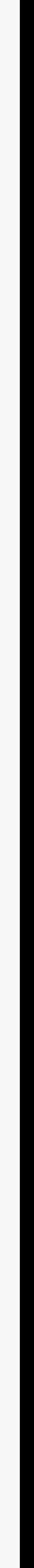


This report has been reproduced directly from the best available copy.

It is available to DOE and DOE contractors from the Office of Scientific and Technical Information, P.O. Box 62,

Oak Ridge, TN 37831.

Prices are available from

(615) 576-8401.

It is available to the public from the

National Technical Information Service,

US Department of Commerce,

5285 Port Royal Rd.

Springfield, VA 22616. 


\section{Los Alamos \\ NATIONAL LABORATORY \\ Los Alamos, New Mexico 87545}

